\title{
Chromatin State Dynamics Confers Specific Therapeutic Strategies in Enhancer Subtypes of Colorectal Cancer
}

Elias Orouji ${ }^{1,7, \#}$, Ayush T. Raman 1,2,8,\#, Anand K. Singh ${ }^{1, \#}$, Alexey Sorokin ${ }^{3}$, Emre Arslan ${ }^{1}$, Archit K. Ghosh ${ }^{1}$, Jonathan Schulz ${ }^{1}$, Christopher J. Terranova ${ }^{1}$, Ming Tang ${ }^{1}$, Mayinuer Maitituoheti ${ }^{1}$, S. Carson Callahan ${ }^{1}$, Katarzyna J. Tomczak ${ }^{1}$, Zhiqin Jiang ${ }^{3}$, Jennifer S. Davis ${ }^{9}$, Sukhen Ghosh ${ }^{6}$, Hey Min Lee ${ }^{3}$, Laura Reyes-Uribe ${ }^{5}$, Kyle Chang ${ }^{5}$, Yushua Liu ${ }^{4}$, Huiqin Chen ${ }^{4}$, Ali Azhdarnia ${ }^{6}$, Jeffrey S. Morris ${ }^{4,10}$, Eduardo Vilar ${ }^{5}$, Kendra S. Carmon ${ }^{6}$, Scott Kopetz $^{3}$ and Kunal Rai ${ }^{1, *}$

1 Department of Genomic Medicine, The University of Texas MD Anderson Cancer Center, Houston, TX, USA

${ }^{2}$ Graduate Program in Quantitative and Computational Biosciences, Baylor College of Medicine, Houston, TX, USA

${ }^{3}$ Department of Gastrointestinal Medical Oncology, The University of Texas MD Anderson Cancer center, Houston, TX, USA

${ }^{4}$ Department of Bioinformatics and Biostatistics, The University of Texas MD Anderson Cancer Center, Houston, TX, USA

${ }^{5}$ Department of Clinical Cancer Prevention, The University of Texas MD Anderson Cancer Center, Houston, TX, USA

${ }^{6}$ Center for Translational Cancer Research, University of Texas Health Science Center at Houston, Houston, TX, USA

${ }^{7}$ Present address: Epigenetics Initiative, Princess Margaret Genomics Centre, Toronto, ON, Canada

${ }^{8}$ Present address: Broad Institute of MIT and Harvard, Cambridge, MA, USA

${ }^{9}$ Department of Epidemiology, The University of Texas MD Anderson Cancer Center, Houston, TX, USA

${ }^{10}$ Present address: Department of Biostatistics, Epidemiology and Informatics, Perelman School of Medicine, University of Pennsylvania, Philadelphia, PA.

\# These authors contributed equally.

${ }^{*}$ Correspondence should be addressed to KR (krai@mdanderson.org) 


\begin{abstract}
The extent and function of chromatin state aberrations during colorectal cancer (CRC) progression is not completely understood. Here, by comprehensive epigenomic characterization of 56 tumors, adenomas, and their matched normal tissues, we define the dynamics of chromatin states during the progression of colorectal cancer. H3K27ac-marked active enhancer state could distinguish between different stages of CRC progression. By epigenomic editing, we present evidence that gains of tumor-specific enhancers for crucial oncogenes, such as ASCL2 and FZD10, was crucial for excessive proliferation. Consistently, combination of MEK plus bromodomain (BET) inhibition was found to have synergistic effects in CRC patient-derived xenograft (PDX) models. Probing inter-tumor heterogeneity, we identified four distinct enhancer subtypes (EpiC), three of which correlate well with previously defined transcriptomic subtypes (CMSs). Importantly, CMS2 can be divided into two EpiC subgroups with significant survival differences. Leveraging such correlation, we devised a combinatorial therapeutic strategy of enhancer-blocking bromodomain inhibitors with pathway-specific inhibitors (PARPi, EGFRi, and TGF $\beta$ i) for three EPIC groups. Our data suggest that the dynamics of active enhancer underlies colorectal cancer progression and the patient-specific active enhancer patterns govern their unique gene expression patterns which can be leveraged for precision combination therapy.
\end{abstract}




\section{INTRODUCTION}

Colorectal cancer (CRC) is the third most common cancer in the world. Most cases of $\mathrm{CRC}$ originate from premalignant lesions, mainly adenomas, which can progress into malignant adenocarcinomas(1). Genomic alterations in colorectal adenocarcinomas frequently occur in $A P C$ (83\%), TP53 (60\%), KRAS (43\%), and BRAF (10\%) genes(2-6). However, targeted therapy for these mutational subgroups is not well established. This calls for a comprehensive approach to identify unique signatures derived from various molecular characteristics of tumors including genomic and epigenomic components which, along with clinical features, can be used to introduce clinically feasible targeted therapeutic strategies.

One such effort toward precision therapy was led by the CRC subtyping consortium, which identified four consensus molecular subtypes (CMSs) with distinct activation patterns of specific oncogenic signaling pathways using gene expression datasets(3). Briefly, these transcriptomic subgroups include the immune subtype (CMS1), canonical subtype (CMS2), metabolic subtype (CMS3), and mesenchymal subtype (CMS4)(7). Although distal cis-regulatory elements are known to play a central role in controlling transcription patterns, the bona fide contributions of these elements in defining CMSs are not understood. Importantly, epigenetic processes are reversible and can provide actionable targets for therapeutic development, such as bromodomain inhibitors (BRDi) that target super-enhancer elements(8). The benefits of targeting the CRC epigenome have already been demonstrated by associations of the CpG island methylator phenotype (CIMP) with BRAF mutations, for which efforts are underway to devise combinatorial strategies targeting methylation and the BRAF oncogene(3). Consistent with this finding, we and others have provided evidence that epigenomic alterations at the level of DNA methylation play crucial roles in CRC initiation(9) and oncogenic function(10).

The current literature on alterations of enhancer elements in CRC is scarce, with only a handful of studies(11-13) with small sample sizes that profiled primary tumors. Furthermore, a comprehensive understanding of other chromatin state alterations and their functional contribution to tumor progression is mostly lacking. Therefore, it is of immense importance to define the relationship between chromatin aberrations and genomic and transcriptomic features in tumors. 


\section{RESULTS}

To characterize the epigenomic makeup of CRC, we first generated ChIP-seq (chromatin immunoprecipitation followed by massive parallel sequencing)-based profiles for six histone marks including H3K4me1 and H3K27ac, associated with enhancer regions; H3K4me3, associated with promoter regions; H3K79me2, associated with transcribed regions; H3K9me3, associated with heterochromatin regions; and $\mathrm{H} 3 \mathrm{~K} 27 \mathrm{me} 3$, associated with Polycomb repression in a total of $16 \mathrm{CRCs}$ and 17 matching adjacent normal colonic mucosa samples (Table S1). With the use of the ChromHMM algorithm(14), we trained a 10-state, 12-state, and 15-state model (for samples having all the six marks; Table S1) consisting of combinatorial patterns of specific histone modifications known as chromatin states, which are linked with the transcriptional output of the associated genomic loci and regulate the recruitment of various components of the transcriptional machinery. We chose a 10-state model for in-depth investigation because it represented biologically interpretable combinatorial patterns that were not repetitive or ambiguous (Fig. 1A and S1A). We annotated these chromatin states based on the content of each state and its average relative distribution around known genomic features (Fig. S1B). To define the chromatin states that best differentiate between normal and tumors, we developed a statistical approach, termed ChromXplorer (see Methods; Fig. S1C), that utilizes the ChromDiff(15) framework for all genomic loci instead of a gene-centric approach. The ChromXplorer-based dimensionality reduction approach showed the "active enhancer" state, but not others, to be the most discriminatory state in all three investigated models (Fig. 1B and Fig. S1D-S1F). Analysis of singular histone marks indicated that the active enhancer mark, defined by H3K27ac, also led to clustering of the samples according to their phenotype (Fig. S2A), whereas some of the other histone marks such as H3K79me2, H3K9me3, and H3K27me3 showed weaker clustering patterns due to variations among tumor samples (Fig. S2A), suggesting de novo gain or loss of H3K27ac patterns as an important distinguishing feature of CRC.

In light of this, we expanded the cohort by profiling the H3K27ac mark in additional colorectal tumors $(n=17)$, adenomas $(n=4)$, normal crypts $(n=4)$, and cancer cell lines $(n=11)$ to characterize the enhancer patterns through the various stages of progression. Interestingly, distinct enhancer patterns appeared to define each progression stage, suggesting that these enhancer patterns may evolve as the disease progresses (Fig. 1C). Furthermore, the established CRC cell lines formed a separate cluster with three cell lines that partially overlapped with colorectal adenocarcinoma tumors (Fig. 1C). Similarly, computation of correlation matrix identified a higher similarity within normal samples as opposed to tumor samples which segregated into two 
subgroups, indicative of greater heterogeneity between adenocarcinoma specimens (Fig. S2B).

To identify recurrent cancer-specific peaks, shared H3K27ac peaks in all tumor samples were summed up and overlapped with shared normal or shared adenoma enhancers. Our analysis identified 32,176 tumor-specific $(T)$ enhancers, 12,822 normal-specific $(N)$ enhancers, and 4,998 adenoma-specific (A) enhancers (Fig. 1D). Notably, 5,759 enhancers were present in both adenomas and adenocarcinomas, but not in normal tissue, whereas a much higher number $(32,176)$ were specific to adenocarcinomas only, thereby, suggesting that several enhancers are gained in de novo manner in the adenocarcinoma stage. Furthermore, the average intensity of enhancer peaks was found to progressively increase in adenomas and colorectal tumors (Fig. S2C). Similar to typical enhancers, we found super-enhancer elements (SE), that are thought to promote oncogene expression, to be upregulated in adenocarcinomas (Fig. 1E). We identified 886 adenocarcinoma-specific, 93 adenoma-specific, and 193 shared super-enhancers between adenomas and adenocarcinomas using ROSE algorithm(16) (Fig. 1E).

Active enhancers specific to each disease progression stage marked important driver genes that were identified based on the presence of enhancers in their proximal regulatory regions as previously defined(17,18) (Fig. 1F and Table S2). For example, regulators and mediators of the cell cycle, DNA damage, stress and cytokine and metabolism harbored gains in a tumor-specific manner. Conversely, enhancers for regulators of T-cell differentiation, MHC class II antigen presentation, vitamin A metabolism, chemokine signaling, and focal adhesion, among others, were specifically present only in normal tissues and were lost during progression. Enhancers common to premalignant adenomas and tumors were enriched around regulators of cell fate, collagen degradation, metabolic transport, and WNT signaling (Fig. 1F). We noted that the enrichment of enhancers/super-enhancers in the proximity of key WNT signaling regulators such as ASCL2, SLL4, FZD10, CTNNB1 ( $\beta$-catenin), AXIN2, TCF7L1 (TCF3), TCF7L2 (TCF4), and a subset of these correlated with their progressive increase in gene expression (Fig. 1G-H, Fig. S3A-C, and Table S2). This suggests that the genes identified to harbor active enhancers or super-enhancers near their vicinity or in regions interacting with the gene body/promoters are likely to contribute to the carcinogenic properties. Enhancers are epigenetic platforms for binding of various transcription factors (TFs), and motif enrichment analysis has suggested that specific classes of TFs such as forkhead (FOXA1, FOXA2, and FOXM1), ETS (ETV4, ERG, and FLI1), and bZIP (ATF3 and AP-1) are overrepresented in the tumor-specific active enhancers (Fig. 1I and Fig. S3D). Importantly, homeobox (NKX3.1), IRF (IRF1, IRF2), bHLH (NeuroG2, TCF4), and 
FOXO1 motifs were enriched in active enhancers that were lost during tumor progression (Fig. 1I and Fig. S3D).

\section{Inactivation of tumor-specific enhancers leads to the transcriptional and phenotypical switch}

To test the functional role of some of the specifically activated tumor-specific enhancers, we first ranked the top differentially activated enhancers (Fig. 2A and Table S3; see Methods). We chose to functionally test some of the top enhancers from this list that were located in the vicinity of two WNT signaling genes: ASCL2 and FZD10. ASCL2 is a known driver of intestinal stem cell phenotype(19) and a predicted oncogene in $\operatorname{CRCs}(20)$ whereas, $F Z D 10$ is a transmembrane protein that acts as a WNT receptor driving downstream signaling, which is a key signaling event in CRCs(7). Moreover, established CRC cell lines harbored a significant percent of tumor-specific H3K27ac peaks (Fig. S4A). We used dCas9-KRAB and specific gRNAs to suppress the activity of target enhancer loci in SW480 cells, a well-characterized model with mutations in $A P C, K R A S$, and $T P 53^{20}$. For ASCL2, we chose to focus on a distal super-enhancer that was present in the adenomas and adenocarcinomas, but not normal tissues (Fig. 2B, $\mathbf{1 H}$, and S3A). HiChIP and $3 \mathrm{C}$ experiments confirmed the direct interaction of this super-enhancer with the ASCL2 gene promoter (Fig. 2B and S4B). We noted that all three gRNAs targeting this distal super-enhancer region were able to suppress the H3K27ac signal in their respective target regions (Fig. 2C). These gRNAs also downregulated gene expression (Fig. 2D) and downstream proliferation of SW480 cells, suggesting that the distal super-enhancer acts as a functional driver event in this cell line (Fig. 2E). For the FZD10 locus, we noted that gRNAs targeting E1, E5, and E6 regions reduced the $\mathrm{H} 3 \mathrm{~K} 27 \mathrm{ac}$ signal which was consistent with the observations from HiChIP experiments showing the interaction of these enhancers with the promoter of this gene (Fig. S4C4D). Epigenetic suppression of these enhancers also downregulated gene expression and decreased cell proliferation, suggesting multi-enhancer regulation of FZD10 transcription (Fig. S4E-4F) and the contribution of this locus to the proliferative properties of CRC cells (Fig. S4F).

Although these studies provide evidence for the functionality of individual enhancers, a holistic approach would be to block many such enhancers using BRDi, which are being actively tested in clinical trials in solid tumors. Since ASCL2 and WNT pathways have established roles in regulating stem cell function in both normal and cancer tissues $(7,19,20)$, we asked whether targeting enhancer-blocking agents to stem cells, using the LGR5 antibody, would result in suppressing the growth of the cells. Hence, we synthesized an antibody-drug conjugate (ADC) 
by using LGR5 antibody and the RVX208 which is a BRDi in phase 3 trials for cardiovascular indications(21). Indeed, LGR5-RVX208 (Fig. S5A) ADCs specifically blocked the growth of SW480 cells or patient-derived human colon organoids in an LGR5 expression-dependent fashion (Fig. 2F-2G and Fig. S5B-E). These studies established that some of the activated enhancers in tumors may play functional roles in imparting pro-tumorigenic properties to CRC.

Treatment of four different patient-derived xenograft (PDX) models (C1138, F3053, F3008, and B8131) with BRDi (iBET-151) caused strong tumor reduction in one model but showed no significant difference in 3 of 4 models, suggesting a context-dependent impact of enhancer blockade (Fig. 2H-I). Since chromatin aberrations are believed to be a permissive event for activation of pro-tumorigenic transcriptional signature upon oncogenic signaling, we reasoned that blockade of enhancers may be more effective in combination with inhibition of key oncogenic driver signaling pathways. We noted that a number of upstream regulators of the MEK pathway, including EGFR, NRAS, KRAS, FGFR3, MAPK3K1, MAPK11, MAPK12, MAP3K20, MAP3K10, AKT2, IRS2, FGF1, FGF3, FGF19, and FGF21, gained enhancers in colorectal tumors in comparison to adjacent normal tissues or adenomas (Fig. 2J and S6A). Therefore, we analyzed whether enhancer-blocking BRDi may synergize with MEKi, which are actively being used in CRC clinical trials, to enhance therapeutic efficacy. Indeed, the treatment of four different PDX models (C1138, F3053, F3008, and B8131) showed significant synergy between MEKi (trametinib or binimetinib) and BRDi in 3 of these 4 models (Fig. 2H-I and S6B). Although monotherapy with MEKi showed moderate activity on tumor growth in all of the models, significant tumor regression was noted in B8131, and two models (F3052 and 3008) showed slower growth in combinatorial treatment with BRDi. These data highlighted the differential responses of various PDX models with BRDi and suggested that BRDi plus MEKi could be a successful therapeutic strategy in a subset of CRC patients.

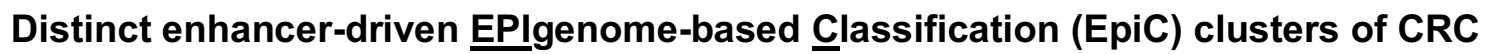

As exemplified by the MEKi + BRDi combination treatment results, all CRC tumors do not respond to the blanket therapies equally. This motivated us to perform molecular characterization of CRC tumors based on their epigenomic content, which can be potentially useful for patient stratification and subsequent assignment of each subtype to particular combination treatment with epigenetic compounds.

We classified adenomas and adenocarcinomas by using a consensus non-negative matrix 
factorization (NMF)-based clustering method that uses the enriched H3K27ac peaks. Based on the cophenetic and dispersion scores, we identified four distinct clusters of tumors (Fig. 3A, S7A S7B, and Table S4), hereafter referred to as EPIgenome-based Classification (EpiC) clusters: EpiC1, EpiC2, EpiC3, and EpiC4. The average intensity of enhancer peaks within these clusters varied such that EpiC2 and EpiC4 enhancers had significantly higher peaks than the other two clusters had (Fig. S7C-S7D). EpiC3 clusters had the least overall peak intensity (Fig. S7C-S7D). However, differential regions across these subtypes were found to be specific to particular genes (Fig. S8A).

Previously, four CMSs had been defined on the basis of transcriptomic signatures of more than four thousand CRC samples (3) as the CMS1 (MSI immune subtype), CMS2 (canonical subtype), CMS3 (metabolic subtype), and CMS4 (mesenchymal subtype). We classified the tumors used in our study into these CMSs, for which we had gene expression data, by using the CMS classifier(22), and based on the corresponding trained model(3), we computed their EpiC subtype. Interestingly, we determined that epigenomic clusters (EpiCs) significantly overlap their assigned CMSs (Fig. 3A). All of the samples classified as CMS1 (immune) subtype overlapped with the EpiC1 subtype. Although only two patients had been assigned to the CMS4 (mesenchymal) subtype, strikingly, all four adenoma samples along with these two patients clustered as the EpiC2 subtype. It has been shown that adenoma lesions have mesenchymal properties and may progress to adenocarcinoma due to aberrations in the Ras signaling pathway (23). Moreover, consensus NMF clustering divided the canonical subtype or CMS2 into two novel subsets of epigenomic CRC clusters, EpiC3 and EpiC4 (Fig. 3A). Of note, the metabolic subtype or CMS3 is not detectable with the use of enhancer-based epigenomic clustering and is scattered in multiple subtypes.

Interestingly, pathway analysis based on the gene targets of enhancers (see Methods) that are specifically enriched in each EpiC subtype showed similarity to those known to be CMSspecific (3) (Fig. 3B). Enhancers specific to EpiC1, which overlaps significantly with CMS1, were in the proximity of genes regulating immune response, B- and T-cell activation, and inflammation. EpiC2, which overlaps with CMS4 and interestingly contains all adenoma samples, regulates the TGF $\beta$, WNT, and VEGF pathways (Fig. 3B). CMS2 CRC tumors were distributed into two epigenetic subtypes EpiC3 and EpiC4. EpiC3 enhancers enriched on targets of c-MYC, EGFR, MAPK, and TAp63 signaling, whereas EpiC4 enhancers activated genes involved in GPCR, IGF, PI3K-AKT and neurotransmitter signaling (Fig. 3B and Table S5). Examples of genes near the 
enhancers in each EpiC category are shown in Fig. 3C and Fig. S8.

We also noted that EpiC3 enhancers were more spread (or "broad") in nature whereas EpiC4 enhancers were narrower in their width (or "Sharp") (Fig. 3D). Systematic analysis of broad $(>5 \mathrm{~kb})$ and sharp (<3kb) peaks (see Methods) showed enrichment of broad peaks in EpiC3 versus sharp peaks in EpiC4 enhancers (Fig. 3D and S7E).

To determine if the observed dichotomy of CMS2 tumors into EpiC3 and EpiC4 in our cohort expanded to a larger set of CRC samples, we examined the clustering of CMS2-designated TCGA tumors (24) based on the gene targets of EpiC3 and EpiC4 enhancers. NMF clustering of 115 CMS2 tumors (see Methods) identified 3 clusters (Fig. S9A and Table S6). Overlap of EpiC4 enhancer target genes with Cluster2-specific genes showed enrichment of EpiC4-specific pathways such as GPCR, PI3K pathway and NCAM mediated neurotransmitter signaling (including NPTX2 - a differentially expressed gene between EpiC3 and EpiC4) (Fig. S9B-D). Overlap of EpiC3 enhancer target genes with Cluster3-specific genes showed enrichment of EpiC3-specific pathways such as EGFR signaling, MAPK, and c-MYC targets (Fig. 3E and S9BC). On the contrary, EpiC3-target genes overlap with Cluster3-specific genes or EpiC4-target genes overlap with Cluster2-specific genes did not enrich in any pathways specific for EpiC3 or EpiC4 (Fig. S9C). Cluster 1 specific genes were mostly enriched in immune pathways suggesting likely separation due to low tumor content (Fig. S9C). Therefore, we designated Cluster2 $(n=37)$ as 'EpiC4-like' and Cluster3 $(\mathrm{n}=63)$ as 'EpiC3-like'. Importantly, EpiC3-like tumors showed poorer survival in comparison to EpiC4-like tumors (Fig. 3F), thus providing clinical significance for enhancer-based classification of CRC tumors.

To identify potential regulators of the EpiC-specific features, we performed motif analyses of enhancers specific to each EpiC subtype using HOMER (25). Interestingly, distinct TF classes were enriched in each of the EpiC subtypes. bZIP (BATF) and ZF (GATA6) type TFs were enriched in EpiC1, and homeobox (BRN2 or POU3F2) and Stats (STAT4) were enriched in EpiC2; c-MYC (bHLH) was enriched in EpiC3, and HMGs (SOX9) were enriched in EpiC4 (Fig. 3G). Consistently, we noted that expression of TFs (except for POU3F2) was higher, specifically in the respective CMSs in TCGA samples that corresponded with EpiC subtypes (Fig. S9E). Importantly, previous studies have implicated c-MYC(26-29), GATA6(30-32), SOX9(33-35), and STAT4(36,37) in CRC progression. BRN2/POU3F2 has oncogenic roles in melanoma(38,39), and BATF2, a BATF family member, has been implicated in $\operatorname{CRC}(40)$. These data suggest that 
the potential origin of different EpiC subtypes may be due to the pioneering activity of TFs belonging to different classes of TF families.

\section{Combinatorial treatments for newly identified EpiCs}

The CMS classification is being tested as a prognostic indicator for use in targeted therapy in CRC. For example, the CMS1 and CMS4 subtype cell lines are more responsive to HSP90 inhibitor, whereas the CMS2 subtype shows more sensitivity to WNTi and EGFRi(41,42). The CMS3 subtype is enriched in metabolic pathways and RAS mutations, but therapeutic vulnerabilities are unknown(3).

Exploring the clinical implications of our findings, we used a panel of 11 signaling pathway inhibitors that are known to be involved in the CRC tumorigenesis and whose key regulators showed differential enhancer enrichments in their vicinity in different epigenomic subtypes of CRC. Therefore, we treated six CRC cell lines with the signaling pathway compounds along with a panel of 8 known epigenetic inhibitors to treat cell lines from different CMSs, previously annotated with use of a CMS classifier (Fig. S10A, S10B, S11, S12, S13A, and S13B) $(41,42$ ). We further analyzed both $\mathrm{IC}_{50}$ and $\mathrm{AUC}$ indices to choose compounds with the best potency as well as the highest efficiency (Fig. 4A-4C, S10B, S13A). We used a combination of one signaling pathway inhibitor along with the most efficient epigenetic inhibitor according to the generated drug matrix in distinct CMSs. Based on the analysis of monotherapies, we tested combinations of BRDi + PARPi for CMS1/EpiC1, BRDi + EGFRi for CMS2/EpiC3-4, and BRDi + TGF $\beta$ i for CMS4/EpiC2 and found the combinations to perform better in reducing cell growth in vitro (Fig. 4D-4F and Figs. S13B and S14A). Importantly, we observed drastically enhanced activities of these combinations in the in vivo experiments carried out in nude mice, compared with the results of experiments using monotherapies (Fig. 4G-4I).

Importantly, these combinatorial therapies demonstrated specific activity to the corresponding CMS/EpiC and had a minor impact on other CMS/EpiC subtypes. Specifically, BRDi (iBET-151, 15mg/kg) + PARPi (Olaparib, 50mg/kg) showed more significant impact on CMS1/EpiC1 cells than CMS2/EpiC3-4 or CMS4/EpiC2 did; similarly, BRDi (iBET-151, 15mg/kg) + EGFRi (Gefitinib, 100mg/kg) impacted CMS2/EpiC3-4 cells specifically, and BRDi (iBET-151, $15 \mathrm{mg} / \mathrm{kg})+\mathrm{TGF} \beta \mathrm{i}(\mathrm{SB} 431542,10 \mathrm{mg} / \mathrm{kg})$ reduced tumor growth of CMS4/EpiC2 cells specifically (Fig. 4G-4I). Notably, we did not observe much loss in tumor weight upon treatment with these drug combinations suggesting little or no side effects of these therapies (Fig. S14B). 


\section{DISCUSSION}

Our study provides the most comprehensive data on basic epigenetic elements, in line with definitions from Roadmap/ENCODE, for human CRC, pre-malignant adenomas, widely used cell lines, and normal crypts/tissues. Importantly, these tissues/cell lines have associated genomic, transcriptomic, drug-response, and clinical datasets available. Thus, these collective epigenomic maps elucidate the epigenomic makeup of CRC and are an essential resource, along with the previous datasets(11-13), for the community to generate hypotheses regarding the roles of the epigenome in CRC progression. Based on this comprehensive resource, our unbiased chromatin state analyses identified the H3K27ac marked active enhancers are the most discriminatory epigenetic elements between the various histopathological stages of CRCs. Consistent with this, the number of enhancer peaks that differentiate adenomas, normal tissues, and tumors is very high, suggesting that enhancer activation is likely a major epigenetic aberration in $\mathrm{CRC}$. We suggest that enhancer aberrations are a critical component of a permissive chromatin environment in cancer cells that allow oncogenic events/pathways to drive excessive tumor growth. In addition to the active enhancer state, we also noted the heterochromatin state (H3K9me3) to differentiate between tumors and normal tissues. This will need to be further examined for causative effects.

Most importantly, our work identifies heterogeneity in enhancer patterns between CRC patients and for the first time identifies epigenomic subtypes of CRC. We demonstrate that the EpiC subtypes correlate highly with the expression phenotype (CMS), in line with previous observations made in the pan-cancer TCGA ATAC-seq study(43), except that the CMS2 subtype was bifurcated into two distinct EpiC subtypes, EpiC3 and EpiC4. This is an interesting observation since we hypothesize that EpiC3 is likely driven by a c-MYC-regulated transcriptional program, whereas EpiC4 is driven by SOX9 and had exclusive activation of IGF and PI3K-AKT signaling. In addition, only EpiC4 showed activation of enhancers around important neuronal genes (Figure 3C and S8), such as NPTX2(44), which could be either due to neural invasion in this category of tumors(45) or epigenetic derepression of neuronal features. We suggest that inhibitors that target the c-MYC program could be more useful in EpiC3 subtypes, whereas SOX9 may be a target in EpiC4 subtypes. Importantly, the two TCGA subsets with EpiC3 and EpiC4 like features showed significant difference in survival, hence providing a clinical significance of this bifurcation of the CMS2 subtype. How these EpiC-specific enhancer patterns are set-up and how they cooperate with genetic events for the transformation of normal cells into cancers with unique transcriptional signatures need to be studied in the future. 
We identified superenhancers surrounding ASCL2 and FZD10 to be necessary for colorectal cancer cell growth in vitro (Figures 2 and S4). This is interesting in that it provides evidence for enhancer/superenhancer elements as being targets for therapeutics in the future, potentially with the evolution of CRISPR-based therapies. It is also imaginable that eRNAs made from such superenhancers may be targets for RNA-targeted therapeutics such as antisense oligonucleotides (ASOs)(46). The advances in 3D epigenome studies, such as our HiChIP data, also suggest that one enhancer may target many genes and hence could prove to be a better therapeutic target than the presumed gene target itself. We found over $>30,000$ such enhancers to be uniquely activated in colorectal adenocarcinomas. Future studies will be needed to identify functional enhancers such as those surrounding ASCL2 and FZD10. Finally, our data suggest that combination of bromodomain inhibitors as an umbrella approach for blockade permissive enhancer states along with specific driver signaling pathway inhibitors could be used in defined patient CRC populations, hence providing a paradigm for epigenome-focused personalized therapeutic strategy. This is an important observation as it suggests patient-stratification based on enhancer signatures as well as the use of epigenetic therapy in a patient-centric manner. 


\section{METHODS}

\section{Cell culture}

Colorectal cancer cell lines were obtained from the MDACC cell bank. CRC cell lines including SW480, SW837, SW620, SW403, CW2, HCT15, HCT116, DLD1, SNUC5, CW2, and RKO were maintained in L-15 media with $10 \%$ FBS in tissue-culture conditions without $\mathrm{CO}_{2}$.

\section{CRC organoid culture}

Tumor tissue dissociation was performed by using the MACS dissociator (Milteny Biotech). Sterile 24-well plates were pre-warmed in the $37^{\circ} \mathrm{C}$ incubator for $\sim 3$ hours. Matrigel (Corning, Cat\# 356231) was thawed on ice before use. Cells were resuspended in Matrigel at a concentration of $15-25 \mathrm{k}$ cells per $30 \mu \mathrm{l}$ in each well. Matrigel-cell suspension was pipetted to the center of each well, and after 1-2 min at room temperature, the plate was transferred to a $37^{\circ} \mathrm{C}, 5 \% \mathrm{CO}_{2}$ incubator for 5-10 min, followed by the addition of $500 \mu \mathrm{l}$ of complete growth medium. The medium was replaced every 2-3 days and passaged every 7 days. The base medium was Advanced DMEM/F12 (Life Technologies, Gibco) supplemented with B27, 2 mM (Life Technologies, Cat\# 17504044), GlutaMAX-1 (Life Technologies, Gibco), 10 mM HEPES (Life Technologies, Gibco), 1.25 mM N-acetyl cysteine (Sigma-Aldrich, Cat\# A9165), 10 nM [Leu15]-Gastrin 1 (Sigma-Aldrich), and Pen/Strep (Life Technologies, Gibco). Complete growth medium consisted of base medium plus $50 \mathrm{ng} / \mathrm{ml}$ EGF (Life Technologies, Cat\# PMG8043), 100 ng/ml Noggin, $0.5 \mu \mathrm{M}$ A 83-01 (Tocris Bioscience), $10 \mu \mathrm{M}$ Y-27632 (Sigma-Aldrich), 40\% Wnt-3a, 10\% R-spondin 1, $10 \mu \mathrm{M}$ SB202190 (Sigma-Aldrich), and $5 \mu$ l of CHIR 99021 (Tocris Bioscience).

\section{Tissue samples and tissue preparation}

All adenocarcinoma and normal tissue samples were obtained from the Division of Gastrointestinal Medical Oncology patients' databank as frozen samples and were processed according to our modified protocol(47). Crypts were isolated from endoscopic biopsies of Familial Adenomatous Polyposis (FAP) patients that provided written informed consent to an IRBapproved protocol (MD Anderson Cancer Center \#PA12-0327) following previously reported protocol (48). Detailed clinical and pathology characteristics of all samples are provided in Table $\mathrm{S} 1$. Crosslinked tissue samples were stored in a freezer at $-80^{\circ} \mathrm{C}$ before ChIP-seq experiments. 


\section{Chromatin immunoprecipitation followed by sequencing (ChIP-seq)}

Chromatin immunoprecipitation followed by deep sequencing was performed as described previously(49). Chromatin shearing conditions were optimized for colon cells. The following antibodies were used in this procedure; H3K4me1 (ab8895), H3K27ac (ab4729), H3K4me3 (ab8580), H3K79me2 (ab3594), and H3K27me3 (ab6002). Generated ChIP-seq data underwent quality-control measures and was further processed by pyflow-ChIPseq, a snakemake-based ChIPseq pipeline. Briefly, raw reads were mapped to human reference genome hg19 by using bowtie1, and duplicated reads were removed, retaining only uniquely mapped reads. RPKMnormalized bigwigs were generated by deep tools, and the tracks were visualized with the use of an Integrative Genomics Viewer (IGV).

\section{ChIP-seq analyses}

Raw fastq reads for all ChIP-seq experiments were processed by using a snakemake-based pipeline (https://zenodo.org/record/819971). The raw reads were first processed by using FastQC(50), and uniquely mapped reads were aligned to the hg19 reference genome by using Bowtie(51) Ver. 1.1.2. Duplicate reads were removed by using SAMBLASTER(52) before compression to bam files. To directly compare ChIP-seq samples, uniquely mapped reads for each mark were downsampled per condition to 15 million, sorted, and indexed with the use of samtools Ver. 1.5(53). To visualize ChIP-seq libraries on the IGV genome browser, we used deepTools Version 2a.4.0(54), which generated bigWig files by scaling the bam files to reads per kilobase per million (RPKM).

Super ChIP-seq tracks were generated by merging bam files from each phenotype, sorting and indexing by using samtools, and scaling to RPKM by using deepTools. We used the Modelbased analysis of ChIP-seq (MACS) version 1.4.2(55) peak calling algorithm to identify peaks for the sharp marks H3K4me1, H3K27ac, and H3K4me3; we used MACS version 2.1.0 to identify peaks for broad marks H3K9me3, H3K79me2, and H3K27me3. The peak enrichment was calculated over a whole genome "input" background with a p-value of $<1 e-5$. Super-enhancers were identified by using the ranking of the super-enhancers (ROSE) algorithm based on the H3K27ac ChIP-seq data(16). 


\section{ChromHMM analysis}

ChromHMM(14) is used to identify combinatorial chromatin state patterns based on the histone modifications studied. Normalized bam files were converted to bed files and binarized at a 1000bp resolution by using the BinarizeBed command. We programmed ChromHMM to Learn 10 (Fig. 1A), 12 (Fig. S1A, left panel), and 15 (Fig. S1A, right panel) chromatin state models and chose the 10-state model for in-depth characterization and presentation purposes. We chose this model because a lot of the states were replicated in the higher state models, and the 10-state model was large enough to identify important functional elements yet small enough to allow easy interpretation of the biological states. Any marks with an emission probability of $<0.1$ (or $10 \%$ ) were not considered for the annotation of the states.

To determine chromatin state differences between various groups, we used a two-step process using ChromXploreR (Fig. S1C). First, using the segmentation calls from the ChromHMM output, the entire genome was divided into non-overlapping bins of $1000 \mathrm{bp}$. We next counted the number of times a chromatin state was observed in each of the bins and obtained a frequency matrix for each state across all of the samples in the ChromHMM model (E1-E10). For visualization purposes, the frequency matrix was binarized such that if a bin contained a non-zero value for a sample, it was counted as 1 , otherwise as 0 . The top 10,000 most variable bins were used for further analysis. Most variable bins or regions were computed with the use of the FSbyVar function from the CancerSubtype package(56) in R. The distance matrix was calculated by using the Jaccard distance metric. The following distance matrix was used to generate Multidimensional scaling (MDS) plots to visualize the differences between the groups of samples (Fig 1B and Fig S2D-S2E). In the second step, low variable genomic loci were removed from the frequency matrix, and significant differences between two groups of sample types were calculated by using a nonparametric Mann Whitney Wilcoxon test with a p-value $<0.05$ for each state separately (Fig S2F).

\section{DiffBind Analysis}

The DiffBind R package(41) was used to find differences between two groups of samples based on singular marks. For each histone modification mark, the consensus peaks across all the tumors samples were obtained using the $d b a$ and $d b a$.count function in the package with default options except for summit size, which was adjusted to 500. The matrix of the normalized reads was obtained across all the samples. Most variable bins or regions were computed with the use of the 
FSbyMAD function from the CancerSubtype(56), and the top 10,000 most variable loci were selected for further analysis. MDS plots were generated to visualize the differences between the tumor and normal samples (Fig S2A-S2B).

Similarly, a matrix of the top variable regions for H3K27AC mark (typical enhancers) was constructed across all the tumor $(n=33)$ and adenoma samples $(n=4)$ for consensus NMF (nonnegative matrix factorization) analysis. Differentially expressed regions were calculated between tumor and normal samples using dba.contrast (default settings) and dba.analyze (arguments: method=DBS_DESEQ2, bSubControl=T) function in the DiffBind package (Figure 2A).

\section{NMF clustering into Epigenomic subtypes (EpiC)}

Consensus NMF analysis was performed on the matrix of enhancers obtained from the DiffBind analysis using $n m f$ function (arguments -- rank = 2:10, nrun=1000 and method=brunet (57) from the NMF(58) package in R. Based on the dispersion, cophenetic and silhouette scores, $k$, the optimal cluster number of four was determined (Fig S7A, Fig 3A).

\section{RNA-Access Sequencing and analysis of MDACC tumor and adjacent normal samples}

mRNA libraries of the tumor $(n=7)$ and normal $(n=5)$ samples were prepared from $200 \mathrm{ng}$ of total RNA with the use of the TruSeq Stranded mRNA HT sample preparation kit. Samples were dual-indexed before pooling. Libraries were quantified by qPCR with the use of the NGS Library Quantification Kit. Pooled libraries were sequenced by using the HiSeq2000 (Illumina) according to the manufacturer's instructions. An average of approximately 30 million paired-end reads per sample were obtained. The quality of raw reads was assessed by using FastQC (https://www.bioinformatics.babraham.ac.uk/projects/fastqc/). The average per-base quality score across all of the files was greater than 32 and had passed all major tests. The raw reads were aligned to the Homo sapiens genome (hg19) using STAR v2.4.2a(59) (https://github.com/alexdobin/STAR/releases/tag/STAR_2.4.2a). The mappability of unique reads on average was $\sim 82 \%$ RNA-seq dataset. The raw counts were computed with the use of quantMode function in STAR. The read counts that were obtained are analogous to the expression level of each gene across all the samples. The differential expression analysis was done by using DESeq2(60). Genes with raw mean reads of greater than 10 were used for normalization and differential gene expression analysis using DESeq2 package in R. A Wald test, 
defined in the DESeq function of the package, was used for differential expression analysis, and shrunken log fold-changes (i.e., obtaining reliable variance estimates by pooling information across all the genes) were calculated. Genes with absolute log2 fold-change greater than $\log 2(1.5)$ and padj $<0.05$ were called as differentially expressed genes. Principal Component Analysis (PCA), MDS, hierarchical clustering plots (hclust), and XY plots between replicates of the same group or phenotype were used to examine for nominal amounts of nontechnical variation and other latent factors. PCA, hclust, and volcano plots were generated by using ggplot2 (Wickham). All of the heatmaps and box plots of differentially expressed genes were generated with the use of the pheatmap package (https://cran.rproject.org/web/packages/pheatmap/pheatmap.pdf) and ggpubr package (https://rpkgs.datanovia.com/ggpubr/), respectively.

\section{RNA-seq analyses of TCGA COAD and Adenoma dataset}

The COAD TCGA(24) RNA-seq version 2 level 3 normalized dataset (COAD.rnaseqv2_illuminahiseq_rnaseqv2_unc_edu_Level_3_RSEM_genes_normalized_ _data.data) was downloaded from http://gdac.broadinstitute.org/. Only primary tumors $(\mathrm{n}=285)$ and normal samples $(n=41)$ were used for further analysis. The normalized matrix was used for differential gene expression analysis using DESeq2 package.

The transcriptome analysis of the polyp dataset(61,62) (GEO(63) accession numbers: GSE88945, GSE106500, and GSE136044) consisting of adenoma ( $n=16)$ and normal crypts ( $n$ = 10) was performed similarly to RNA Access sequencing dataset. Briefly, the dataset was mapped to the hy19 genome using STAR aligner (v2.4.2a). The mappability of unique reads on average was $\sim 90 \%$ RNA-seq dataset. Differential expression analysis was performed using the DESeq2 package. Genes with absolute log2 fold-change greater than log2(1.5) and padj $<0.05$ were called as differentially expressed genes for both TCGA and adenoma dataset.

\section{HiChIP Analysis}

HiChIP was performed as described(64). Briefly, 1 × 10 $10^{7}$ SW480 cells were crosslinked. In situ contacts were generated in isolated and pelleted nuclei by DNA digestion with Mbol restriction enzyme, followed by biotinylation of digested DNA fragments with biotin-dATP, dCTP, dGTP, and dTTP. Thereafter, DNA was sheared with Covaris E220 with the following parameters: fill level = 
10 , duty cycle $=5, \mathrm{PIP}=140$, cycles $/$ burst $=200$, and time $=4 \mathrm{~min}$; chromatin immunoprecipitation was done for H3K27Ac with use of anti-H3K27ac antibody. After reverse-crosslinking, $150 \mathrm{ng}$ of eluted DNA was taken for biotin capture with Streptavidin C1 beads followed by transposition with Tn5. In addition, transposed DNA was used for library preparation with Nextera Ad1_noMX, Nextera Ad2.X primers, and Phusion HF 2XPCR master mix.

The following PCR program was performed: $72{ }^{\circ} \mathrm{C}$ for $5 \mathrm{~min}, 98{ }^{\circ} \mathrm{C}$ for $1 \mathrm{~min}$, then 11 cyclesat $98{ }^{\circ} \mathrm{C}$ for $15 \mathrm{~s}, 63{ }^{\circ} \mathrm{C}$ for $30 \mathrm{~s}$, and $72{ }^{\circ} \mathrm{C}$ for $1 \mathrm{~min}$. Afterward, libraries were two-sided size selected with AMPure XP beads. Finally, libraries were paired-end sequenced with reading lengths of 76 nucleotides. HiChIP paired-end reads were aligned to the hg19 genome; duplicate reads were removed, assigned to $\mathrm{Mbol}$ restriction fragments, and filtered for valid interactions. Interaction matrices were generated with HiC-Pro(65). HiC-Pro filtered reads were then processed, and loops were identified with the use of hichipper(66).

\section{C-qPCR Validation}

Chromosome conformation capture $(3 \mathrm{C})$ was performed as previously described by Hagège et al. (67) and Fullwood et al. (68) for SW480 cells. Briefly, $1 \times 10^{7}$ cells were crosslinked with $1 \%$ formaldehyde and quenched with $125 \mathrm{mM}$ glycine; the obtained nuclei and a bacterial artificial chromosome (BAC) clone were subsequently digested with $400 \mathrm{U}$ of Drall, followed by ligation, reverse-crosslinking, and DNA purification. Drall, a 6-base cutter, was selected because it allowed for individual analysis of the designated enhancers (ASCL2 E1-E4) and was deemed appropriate for the large locus size. The BAC DNA (source: CHORI) spanned the entire locus of interest and served as a control template.

After the generation of the $3 \mathrm{C}$ libraries, RT-qPCR was used to determine the relative interaction frequencies. $3 \mathrm{C}$ primers were designed according to previous guidelines(69). Genomic coordinates for the active enhancers of ASCL2 were filtered by H3K27ac peaks; test primers for the four predicted enhancers were selectively designed based on this information. A random test primer was designed at a Dralll site upstream of E1 (designated T1). Test primers were coupled with an anchor primer that represented the ASCL2 TSS to assess enhancer-promoter interactions. A gene desert (i.e., "gene poor") within chromosome 11 was used as an internal control to normalize $3 \mathrm{C}-\mathrm{qPCR}$ data. Normalized relative interaction was calculated as $2^{-\Delta \Delta \mathrm{Ct}}=\left[\left(\mathrm{C}_{\mathrm{t}}\right.\right.$ interaction $-C_{t}$ gene desert $\left.)_{3 C}-\left(C_{t \text { interaction }}-C_{t \text { gene desert }}\right)_{B A C}\right]$. 


\section{Motif analysis and TF enrichment}

We performed de novo motif discovery and a known matching motif with the use of the HOMER(25) pipeline (http://homer.ucsd.edu/homer/motif/) using default settings and background file that consisted of all the peaks or genomic regions called in the analysis. For calling the enriched TFs for Figure 11, the background file consisted of all the peaks called in CRC tumor and normal samples whereas, for Figure 3D, the background file consisted of the enriched differentially expressed regions across all the four EPIC clusters.

\section{Peak length analysis}

All peaks (called by MACS2 at $\mathrm{p}<0.01$ ) less that $1 \mathrm{~kb}$ apart within a group (Epic3 and Epic4) were merged before generating a list of peaks that were present in $100 \%$ of samples within that group. For fig. 3D, we generated a kernel density estimate (KDE) of the average peak sizes (across samples) by group to assess peak length distribution. For fig. 3F, we filtered peaks based on average peak size across samples to generate bar plots of broad peaks (left, $>5 \mathrm{~kb}$ ) and sharp peaks (right, <3kb)

\section{UpSetR}

All the ChIP-seq peaks from the H3K27ac mark were associated with the nearest gene, and then the UpSetR(70) package was used to visualize intersections of these genes across the tumor and cell lines.

\section{Pathway Analysis}

Enhancers were assigned to the genes using GREAT(17) and previously published enhancertarget networks inferred by JEME from the ENCODE+Roadmap and FANTOM5 dataset(18). Pathway analysis was performed using the Consensus Molecular Database Pathway analysis tool(71).

\section{CMS2 Classification into EpiC3 and EpiC4}


115 tumor profiles were identified as CMS2-epithelial/canonical subtype on Illumina HiSeq RNASeq data from The Cancer Genome Atlas (TCGA). EpiC 3 and EpiC 4 genes were determined from enhancer-gene annotations as described above under 'pathway analysis'. We downloaded raw gene expression data and apply across-sample normalization based on variance-stabilizing transformation (VST) (60). Three molecular subtypes were validated based on cophenetic scores in 115 cancer cases using 2098 EpiC 3 / EpiC 4 gene signatures for consensus non-negative matrix factorization (NMF) clustering. Clinical data for all profiles were downloaded from the TCGA data portal and the prognosis of each cluster was examined by Kaplan-Meier overall survival estimators. The survival outcomes of each cluster were compared by log-rank tests (survminer $R$ package). Survival analysis between cluster 2 and cluster 3 (100 total samples out of 115 samples) revealed reduced overall survival for cluster 3.

\section{Enhancer modulation using CRISPR-dCas9-KRAB}

In order to modulate gene expression without altering the target DNA sequences, an RNA-guided, catalytically inactive Cas9 (dCas9) fused to a transcriptional repressor domain (KRAB) was used to silence genomic regions identified as enhancers via KRAB repression at the promoter region. To generate a dCas9-KRAB effector stable cell line, we produced lentiviral particles from pHAGE EF1a dCas9-KRAB (Addgene plasmid \# 50919) or pHAGE TRE dCas9-KRAB (Addgene plasmid \# 50917) using a standard protocol. Transduced cells were selected for 6 days with the use of antibiotic resistance and were expanded to generate a stable cell line.

Next, gRNAs were designed by using the GPP Web Portal of the Broad Institute. Annealed gRNA oligos were ligated to pLKO.1-puro U6 sgRNA BfuAl stuffer (Addgene plasmid \# 50920) or to pLenti SpBsmBI sgRNA Hygro (Addgene plasmid \# 62205), and lentiviral particles were generated. A transduction procedure was performed in the stable dCas9-KRAB cell line, and transduced cells having both dCas9-KRAB and gRNA constructs were selected with the use of antibiotic resistance.

To evaluate the effects of the recruitment of dCas9-KRAB to the target enhancer's genomic region, H3K27ac or H3K4me1 ChIP followed by quantitative PCR for enhancer regions was performed to assess the enrichment level of H3K27ac or H3K4me1 at the enhancer site in modulated cells compared with the non-modulated parental control cells. To investigate the impact of enhancers' modulation on the corresponding gene expression, qRT-PCR was performed for the target gene. 


\section{Proliferation and invasion assays}

Cells were seeded in $180 \mu$ of medium in 96-well plates. Plates were maintained at standard culture conditions at $37^{\circ} \mathrm{C}$ without $\mathrm{CO}_{2}$ and then $20 \mu \mathrm{l}$ of alamarBlue (Sigma) was added to the cells. The magnitude of the reduction of alamarBlue correlates with the number of viable cells. Fluorescence of each sample was measured at an excitation wavelength of $560 \mathrm{~nm}$ and emission of $590 \mathrm{~nm}$ with a Tecan Infinite 200 spectrophotometer at various time points $(0,24,48,72,96$, $120 \mathrm{~h})$.

Cell invasion was assessed by using a Tumor Invasion System, 24-Multiwell Insert Plate (BD Biosciences). A BD Falcon FluoroBlok 24-Multiwell Insert System was used as a control for cell migration. This assay was performed according to the manufacturer's protocol. Fluorescence was measured after $24 \mathrm{~h}$ by using a Tecan Infinite 200 microplate reader at an excitation/emission of $494 \mathrm{~nm} / 517 \mathrm{~nm}$. Experiments were performed in triplicate.

\section{In vitro small molecule inhibitor assays and combination therapies}

Small molecule inhibitors, as shown in various experiments in Figure 4, and supplementary figures S10, S11, S12, S13 and S14 were used in CRC cell lines. Inhibitors were used in final concentrations of $100 \mathrm{pM}, 1 \mathrm{nM}, 10 \mathrm{nM}, 100 \mathrm{nM}, 1 \mu \mathrm{M}$, and $10 \mathrm{uM}$. A cell viability assay was performed as described previously(72,73). $I \mathrm{C}_{50}$ and $\mathrm{AUC}$ were calculated for each compound in each of the cell lines. Based on these indices, the most potent/efficient compounds were used for combinatorial treatments.

\section{Mouse experiments}

Patient-derived xenografts: Primary human-tumor xenograft models were established as previously described(74). Tumor specimens were obtained from patients with metastatic colorectal cancer being treated at The University of Texas MD Anderson Cancer Center, and all patients provided informed written consent for specimens to be used for research purposes including implantation in xenografts. Samples were obtained with the approval of the Institutional Review Board. Xenografts were established in NOD/SCID 8-week-old female mice. Once established, PDXs were expanded in 69 athymic nude mice for experiments.

After tumors were established with the median tumor volume exceeding $300 \mathrm{~mm}^{3}$, 
treatment of 3-6 mice per arm was commenced via oral gavage with either vehicle control $(0.5 \%$ Tween 80/0.5\% CMC in water), binimetinib (3 mpk, BID), trametinib (0.25 mpk, QD), iBET-151 (15 mpk, QD), or drug combinations as indicated in Figures 2I-2J. Tumor size and mouse weight were measured twice a week. After 21 days, treatment was discontinued, and mice were euthanized. Tumors from 3 mice per arm were excised (2 - 4 hours after treatment), segmented, and immediately flash-frozen in liquid nitrogen or in $10 \%$ buffered formalin solution.

Cell line xenografts: HCT116 (CMS1), T84 (CMS2), LS513 (CMS3), and SM620 (CMS4) were grown in the manufacturer-recommended media. One million cells were transplanted per site, and 5 mice were injected per experimental arm. Once tumors reached $\sim 5 \mathrm{~mm}$ in size, the treatment was started as indicated in the specific figures/legends. All drugs were purchased from commercial sources: i-BET151 (MedChemExpress, 15mg/kg, every other day), gefitinib (EGFRi, Sigma, $100 \mathrm{mg} / \mathrm{kg}$, every other day), SB431542 (TGF $\beta \mathrm{i}$, MedChemExpress, 10mg/kg, every other day), and olaparib (APExBio, 50mg/kg, every other day) and dissolved in the manufacturerrecommended vehicles. 30\% PEG-300 was used as the vehicle control. Tumor volume was measured every other day. Once tumors reached $1.5 \mathrm{~cm}$ in one direction in any of the experimental arms, the mice were euthanized and the tumors were harvested.

\section{Production of anti-LGR5 mAb}

Cloning and production of anti-LGR5 (8F2) mAb rat/mouse IgG2a chimera was performed as previously reported (75). 


\section{Generation of anti-LGR5 mAb-RVX-208 conjugate}

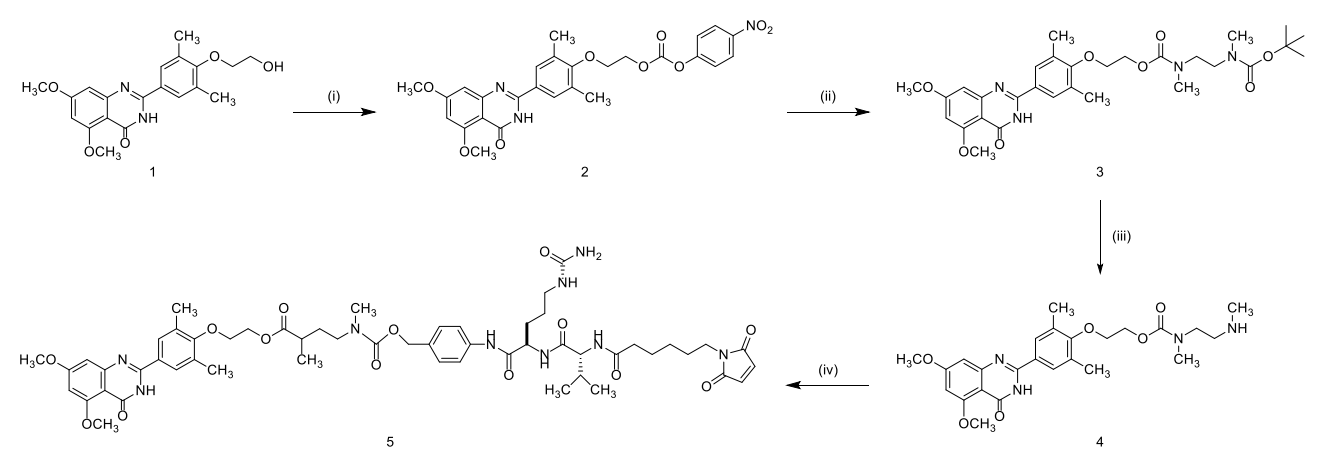

Scheme 1: (i) Bis (4-nitrophenyl) carbonate, $i-\operatorname{Pr}_{2} N E t$, DMF, r.t., overnight stirring; (ii) tert-butyl methyl [2-(methylamino) ethyl]carbamate, 2,6-lutidine, $45^{\circ} \mathrm{C}$, overnight stirring; (iii) $\mathrm{TFA}, \mathrm{CH}_{2} \mathrm{Cl}_{2}$, r.t., 2 h stirring; (iv) MC-Val-Cit-PAB-PNP, $i-\operatorname{Pr}_{2} \mathrm{NEt}, 37^{\circ} \mathrm{C}$, overnight stirring.

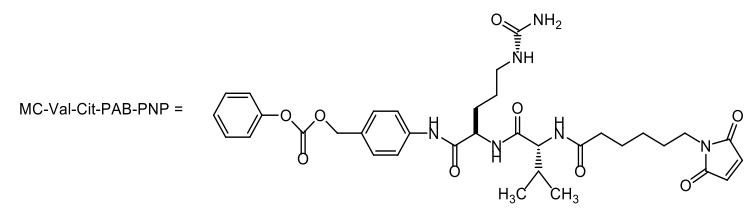

Synthesis of 2-(4-(5,7-dimethoxy-4-oxo-3,4-dihydroquinazolin-2-yl)-2,6dimethylphenoxy)ethyl (4-nitrophenyl) carbonate (2). A solution of RVX-208 (1) (20 mg, 0.054 $\mathrm{mmol}$ ) and bis(4-nitrophenyl) carbonate $(50 \mathrm{mg}, 0.16 \mathrm{mmol}$ ) in anhydrous $N, N$-dimethylformamide (DMF) (3 mL) was mixed with $25 \mu \mathrm{L} \mathrm{N}, N$-diisopropylethylamine $(17 \mathrm{mg}, 0.16 \mathrm{mmol}$ ) and stirred room temperature (r.t.) overnight. The reaction mixture was evaporated and purified by column chromatography $\left(\mathrm{SiO}_{2} ; 95 \%(\mathrm{vol} / \mathrm{vol}) \mathrm{CH}_{2} \mathrm{Cl}_{2} / \mathrm{MeOH}\right)$ to give the expected product with high yield (23 $\mathrm{mg}, 70 \%$ ). The product was verified by mass spectra analysis. MS, ESI+: $\mathrm{m} / \mathrm{z}$ calculated for $\mathrm{C}_{27} \mathrm{H}_{25} \mathrm{~N}_{3} \mathrm{O}_{9}, 535.16$; found $\mathrm{m} / \mathrm{z}, 536.00(\mathrm{M}+\mathrm{H})^{+}$.

\section{Synthesis of tert-butyl (2-(4-(5,7-dimethoxy-4-oxo-3,4-dihydroquinazolin-2-yl)-2,6-} dimethylphenoxy)ethyl) ethane-1,2-diylbis(methylcarbamate) (3). A solution of compound 2 (30 mg, $0.028 \mathrm{mmol}$ ) and tert-butylmethyl [2-(methyl amine) ethyl] carbonate (30 mg, 1.2 equivalent, $0.067 \mathrm{mmol})$ was prepared in anhydrous DMF $(2 \mathrm{~mL})$ and heated $a 5^{\circ} \mathrm{C}$. To the reaction mixture, 1-hydroxy-7-azabenzotriazole (HOAt) $(10 \mathrm{mg}, 0.073)$ and 2,6-lutidine (23 mg, 
$0.21 \mathrm{mmol}$ ) were added and heated overnight with continuous stirring. Following evaporation of $\mathrm{DMF}$, the crude product was purified by column chromatography $\left(\mathrm{SiO}_{2} ; 98 \%\right.$ (vol/vol) $\mathrm{CH}_{2} \mathrm{Cl}_{2} / \mathrm{MeOH}$ ) to yield $28 \mathrm{mg}$ of 3 (84\% yield). MS, ESI+: $\mathrm{m} / \mathrm{z}$ calculated for $\mathrm{C}_{30} \mathrm{H}_{40} \mathrm{~N}_{4} \mathrm{O}_{8}, 584.28$; found $m / z, 584.9(\mathrm{M}+\mathrm{H})^{+}$.

Synthesis of 2-(4-(5,7-dimethoxy-4-oxo-3,4-dihydroquinazolin-2-yl)-2,6dimethylphenoxy)ethyl methyl(2-(methylamino)ethyl)carbamate (4). Trifluroacetic acid (1 $\mathrm{mL}$ ) was added to a solution of compound $3(28 \mathrm{mg}, 0.047 \mathrm{mmol})$ in anhydrous dichloromethane (2 $\mathrm{mL}$ ) under $\mathrm{N}_{2}$ atmosphere and stirred at room temperature. After $2 \mathrm{~h}$, the solvent was evaporated and the reaction mixture was washed with diethyl ether and dried to give compound 4 (21 mg, $90 \%$ yield). MS, ESI+: $\mathrm{m} / \mathrm{z}$ calculated for $\mathrm{C}_{25} \mathrm{H}_{32} \mathrm{~N}_{4} \mathrm{O}_{6}, 484.23$; found $\mathrm{m} / \mathrm{z}$, 485.00 $(\mathrm{M}+\mathrm{H})^{+}$.

Synthesis of RVX-208- MC-Val-Cit-PAB-PNP-maleimide (5). To a $2 \mathrm{~mL}$ solution of anhydrous DMF containing MC-VC-PAB-PNP (30 mg, $0.0413 \mathrm{mmol}$ ) and compound 4 (20 mg, $0.0413 \mathrm{mmol}$ ), $30 \mu \mathrm{L}$ of $N, N$-diisopropylethylamine $(20 \mathrm{mg}, 0.19 \mathrm{mmol})$ was added and the reaction was heated at $37^{\circ} \mathrm{C}$ overnight. The crude product was purified on HPLC and to give $24 \mathrm{mg}$ of 5 (55 \% yield). MS (ES) m/z: 1082.8[M+H] $]^{+} ; 1104.9[\mathrm{M}+\mathrm{Na}]^{+} \mathrm{MS}, \mathrm{ESI}+: \mathrm{m} / \mathrm{z}$ calculated for $\mathrm{C}_{54} \mathrm{H}_{70} \mathrm{~N}_{10} \mathrm{O}_{14}, 1082.51$; found $m / z, 1082.8(\mathrm{M}+\mathrm{H})^{+}, 1104.9(\mathrm{M}+\mathrm{Na})^{+}$.

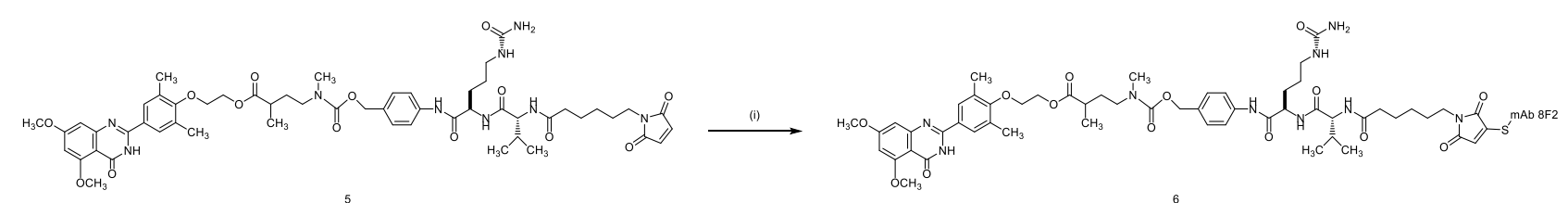

Scheme 2: (i) Reduced 8F2, DMSO, $\mathrm{Na}_{2} \mathrm{PO}_{4}$ buffer ( $\mathrm{pH} 8.2$ ), 3 h at r.t., then $4^{\circ}$ overnight.

Synthesis of anti-LGR5 (8F2) antibody-drug conjugate (6). To a solution of 8F2-mAb (270 $\mu$, $0.0018 \mu \mathrm{mol})$ in PBS $(170 \mu \mathrm{L}), 5$ molar equivalent of DTT $\left(30 \mu \mathrm{g}, 0.19 \mu \mathrm{mol}, 30 \mu \mathrm{L} \mathrm{H}_{2} \mathrm{O}\right)$ was added to partially cleave the interchain disulfides. The reaction was warmed to $37^{\circ} \mathrm{C}$ and proceeded for $3 \mathrm{~h}$. After cooling to r.t., the mixture was purified with a Zeba desalting spin column in $\mathrm{Na}_{2} \mathrm{PO}_{4}(\mathrm{pH}$ 8.2) buffer at a concentration of $1 \mathrm{mg} / \mathrm{mL}$. A 10 molar equivalent of 5 (20 $\mu \mathrm{g}, 30$ $\mu \mathrm{L}$ ) in DMSO was added to the mAb solution and continuously stirred for $3 \mathrm{~h}$ at r.t., and then at 
bioRxiv preprint doi: https://doi.org/10.1101/2020.09.04.283838; this version posted September 4, 2020. The copyright holder for this preprint

(which was not certified by peer review) is the author/funder, who has granted bioRxiv a license to display the preprint in perpetuity. It is made available under aCC-BY-NC-ND 4.0 International license.

$4^{\circ} \mathrm{C}$ overnight. The resulting ADC, 6 , was purified with a Zeba desalting spin column and collected in PBS at a final concentration of $(1 \mathrm{mg} / \mathrm{mL})$. 


\section{REFERENCES}

1. Strum WB. Colorectal Adenomas. The New England journal of medicine 2016;374(11):1065-75 doi 10.1056/NEJMra1513581.

2. Fearon ER, Vogelstein B. A genetic model for colorectal tumorigenesis. Cell 1990;61(5):759-67.

3. Guinney J, Dienstmann R, Wang X, de Reynies A, Schlicker A, Soneson C, et al. The consensus molecular subtypes of colorectal cancer. Nature medicine 2015;21(11):13506 doi 10.1038/nm.3967.

4. Haan JC, Labots M, Rausch C, Koopman M, Tol J, Mekenkamp LJ, et al. Genomic landscape of metastatic colorectal cancer. Nature communications 2014;5:5457 doi $10.1038 /$ ncomms6457.

5. TCGA. Comprehensive molecular characterization of human colon and rectal cancer. Nature 2012;487(7407):330-7 doi 10.1038/nature11252.

6. Yaeger R, Chatila WK, Lipsyc MD, Hechtman JF, Cercek A, Sanchez-Vega F, et al. Clinical Sequencing Defines the Genomic Landscape of Metastatic Colorectal Cancer. Cancer cell 2018;33(1):125-36 e3 doi 10.1016/j.ccell.2017.12.004.

7. Terasaki H, Saitoh T, Shiokawa K, Katoh M. Frizzled-10, up-regulated in primary colorectal cancer, is a positive regulator of the WNT - beta-catenin - TCF signaling pathway. Int J Mol Med 2002;9(2):107-12.

8. Loven J, Hoke HA, Lin CY, Lau A, Orlando DA, Vakoc CR, et al. Selective inhibition of tumor oncogenes by disruption of super-enhancers. Cell 2013;153(2):320-34 doi 10.1016/j.cell.2013.03.036.

9. Rai K, Sarkar S, Broadbent TJ, Voas M, Grossmann KF, Nadauld LD, et al. DNA demethylase activity maintains intestinal cells in an undifferentiated state following loss of APC. Cell 2010;142(6):930-42 doi 10.1016/j.cell.2010.08.030.

10. Tao Y, Kang B, Petkovich DA, Bhandari YR, In J, Stein-O'Brien G, et al. Aging-like Spontaneous Epigenetic Silencing Facilitates Wnt Activation, Stemness, and Braf(V600E)-Induced Tumorigenesis. Cancer cell 2019;35(2):315-28 e6 doi 10.1016/j.ccell.2019.01.005.

11. Akhtar-Zaidi B, Cowper-Sal-lari R, Corradin O, Saiakhova A, Bartels CF, Balasubramanian D, et al. Epigenomic enhancer profiling defines a signature of colon cancer. Science 2012;336(6082):736-9 doi 10.1126/science.1217277. 
12. Cohen AJ, Saiakhova A, Corradin O, Luppino JM, Lovrenert K, Bartels CF, et al. Hotspots of aberrant enhancer activity punctuate the colorectal cancer epigenome. Nature communications 2017;8:14400 doi 10.1038/ncomms14400.

13. Hung S, Saiakhova A, Faber ZJ, Bartels CF, Neu D, Bayles I, et al. Mismatch repairsignature mutations activate gene enhancers across human colorectal cancer epigenomes. eLife 2019;8 doi 10.7554/eLife.40760.

14. Ernst J, Kellis M. ChromHMM: automating chromatin-state discovery and characterization. Nature methods 2012;9(3):215-6 doi 10.1038/nmeth.1906.

15. Yen A, Kellis M. Systematic chromatin state comparison of epigenomes associated with diverse properties including sex and tissue type. Nature communications 2015;6:7973 doi 10.1038/ncomms8973.

16. Whyte WA, Orlando DA, Hnisz D, Abraham BJ, Lin CY, Kagey MH, et al. Master transcription factors and mediator establish super-enhancers at key cell identity genes. Cell 2013;153(2):307-19 doi 10.1016/j.cell.2013.03.035.

17. McLean CY, Bristor D, Hiller M, Clarke SL, Schaar BT, Lowe CB, et al. GREAT improves functional interpretation of cis-regulatory regions. Nature biotechnology 2010;28(5):495501 doi 10.1038/nbt.1630.

18. Cao Q, Anyansi C, Hu X, Xu L, Xiong L, Tang W, et al. Reconstruction of enhancer-target networks in 935 samples of human primary cells, tissues and cell lines. Nature genetics 2017;49(10):1428-36 doi 10.1038/ng.3950.

19. Schuijers J, Junker JP, Mokry M, Hatzis P, Koo BK, Sasselli V, et al. Ascl2 acts as an Rspondin/Wnt-responsive switch to control stemness in intestinal crypts. Cell Stem Cell 2015;16(2):158-70 doi 10.1016/j.stem.2014.12.006.

20. Zhu R, Yang Y, Tian Y, Bai J, Zhang X, Li X, et al. Ascl2 knockdown results in tumor growth arrest by miRNA-302b-related inhibition of colon cancer progenitor cells. PloS one 2012;7(2):e32170 doi 10.1371/journal.pone.0032170.

21. Rout A, Sukhi A, Chaudhary R, Bliden KP, Tantry US, Gurbel PA. Investigational drugs in phase II clinical trials for acute coronary syndromes. Expert Opin Investig Drugs 2020;29(1):33-47 doi 10.1080/13543784.2020.1708324.

22. Eide PW, Bruun J, Lothe RA, Sveen A. CMScaller: an R package for consensus molecular subtyping of colorectal cancer pre-clinical models. Sci Rep 2017;7(1):16618 doi 10.1038/s41598-017-16747-x. 
23. Chen X, Halberg RB, Burch RP, Dove WF. Intestinal adenomagenesis involves core molecular signatures of the epithelial-mesenchymal transition. J Mol Histol 2008;39(3):283-94 doi 10.1007/s10735-008-9164-3.

24. Cancer Genome Atlas N. Comprehensive molecular characterization of human colon and rectal cancer. Nature 2012;487(7407):330-7 doi 10.1038/nature11252.

25. Heinz S, Benner C, Spann N, Bertolino E, Lin YC, Laslo P, et al. Simple combinations of lineage-determining transcription factors prime cis-regulatory elements required for macrophage and $B$ cell identities. Molecular cell 2010;38(4):576-89 doi 10.1016/j.molcel.2010.05.004.

26. Lee KS, Kwak Y, Nam KH, Kim DW, Kang SB, Choe G, et al. Favorable prognosis in colorectal cancer patients with co-expression of c-MYC and ss-catenin. BMC Cancer 2016;16(1):730 doi 10.1186/s12885-016-2770-7.

27. Smith DR, Goh HS. Overexpression of the c-myc proto-oncogene in colorectal carcinoma is associated with a reduced mortality that is abrogated by point mutation of the p53 tumor suppressor gene. Clinical cancer research : an official journal of the American Association for Cancer Research 1996;2(6):1049-53.

28. Erisman MD, Rothberg PG, Diehl RE, Morse CC, Spandorfer JM, Astrin SM. Deregulation of c-myc gene expression in human colon carcinoma is not accompanied by amplification or rearrangement of the gene. Molecular and cellular biology 1985;5(8):1969-76 doi 10.1128/mcb.5.8.1969.

29. Rochlitz CF, Herrmann R, de Kant E. Overexpression and amplification of c-myc during progression of human colorectal cancer. Oncology 1996;53(6):448-54 doi 10.1159/000227619.

30. Belaguli NS, Aftab M, Rigi M, Zhang M, Albo D, Berger DH. GATA6 promotes colon cancer cell invasion by regulating urokinase plasminogen activator gene expression. Neoplasia 2010;12(11):856-65.

31. Whissell G, Montagni E, Martinelli P, Hernando-Momblona X, Sevillano M, Jung P, et al. The transcription factor GATA6 enables self-renewal of colon adenoma stem cells by repressing BMP gene expression. Nat Cell Biol 2014;16(7):695-707 doi 10.1038/ncb2992.

32. Martinelli P, Carrillo-de Santa Pau E, Cox T, Sainz B, Jr., Dusetti N, Greenhalf W, et al. GATA6 regulates EMT and tumour dissemination, and is a marker of response to adjuvant chemotherapy in pancreatic cancer. Gut 2017;66(9):1665-76 doi 10.1136/gutjnl-2015311256. 
33. Prevostel C, Blache P. The dose-dependent effect of SOX9 and its incidence in colorectal cancer. Eur J Cancer 2017;86:150-7 doi 10.1016/j.ejca.2017.08.037.

34. Prevostel C, Rammah-Bouazza C, Trauchessec H, Canterel-Thouennon L, Busson M, Ychou M, et al. SOX9 is an atypical intestinal tumor suppressor controlling the oncogenic Wnt/ss-catenin signaling. Oncotarget 2016;7(50):82228-43 doi 10.18632/oncotarget.10573.

35. Lu B, Fang Y, Xu J, Wang L, Xu F, Xu E, et al. Analysis of SOX9 expression in colorectal cancer. Am J Clin Pathol 2008;130(6):897-904 doi 10.1309/AJCPW1W8GJBQGCNI.

36. Cheng JM, Yao MR, Zhu Q, Wu XY, Zhou J, Tan WL, et al. Silencing of stat4 gene inhibits cell proliferation and invasion of colorectal cancer cells. J Biol Regul Homeost Agents 2015;29(1):85-92.

37. Slattery ML, Lundgreen A, Kadlubar SA, Bondurant KL, Wolff RK. JAK/STAT/SOCSsignaling pathway and colon and rectal cancer. Mol Carcinog 2013;52(2):155-66 doi 10.1002/mc.21841.

38. Herbert K, Binet R, Lambert JP, Louphrasitthiphol P, Kalkavan H, Sesma-Sanz L, et al. BRN2 suppresses apoptosis, reprograms DNA damage repair, and is associated with a high somatic mutation burden in melanoma. Genes \& development 2019;33(5-6):310-32 doi 10.1101/gad.314633.118.

39. Simmons JL, Pierce CJ, Al-Ejeh F, Boyle GM. MITF and BRN2 contribute to metastatic growth after dissemination of melanoma. Sci Rep 2017;7(1):10909 doi 10.1038/s41598017-11366-y.

40. Dai L, Cui X, Zhang X, Cheng L, Liu Y, Yang Y, et al. SARI inhibits angiogenesis and tumour growth of human colon cancer through directly targeting ceruloplasmin. Nature communications 2016;7:11996 doi 10.1038/ncomms11996.

41. Sveen A, Bruun J, Eide PW, Eilertsen IA, Ramirez L, Murumagi A, et al. Colorectal Cancer Consensus Molecular Subtypes Translated to Preclinical Models Uncover Potentially Targetable Cancer Cell Dependencies. Clinical cancer research : an official journal of the American Association for Cancer Research 2018;24(4):794-806 doi 10.1158/10780432. CCR-17-1234.

42. Linnekamp JF, Hooff SRV, Prasetyanti PR, Kandimalla R, Buikhuisen JY, Fessler E, et al. Consensus molecular subtypes of colorectal cancer are recapitulated in in vitro and in vivo models. Cell Death Differ 2018;25(3):616-33 doi 10.1038/s41418-017-0011-5. 
43. Corces MR, Granja JM, Shams S, Louie BH, Seoane JA, Zhou W, et al. The chromatin accessibility landscape of primary human cancers. Science 2018;362(6413) doi 10.1126/science.aav1898.

44. Xu C, Tian G, Jiang C, Xue H, Kuerbanjiang M, Sun L, et al. NPTX2 promotes colorectal cancer growth and liver metastasis by the activation of the canonical Wnt/beta-catenin pathway via FZD6. Cell Death Dis 2019;10(3):217 doi 10.1038/s41419-019-1467-7.

45. Amit M, Takahashi H, Dragomir MP, Lindemann A, Gleber-Netto FO, Pickering CR, et al. Loss of p53 drives neuron reprogramming in head and neck cancer. Nature 2020;578(7795):449-54 doi 10.1038/s41586-020-1996-3.

46. Khvorova A, Watts JK. The chemical evolution of oligonucleotide therapies of clinical utility. Nature biotechnology 2017;35(3):238-48 doi 10.1038/nbt.3765.

47. Terranova C, Tang M, Orouji E, Maitituoheti M, Raman A, Amin S, et al. An Integrated Platform for Genome-wide Mapping of Chromatin States Using High-throughput ChIP. sequencing in Tumor Tissues. Journal of visualized experiments : JoVE 2018(134) doi $10.3791 / 56972$.

48. Gausachs M, Borras E, Chang K, Gonzalez S, Azuara D, Delgado Amador A, et al. Mutational Heterogeneity in APC and KRAS Arises at the Crypt Level and Leads to Polyclonality in Early Colorectal Tumorigenesis. Clinical cancer research : an official journal of the American Association for Cancer Research 2017;23(19):5936-47 doi 10.1158/1078-0432.CCR-17-0821.

49. Garber M, Yosef N, Goren A, Raychowdhury R, Thielke A, Guttman M, et al. A highthroughput chromatin immunoprecipitation approach reveals principles of dynamic gene regulation in mammals. Molecular cell 2012;47(5):810-22 doi 10.1016/j.molcel.2012.07.030.

50. S. A. FastQC: a quality control tool for high throughput sequence data. . 2010.

51. Langmead B, Trapnell C, Pop M, Salzberg SL. Ultrafast and memory-efficient alignment of short DNA sequences to the human genome. Genome biology 2009;10(3):R25 doi 10.1186/gb-2009-10-3-r25.

52. Faust GG, Hall IM. SAMBLASTER: fast duplicate marking and structural variant read extraction. Bioinformatics 2014;30(17):2503-5 doi 10.1093/bioinformatics/btu314.

53. Li H, Handsaker B, Wysoker A, Fennell T, Ruan J, Homer N, et al. The Sequence Alignment/Map format and SAMtools. Bioinformatics 2009;25(16):2078-9 doi 10.1093/bioinformatics/btp352. 
54. Ramirez F, Ryan DP, Gruning B, Bhardwaj V, Kilpert F, Richter AS, et al. deepTools2: a next generation web server for deep-sequencing data analysis. Nucleic acids research 2016;44(W1):W160-5 doi 10.1093/nar/gkw257.

55. Zhang Y, Liu T, Meyer CA, Eeckhoute J, Johnson DS, Bernstein BE, et al. Model-based analysis of ChIP-Seq (MACS). Genome biology 2008;9(9):R137 doi 10.1186/gb-2008-99-r137.

56. Xu T, Le TD, Liu L, Su N, Wang R, Sun B, et al. CancerSubtypes: an R/Bioconductor package for molecular cancer subtype identification, validation and visualization. Bioinformatics 2017;33(19):3131-3 doi 10.1093/bioinformatics/btx378.

57. Brunet JP, Tamayo P, Golub TR, Mesirov JP. Metagenes and molecular pattern discovery using matrix factorization. Proceedings of the National Academy of Sciences of the United States of America 2004;101(12):4164-9 doi 10.1073/pnas.0308531101.

58. Gaujoux R, Seoighe C. A flexible R package for nonnegative matrix factorization. BMC bioinformatics 2010;11:367 doi 10.1186/1471-2105-11-367.

59. Dobin A, Davis CA, Schlesinger F, Drenkow J, Zaleski C, Jha S, et al. STAR: ultrafast universal RNA-seq aligner. Bioinformatics 2013;29(1):15-21 doi 10.1093/bioinformatics/bts635.

60. Love MI, Huber W, Anders S. Moderated estimation of fold change and dispersion for RNA-seq data with DESeq2. Genome biology 2014;15(12):550 doi 10.1186/s13059-0140550-8.

61. Chang K, Taggart MW, Reyes-Uribe L, Borras E, Riquelme E, Barnett RM, et al. Immune Profiling of Premalignant Lesions in Patients With Lynch Syndrome. JAMA Oncol 2018;4(8):1085-92 doi 10.1001/jamaoncol.2018.1482.

62. Crespo M, Vilar E, Tsai SY, Chang K, Amin S, Srinivasan T, et al. Colonic organoids derived from human induced pluripotent stem cells for modeling colorectal cancer and drug testing. Nature medicine 2017;23(7):878-84 doi 10.1038/nm.4355.

63. Barrett T, Wilhite SE, Ledoux P, Evangelista C, Kim IF, Tomashevsky M, et al. NCBI GEO: archive for functional genomics data sets--update. Nucleic acids research 2013;41(Database issue):D991-5 doi 10.1093/nar/gks1193.

64. Mumbach MR, Rubin AJ, Flynn RA, Dai C, Khavari PA, Greenleaf WJ, et al. HiChIP: efficient and sensitive analysis of protein-directed genome architecture. Nature methods 2016;13(11):919-22 doi 10.1038/nmeth.3999. 
65. Servant N, Varoquaux N, Lajoie BR, Viara E, Chen CJ, Vert JP, et al. HiC-Pro: an optimized and flexible pipeline for $\mathrm{Hi}-\mathrm{C}$ data processing. Genome biology 2015;16:259 doi 10.1186/s13059-015-0831-x.

66. Lareau CA, Aryee MJ. hichipper: a preprocessing pipeline for calling DNA loops from HiChIP data. Nature methods 2018;15(3):155-6 doi 10.1038/nmeth.4583.

67. Hagege H, Klous P, Braem C, Splinter E, Dekker J, Cathala G, et al. Quantitative analysis of chromosome conformation capture assays (3C-qPCR). Nature protocols 2007;2(7):1722-33 doi 10.1038/nprot.2007.243.

68. Fullwood MJ, Liu MH, Pan YF, Liu J, Xu H, Mohamed YB, et al. An oestrogen-receptoralpha-bound human chromatin interactome. Nature 2009;462(7269):58-64 doi 10.1038/nature08497.

69. Naumova N, Smith EM, Zhan Y, Dekker J. Analysis of long-range chromatin interactions using Chromosome Conformation Capture. Methods 2012;58(3):192-203 doi 10.1016/j.ymeth.2012.07.022.

70. Lex A, Gehlenborg N, Strobelt H, Vuillemot R, Pfister H. UpSet: Visualization of Intersecting Sets. IEEE transactions on visualization and computer graphics 2014;20(12):1983-92 doi 10.1109/tvcg.2014.2346248.

71. Kamburov A, Pentchev K, Galicka H, Wierling C, Lehrach H, Herwig R. ConsensusPathDB: toward a more complete picture of cell biology. Nucleic acids research 2011;39(Database issue):D712-7 doi 10.1093/nar/gkq1156.

72. Fiziev P, Akdemir KC, Miller JP, Keung EZ, Samant NS, Sharma S, et al. Systematic Epigenomic Analysis Reveals Chromatin States Associated with Melanoma Progression. Cell Rep 2017;19(4):875-89 doi 10.1016/j.celrep.2017.03.078.

73. Rai K, Akdemir KC, Kwong LN, Fiziev P, Wu CJ, Keung EZ, et al. Dual Roles of RNF2 in Melanoma Progression. Cancer discovery 2015 doi 10.1158/2159-8290.CD-15-0493.

74. Katsiampoura A, Raghav K, Jiang ZQ, Menter DG, Varkaris A, Morelli MP, et al. Modeling of Patient-Derived Xenografts in Colorectal Cancer. Mol Cancer Ther 2017;16(7):1435-42 doi 10.1158/1535-7163.MCT-16-0721.

75. Gong X, Azhdarinia A, Ghosh SC, Xiong W, An Z, Liu Q, et al. LGR5-Targeted AntibodyDrug Conjugate Eradicates Gastrointestinal Tumors and Prevents Recurrence. Mol Cancer Ther 2016;15(7):1580-90 doi 10.1158/1535-7163.MCT-16-0114. 


\section{Code availability}

The ChIP-seq analysis source code are available at the following webpage: https://doi.org/10.5281/zenodo.819971.

\section{ACKNOWLEDGEMENTS}

We thank Integromics group, the Advanced Technology Genomics Core Facility ( $\mathrm{NCl}$ Grant CA016672(ATGC), the Research Animal Support Facility, the Advanced Microscopy Core Facility (funded by NIH S10 RR029552), Functional Genomics Core (NCl Cancer Center Support Grant (P30 CA016672) and MD Anderson Cancer Center Clinical Core. This work was supported by ACS research scholar award, CPRIT IIRA award (RP200390), a Career Development Award from MDACC GI SPORE to K.R. We thank Veena Kochat, Sharon Landers and Angela Bhalla for helpful discussions and suggestions.

\section{AUTHOR CONTRIBUTIONS}

EO conceived, conceptualized and designed the study, planned and carried out experiments, analyzed data, made figures, and wrote the manuscript. ATR conceptualized and designed the study, carried out computational analysis, prepared figures, and wrote the manuscript. AKS carried out experiments, computational analysis and made figures regarding HiChIP and wrote the manuscript. A.S. performed mouse treatment experiments. EA performed computational analysis for CMS2 classification. AKG performed the $3 \mathrm{C}$ experiments and edited the manuscript. JS performed peak length analysis. MT helped with informatics analysis. CJT, SCC, MM, KT, ZJ, JSD, SG, HML, LRU, KC, YL, HC, AA provided technical help. JSM, EV, KSC and SK contributed to study design and provided reagents. KR conceived, conceptualized and designed the study, performed experiments, evaluated data, made figures and wrote the manuscript. All the authors edited the manuscript.

\section{COMPETING INTERESTS}

All the authors declare no conflict of interests. 


\section{FIGURE LEGENDS}

Fig. 1. H3K27ac-identified global enhancer pattern could differentiate colorectal cancer from normal colon.

A. Emission probabilities of ChromHMM-based 10-chromatin state calls, which is based on the global distribution of combinatorial patterns of 6 histone marks (H3K4me1, H3K27ac, H3K4me3, H3K27me3, H3K79me2, and H3K9me3) from 13 adenocarcinomas and 12 adjacent normal tissues. Each row is representative of a combinatorial chromatin state pattern, which is annotated on the right based on the constituent histone mark and neighborhood plot (Fig. S1B). Columns represent each histone mark. Colors represent the frequency of occurrence of that mark in the corresponding chromatin state on a scale of 0 (white) to 1 (blue).

B. Multidimensional scaling (MDS) plot for chromatin state 5 between normal tissues (blue) and adenocarcinoma samples (red).

C. MDS plot for global H3K27ac patterns in the adenomas (A), adenocarcinomas (T), adjacent normal colon $(\mathrm{N})$ and cancer cell lines. Adenoma samples are spread over normal and tumor samples and cannot be distinguished with use of H3K27ac. Red, Green, Blue and Purple colors were used for Tumor, Adenoma, Normal Colon and CRC cell lines respectively.

D. Venn diagram showing overlaps between total number of H3K27ac-defined active enhancer peaks in normal samples (blue), adenomas (green) and CRC tumors (red).

E. Venn diagram showing overlaps between total number of super-enhancer peaks (called using ROSE) in normal samples (blue), adenomas (green) and CRC tumors (red).

F. List of significantly enriched pathways in genes targeted by enhancers specific to CRC tumors $(T)$, adenomas $(A)$, and normal colon tissue $(N)$. $q$-values are shown and are color-coded based on the level of significance.

G. Integrative Genomics Viewer (IGV) images showing enrichment of H3K27ac peaks around ASCL2, SALL4 and FZD10 genes using aggregate ChIP-seq profiles of normal colon, adenoma and CRC tumors.

H. Violin plots showing mRNA expression levels for ASCL2, SALL4 and FZD10 in adenomas, the adenocarcinoma cohort used in this study (labeled as MDACC) and TCGA adenocarcinoma cohort. The reported p-values were calculated using the DESeq2 (60).

I. List of enriched transcription factor (TF) motifs in CRC- or normal tissue-specific active enhancers. Motifs are identified using $\operatorname{HOMER}(25)$ on the basis of the shared H3K27ac peak in each group. 
Fig. 2. Inactivation of top tumor-specific enhancers leads to transcriptional downregulation and loss of cell proliferation.

A. Volcano plot showing top CRC-enriched typical enhancers ranked based on fold-change and p-value. Top de novo gained enhancers among tumor $(n=33)$ as compared to adjacent normal $(n=15)$ samples are annotated according to their associated genes. Thresholds of $\log 2 \mathrm{FC}>1$ and FDR $<0.05$ are used to annotate de novo enhancer peaks. ASCL2 and FZD10 enhancers are among this subset of highly enriched regions. There are two top ranked enhancer peaks around FZD10 gene.

B. Interaction map of active enhancers $(E)$ with the gene promoter $(P)$ around the ASCL2 locus as determined in SW480 cell line by HiChIP. Interactions were observed in a super-enhancer (E1-E3) region located $\sim 70 \mathrm{~kb}$ upstream of ASCL2 promoter (PET counts=9, $q$-value $=3.2 \mathrm{e}^{-07}$ ). These regions are among the highly enriched tumor-specific enhancers and were present in multiple CRC cell lines (Figure S4A).

C. Bar graph showing relative enrichment of H3K27ac as derived from a ChIP-qPCR experiment on E1-E3 for ASCL2 super-enhancer (from panel B) in SW480 parental or modified cells harboring gRNAs to these enhancers. Y-axis represents fold change in intensity of H3K27ac signal for each enhancer in gRNA harboring cell in comparison to the parental cell. Asterisk * denotes $p$-value $<0.05$.

D. Bar graph showing relative ASCL2 mRNA expression in E1-E3 gRNAs for ASCL2 superenhancer harboring SW480 cells in comparison to parental cells (ASCL2_INTACT).

E. Growth curve showing relative viability of SW480 cells harboring E1-E3 for ASCL2 superenhancer in comparison to parental cell (ASCL2_INTACT) showing lower rates of proliferation in cells with ASCL2-inactivated enhancers.

F. Growth curves for LGR5-silenced SW480 cells (SW480 ${ }^{\text {LGR5-KD }) ~ i n ~ c o m p a r i s o n ~ t o ~ t h e ~ p a r e n t a l ~}$ cells upon treatment with increasing concentrations of two preparations of antibody-drug conjugate (ADC) with bromodomain inhibitor (RVX208) conjugated to LGR5 antibody (ADCPrep1 and ADC-Prep2) and RVX-208 alone. Y-axis shows percent viability of treated cells in comparison to control (DMSO) treated cells.

G. Growth curves for a patient-derived CRC organoid upon treatment with LGR5-RVX208 conjugate $(A D C)$. Y-axis shows percent viability of treated cells in comparison to control (DMSO) treated cells.

H. Tumor volume curves for C1138, F3053, F3008, and B8131 on treatment with MEK inhibitors (trametinib), BRD inhibitor (i-BET151), and a combination of MEK and BRD inhibitors (trametinib + i-BET151) along with the control vehicle group ( $n=3-5$ for each arm). P-values 
represent pairwise $t$ test comparison between the experimental arm to vehicle treatment. ${ }^{*}=$ $p<0.05 ;{ }^{* *}=p<0.01 ;{ }^{* * *}=p<0.001 ;{ }^{* * * *}=p<0.0001$. Please refer to Figure S6B for all pair-wise comparisons of the p-values.

I. Tumor volume curves for C1138, F3053, F3008, and B8131 on treatment with MEK inhibitor (binimetinib), BRD inhibitor (i-BET151), and a combination of MEK and BRD inhibitors (binimetinib + iBET151) along with the control vehicle group ( $n=3-5$ for each arm). P-values represent pairwise $t$ test comparison between the experimental arm to vehicle treatment. ${ }^{*}=$ $p<0.05 ;{ }^{* *}=p<0.01 ;{ }^{* * *}=p<0.001 ;{ }^{* * *}=p<0.0001$. Please refer to Figure S6B for all pair-wise comparisons of the p-values.

J. IGV images showing enrichment of H3K27ac peaks around MAP3K20, MAP3K11, MAPK12, AKT2, IRS2, and FGF3 genes using aggregate ChIP-seq profiles of normal colon, adenoma and CRC tumors 
Fig. 3. Reclassification of colorectal cancer tumors based on their global enhancer distribution leads to identification of enhancer-based subtypes.

A. Non-negative matrix factorization (NMF) clustering of CRC adenocarcinoma $(n=33)$ and adenoma $(n=4)$ samples identified four EPIgenome-based Classification (EpiC) clusters of $\mathrm{CRC}$, shown in the color-coded matrix on the top panel. Consensus molecular subtype (CMS) classification of each tumor sample is identified by using a 498 gene-based random forest approach and is overlaid with the EpiC clusters. Clinical data corresponding to the tumor samples in each cluster, including tumor stage, site, pathological grade, race, and gender of the patients, are provided in the lower panels.

B. Table showing enriched major signaling pathways for genes targeted by EpiC-specific enhancers using GREAT(17). False Discovery Rate (FDR) values are color-coded.

C. IGV images showing enrichment of H3K27ac peaks around CTLA4 for EpiC1, TGF $\beta R 3$ in EpiC2, FGF19 in EpiC3, and NPTX2 in EpiC4 using aggregate ChIP-seq profiles of EpiC1, EpiC2, EpiC3 and EpiC4 group tumors.

D. KDE (Kernel Density Estimate) plot with peak lengths (x-axis) and densities (y-axis) of EpiC3 and EpiC3-specific enhancers. Peaks with length more than $5 \mathrm{kbs}$ were annotated as Broad whereas those with $<3 \mathrm{~kb}$ in length were called as Sharp.

E. Pathway analysis for genes in Clusters 2 and 3 from NMF clustering (see Figure S9) of 115 CMS2 TCGA CRC tumors that overlap with EpiC3 and EpiC4-unique genes (LogFC >0.5 and $p$-value < 0.05). Based on the pathway enrichment, Cluster 2 was annotated as "EpiC4-like" whereas Cluster 3 was annotated as "EpiC3-like".

F. Kaplan-Meier plot of survival of CMS2 samples in the TCGA, comparing EpiC4-like patients ( $n=37$ ) and EpiC3-like patients $(n=63)$. The log-rank (Mantel-Cox) p-value is shown for the difference in survival.

G. Motif enrichment analysis for enhancers unique to different EpiC groups using HOMER (25). 
Fig. 4. Correlation between CRC molecular subtypes and EpiCs can predict combinatorial treatments with high clinical significance.

A-C. Summary of $\mathrm{IC}_{50}$ values for 19 small molecule inhibitors (Y-axis) in cell lines with similar expression features to CMS1/EPIC1 (HCT116 or SW480) (A), CMS2/EPIC3 or EPIC4 (T84) (B), and CMS4/EPIC2 (SW620) (C). - $\log \left(I_{50}\right)$ values are demonstrated on X-axis for each of the compounds. Drugs with higher $-\log \left(\mathrm{IC}_{50}\right)$ are highlighted and were further used in combinatorial experiments.

D-F. Growth curves showing responses of CMS-specific CRC cell lines for the drug combinations identified based on the $\mathrm{IC}_{50}$ and AUC indices (panels A-C and Figures S8-S11): CMS1 lines with PARPi (olaparib) + BRDi (iBET-151) (D), CMS2 with EGFRi (gefitinib) + BRDi (RVX-208) (E), and CMS4 with TGF $\beta$ i (SB431542) + BRDi (ISOX-DUAL) (F).

G-I. Tumor volume curves for xenografts in NUDE mice generated from transplantation of EpiC1/CMS1 (G), EpiC3-4/CMS2 (H), and EpiC2/CMS4 (I) cell lines upon treatment with inhibitors of EGFR (gefitinib, 100mg/kg), PARP (olaparib, 50mg/kg), TGF- $\beta$ (SB431542, 10mg/kg), BRDi (i$B E T 151,15 \mathrm{mg} / \mathrm{kg}$ ), or the combination of EGFRi + BRDi, the combination of PARPi + BRDi, and the combination of TGF- $\beta i+B R D i$ along with the control vehicle group. Mice were treated every other day. Best treatment response was observed in the PARPi + BRDi combination in EpiC1/CMS1, EGFRi + BRDi combination in EpiC3-4/CMS2, and TGF- $\beta i+B R D i$ combination in EpiC2/CMS4 lines. * shows $p$-values $<0.05$, and ** shows $p$-values $<0.01$. 
Figure 1

A

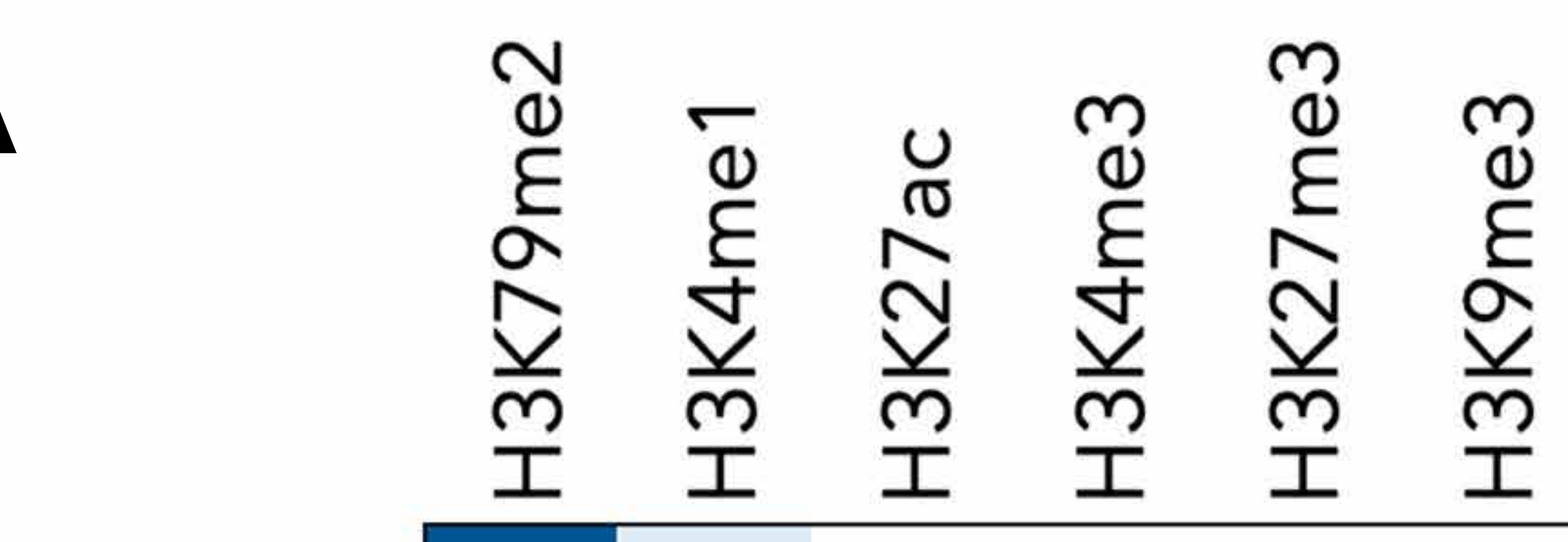

C

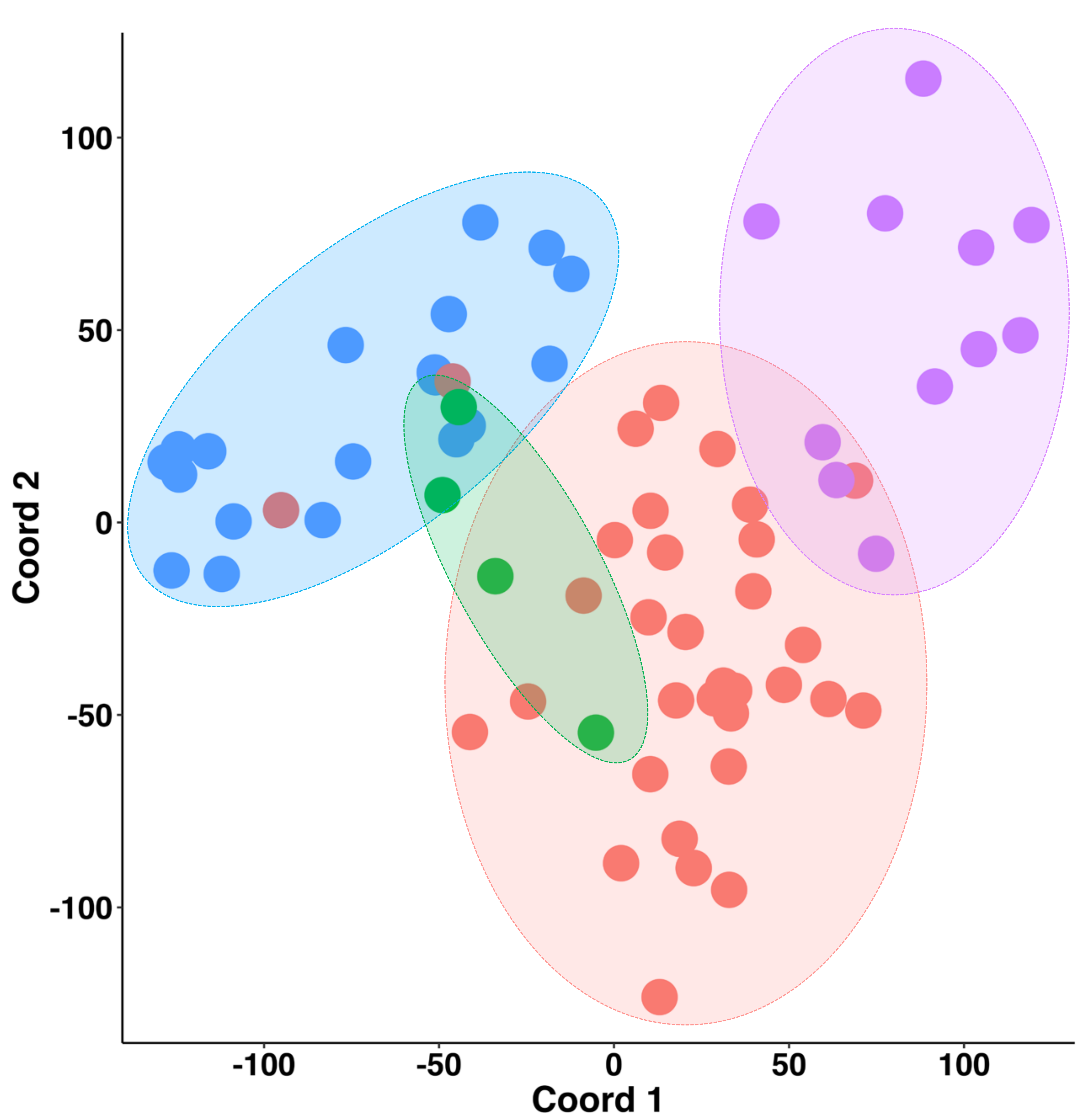

$\mathbf{F}$

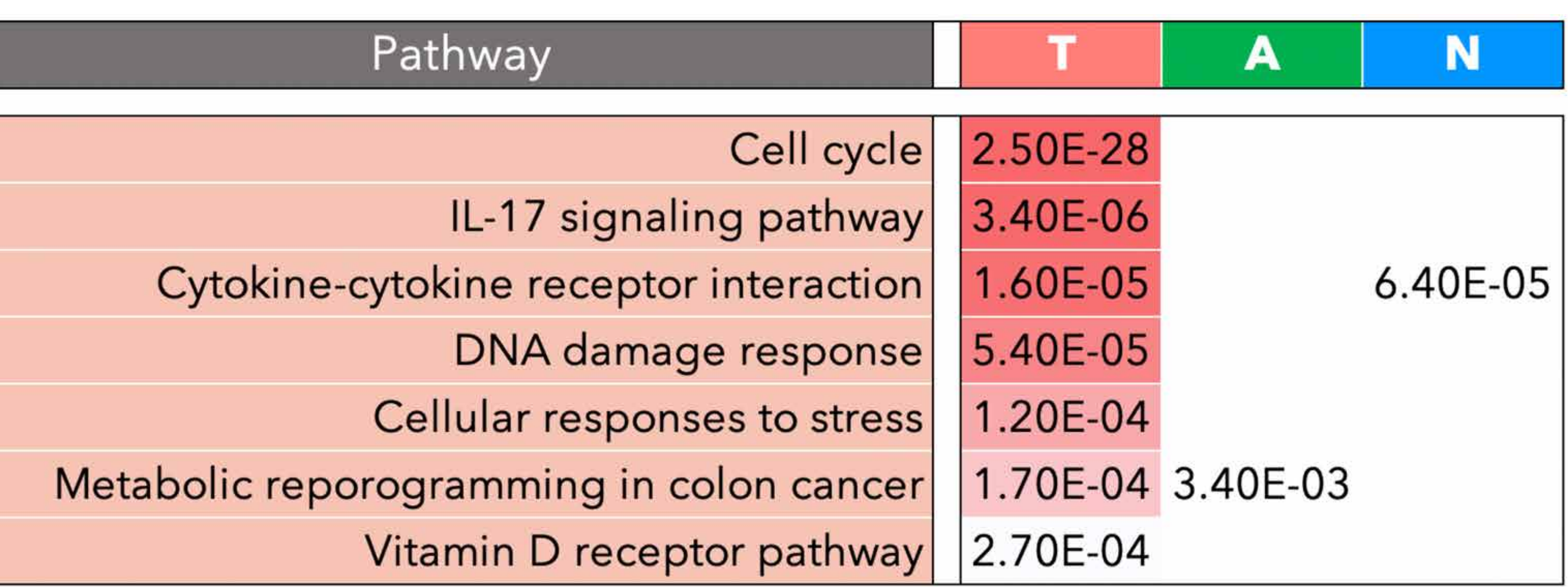

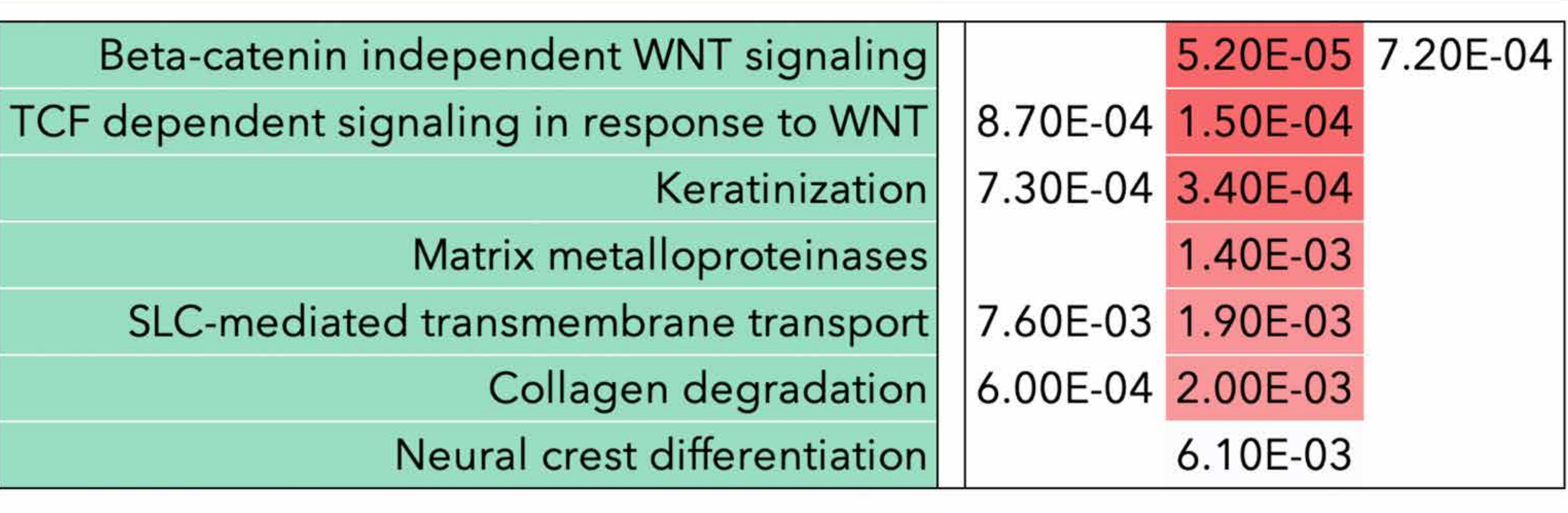

GPCR signaling - G alpha independent $\mid$ 3.80E-05 Glucuronidation Chemokine signaling pathway Vitamin A (retinol) metabolism Th1 and Th2 cell differentiation Extracellular matrix organization MHC class II antigen presentation $\mathrm{Ca} 2+$ pathway Metabolism of lipids Focal adhesion

G
Active Transcription

Transcribed Enhancer

Low Signal

Enhancer

Active Enhancer

Poised Promoter

Polycomb Repressed

Low signal

Poised Heterochromatin

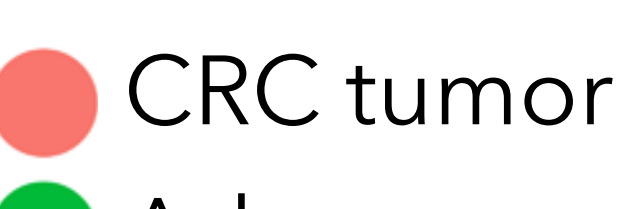

Adenoma

Normal colon
CRC cell lines

1.20E-09

2.30E-07

1.20E-06

$2.30 \mathrm{E}-06$

4.70E-06

$2.80 \mathrm{E}-06$

7.30E-05

$1.00 \mathrm{E}-04$

$1.90 \mathrm{E}-04$

$3.90 \mathrm{E}-04$
Transcribed Promoter

ChlP-seq aggregate tracks

B

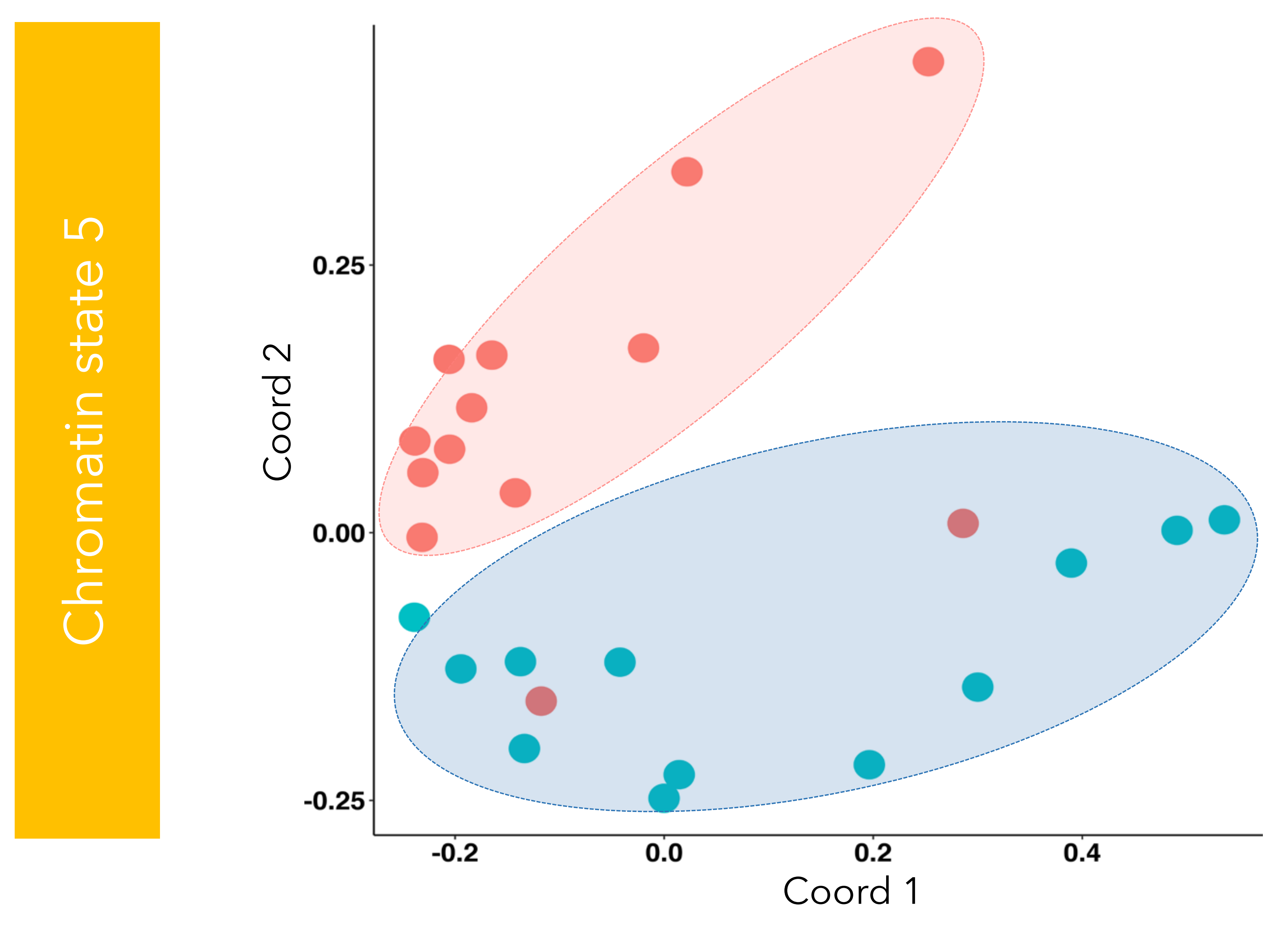

CRC tumo

Normal colon

D

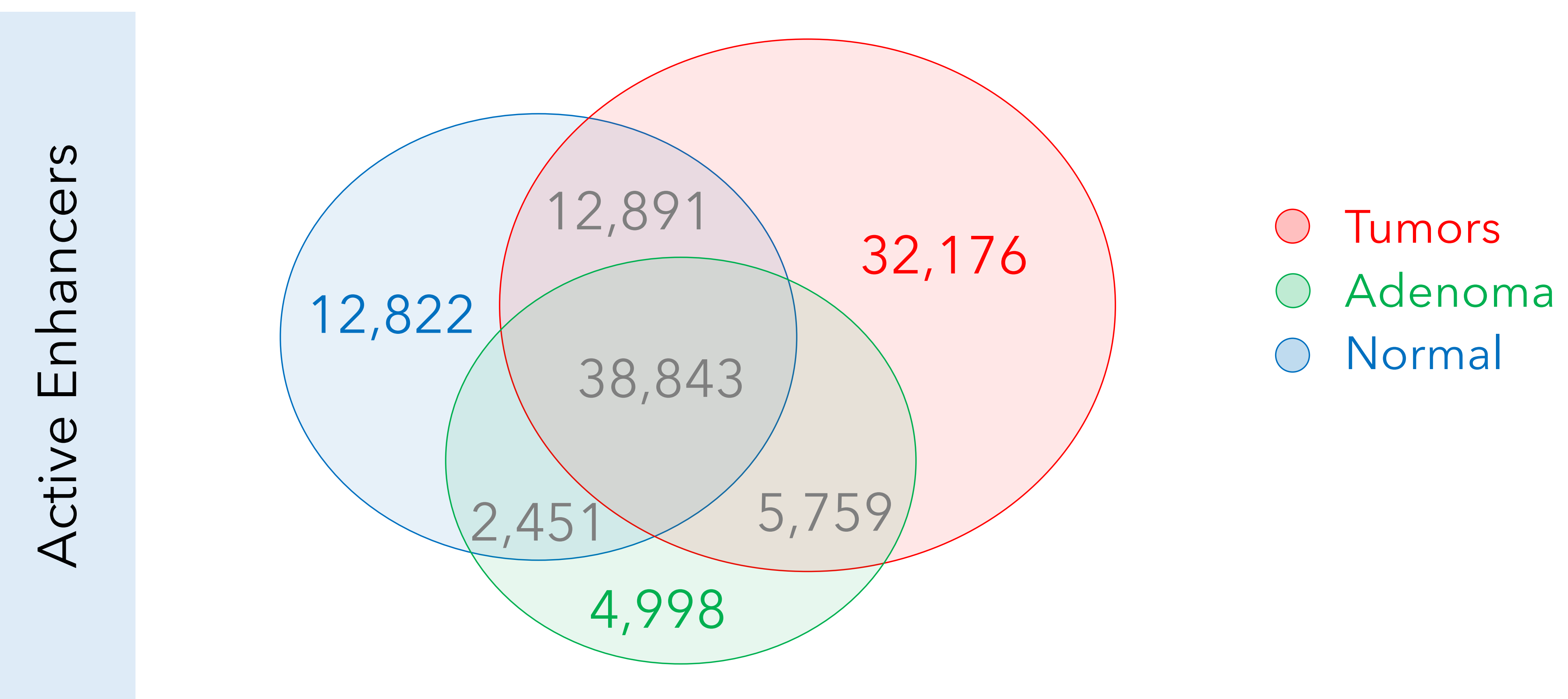

E

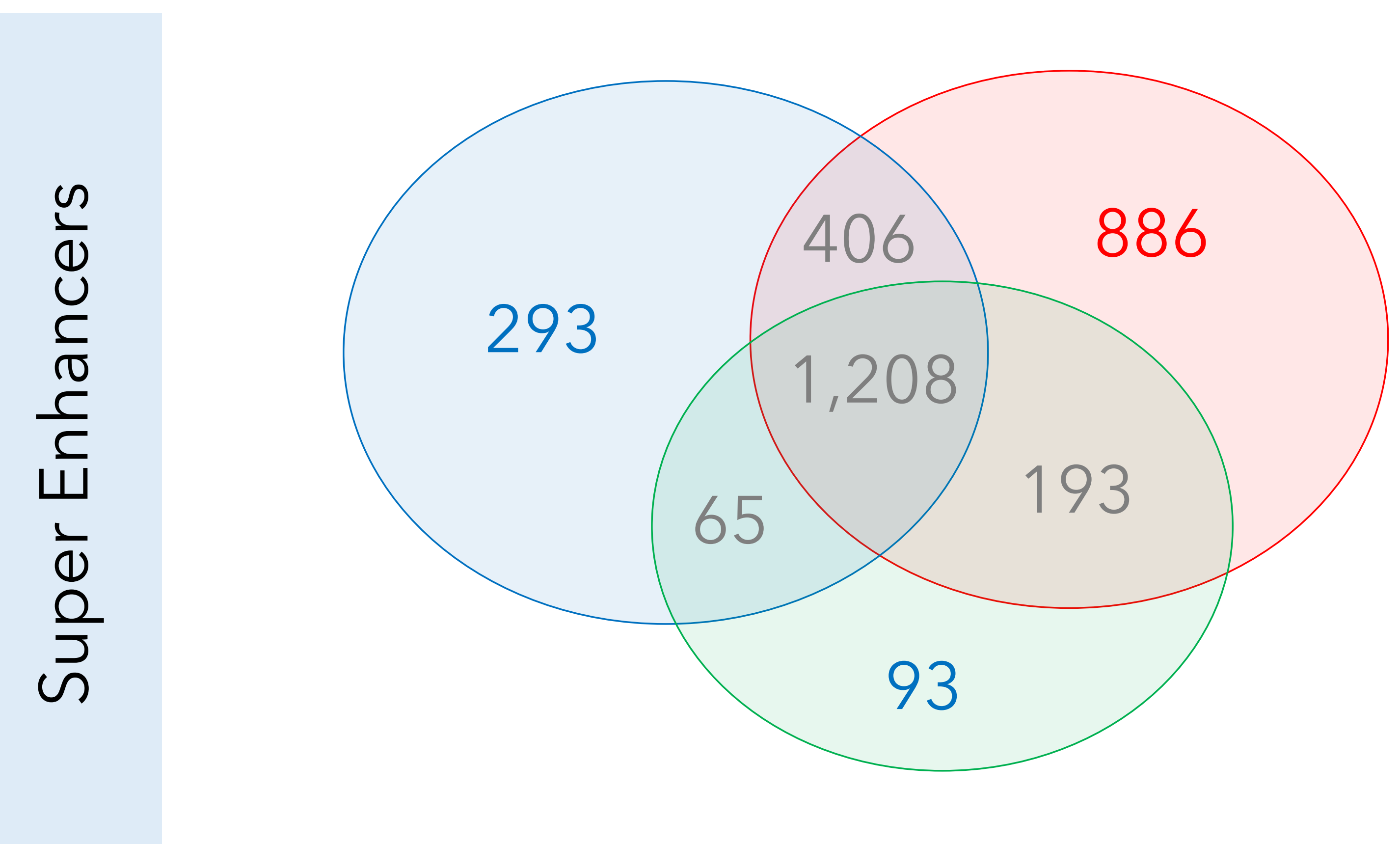

Tumors

- Adenoma

- Normal
H

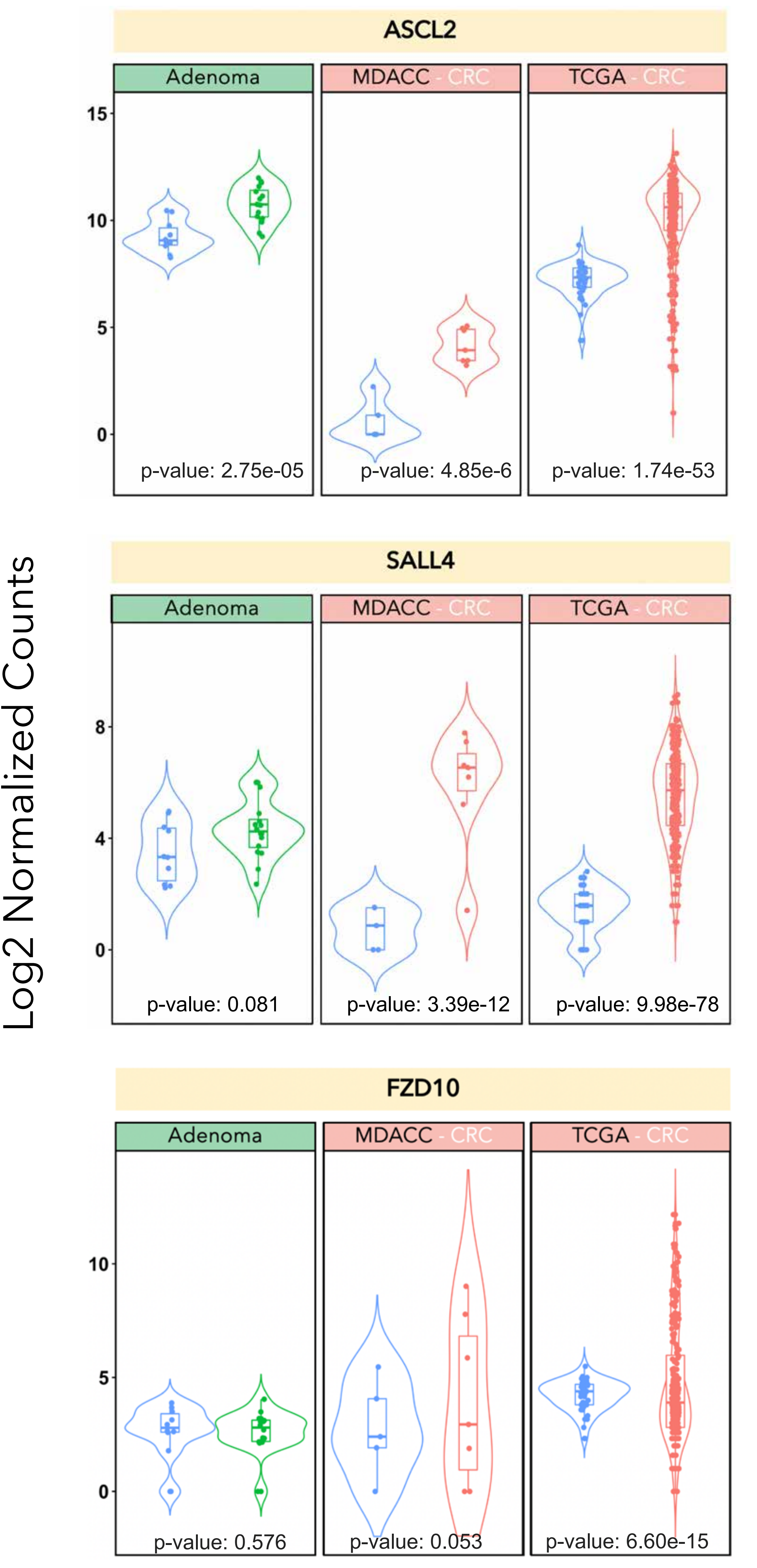

直 Normal 直 CRC 直 Adenoma

\begin{tabular}{|l|l|l|}
\hline \multicolumn{3}{|c|}{ CRC Tumors } \\
\hline \multicolumn{1}{|c|}{ TF Class } & TF & $p$-value \\
\hline \multirow{3}{*}{ Forkhead } & FOXA1 & $1.00 \mathrm{E}-42$ \\
& FOXA2 & $1.00 \mathrm{E}-40$ \\
& FOXM1 & $1.00 \mathrm{E}-33$ \\
\multirow{3}{*}{ ETS } & ETV4 & $1.00 \mathrm{E}-36$ \\
& FLI1 & $1.00 \mathrm{E}-33$ \\
& ERG & $1.00 \mathrm{E}-32$ \\
bZIP & ATF3 & $1.00 \mathrm{E}-36$ \\
& AP-1 & $1.00 \mathrm{E}-33$ \\
\hline
\end{tabular}

\begin{tabular}{|l|l|l|}
\hline \multicolumn{3}{|c|}{ Normal } \\
\hline TF Class & \multicolumn{1}{c|}{ TF } & $p$-value \\
\hline Homeobox & NKX3.1 & $1.00 \mathrm{E}-05$ \\
IRF & IRF2 & $1.00 \mathrm{E}-05$ \\
& IRF1 & $1.00 \mathrm{E}-05$ \\
bHLH & NeuroG2 & $1.00 \mathrm{E}-05$ \\
Forkhead & TCF4 & $1.00 \mathrm{E}-05$ \\
& FOXO1 & $1.00 \mathrm{E}-05$ \\
\hline
\end{tabular}

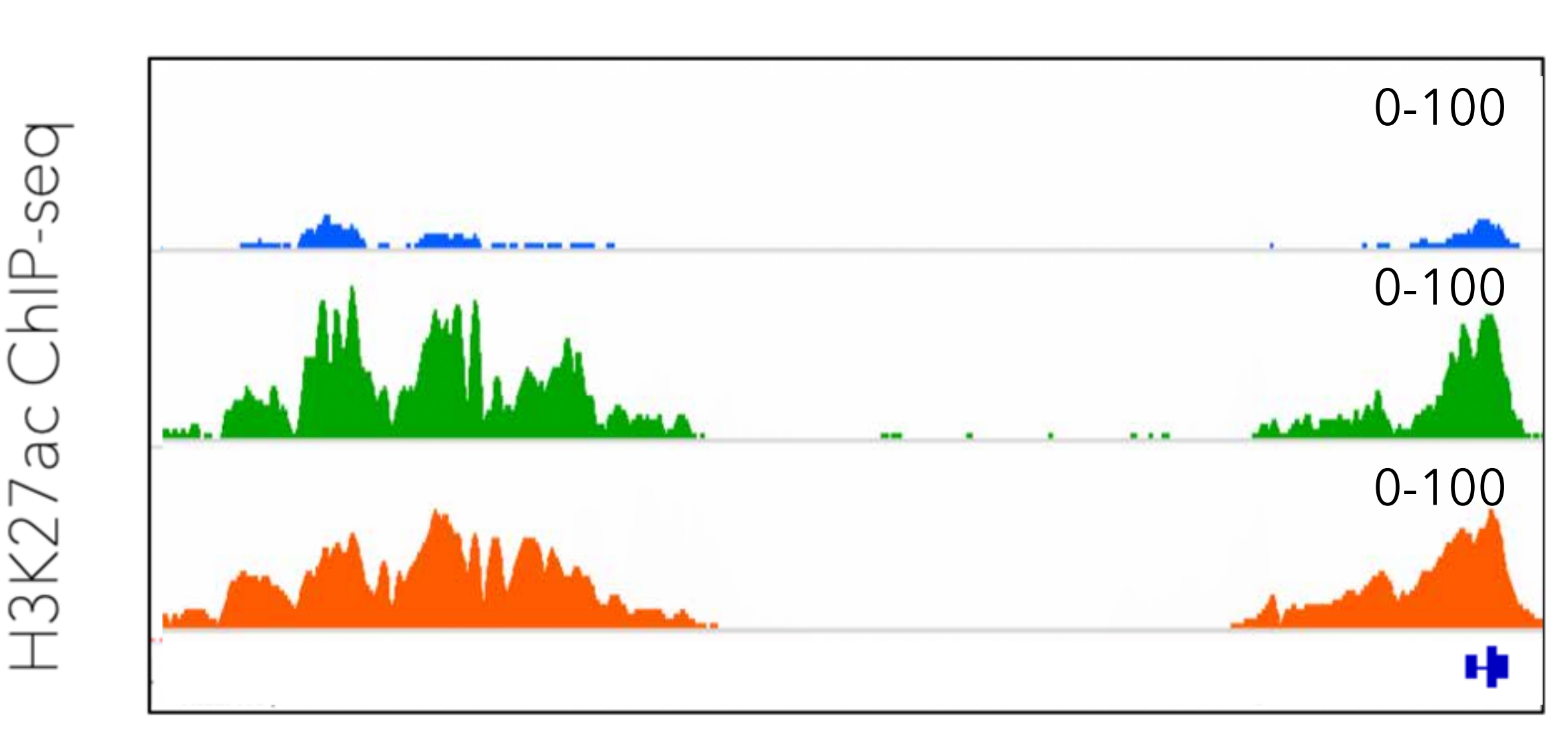

ASCL2

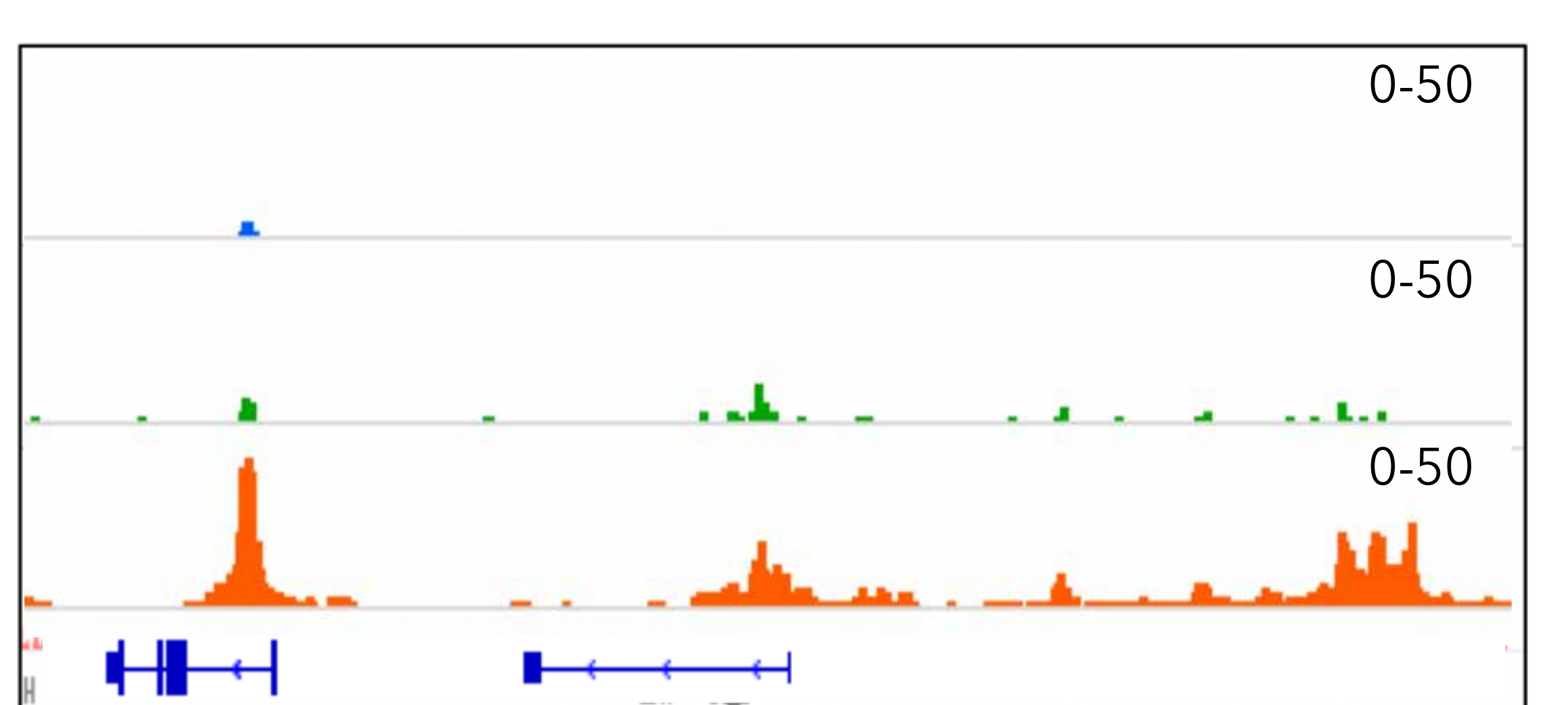

SALL4

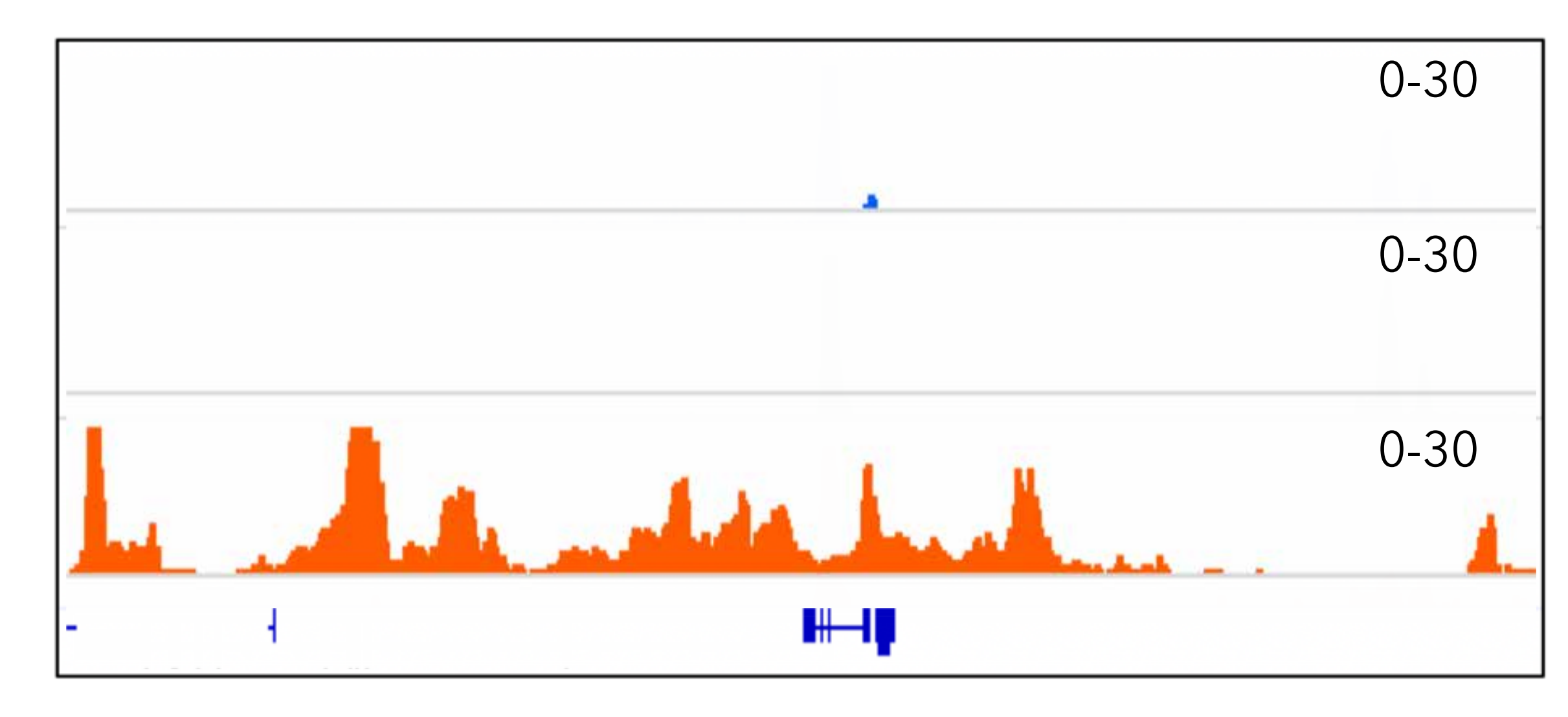

Normal

Adenoma

- CRC Tumors 
Figure 2

A

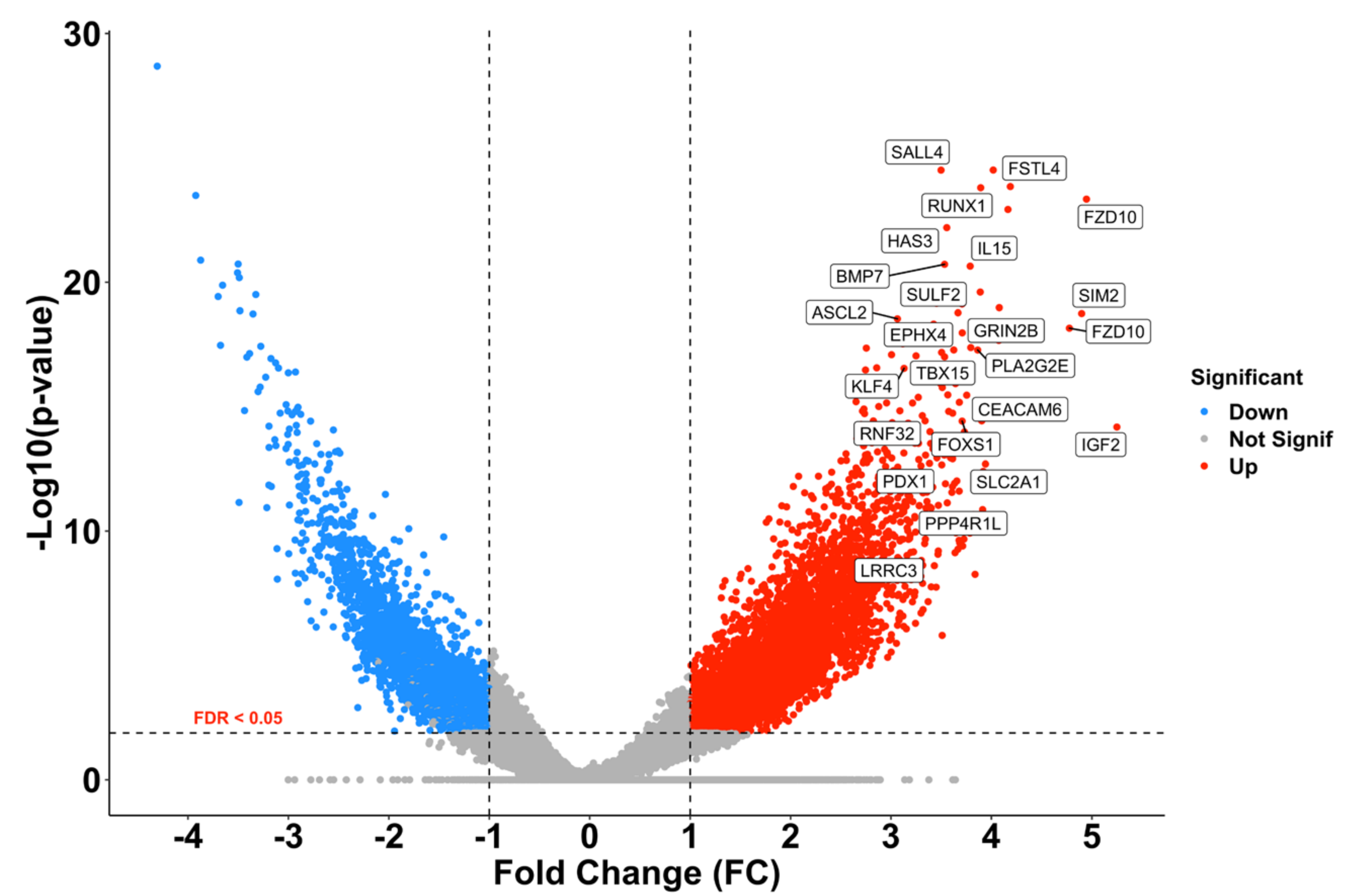

C

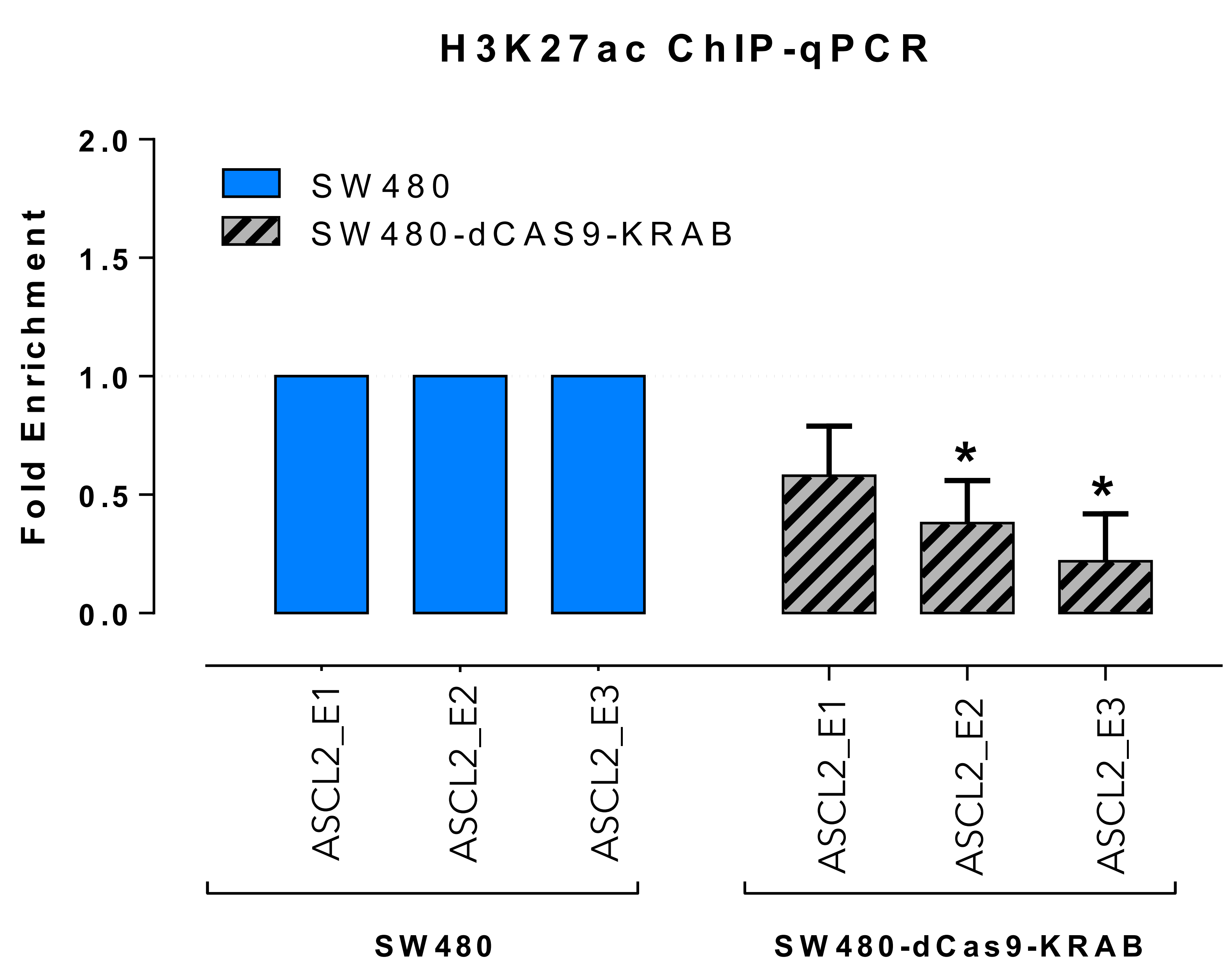

$\mathbf{F}$

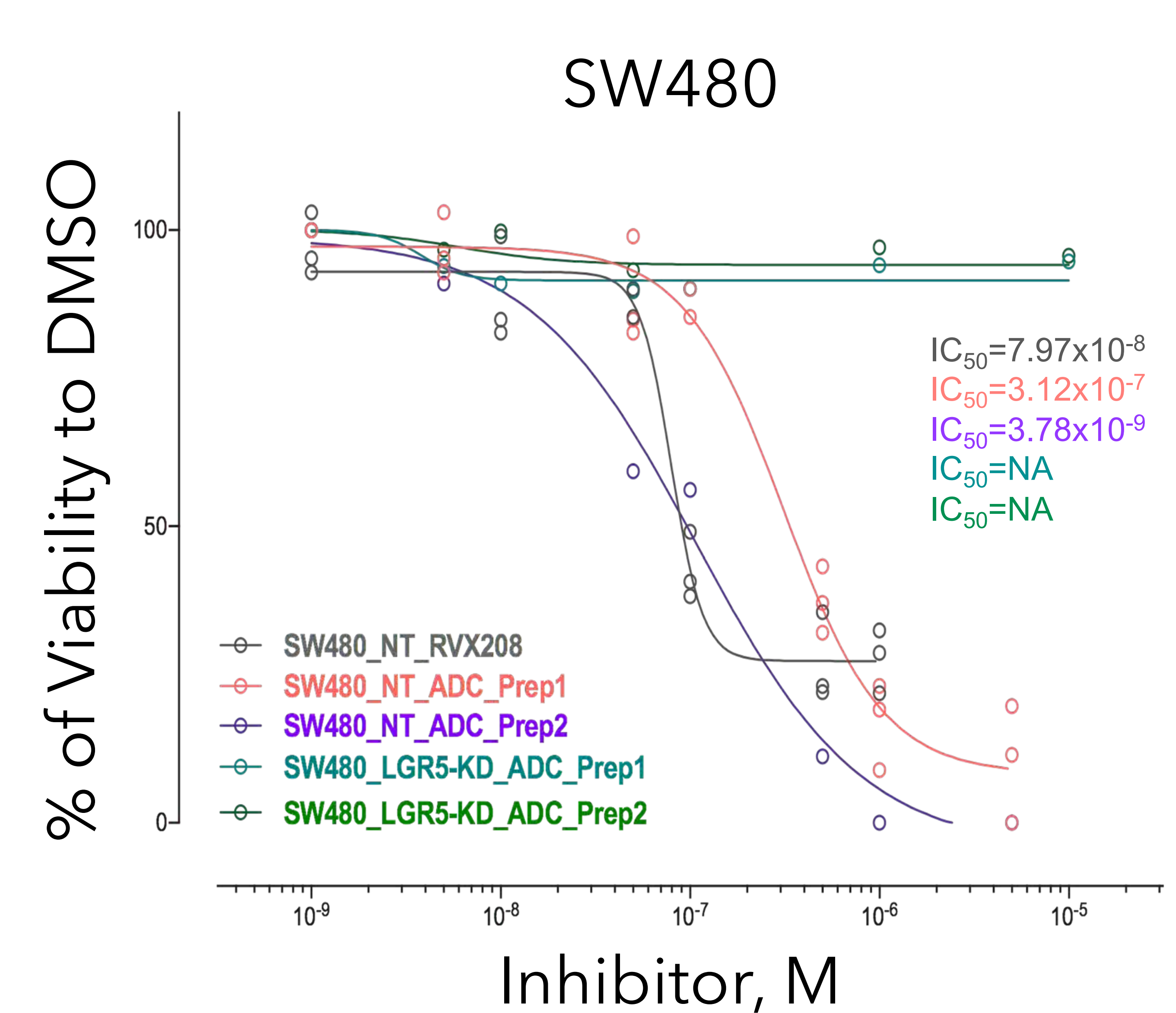

H

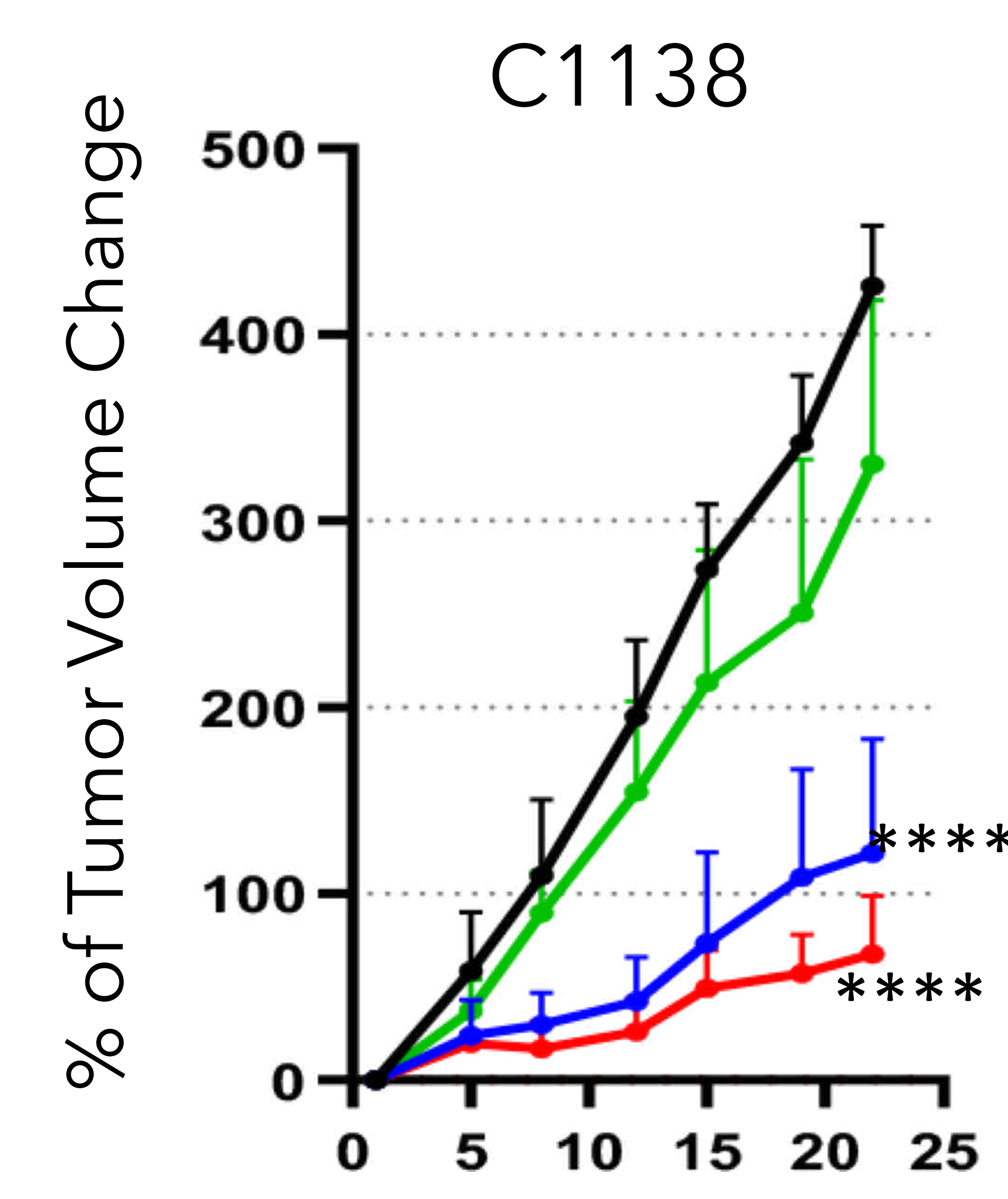

I

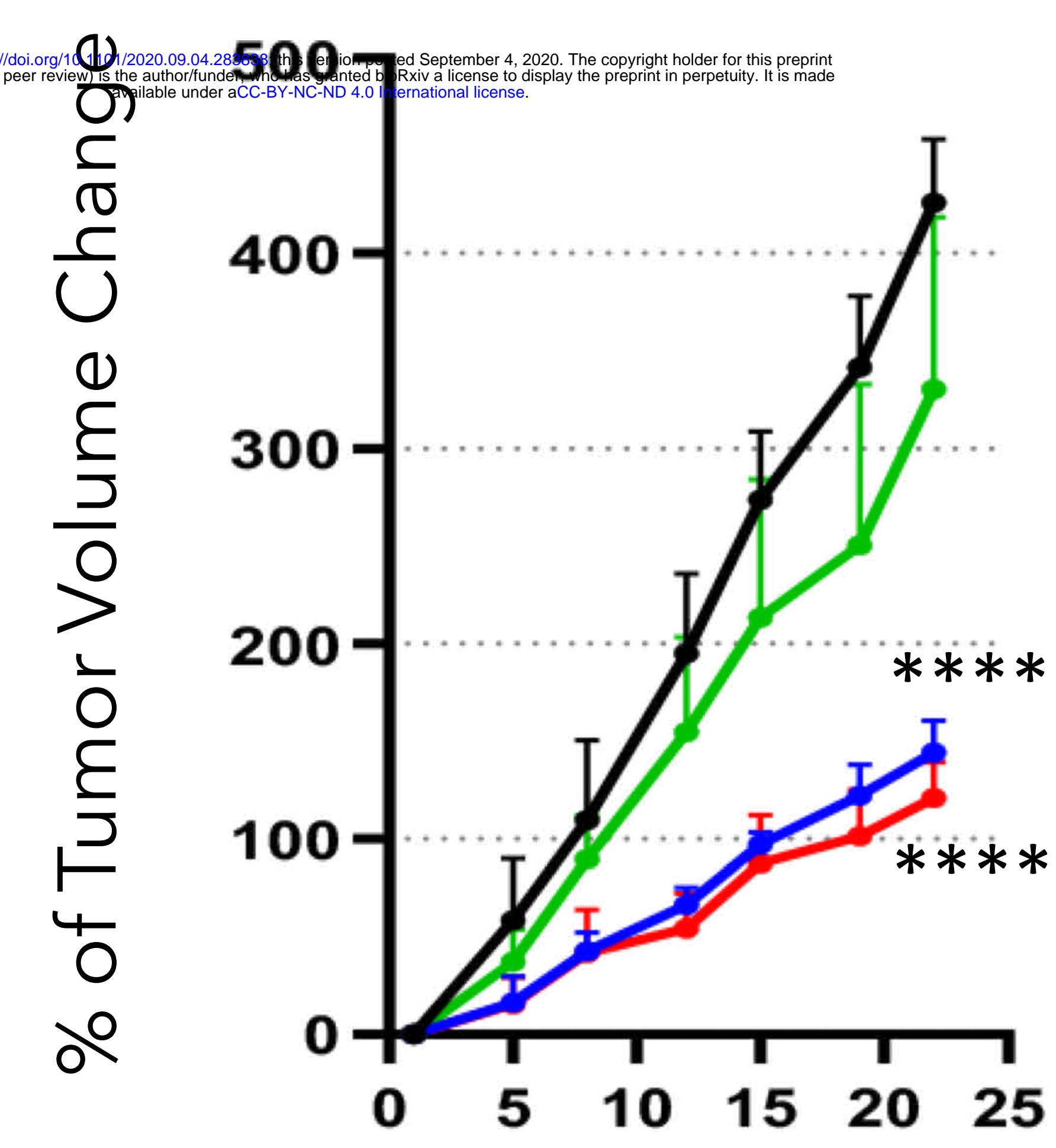

G
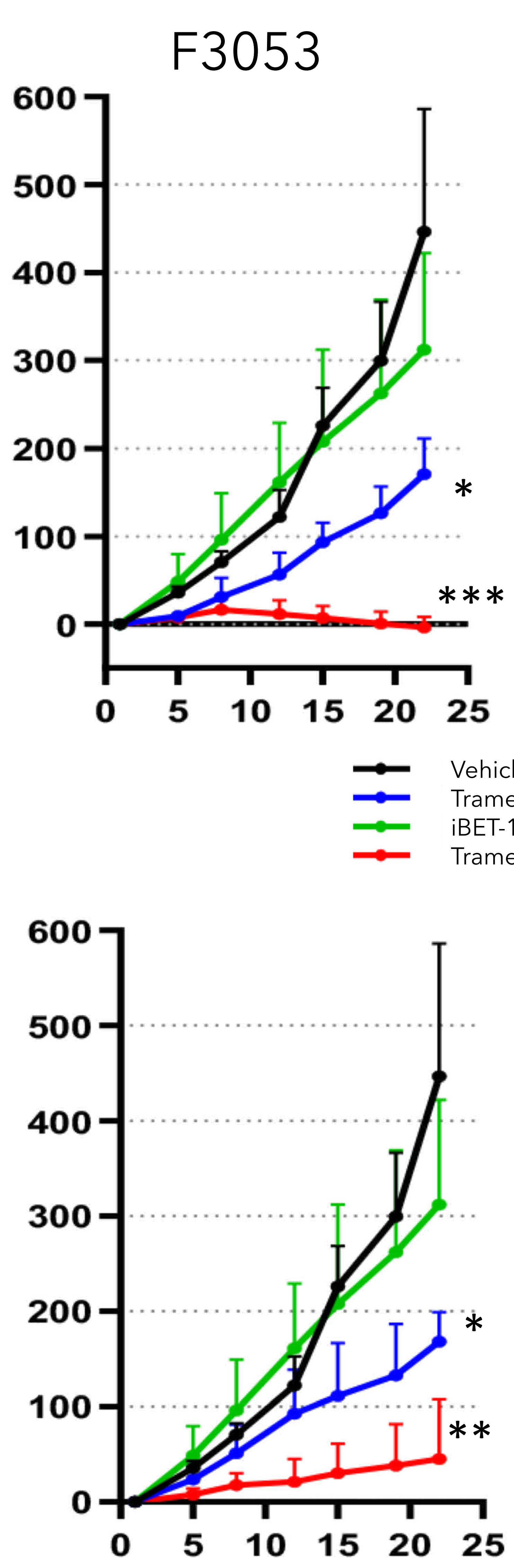

B

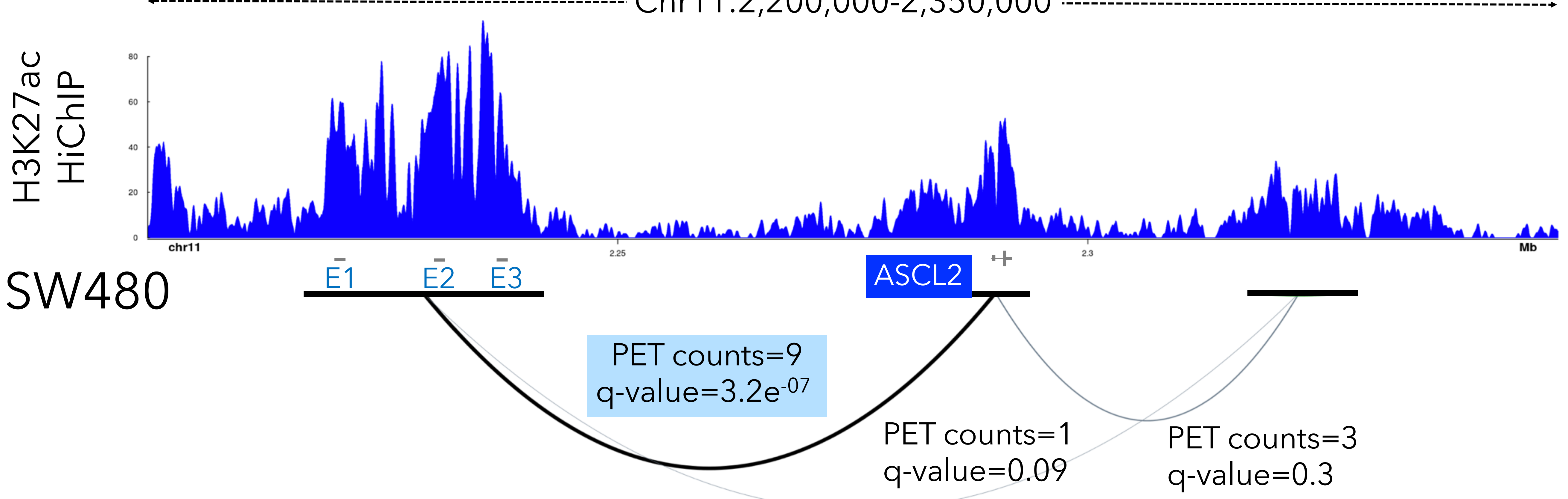

D
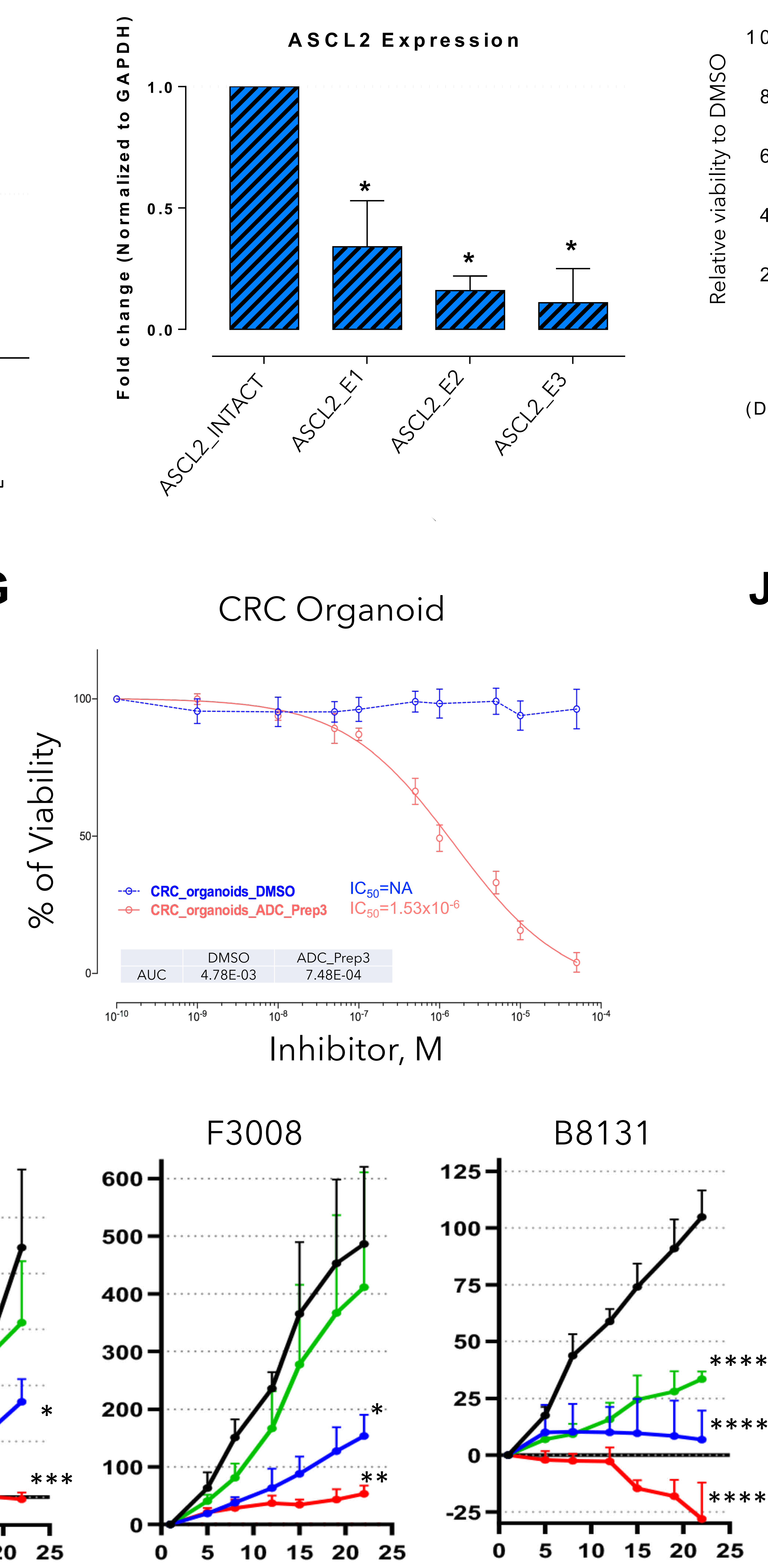

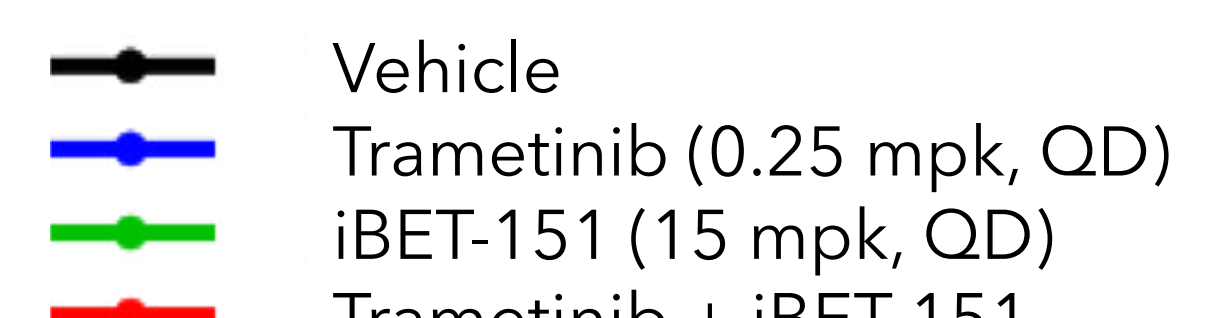

E

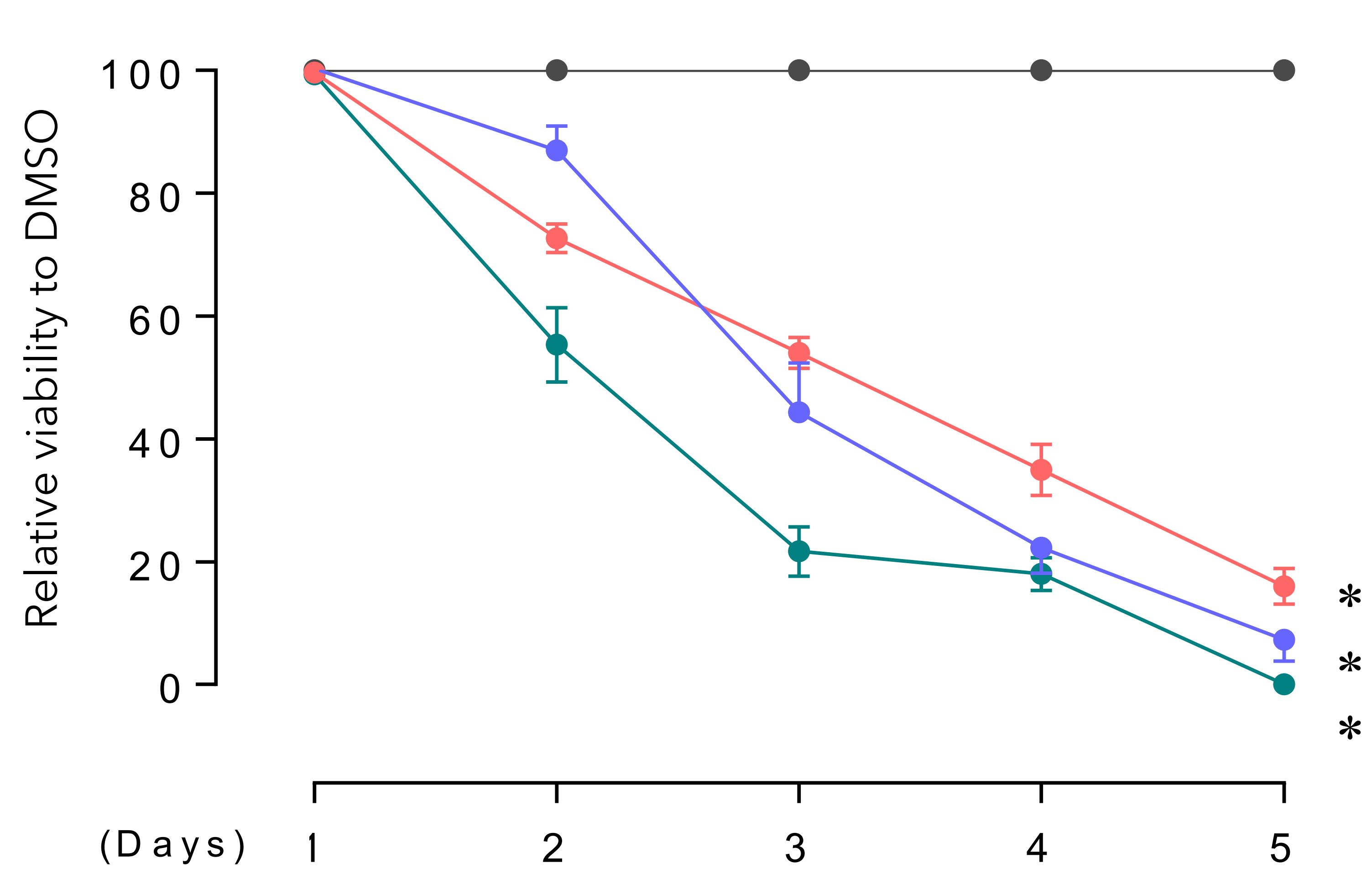

$\longrightarrow$ ASCL2_INTACT - ASCL2_E2

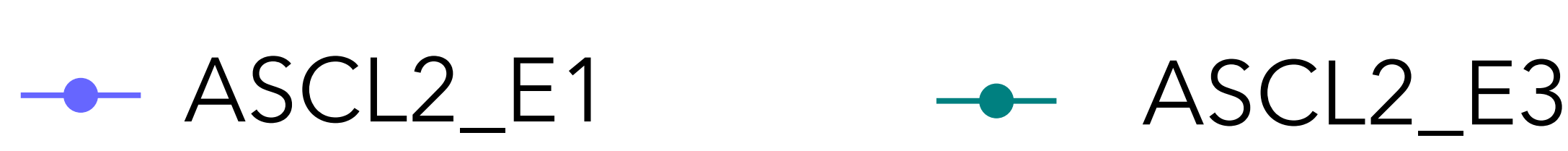

J
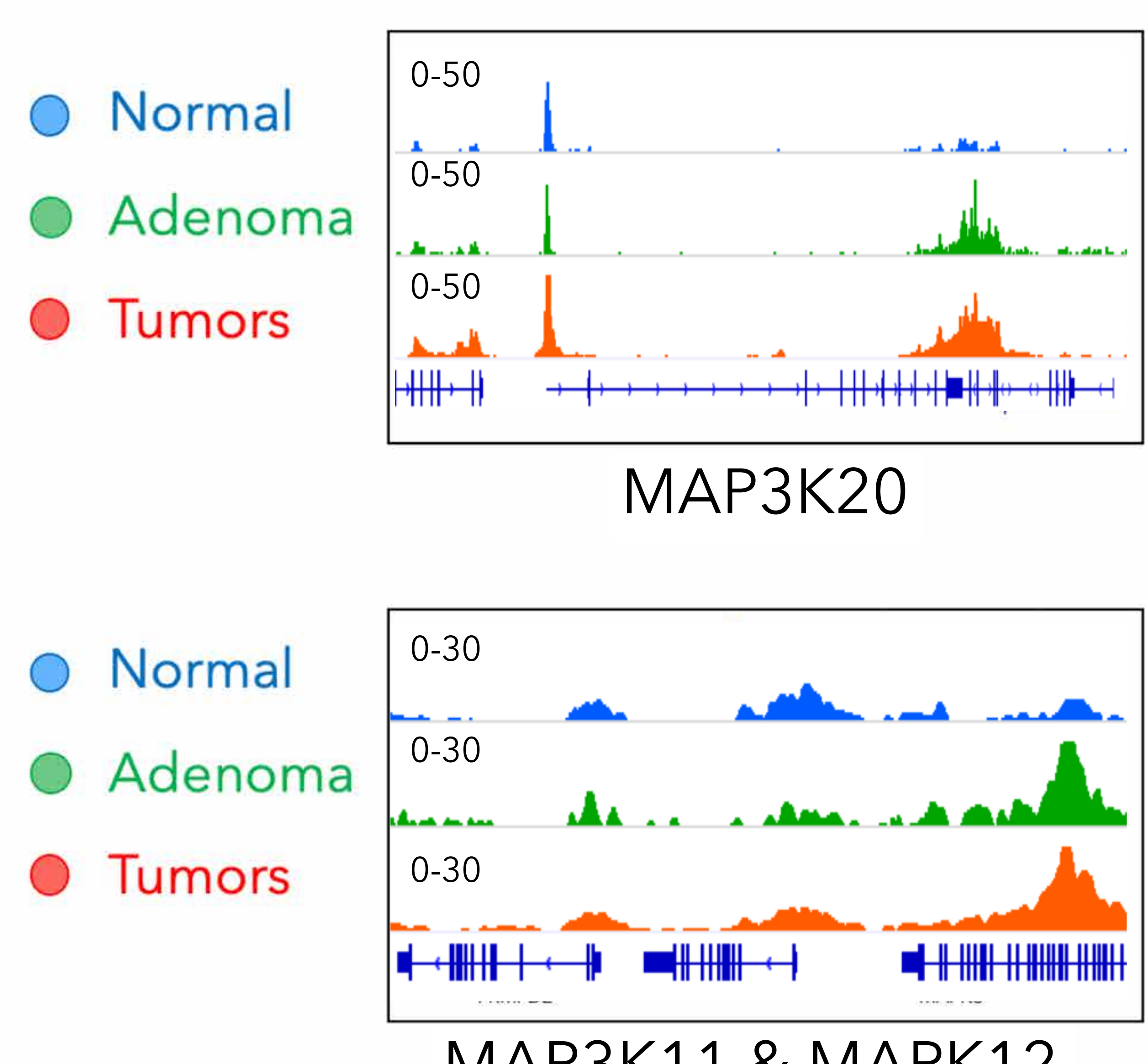

Normal

- Adenoma

- Tumors
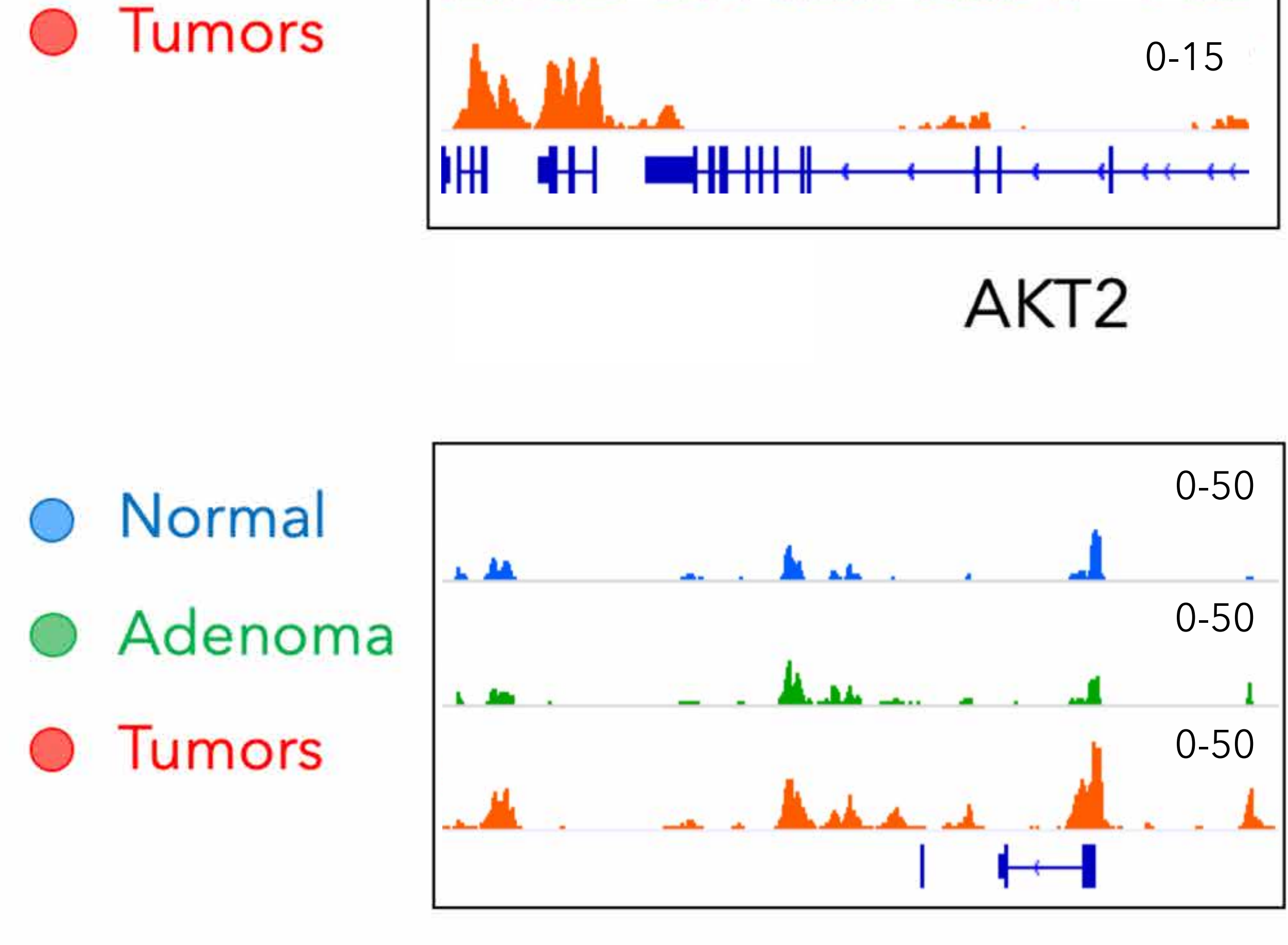

IRS2

Normal

Adenoma

Tumors

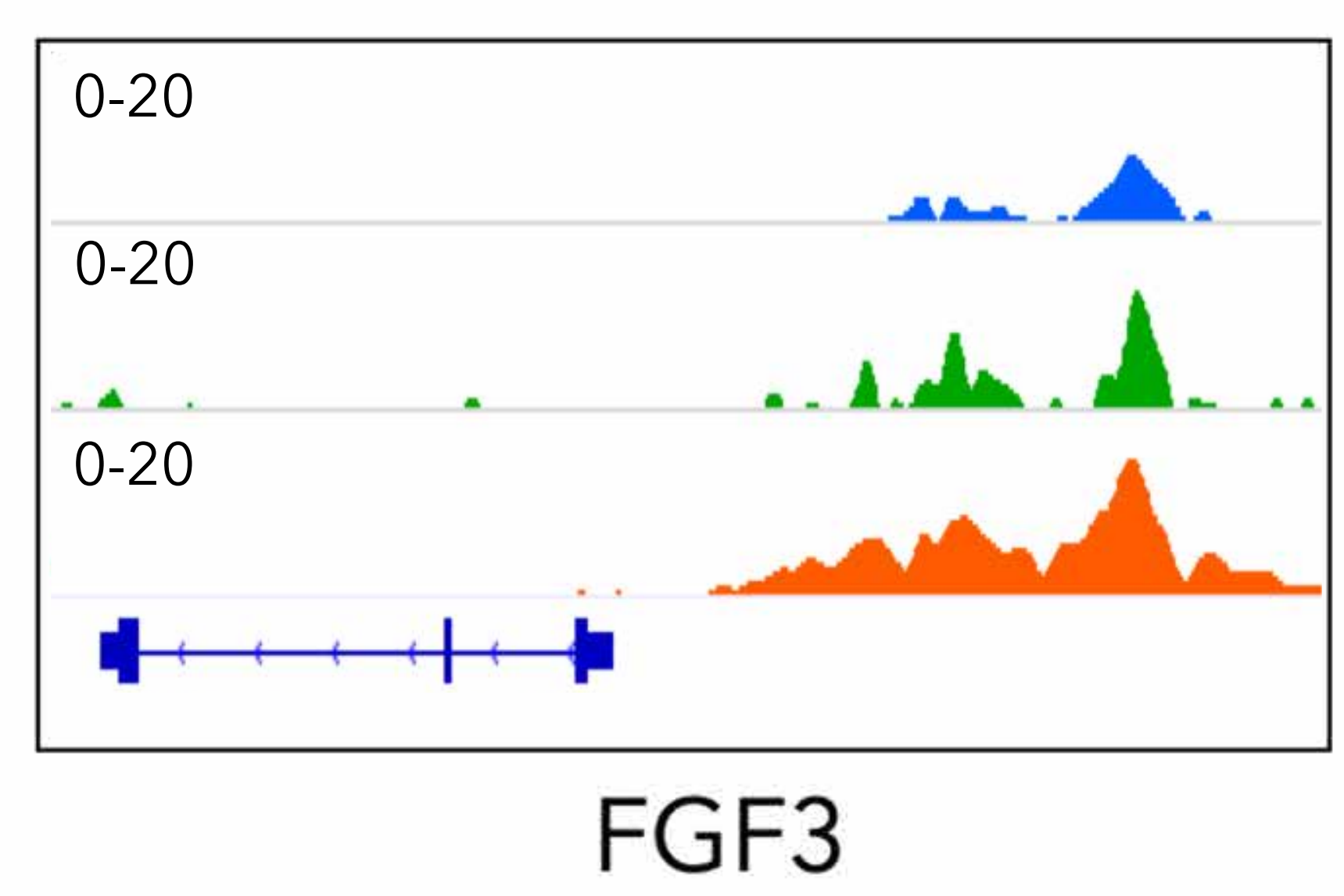




\section{Figure 3}

A

EpiC

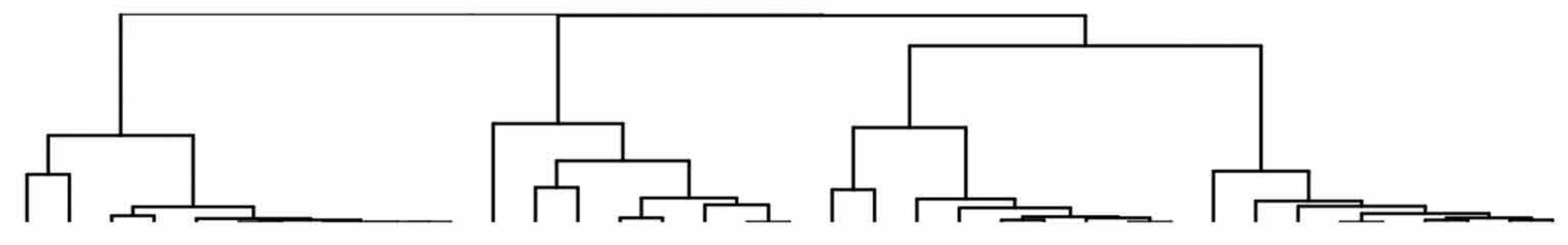

CMS
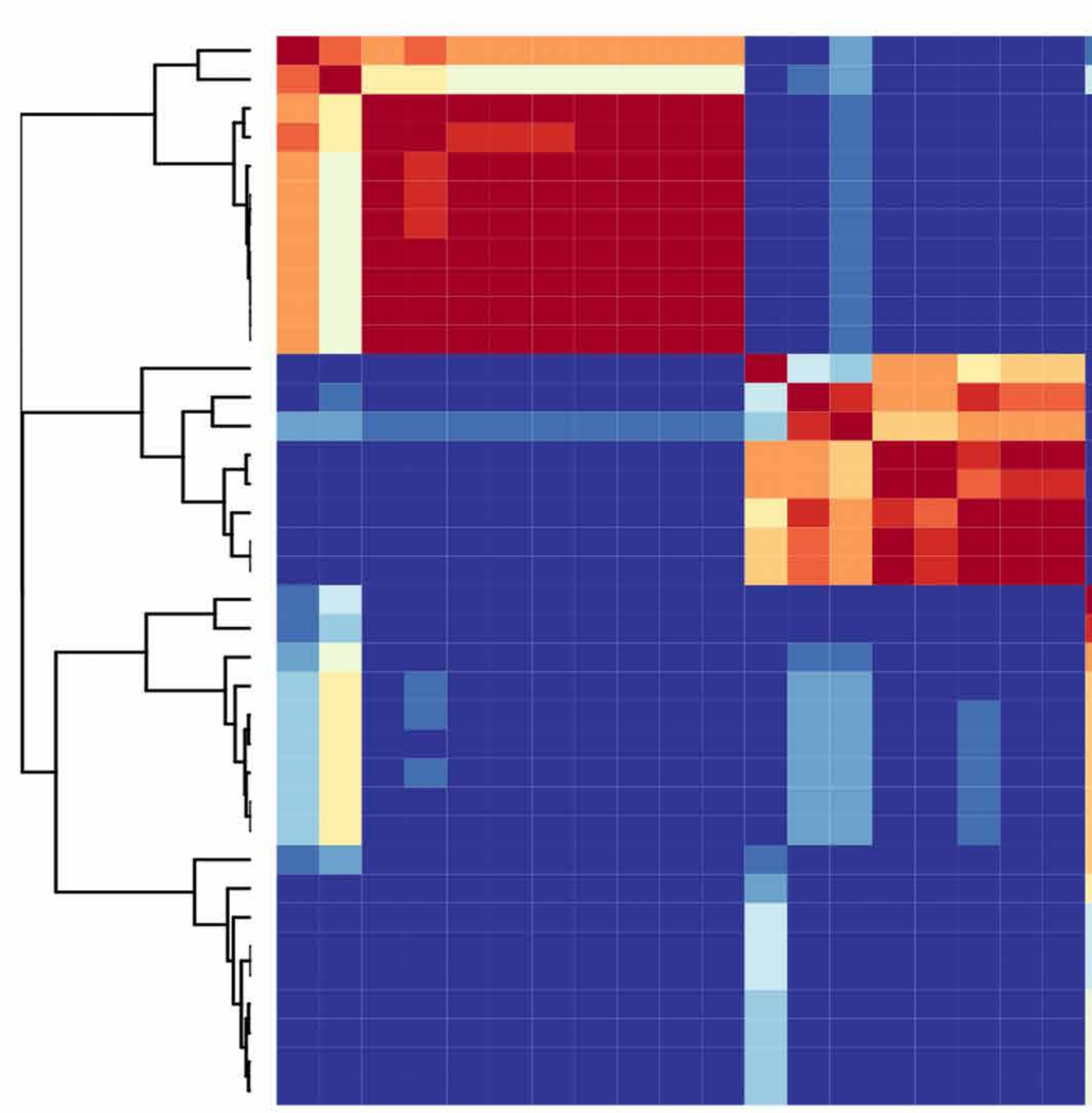

Stage Race

Site

Grade

Gender

Pathology
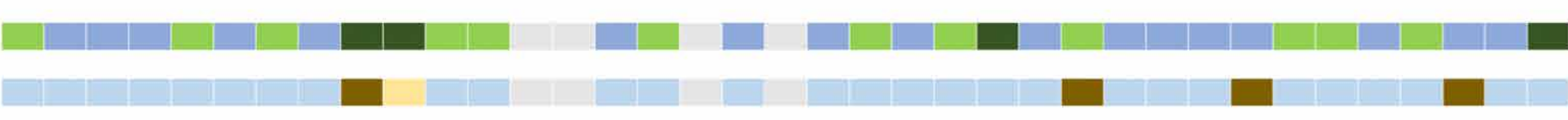

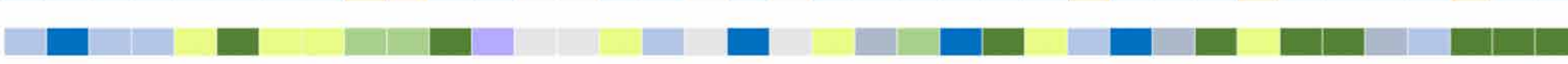
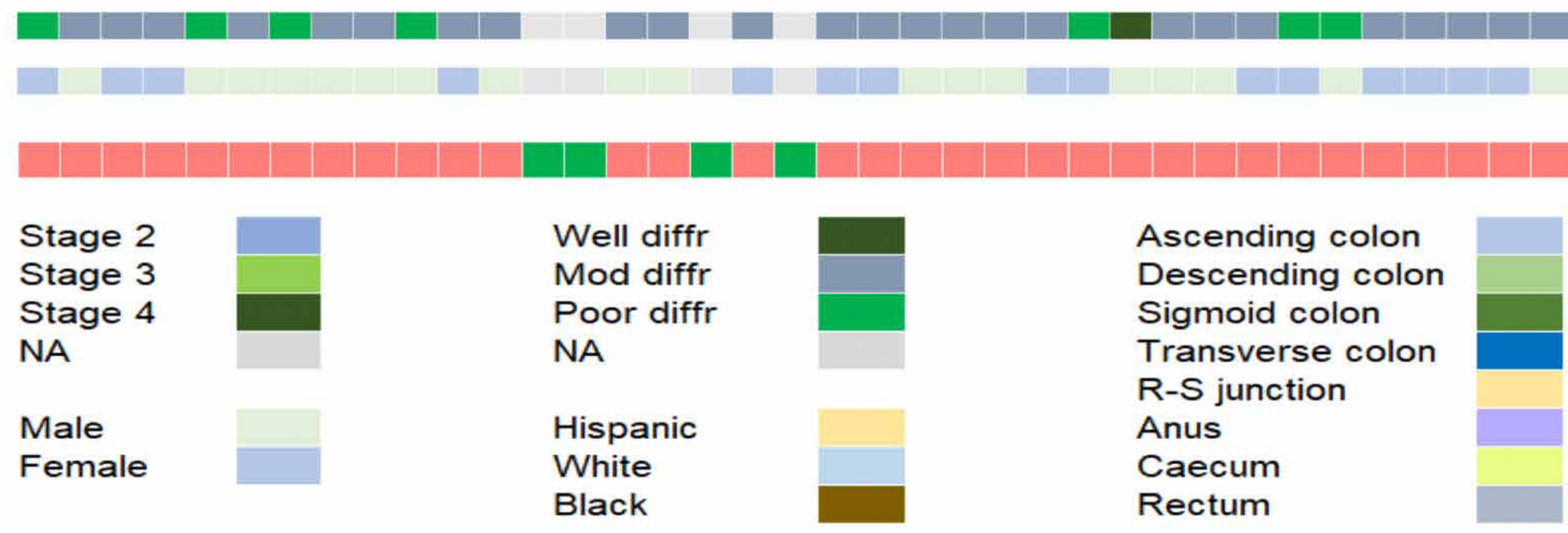

CRC (Adenocarcinoma)
B

\begin{tabular}{|c|c|c|c|c|c|}
\hline & CMS & CMS1 & CMS4 & \multicolumn{2}{|c|}{ CMS2 } \\
\hline & EpiC & EpiC1 & EpiC2 & EpiC3 & EpiC4 \\
\hline \multicolumn{6}{|l|}{ PATHWAY } \\
\hline TCR signaling & & 4.5巨-07 & & & \\
\hline B cell activation & & 4.7E-06 & & & \\
\hline Immune response & & 1.0E-05 & & & \\
\hline Ca2+ channel & & 2.0€-05 & 1.4€-03 & 9.8E-03 & \\
\hline Inflammation & & 2.0E-04 & & & \\
\hline WNT signaling & & & $2.2 \leftleftarrows-06$ & 1.1E-04 & $2.4 \sqsubset-03$ \\
\hline O-linked glycosylation & & 2.5E-04 & 7.9巨-05 & & \\
\hline TGF $\beta$ pathway & & $2.3 €-03$ & $1.4 €-04$ & $1.5 €-04$ & \\
\hline GPCR signaling & & $7.6 巨-05$ & $1.2 \leftleftarrows-04$ & $1.2 \leftleftarrows-03$ & $2.2 €-04$ \\
\hline VEGF pathway & & $1.8 €-03$ & & $5.0 E-03$ & \\
\hline EGFR signaling & & & $2.0 €-03$ & $3.0 E-06$ & \\
\hline TAp63 signaling & & & & $6.0 \leftleftarrows-05$ & \\
\hline MAPK pathway & & 4.6巨-04 & $3.1 E-03$ & 8.5€-05 & \\
\hline C-MYC targets & & & & 2.0E-04 & \\
\hline Cellular senescence & & & & 5.0E-04 & \\
\hline GPCR Signaling & & & & & 2.0Е-04 \\
\hline IGF signaling & & & & & $6.1 €-04$ \\
\hline PI3K-AKT pathway & & & & & 3.0E-03 \\
\hline Neurotransmitter activity & & & & & 3.3E-03 \\
\hline
\end{tabular}

C

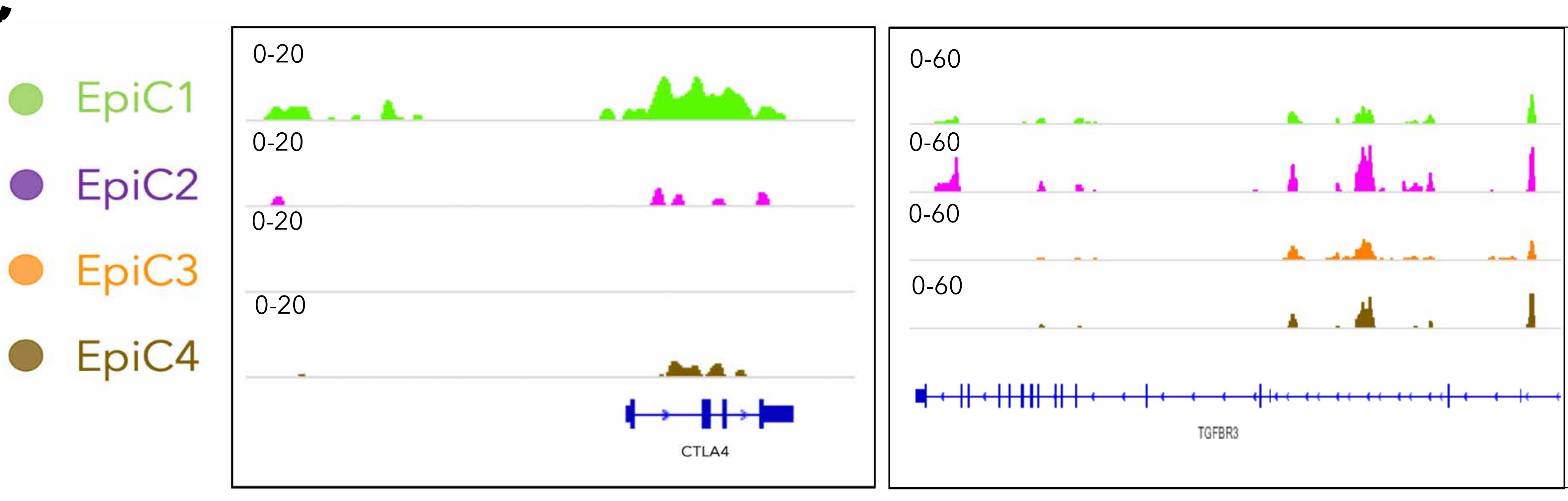

CTLA4

TGFßR3

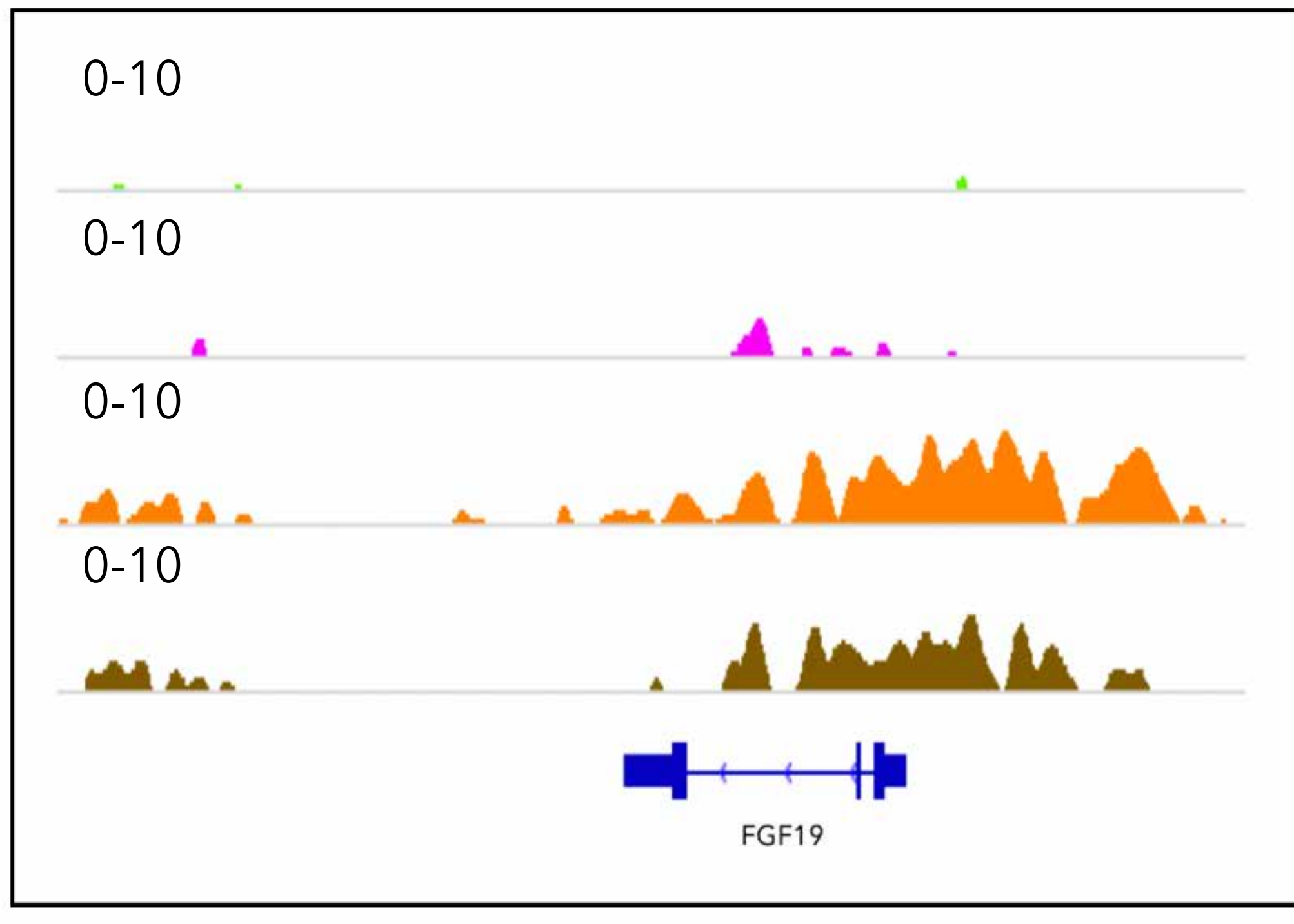

FGF19

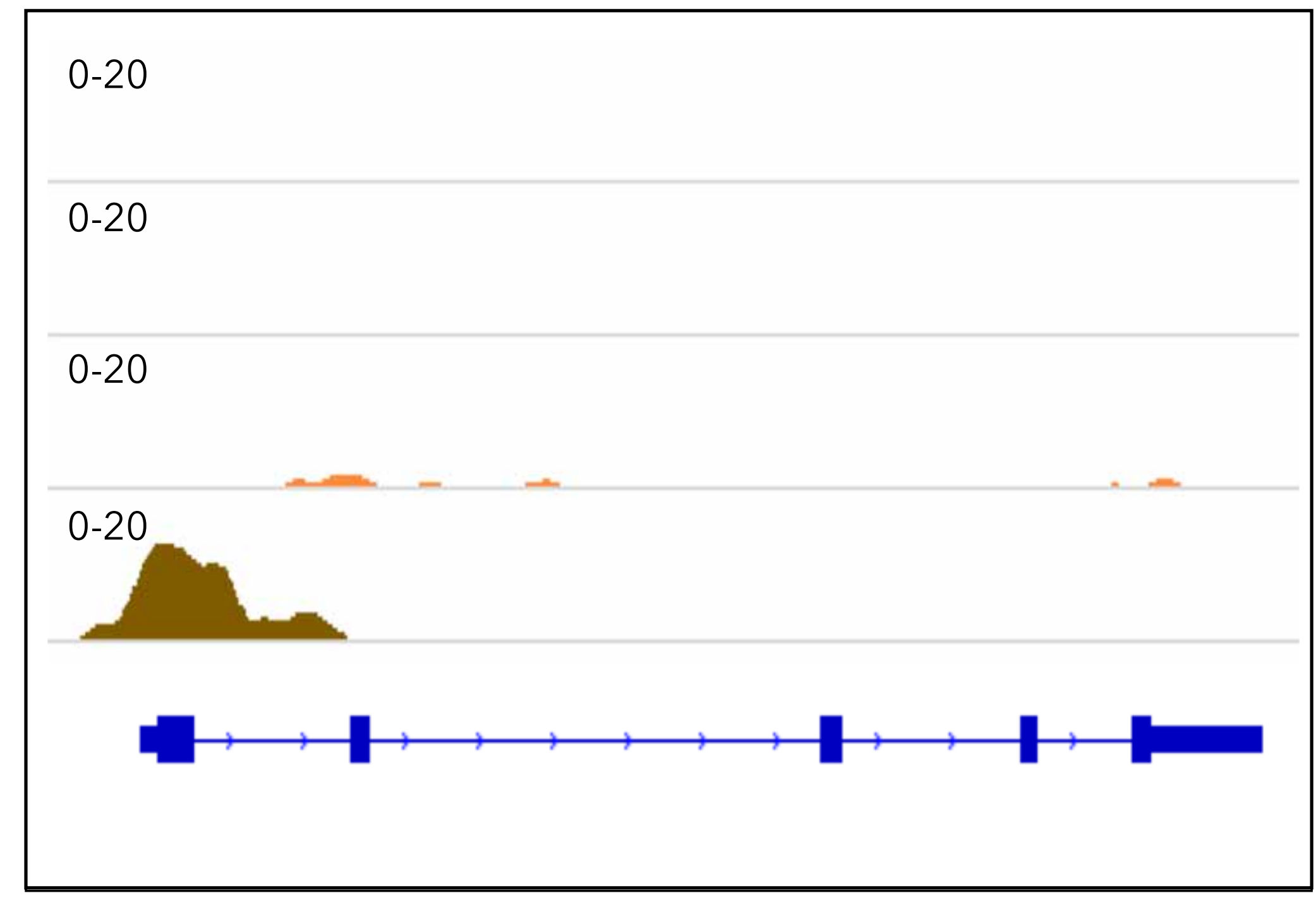

NPTX2
D

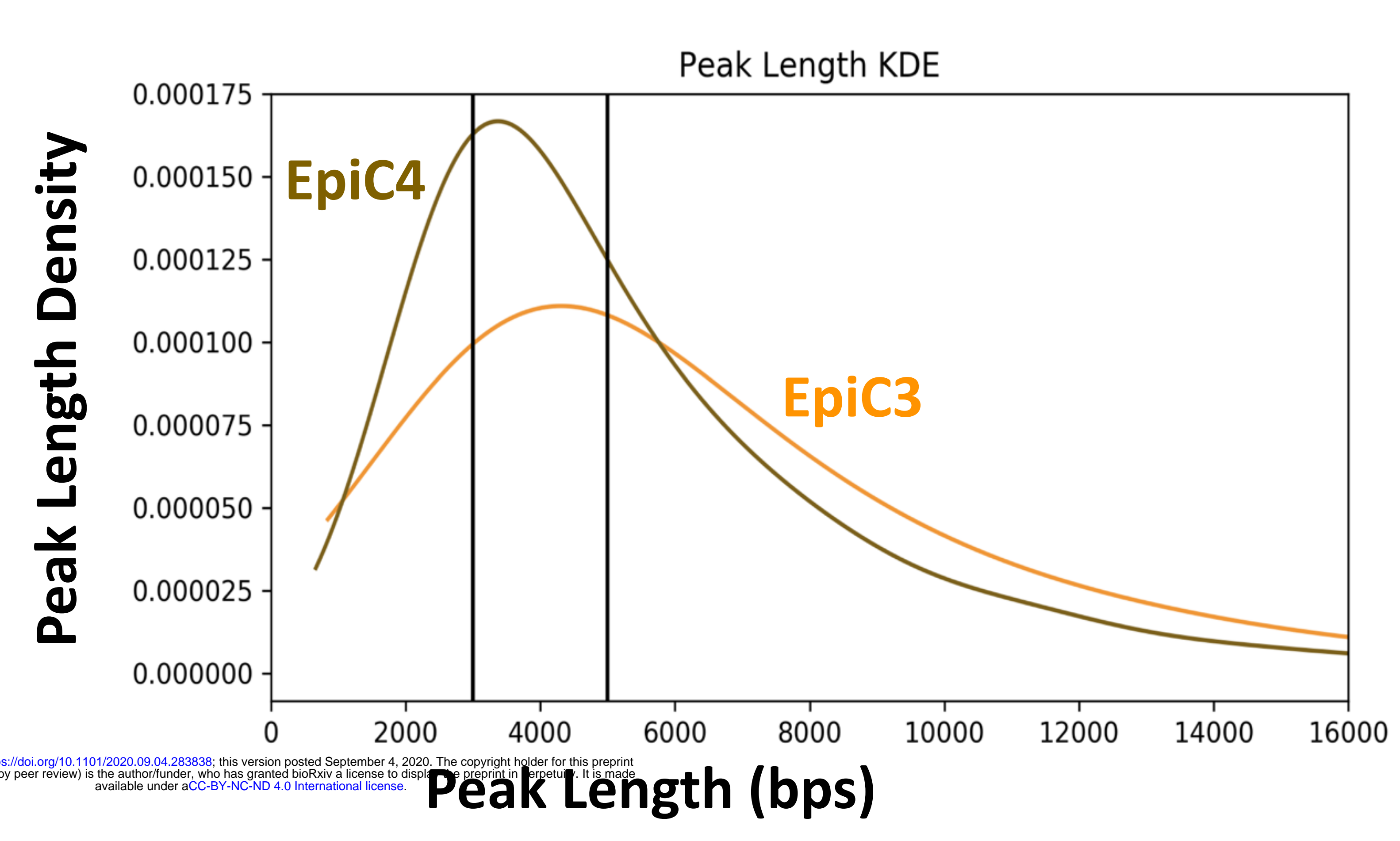

F

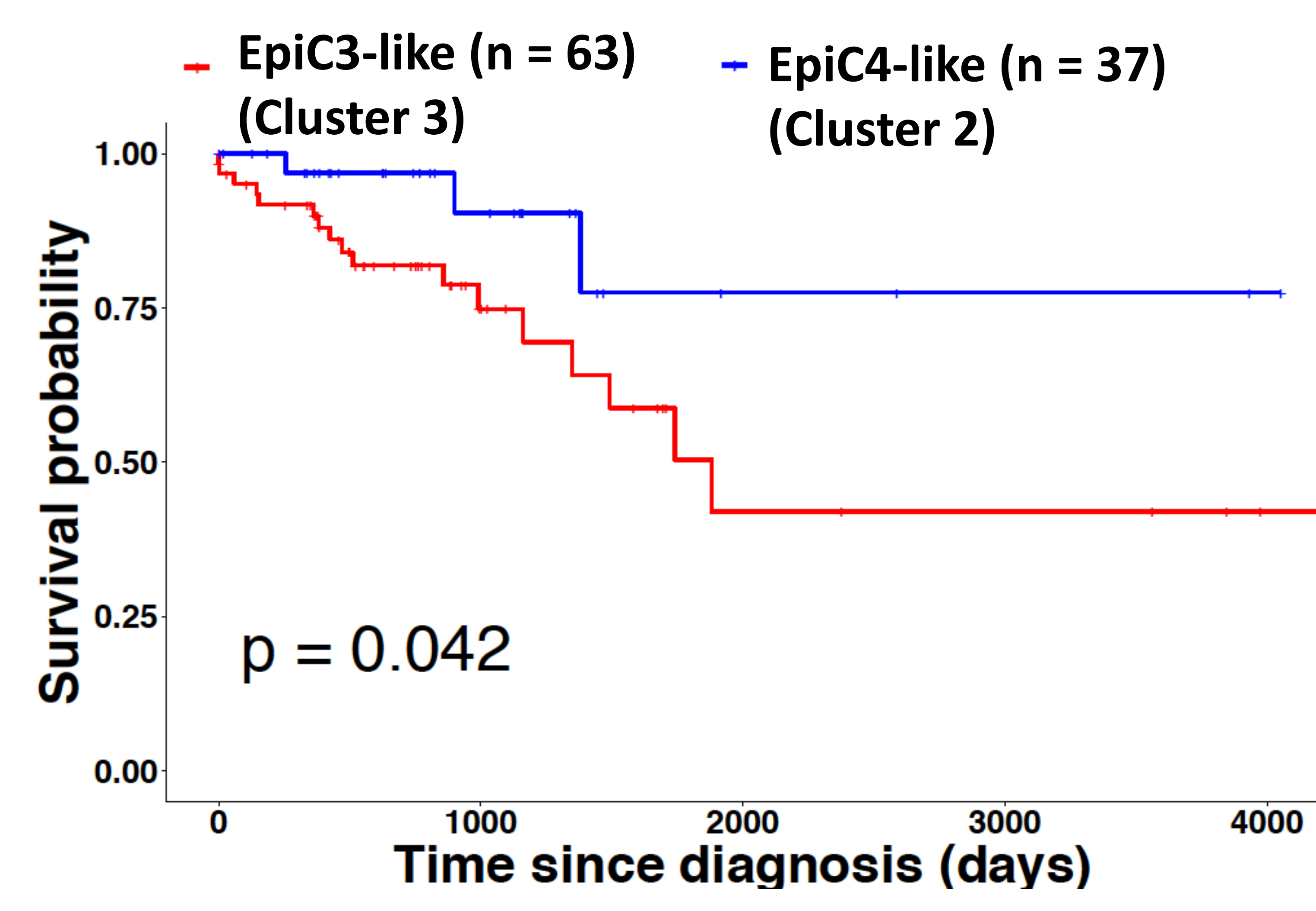

E

\begin{tabular}{|r|r|} 
Epic3-like (Cluster 3) \\
\hline PATHWAY & FDR \\
\hline Matrix mettaloproteinases & $1.52 \mathrm{E}-04$ \\
\hline ErbB signaling & $1.14 \mathrm{E}-03$ \\
\hline MAPK Signaling & $1.36 \mathrm{E}-02$ \\
\hline C-MYC targets & $1.73 \mathrm{E}-02$ \\
\hline EGFR & $2.16 \mathrm{E}-02$ \\
\hline
\end{tabular}

EpiC4-like (Cluster 2) \begin{tabular}{|l|r|}
\hline PATHWAY & FDR \\
\hline
\end{tabular}

\begin{tabular}{|r|r|}
\hline PATHWAY & FDR \\
\hline Epithelial-to-Mesenchymal Transition & $6.04 \mathrm{E}-04$ \\
\hline NCAM1 Interactions & $7.03 \mathrm{E}-04$ \\
\hline PI3K-AKT Pathway & $5.17 \mathrm{E}-03$ \\
\hline Axon guidance & $5.98 \mathrm{E}-03$ \\
\hline GPCR Signaling & $3.14 \mathrm{E}-02$
\end{tabular}

G

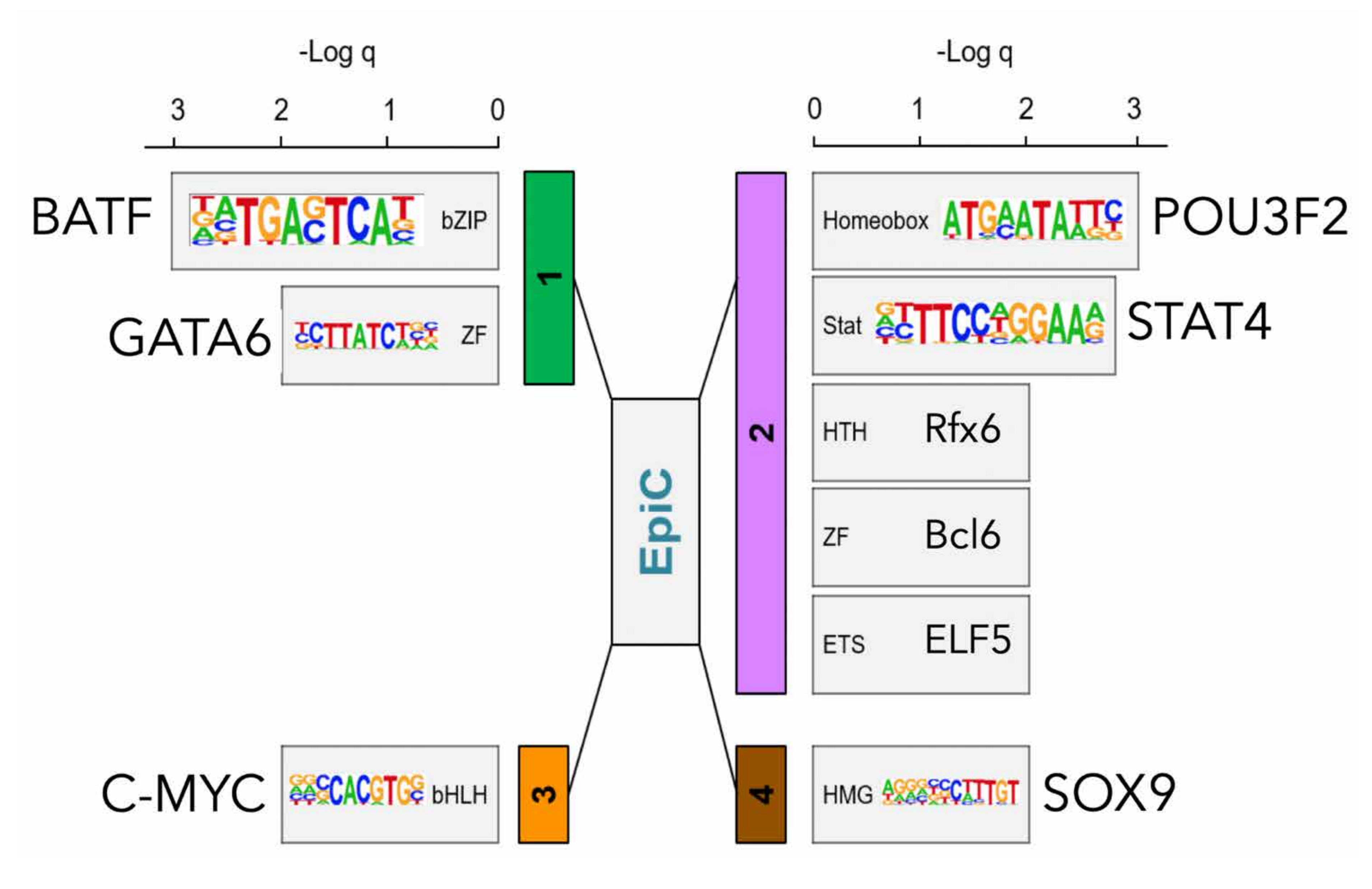




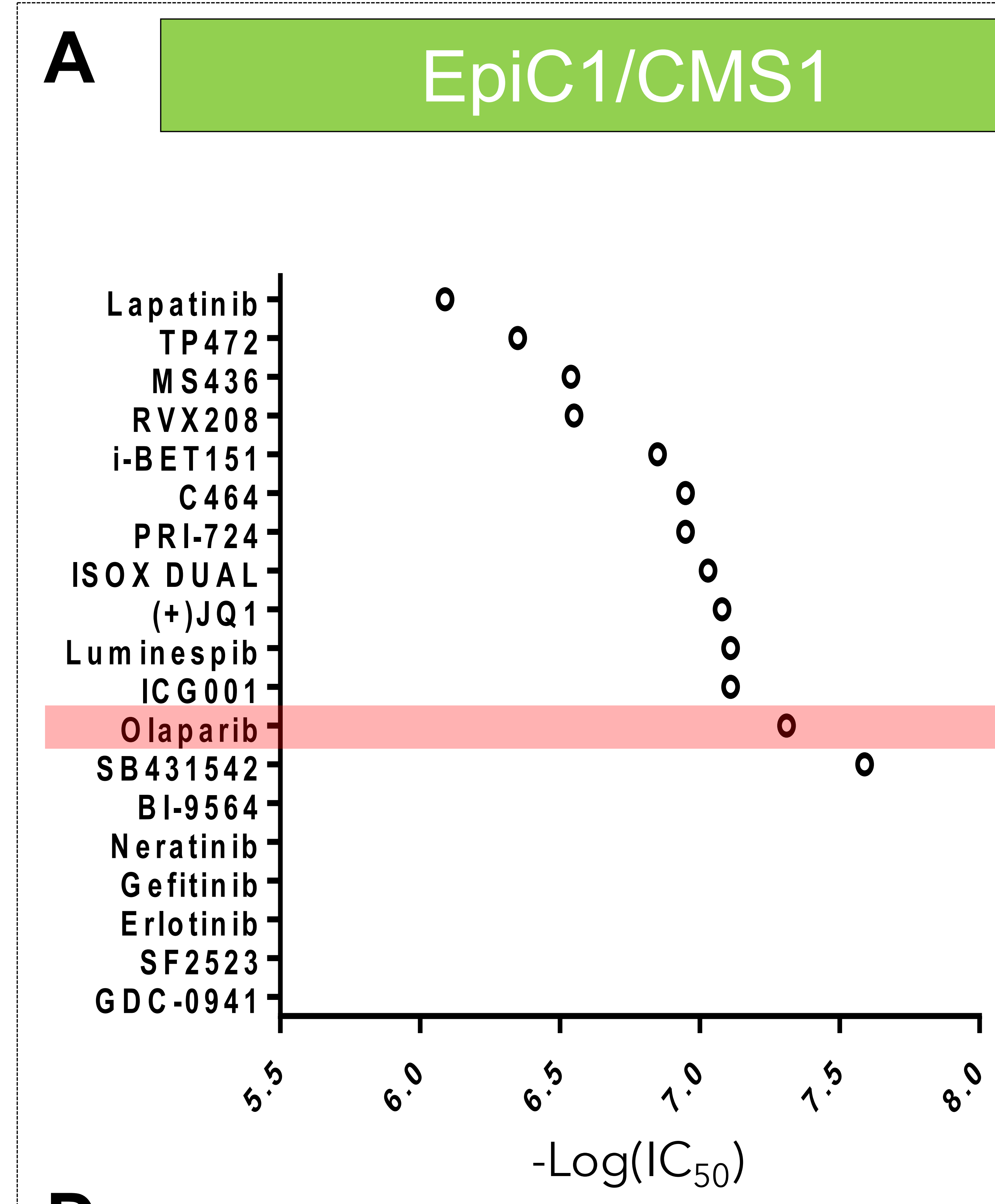

D

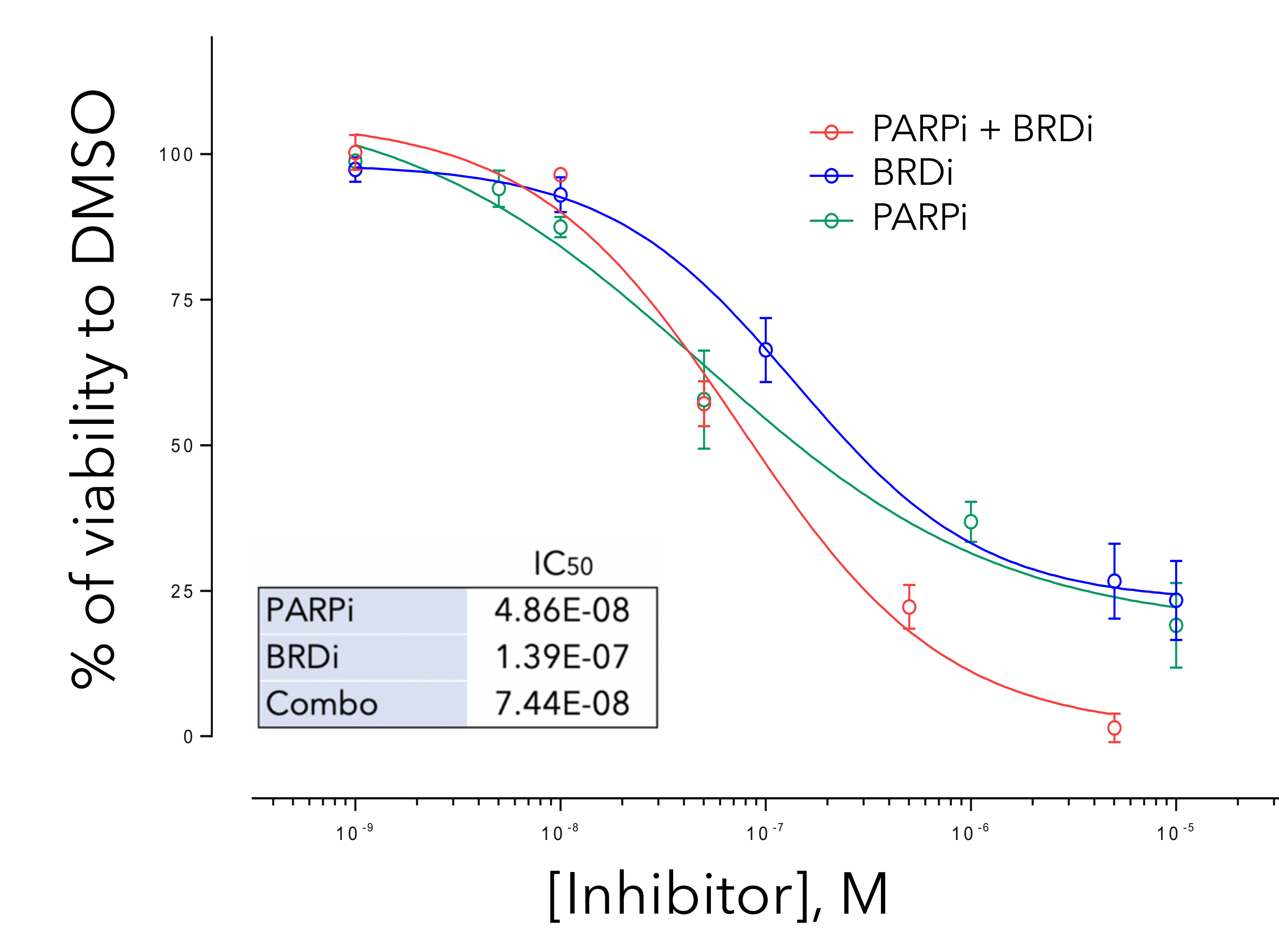

G

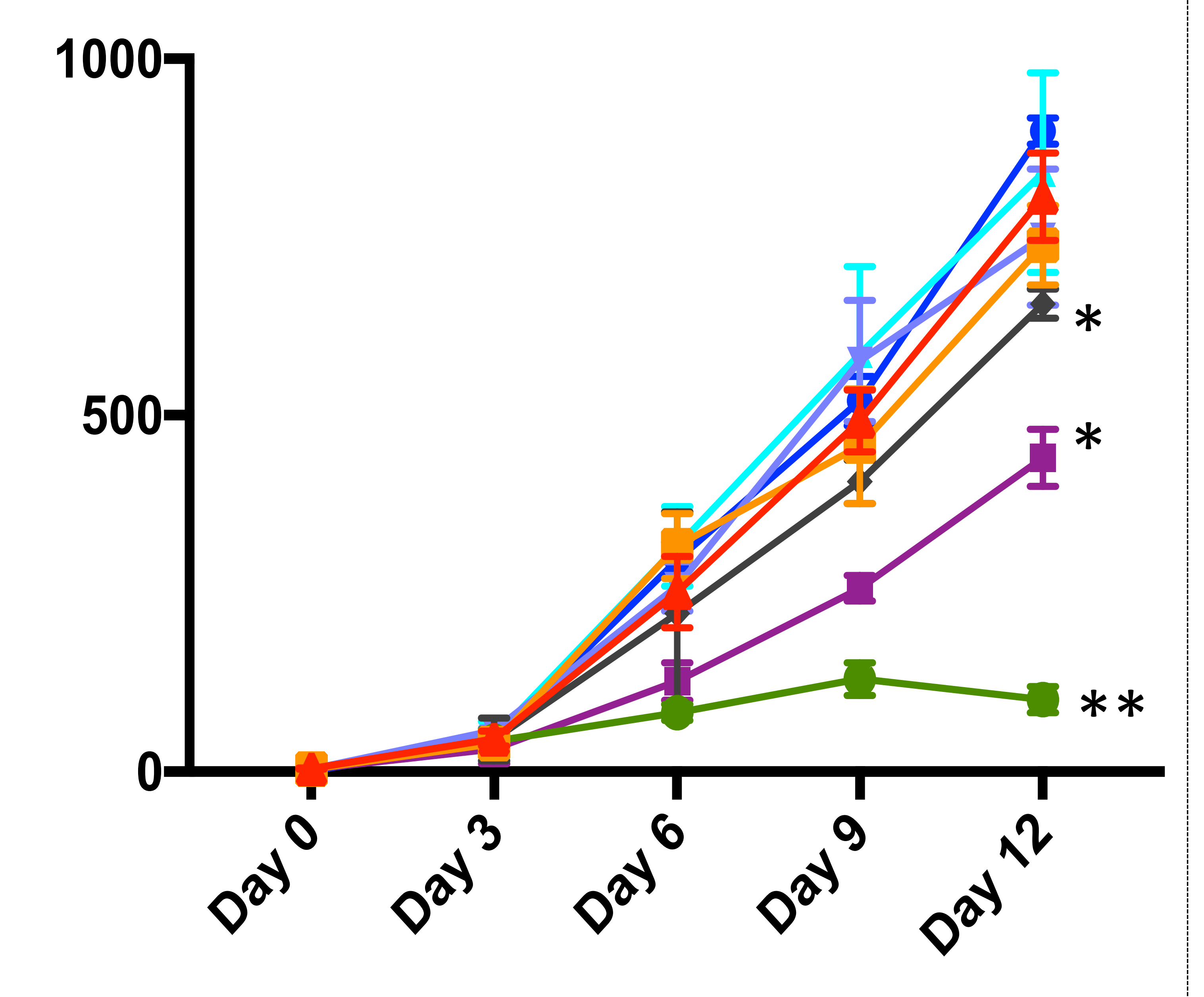

B
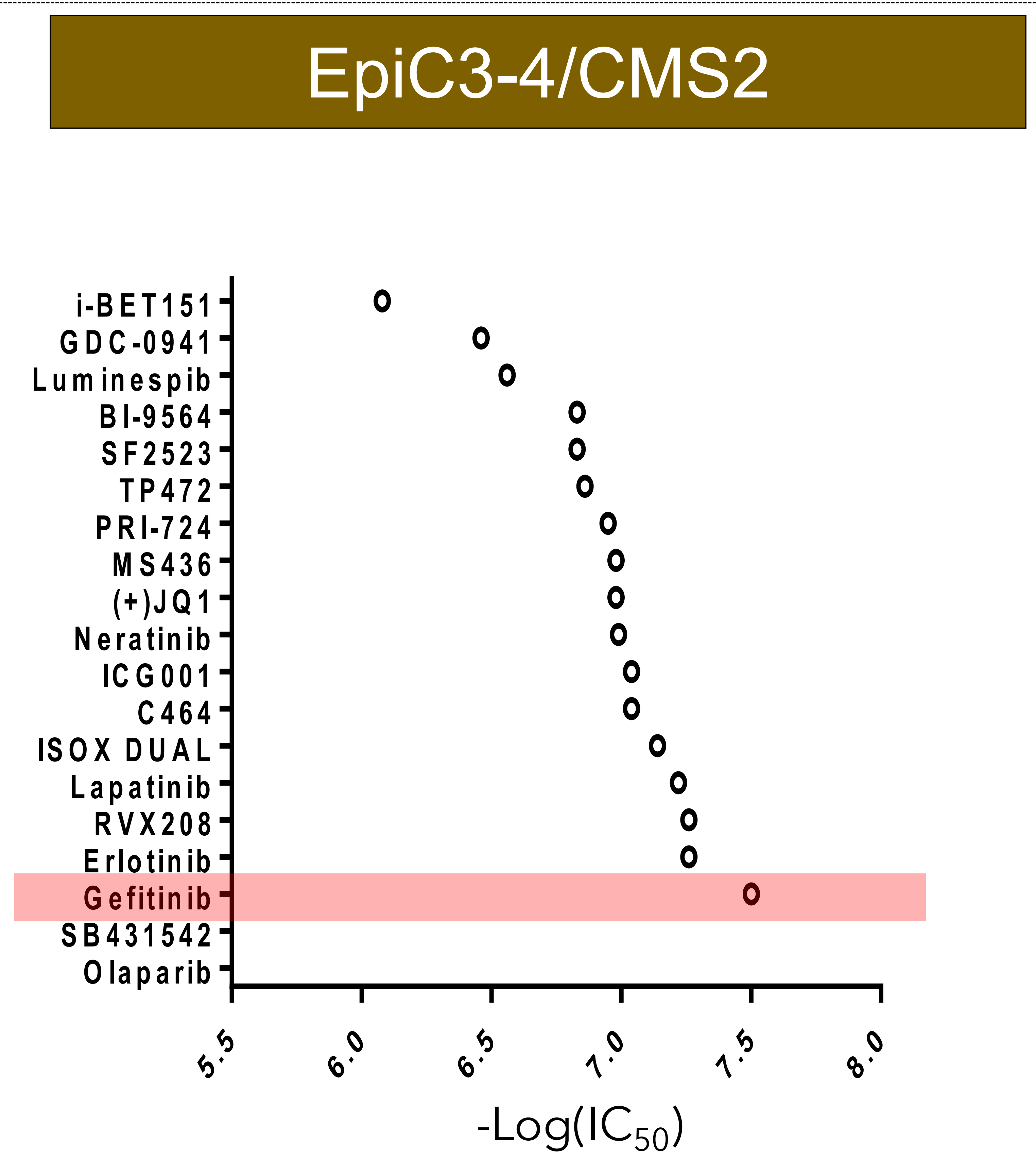

E

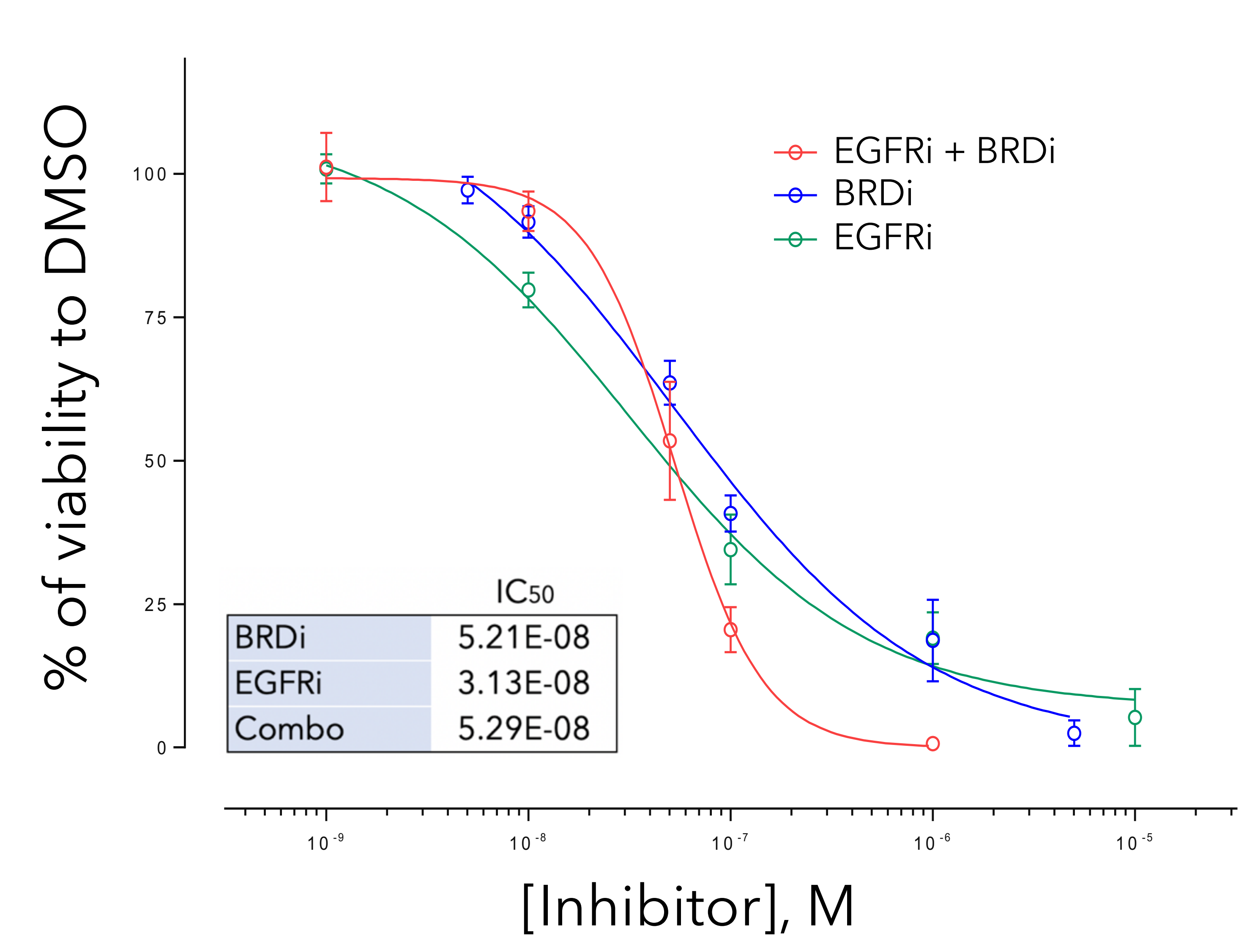

H

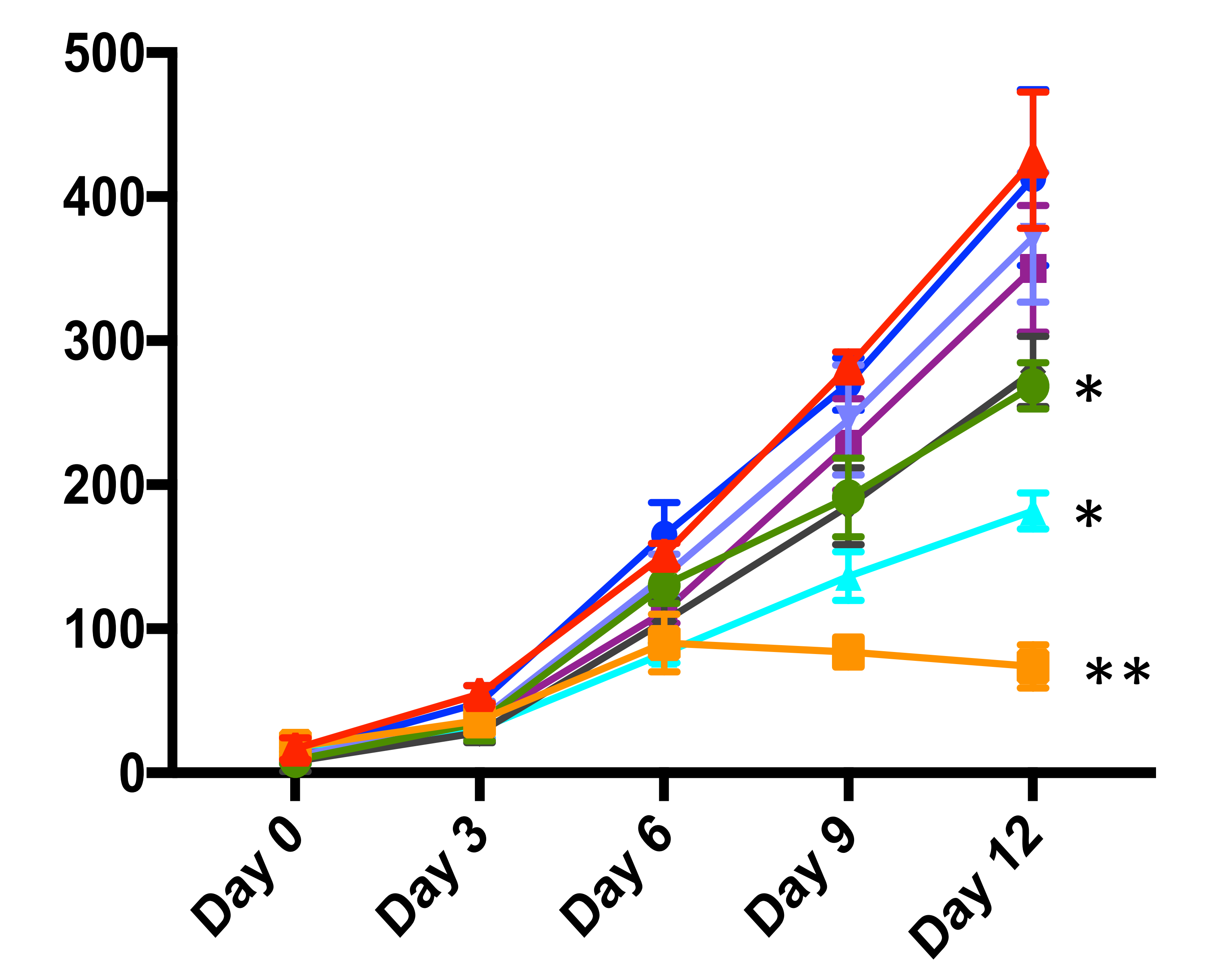

C

EpiC2/CMS4

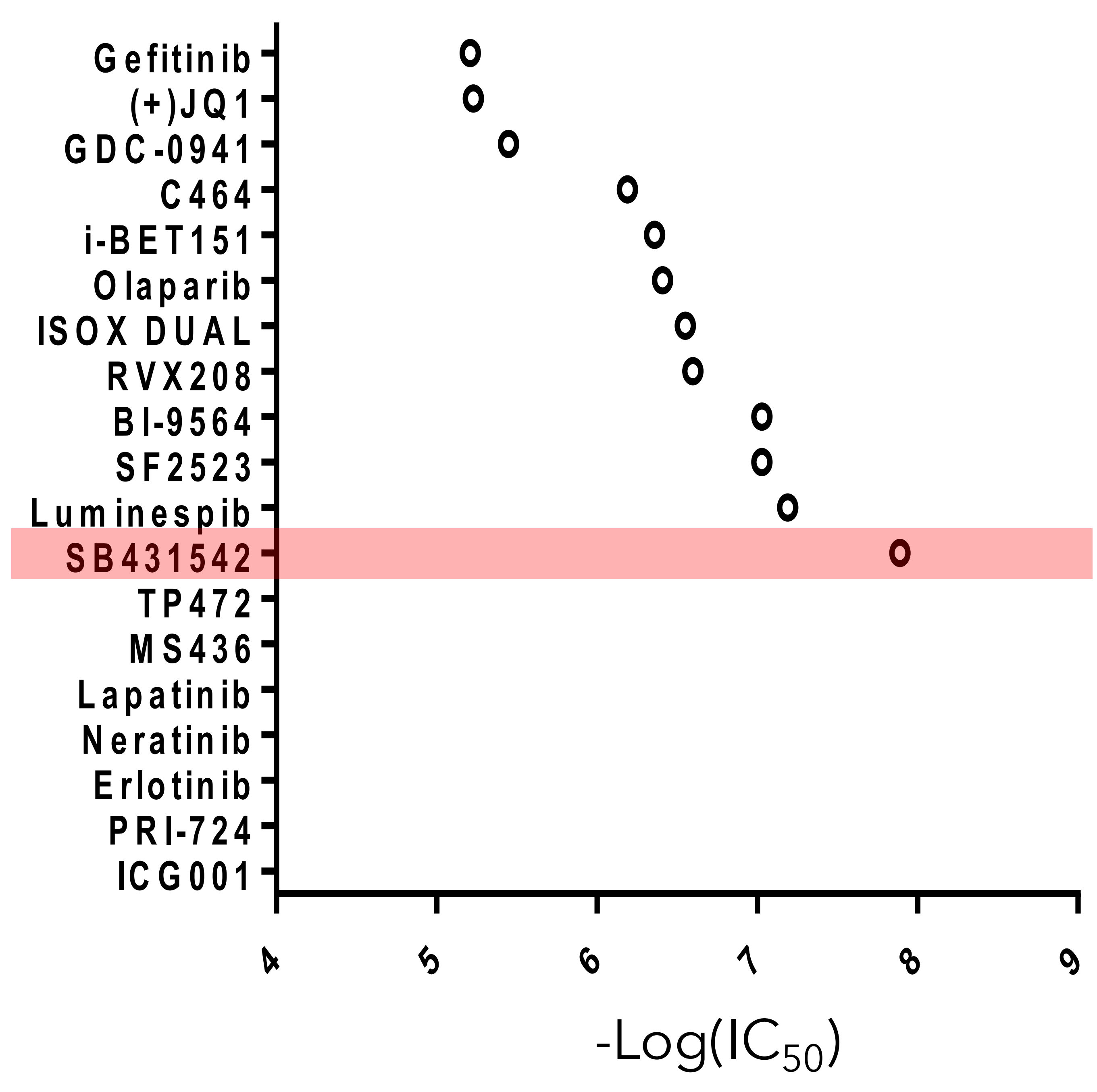

F

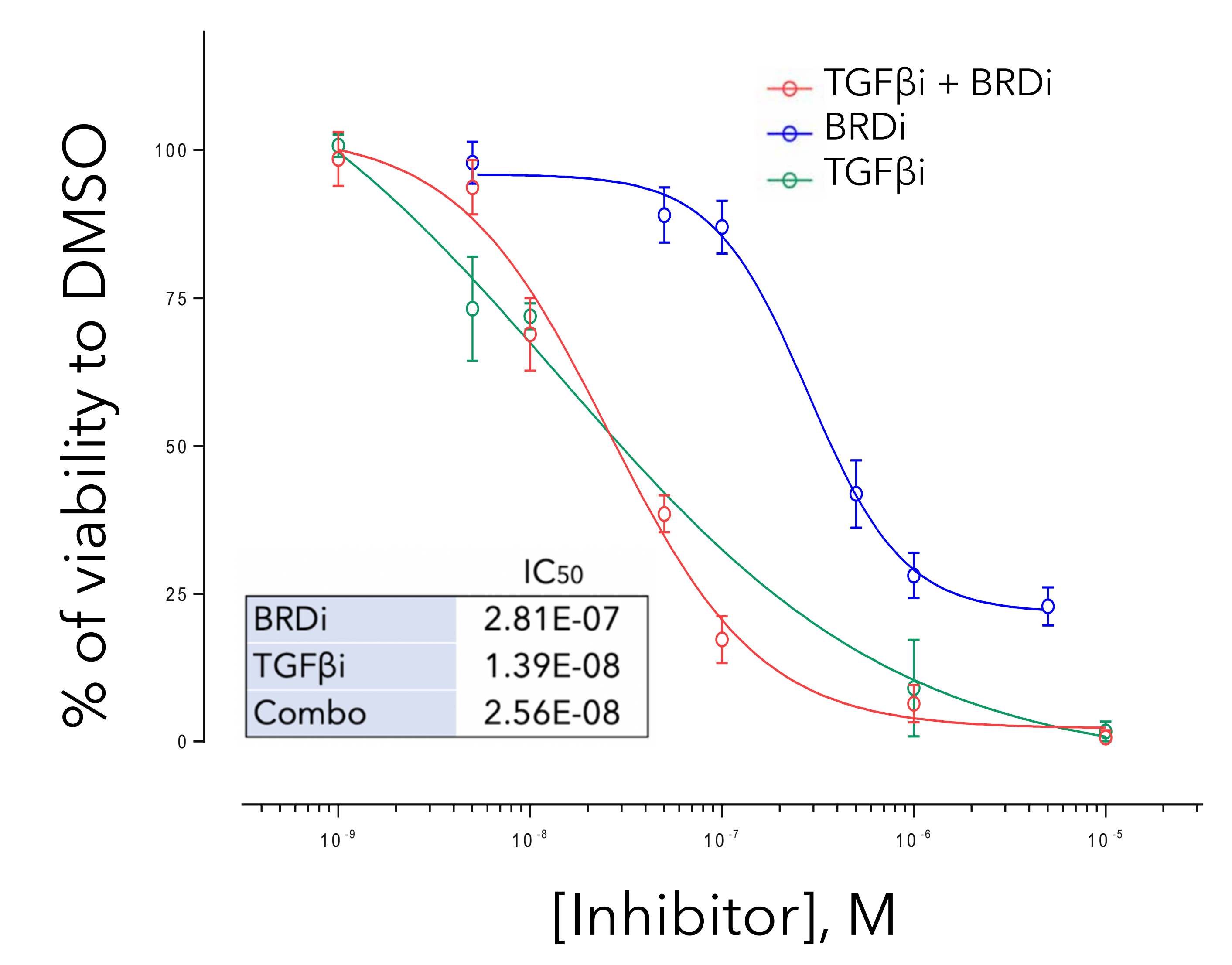

I

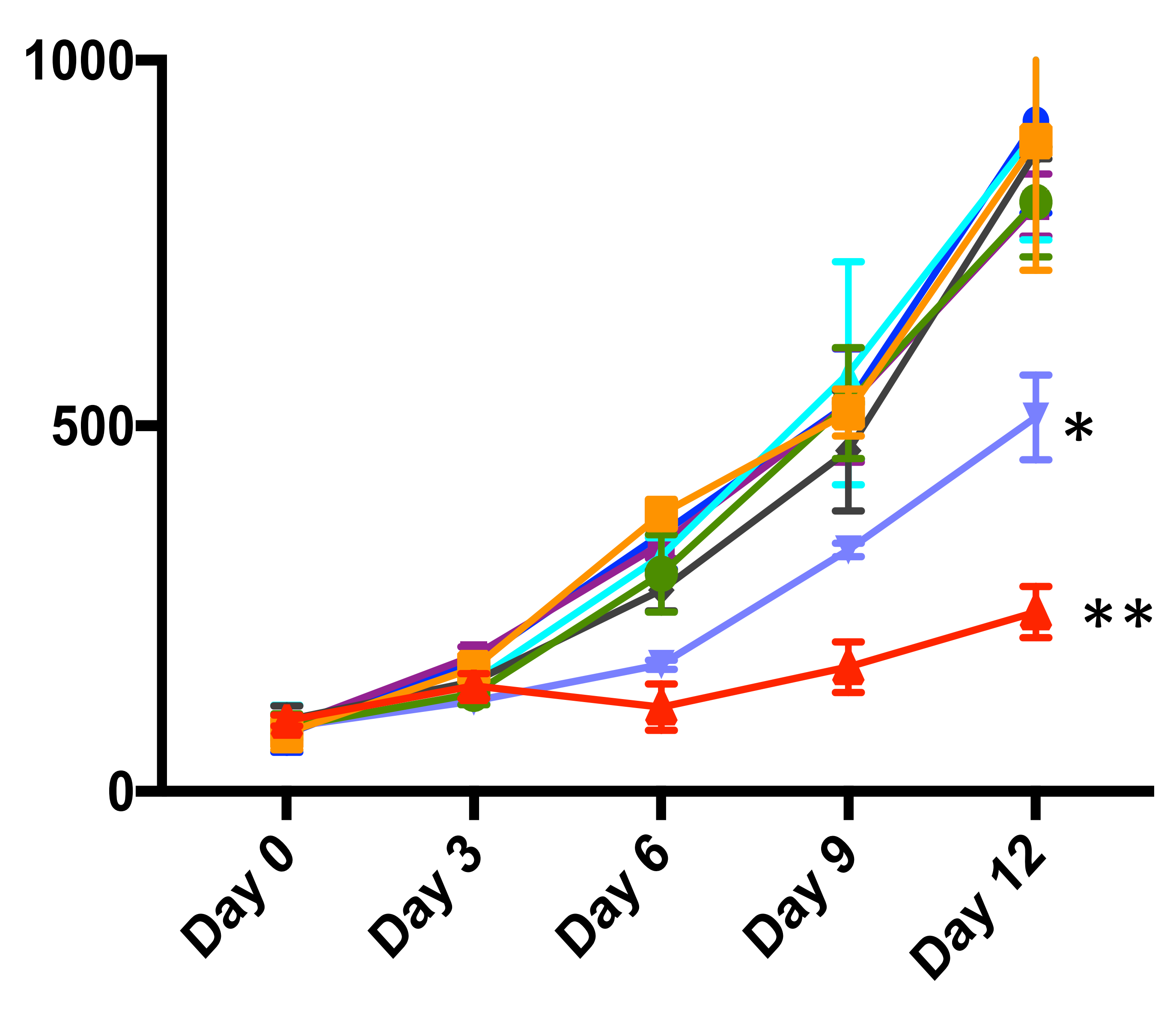


A

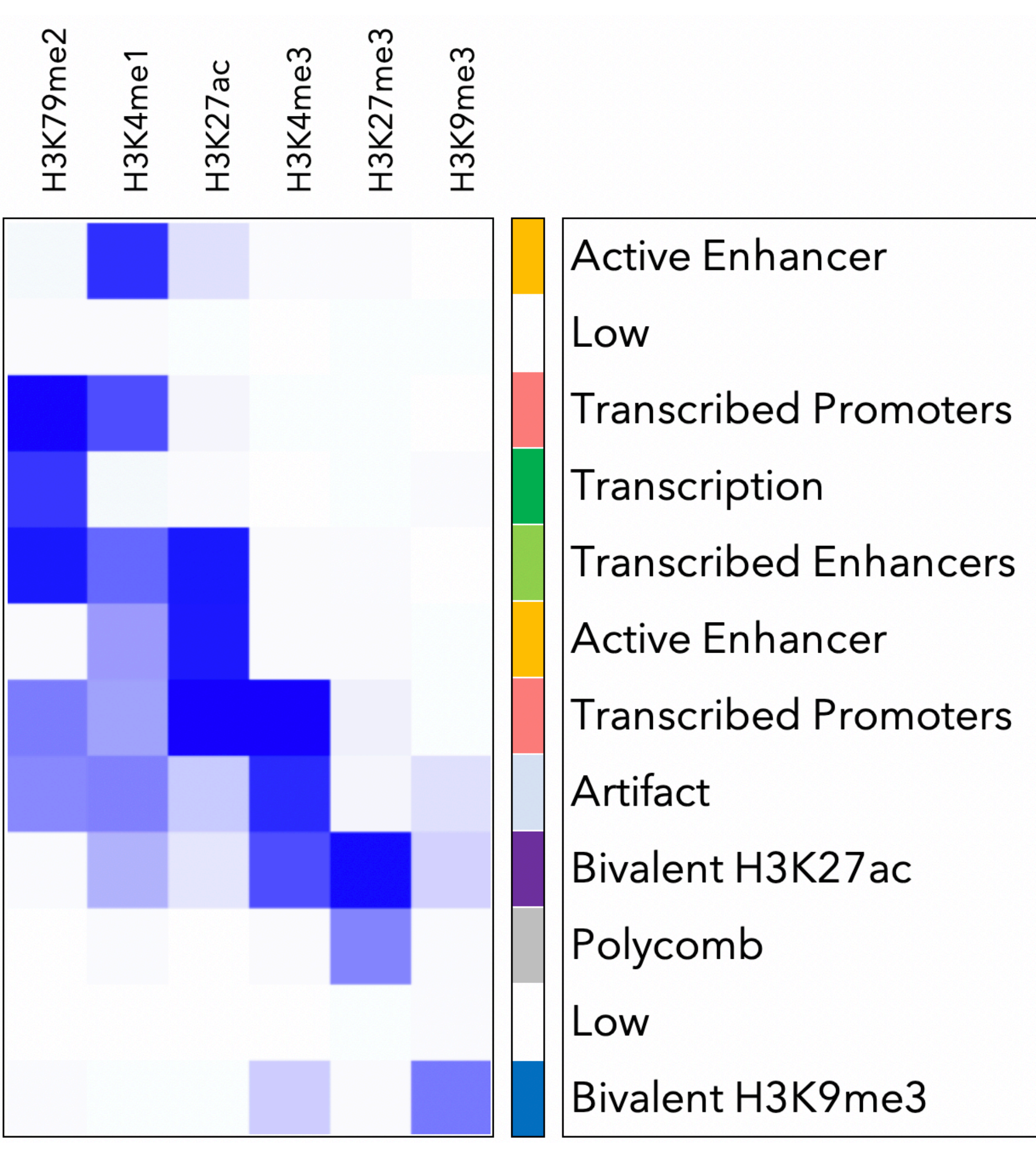

min

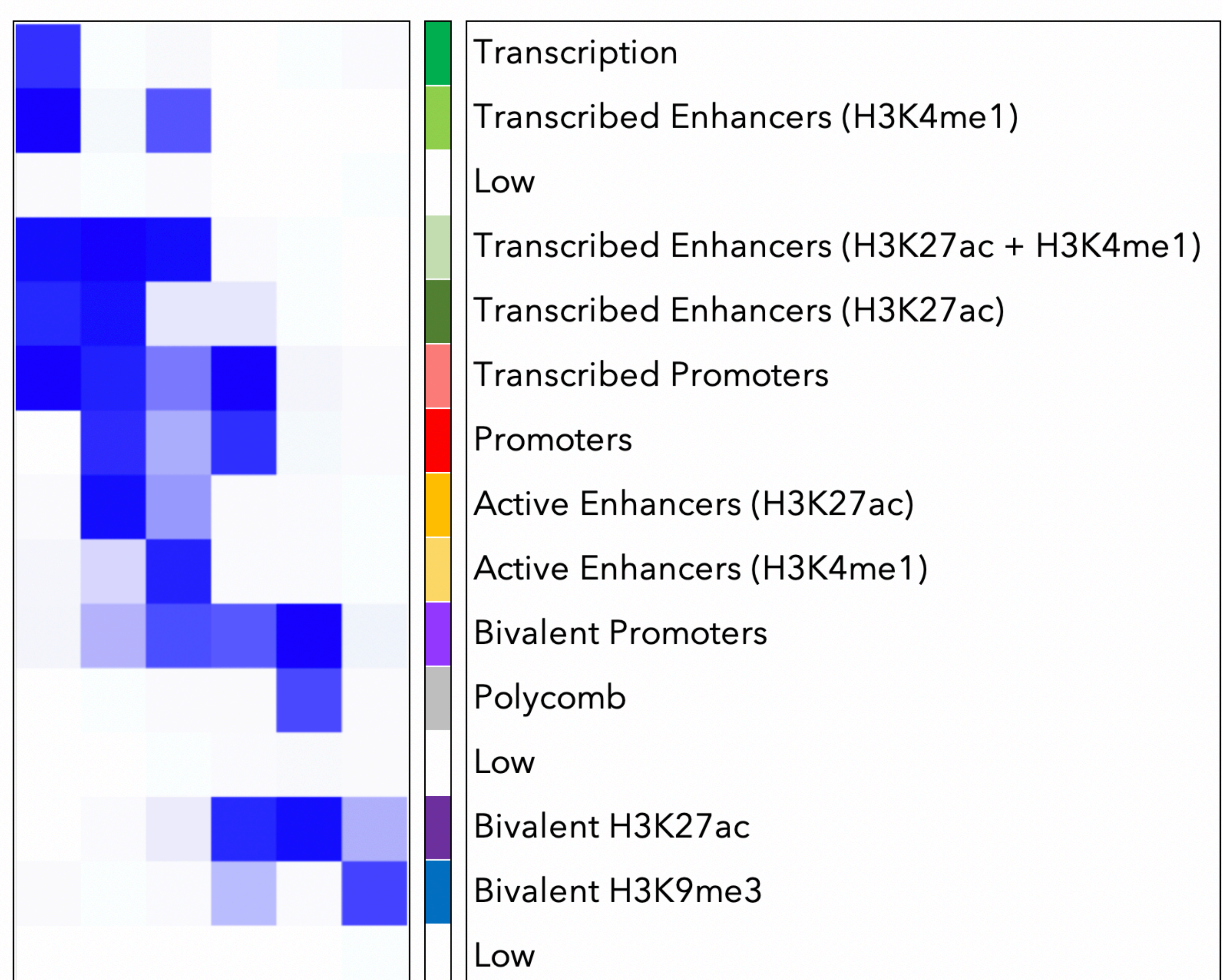

B

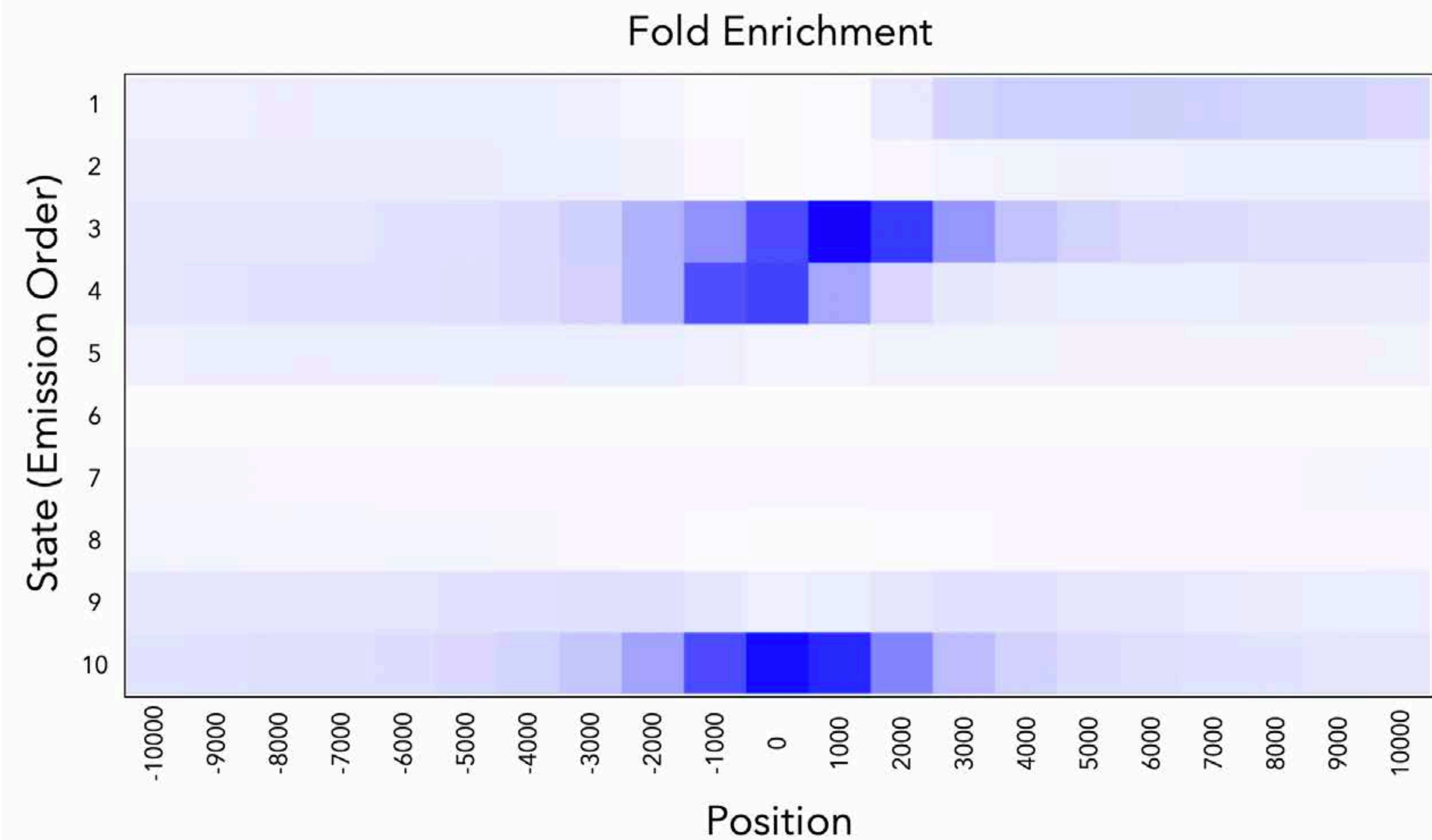

C

\section{ChromXplorer}

Sample genome

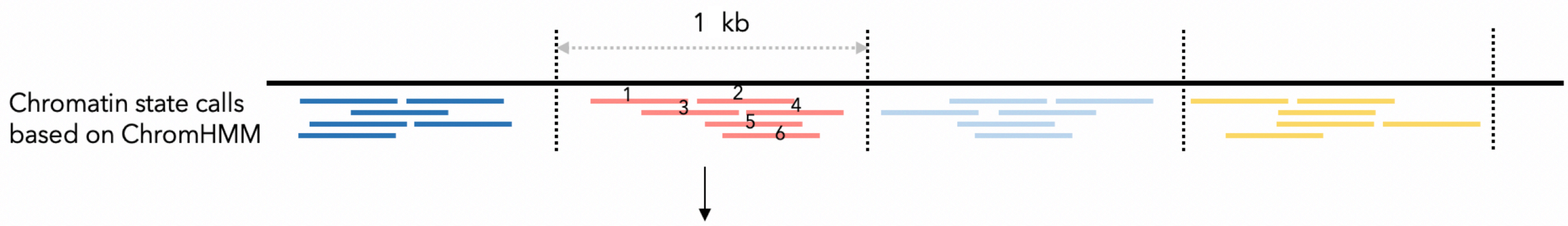

1. Segmentation of the genome to certain size bins (i.e. $1 \mathrm{~kb}$ window)

2. Identification of the frequency of a certain chromatin state being called in that window

3. Preparation of frequency matrix for all samples, Mann-Whitney U test $(p<0.05)$

D

State 1
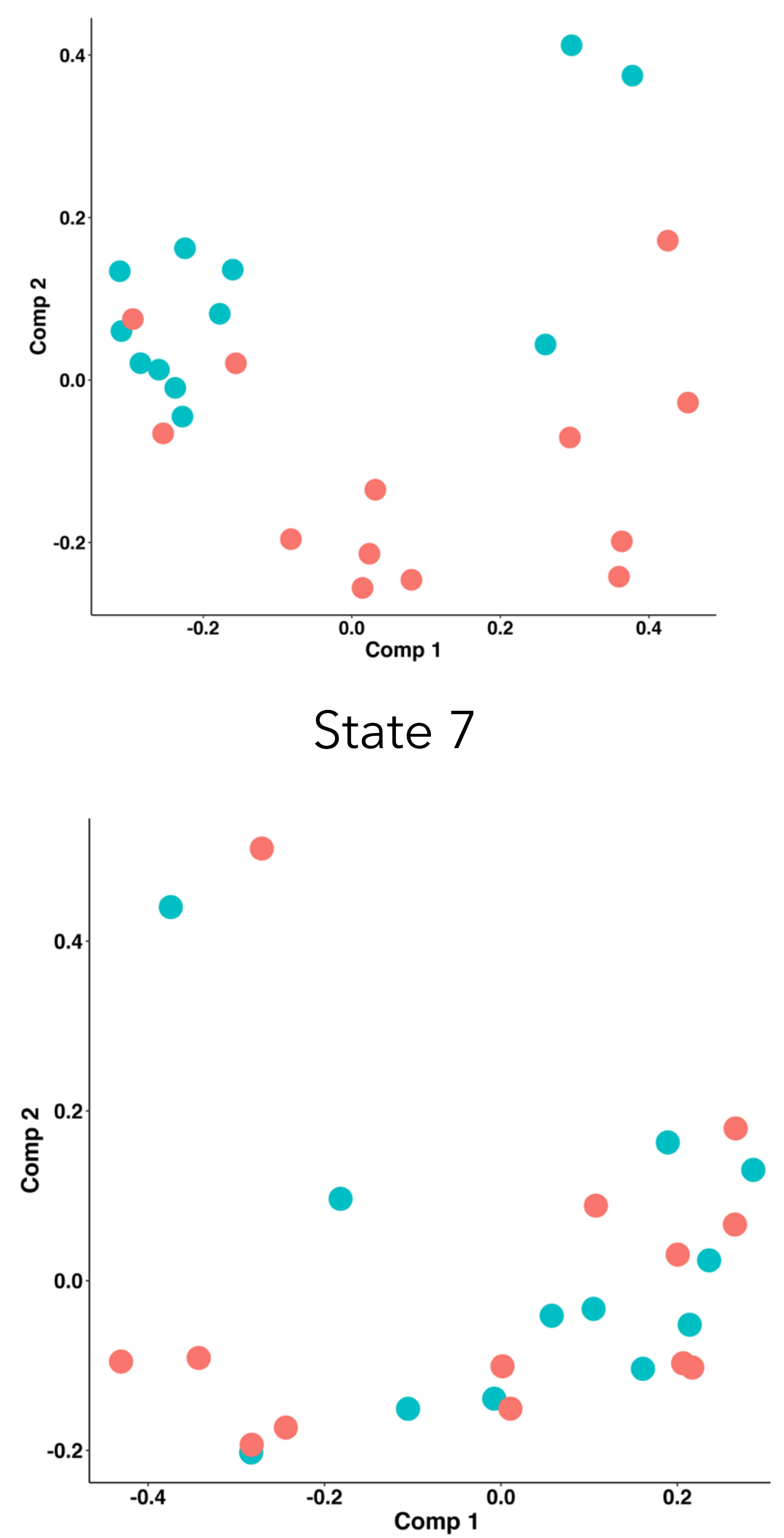

State 2
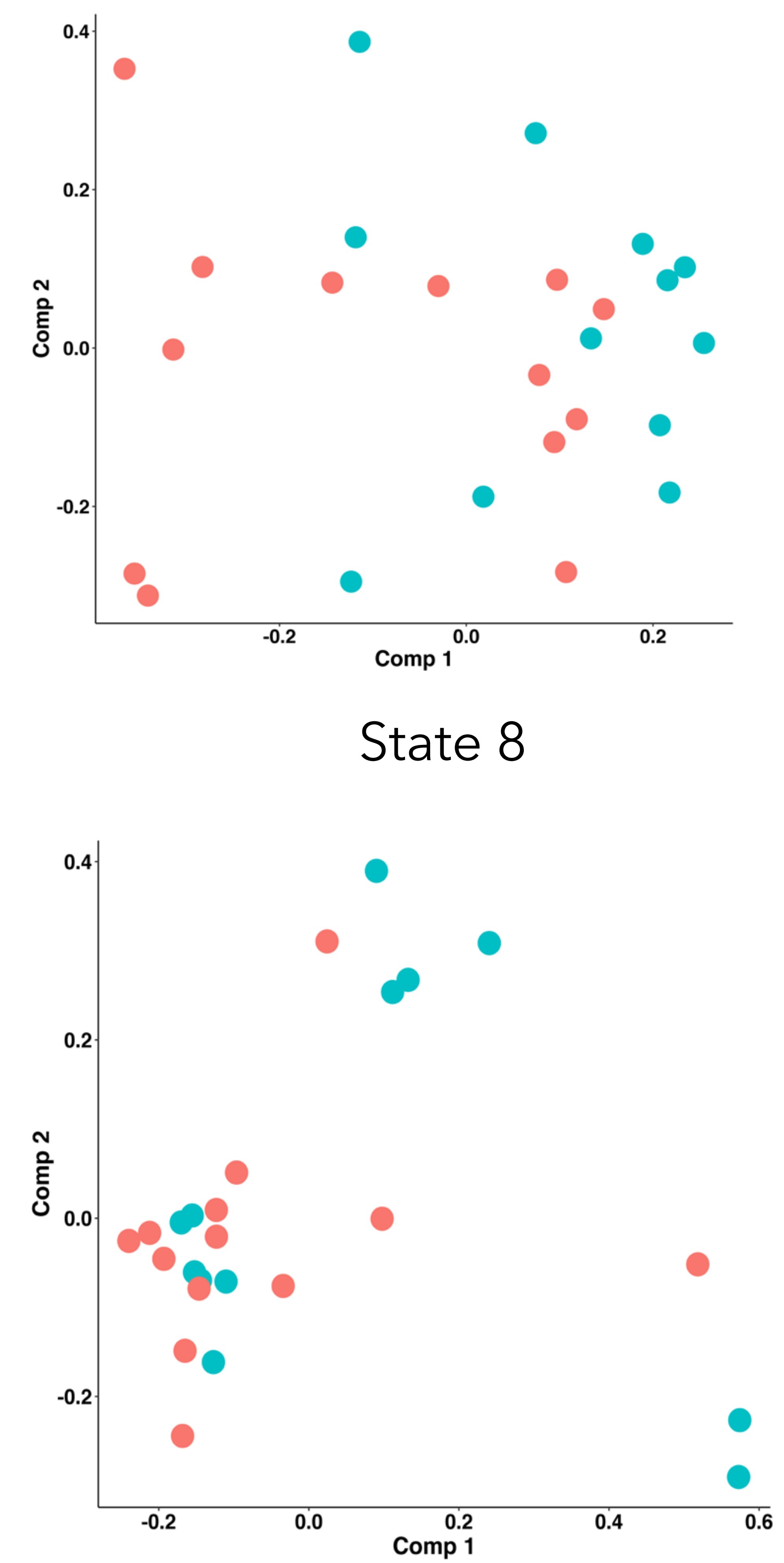

State 4
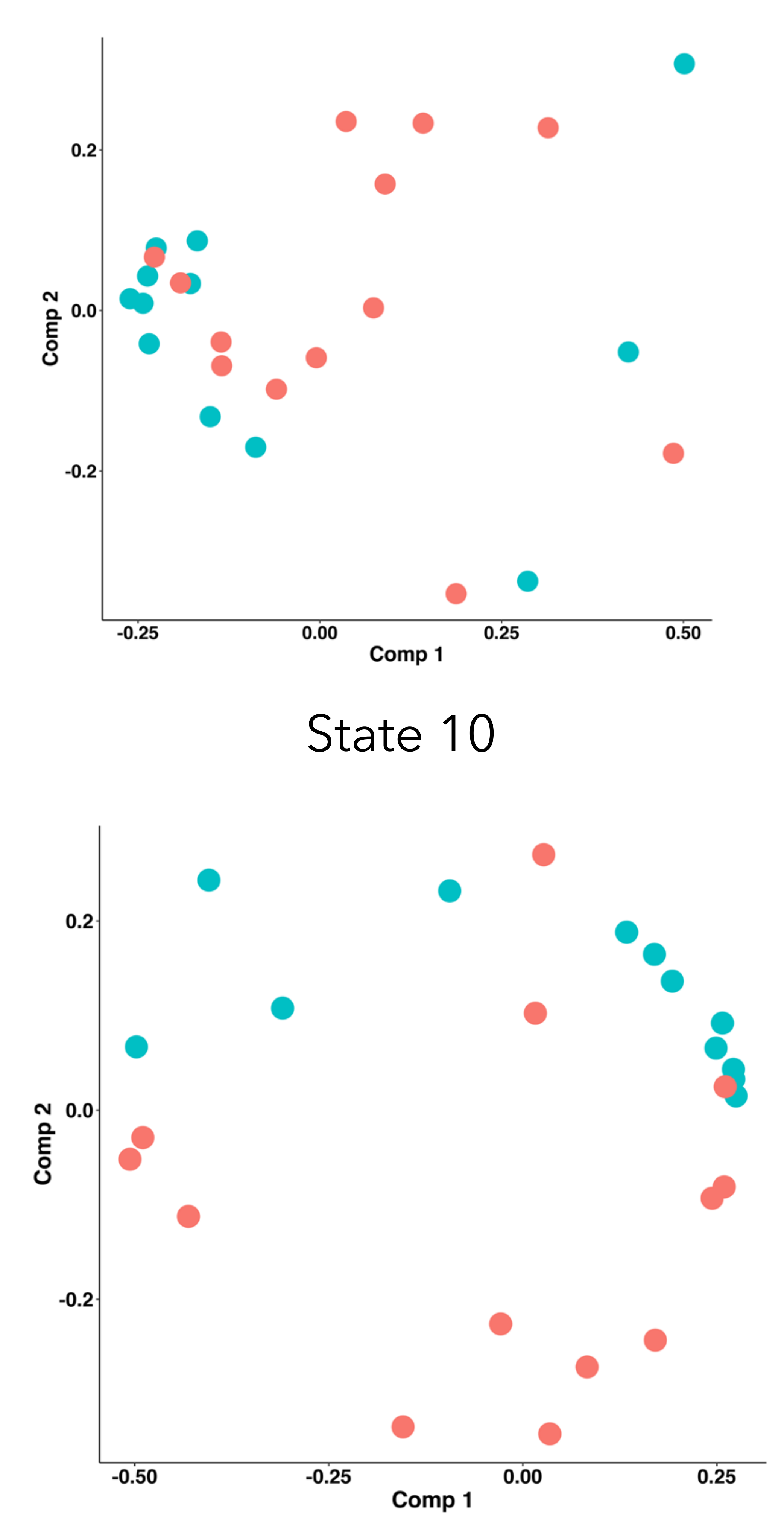

State 6

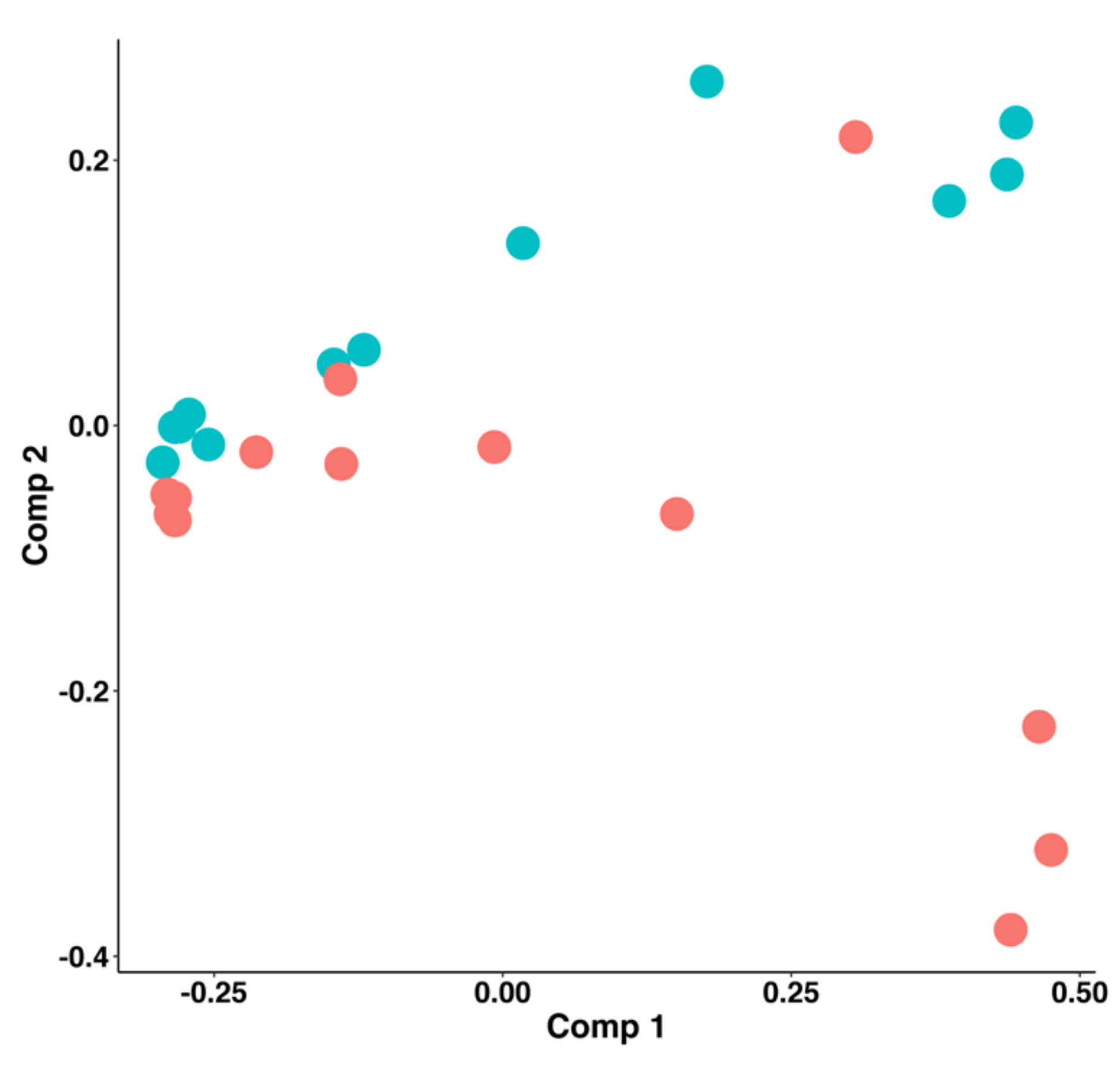

E
State 6

(12-state Model)
State 8 (15-state Model)
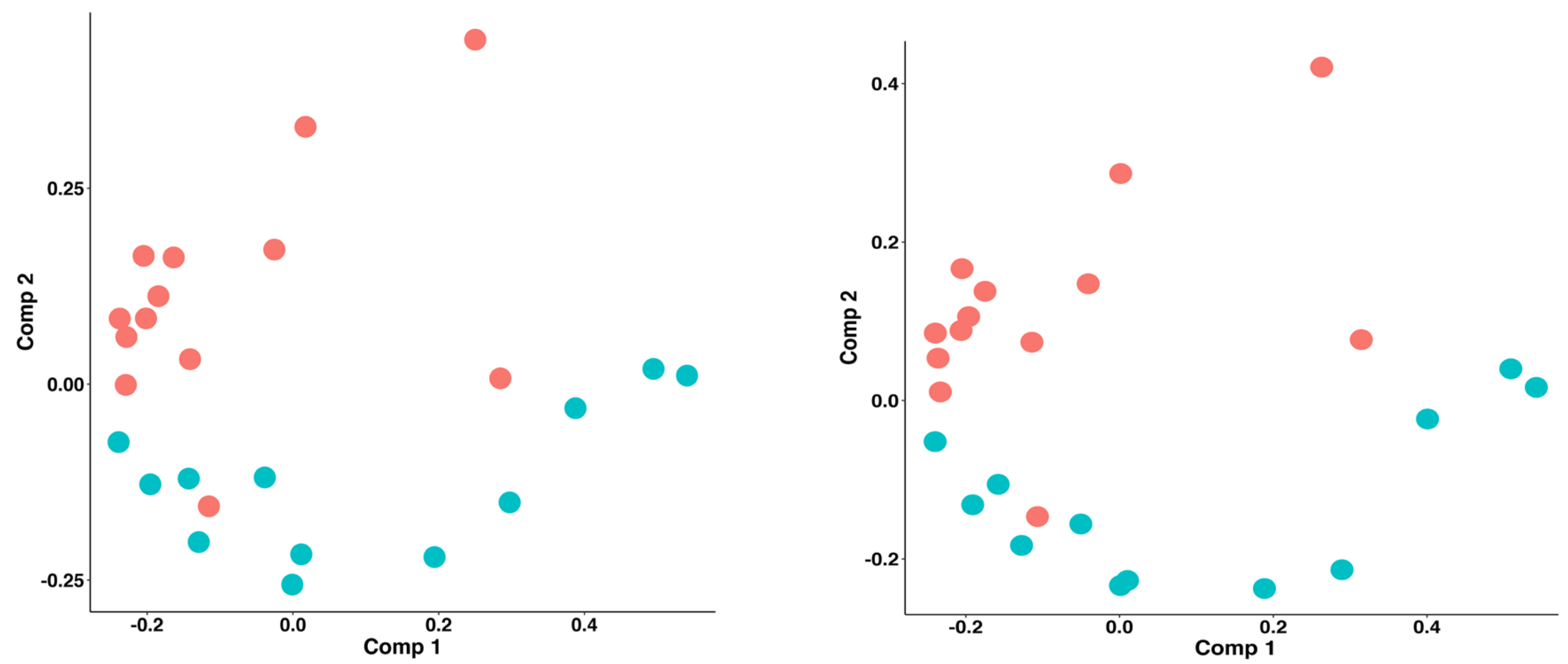

$\mathbf{F}$

State 5: Active Enhancer

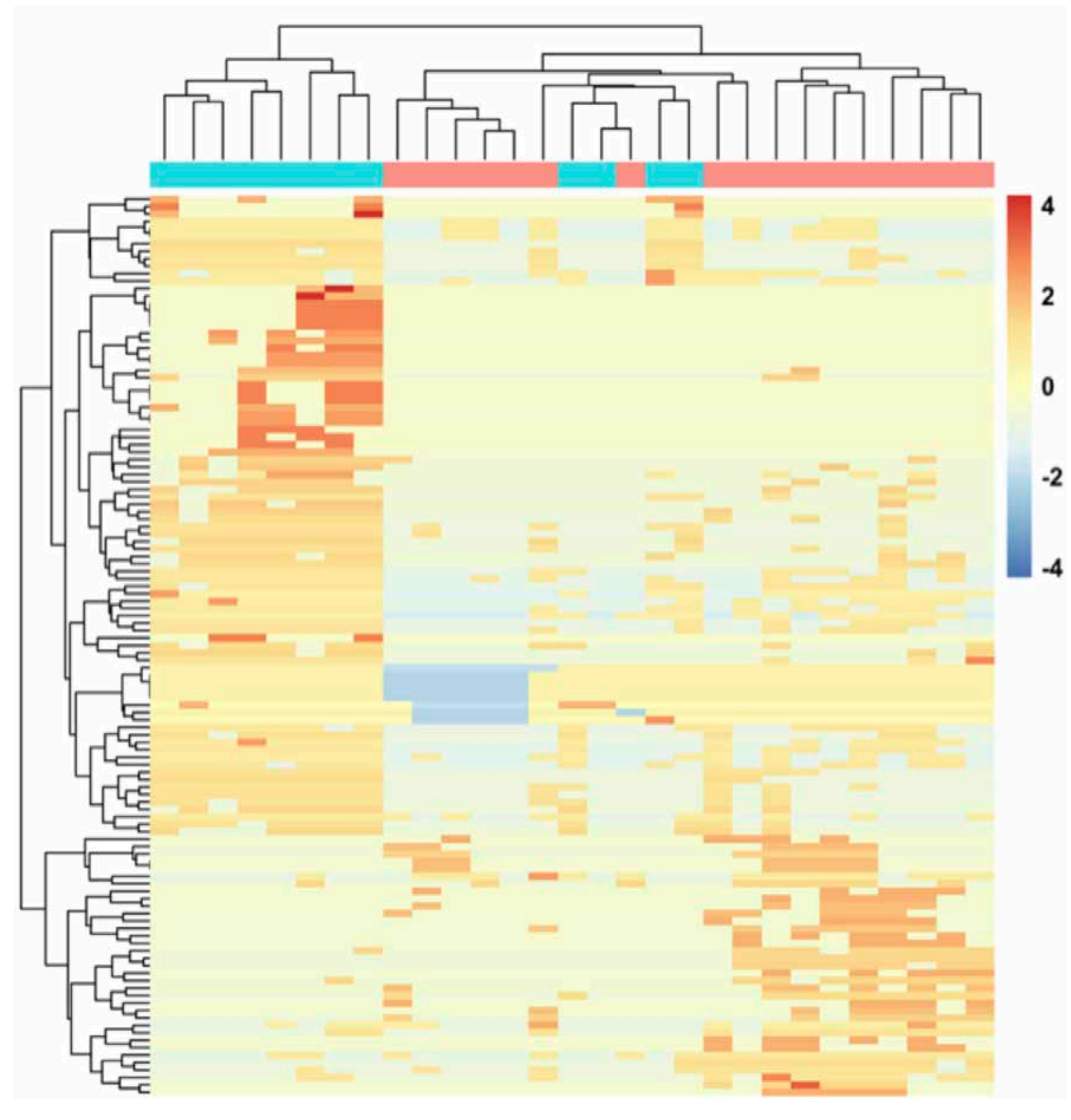

Tumor

Normal
Tumor

Normal 
Figure S2

A

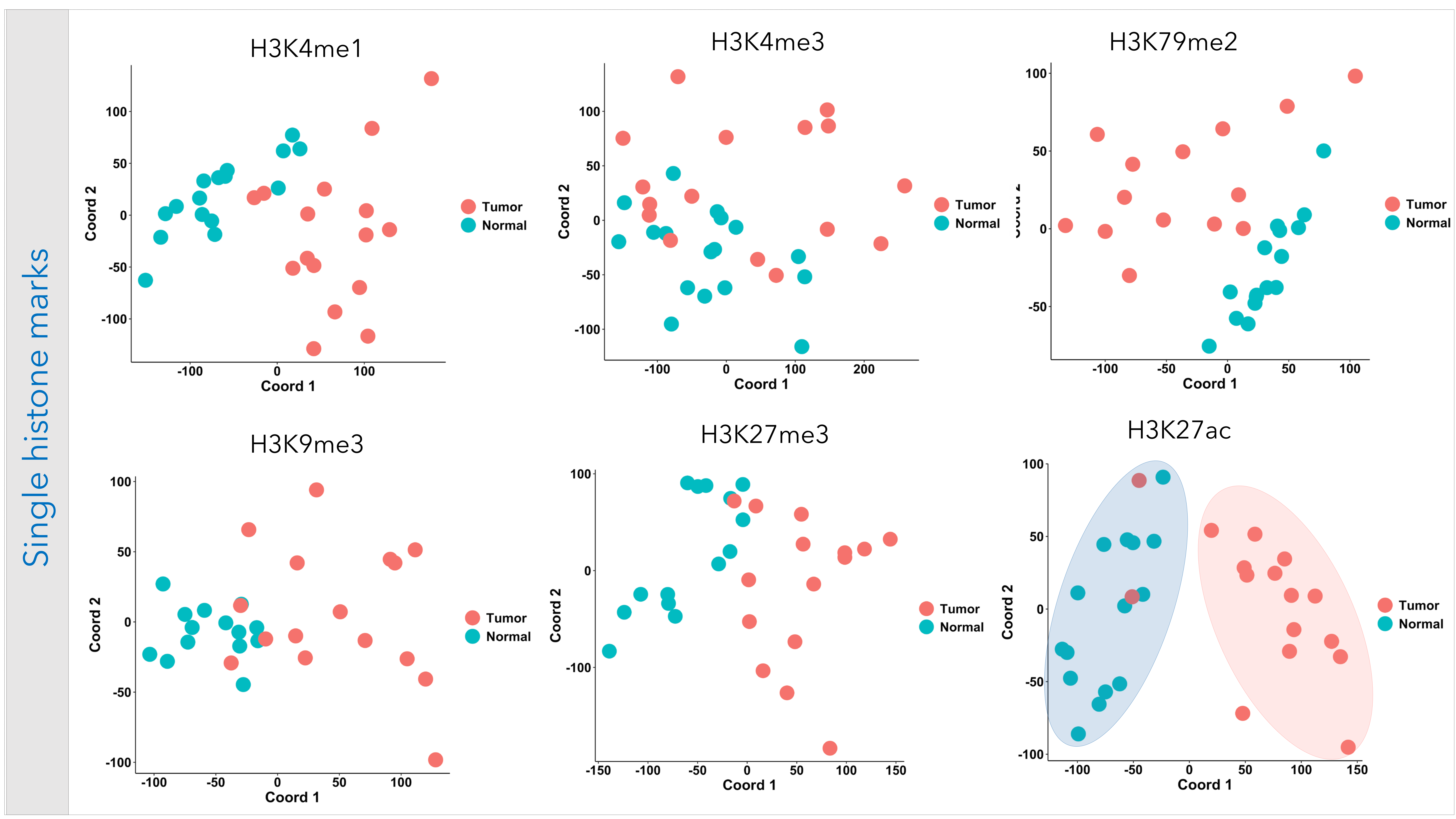

B

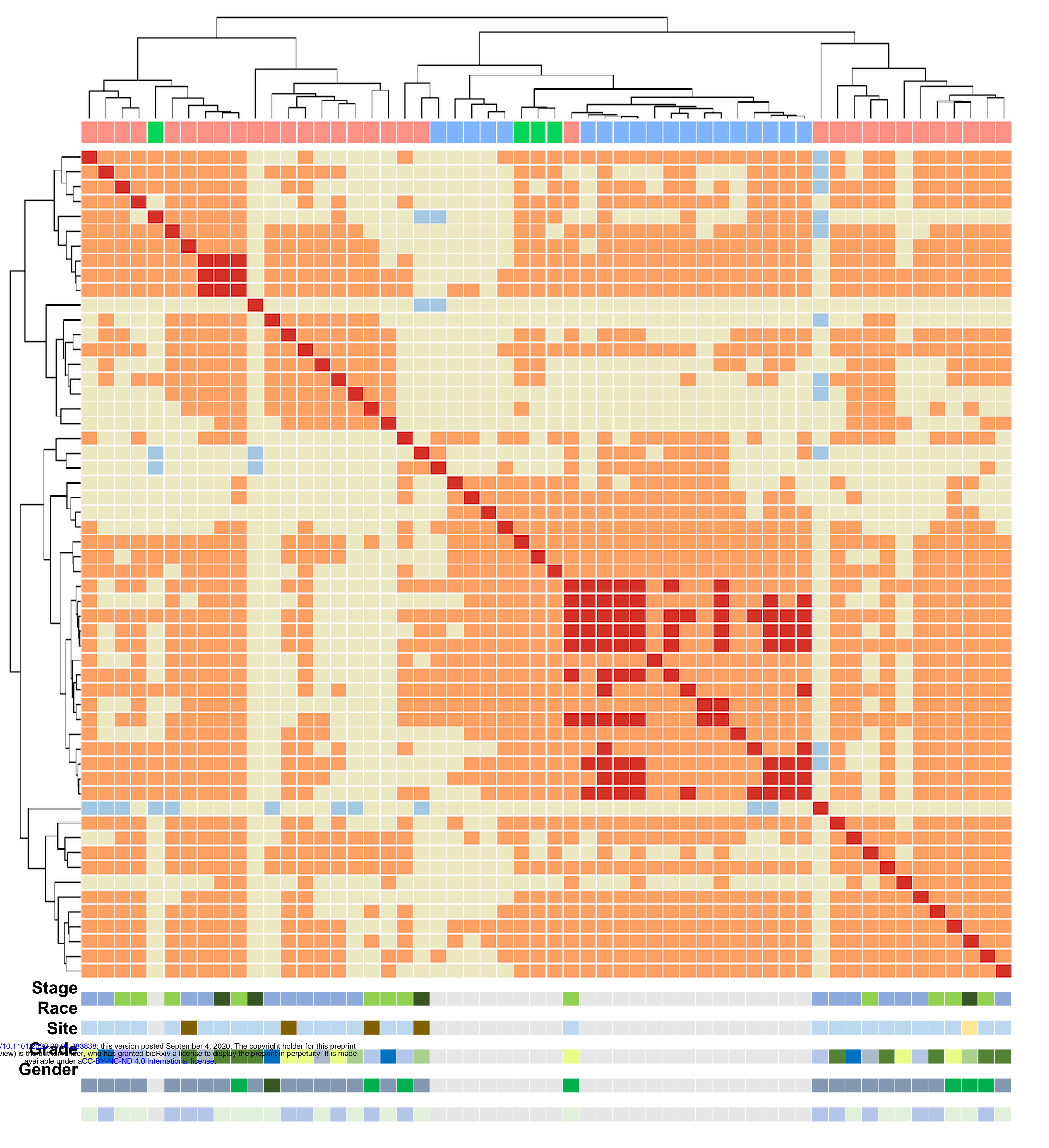

C

H3K27ac occupancy plot in normal/ adenoma/ CRC

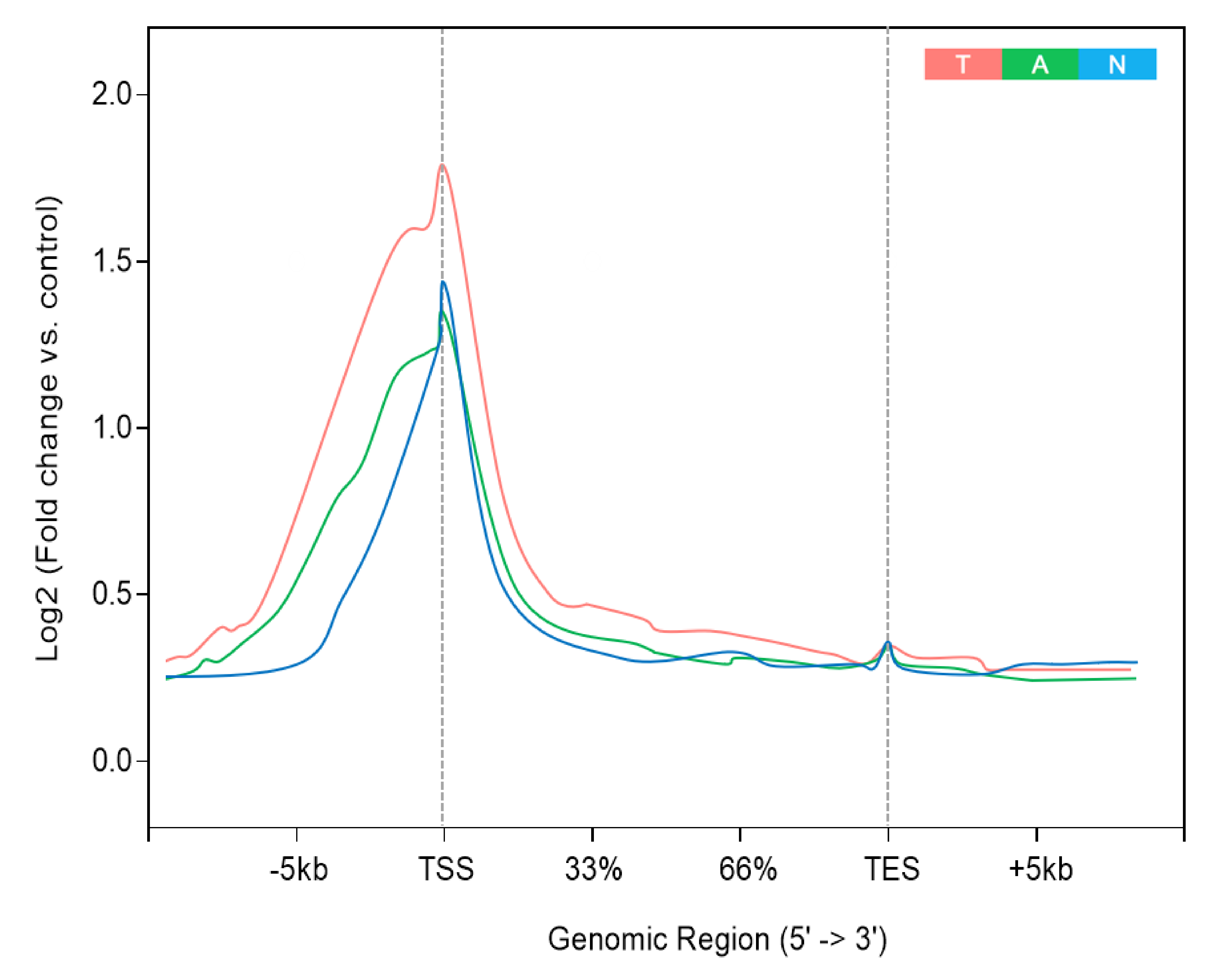

Normal

Adenoma

Tumor

\begin{tabular}{|c|c|c|}
\hline Stage 2 & Well diffr & Ascending colon \\
\hline Stage 3 & Mod diffr & Descending colon \\
\hline Stage 4 & Poor diffr & Sigmoid colon \\
\hline \multirow[t]{2}{*}{ NA } & NA & Transverse colon \\
\hline & & R-S junction \\
\hline Male & Hispanic & Anus \\
\hline \multirow[t]{2}{*}{ Female } & White & Caecum \\
\hline & Black & Rectum \\
\hline
\end{tabular}


Figure S3

A

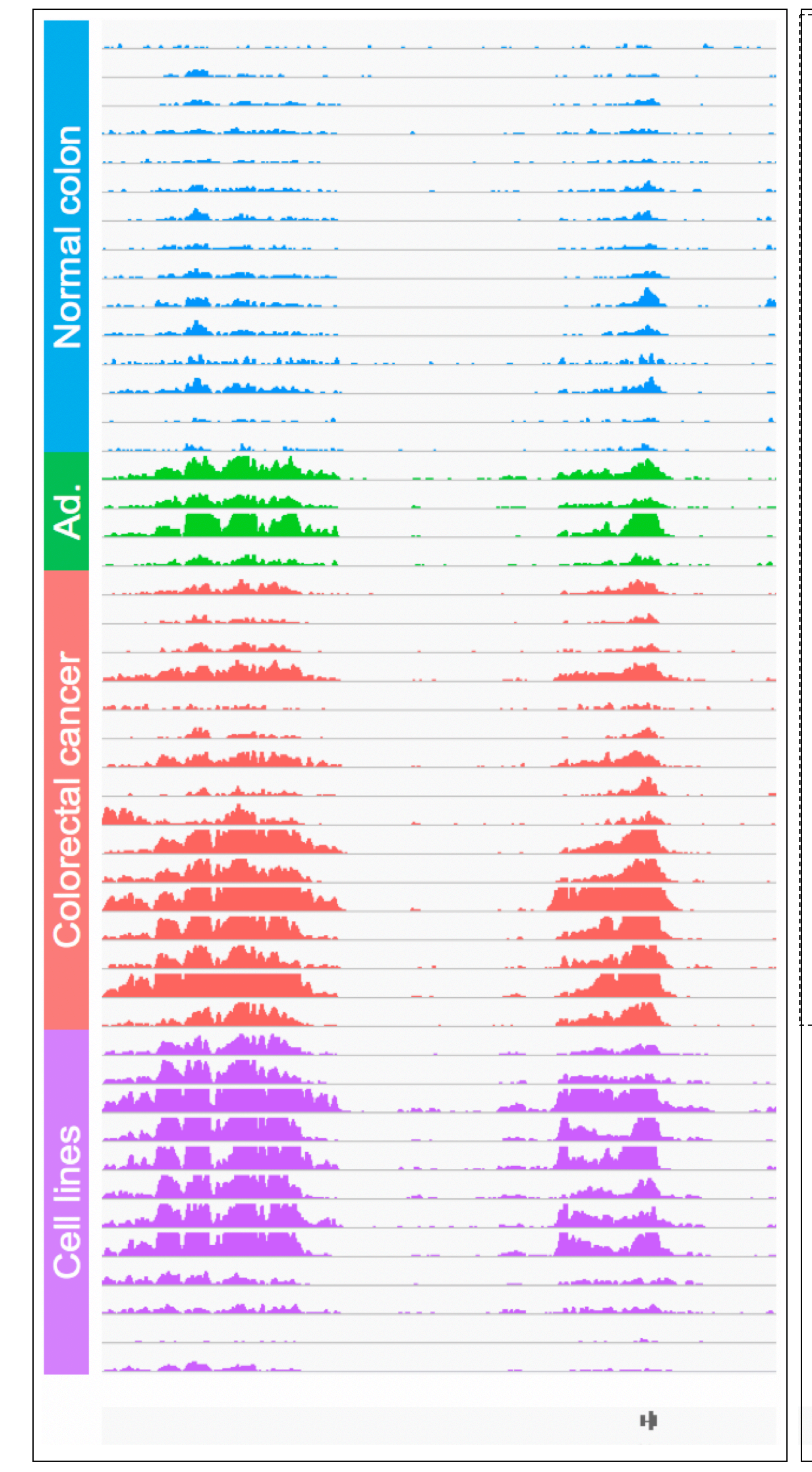

ASCL2

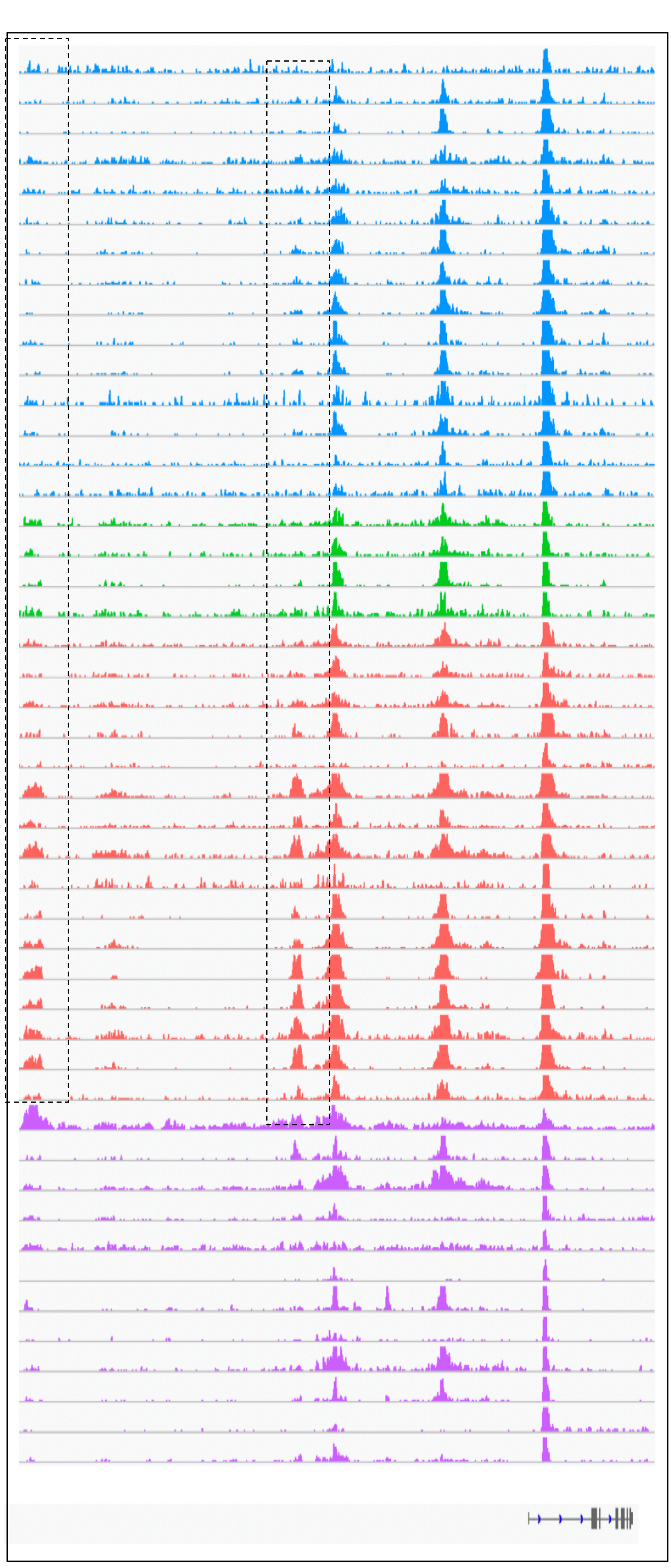

$\beta$-CATENIN

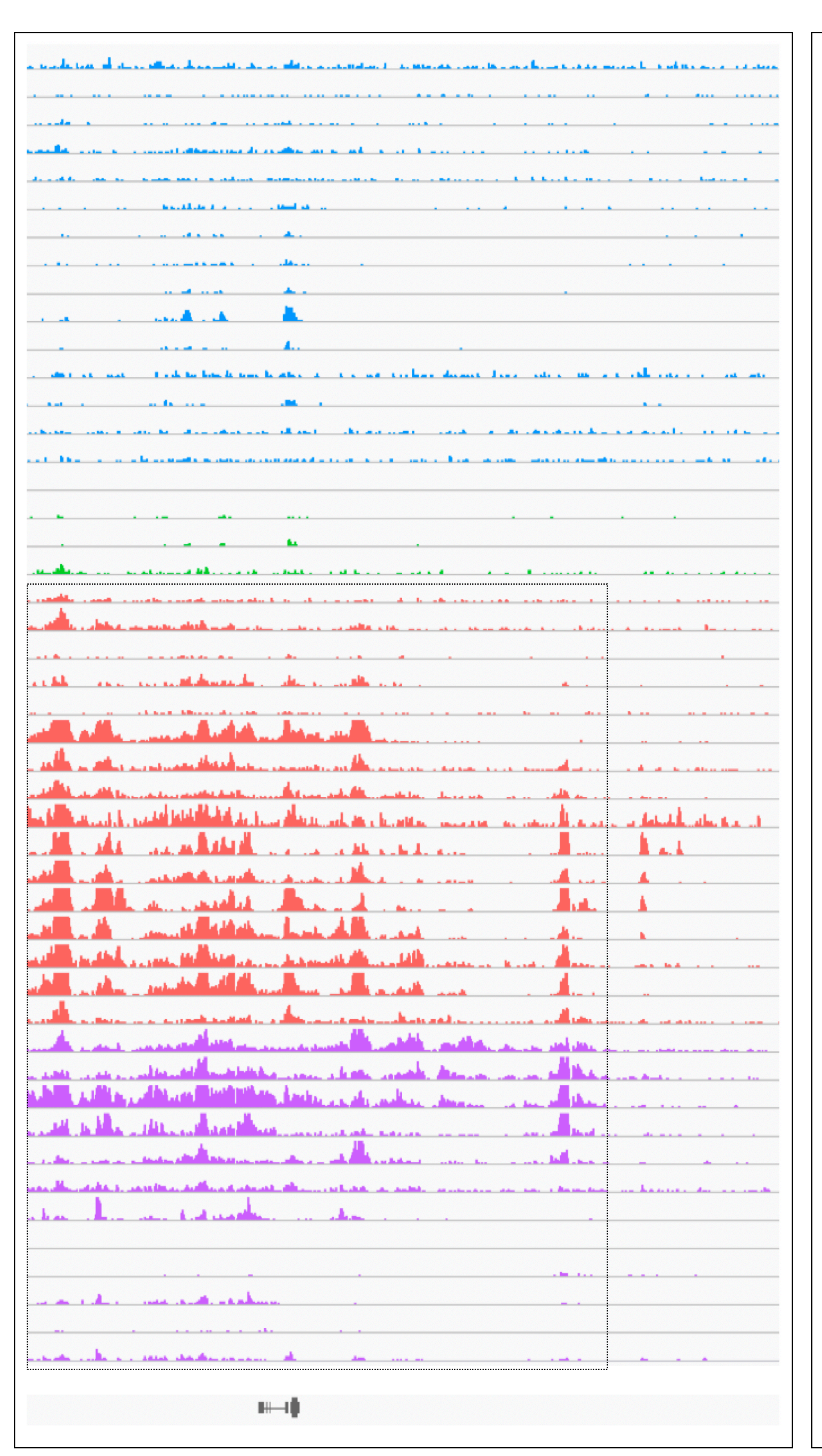

FZD10

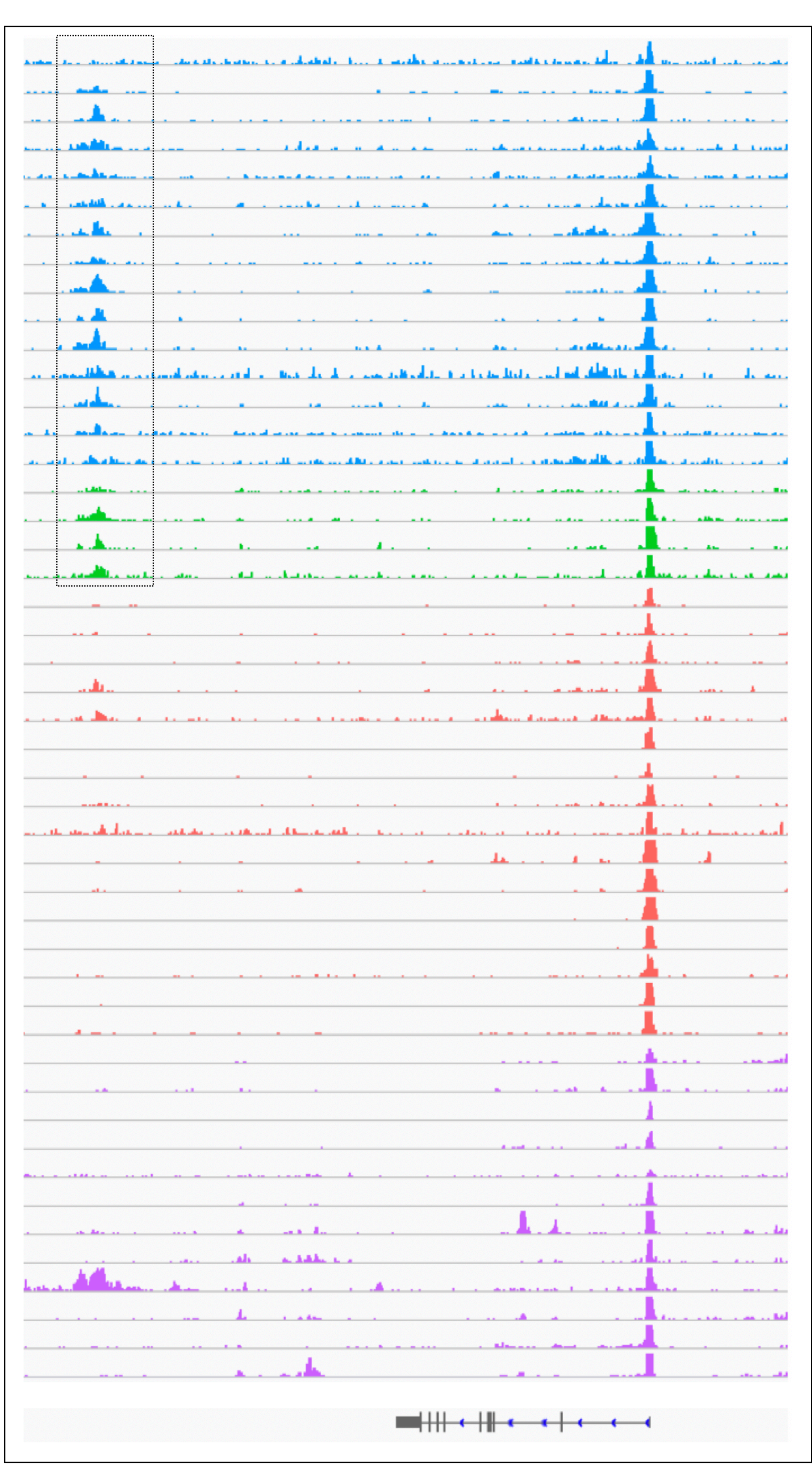

SMAD2
B

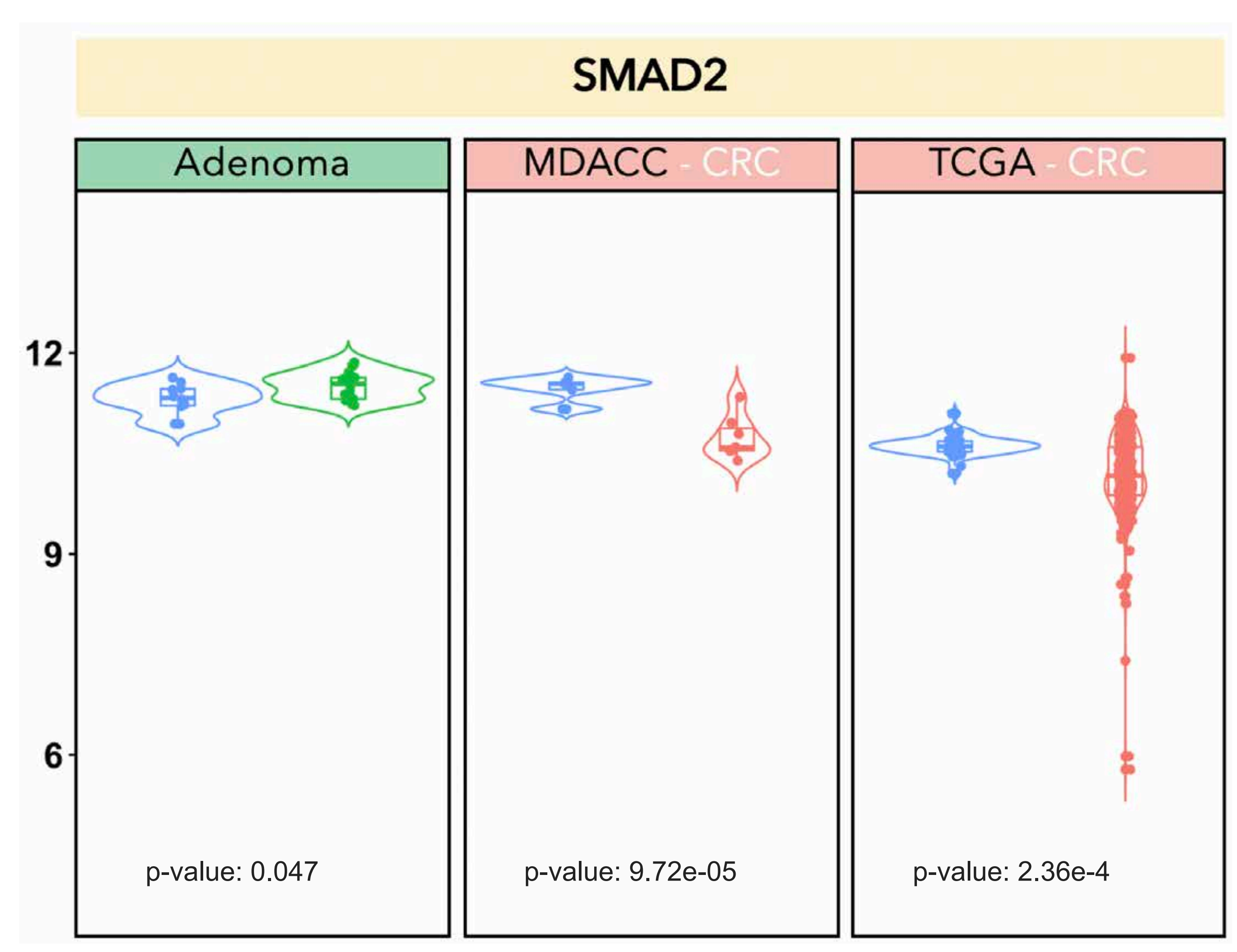

国 Normal 直 CRC 国 Adenoma

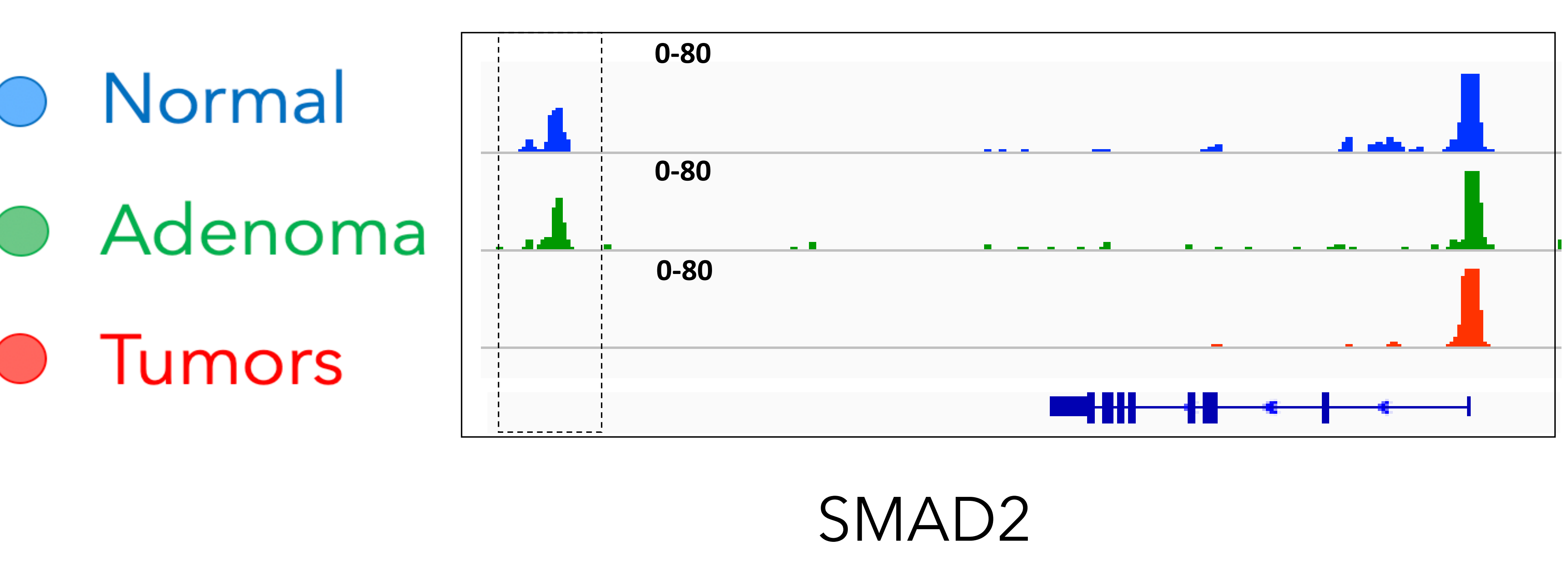

C

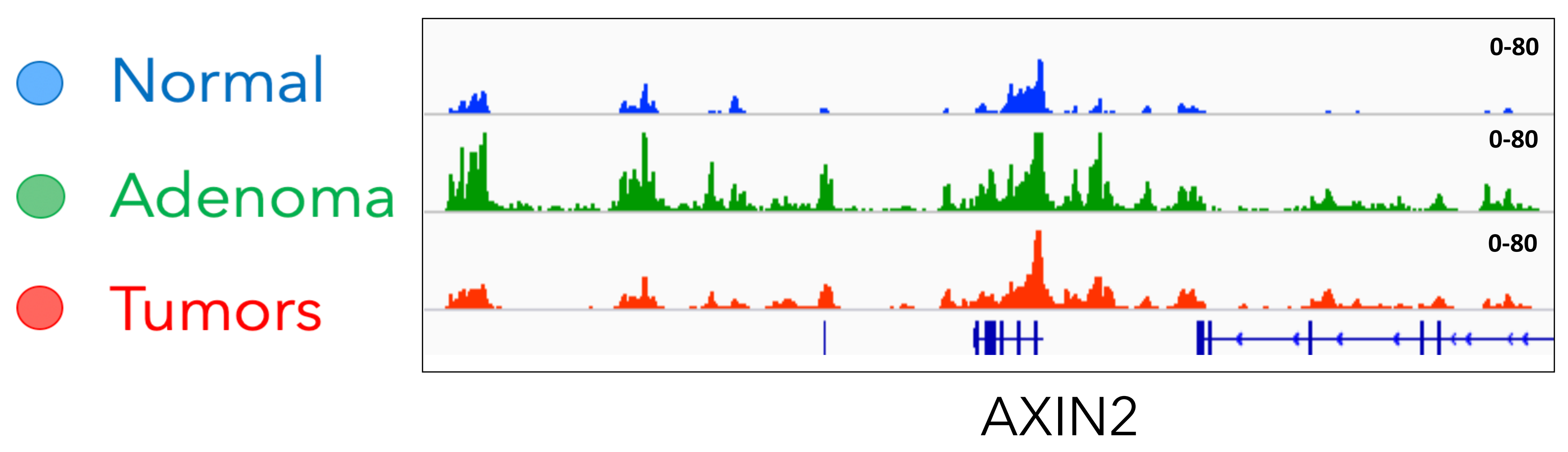

\begin{tabular}{l} 
Normal \\
Adenoma \\
Tumors \\
\hdashline \\
\hdashline
\end{tabular}

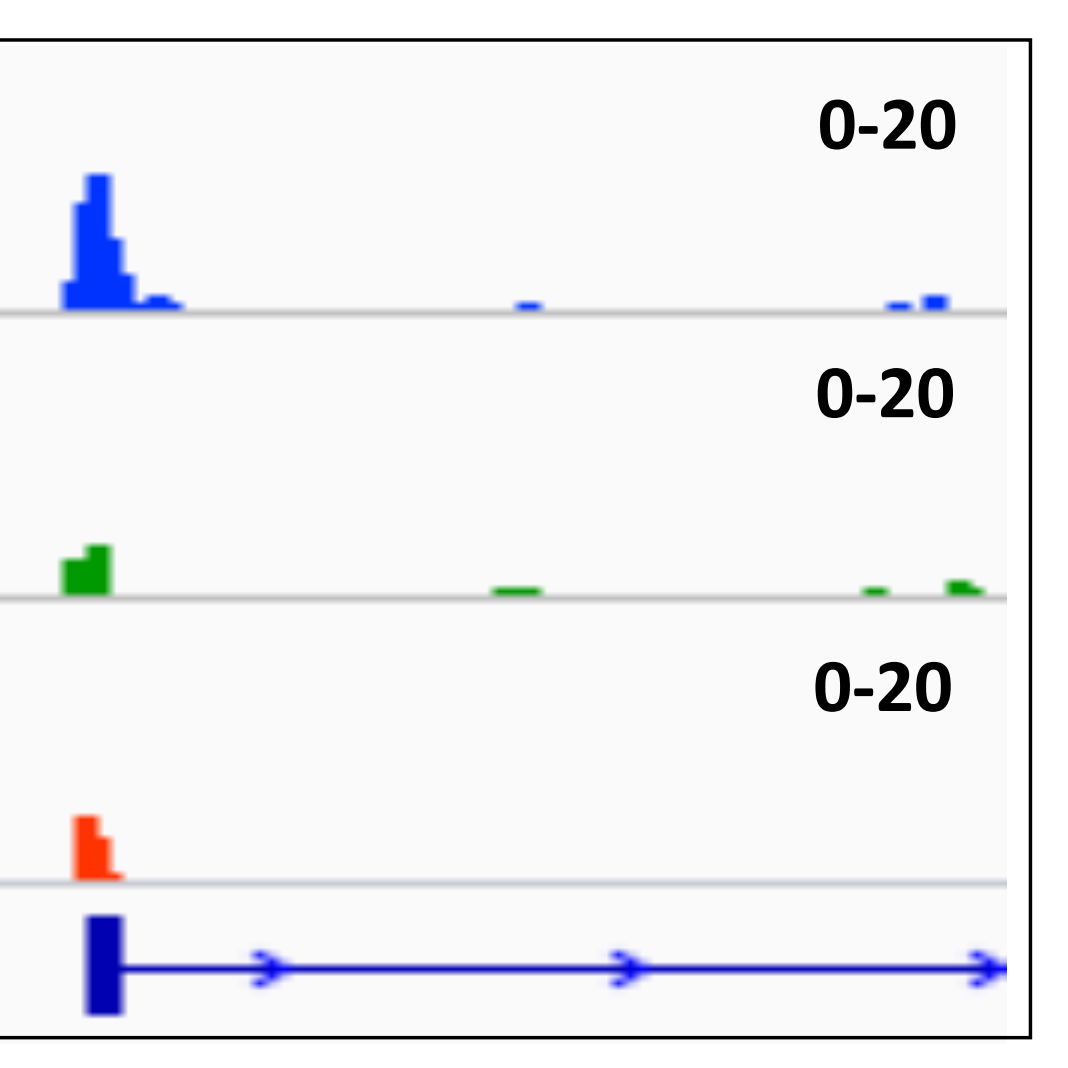

TCF7L1

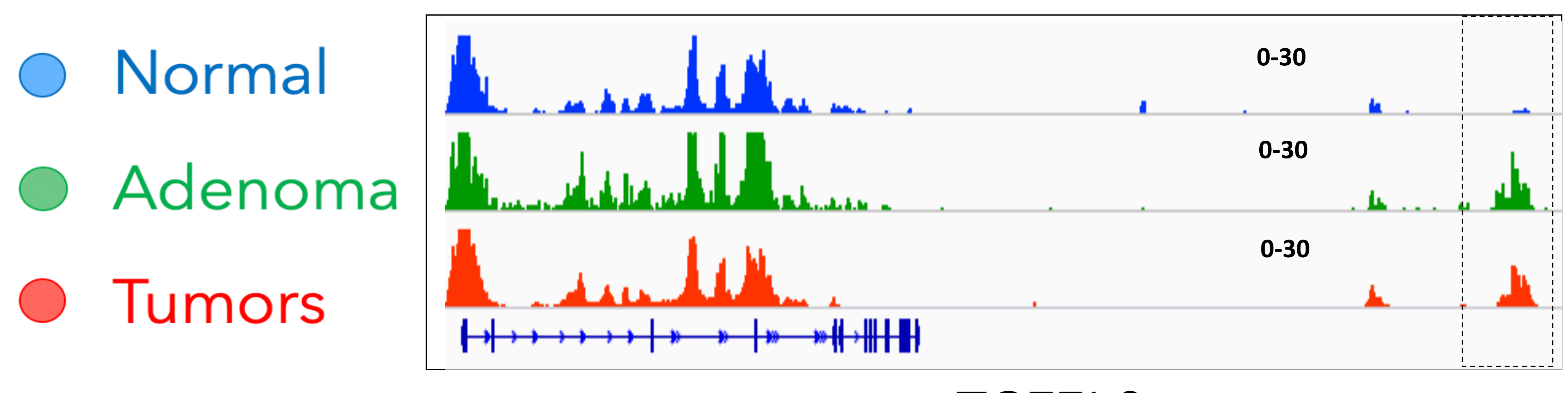

TCF7L2

\begin{tabular}{|c|c|c|c|}
\hline \multicolumn{3}{|c|}{ T- specific motifs } & \multirow{2}{*}{$\begin{array}{l}\text { p-value } \\
1.00 E-33\end{array}$} \\
\hline 1 & 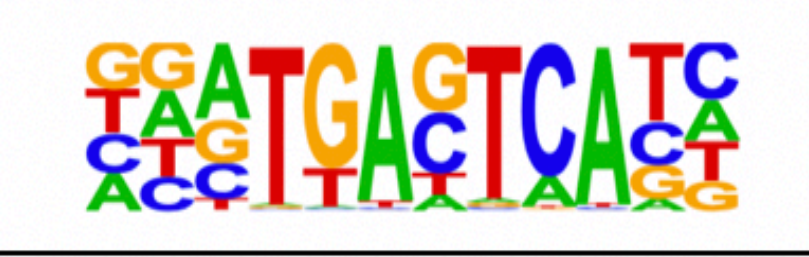 & Fra1 (bzlP) & \\
\hline 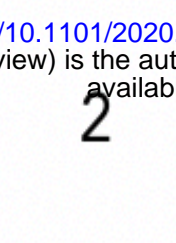 & 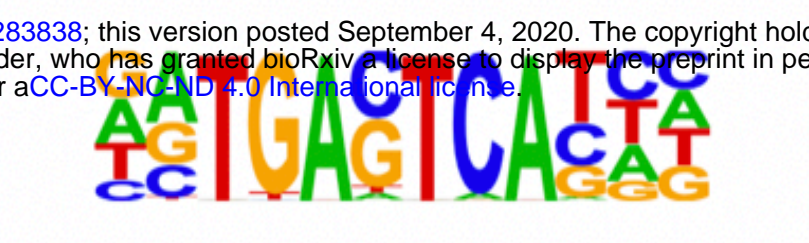 & $=4+3(6 Z 1 P)$ & $1.00 E-27$ \\
\hline 3 & AAAGTAAACA & FOXA1 (Forkhead) - LNCAP & $1.00 E-26$ \\
\hline 4 & 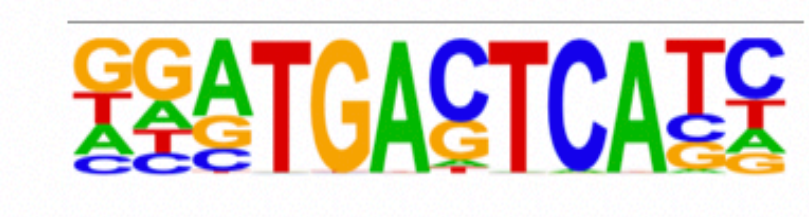 & $\operatorname{Fra2}($ bZlP) & $1.00 E-26$ \\
\hline 5 & ATGACTCATC & AP-1 (bZIPO & $1.00 E-24$ \\
\hline 6 & AAAGTAACCA & FOXA1 (Forkhead) - MCF7 & $1.00-24$ \\
\hline 7 & 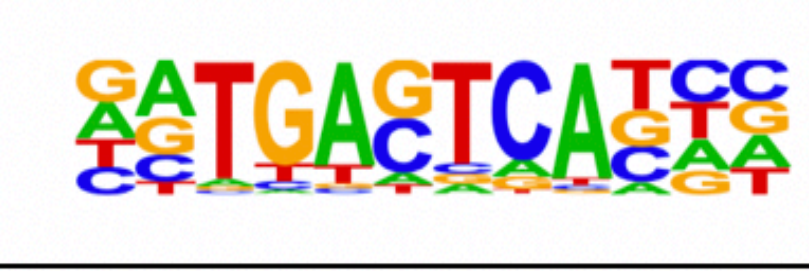 & Fosl2 (bZIP) & $1.00=23$ \\
\hline 8 & TATIACTCA & FOXM1 (Forkhead) & $1.00 E-22$ \\
\hline 9 & TATGACTCAT & BATF (GZZPPO & $1.00 E-21$ \\
\hline 10 & ACCCGAAGT & ETV4 (ETS) & $1.00-21$ \\
\hline
\end{tabular}

\begin{tabular}{|c|c|c|c|}
\hline \multicolumn{3}{|c|}{ N-specific motifs } & \multirow{2}{*}{$\begin{array}{l}\text { p-value } \\
1.00 E-03\end{array}$} \\
\hline 1 & AGCCCTAG & ZNF711 (Zf) & \\
\hline 2 & CGGTGACGTCAC & CRE (GZIP) & $1.00 E-02$ \\
\hline 3 & ITCECCGAAA & E2F (E2F) & $1.00 E-02$ \\
\hline 4 & AGGTCA合TCACCTCC & FXR (NR) & 1.00E-02 \\
\hline 5 & TEGAGTCETCE & Bapx1 (Homeobox) & $1.00 E-02$ \\
\hline 6 & AACACCCCTC & $\operatorname{LRF}(7 \mathrm{ZT}$ & $1.00 E-02$ \\
\hline 7 & CTAAAAATAG & Mefza (MADS) & $1.00 E-02$ \\
\hline 8 & TECATTCCAG & TEAD3 (TEA) & $1.00 E-02$ \\
\hline 9 & 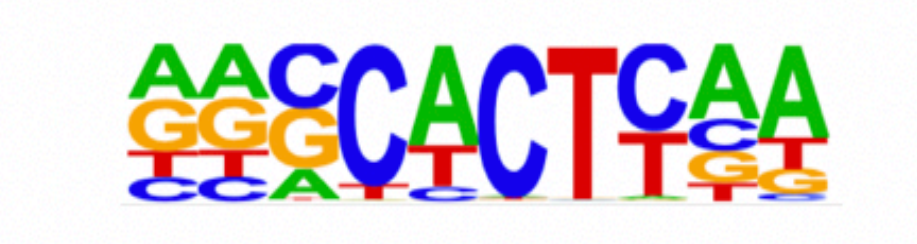 & NKx2.5 (Homeobox) & $1.00 E-02$ \\
\hline 10 & AACCACTTAA & NK×3.1 (Homeobox) & $1.00 E-02$ \\
\hline
\end{tabular}

\begin{tabular}{|c|c|c|c|}
\hline & A-spe & fic motifs & p-value \\
\hline 1 & 'CCTTGTTCC & Sox4 (HMG) & $1.00 E-02$ \\
\hline 2 & हैACACACGTGG & n-Myc (bHLLH) & $1.00 E-02$ \\
\hline 3 & 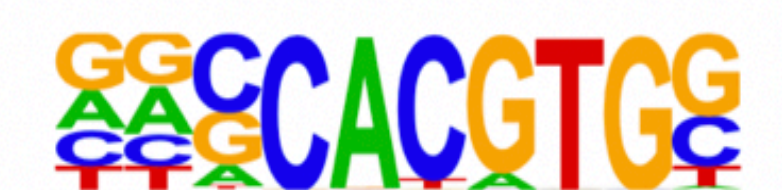 & $c-M y c(b H H L H)$ & $1.00 E=02$ \\
\hline 4 & ACCCCTAG & ZNF711 (Z7) & $1.00 E-02$ \\
\hline
\end{tabular}


Figure $\$ 4$
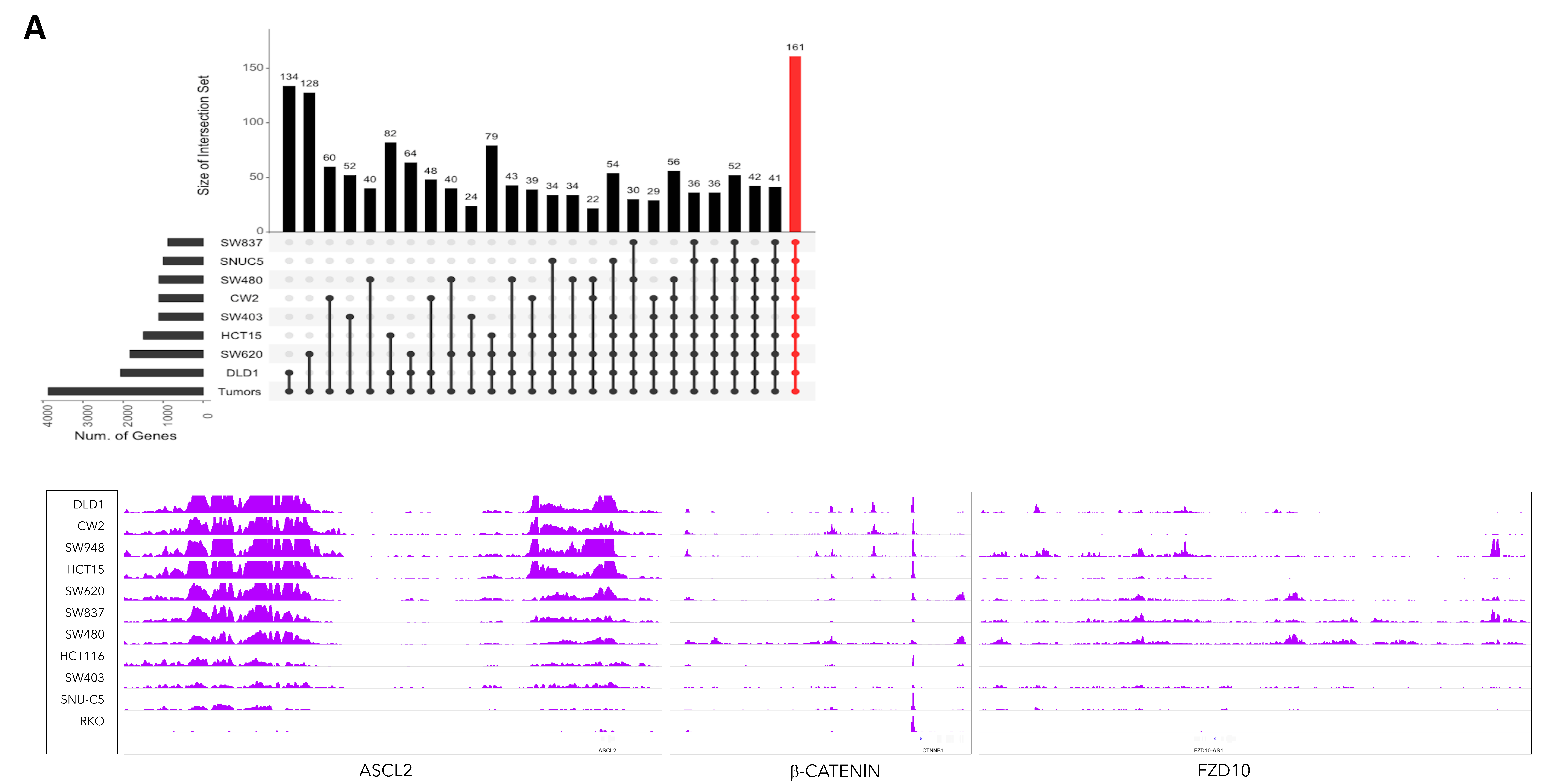

B
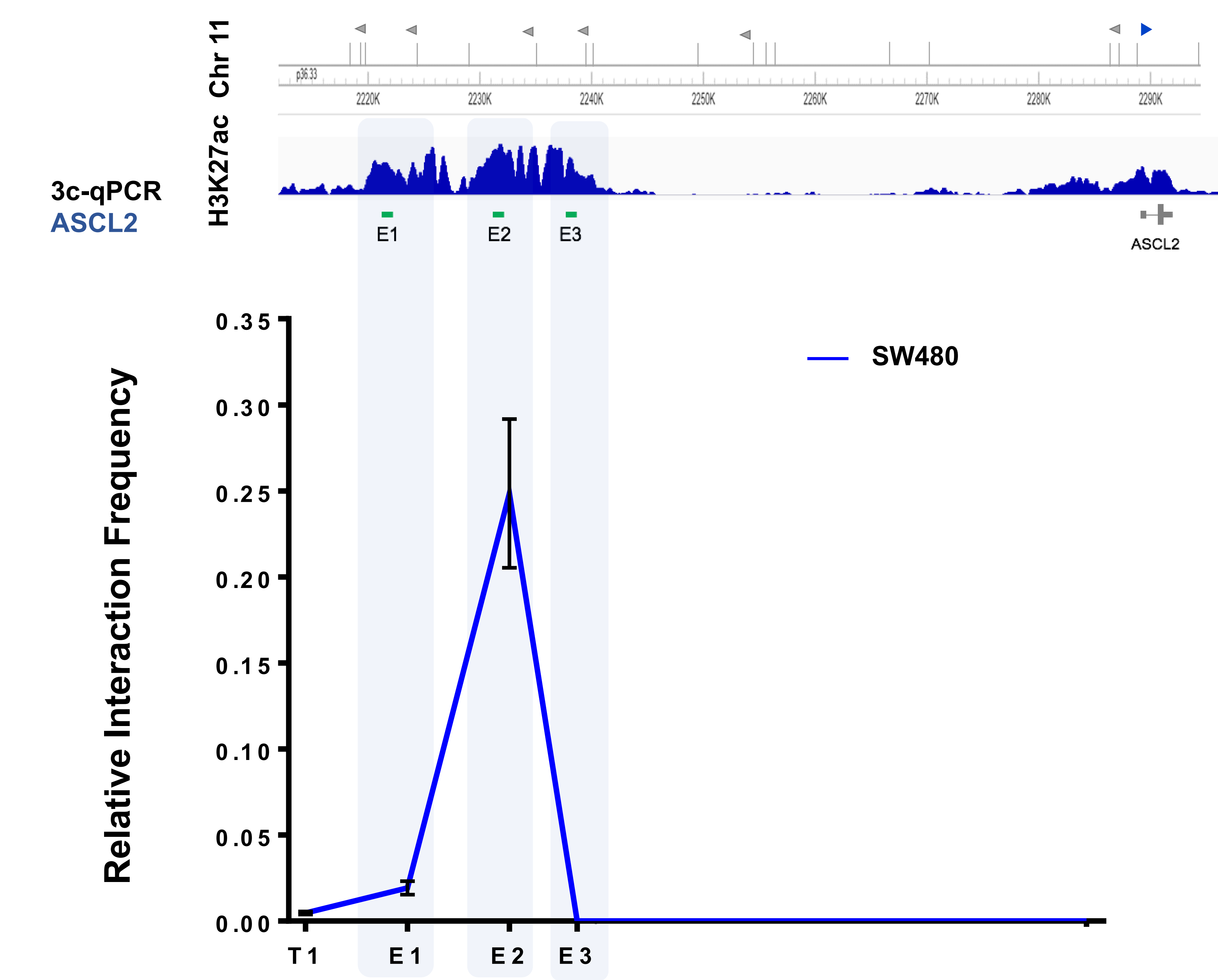

D

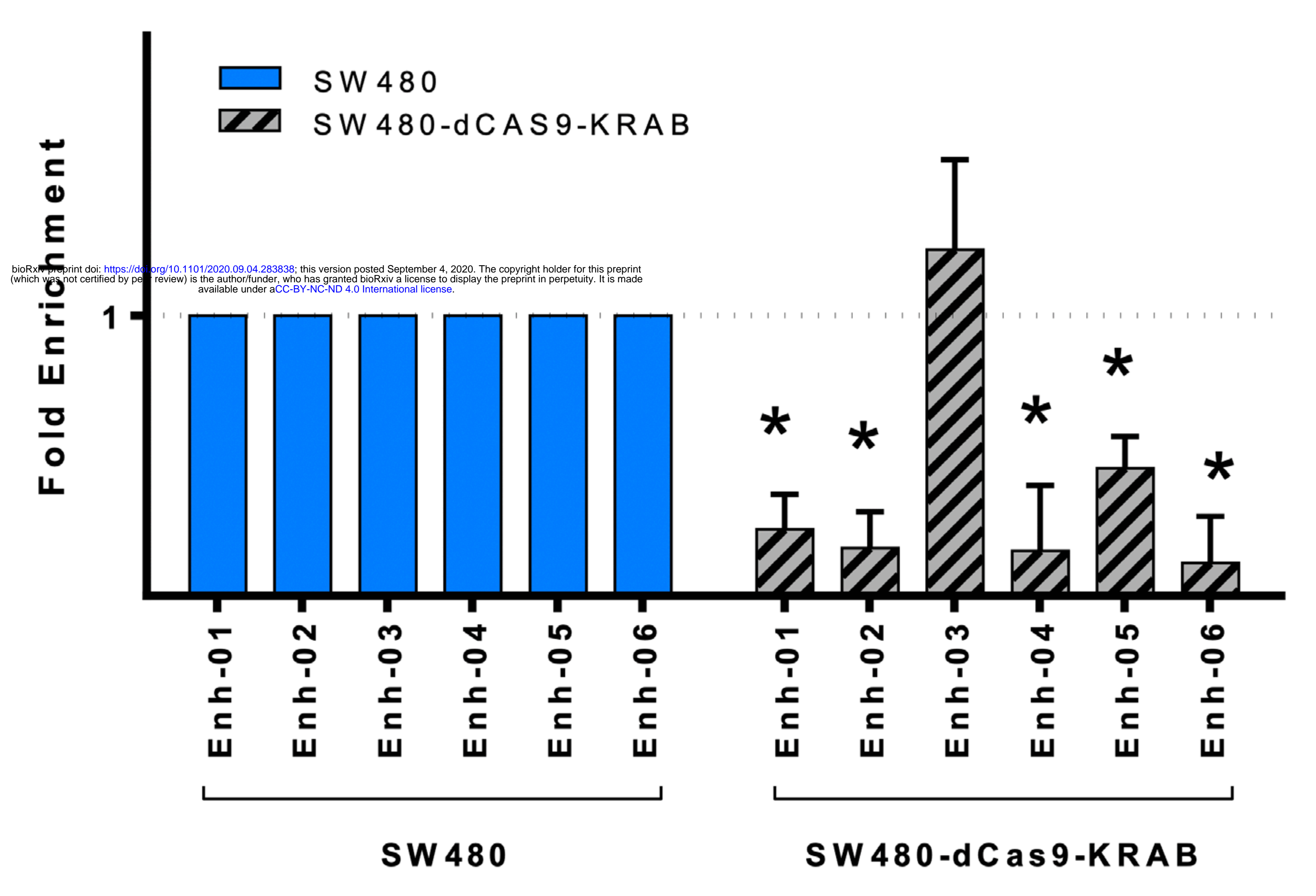

E
C

\section{Chr12}
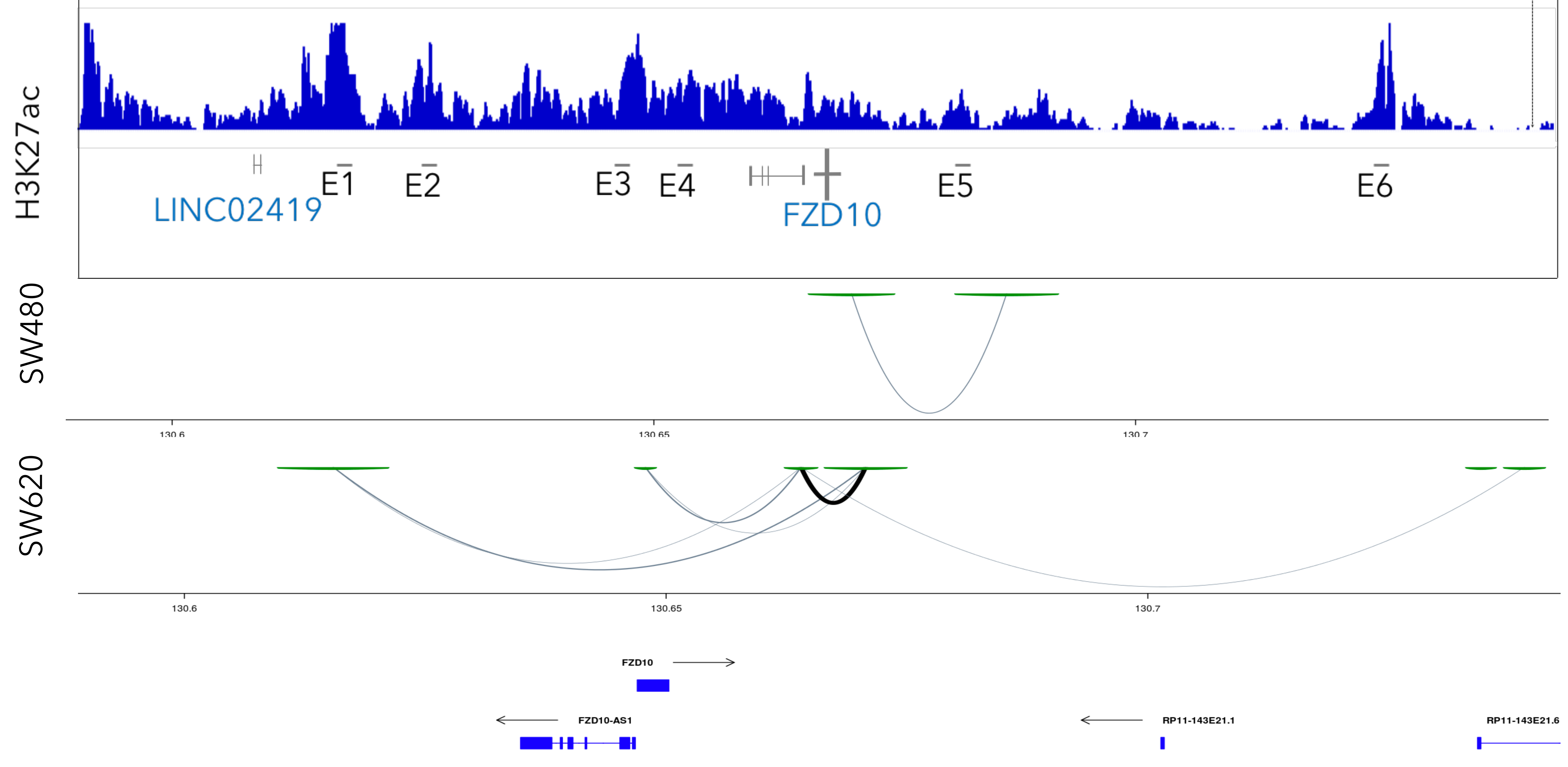

F

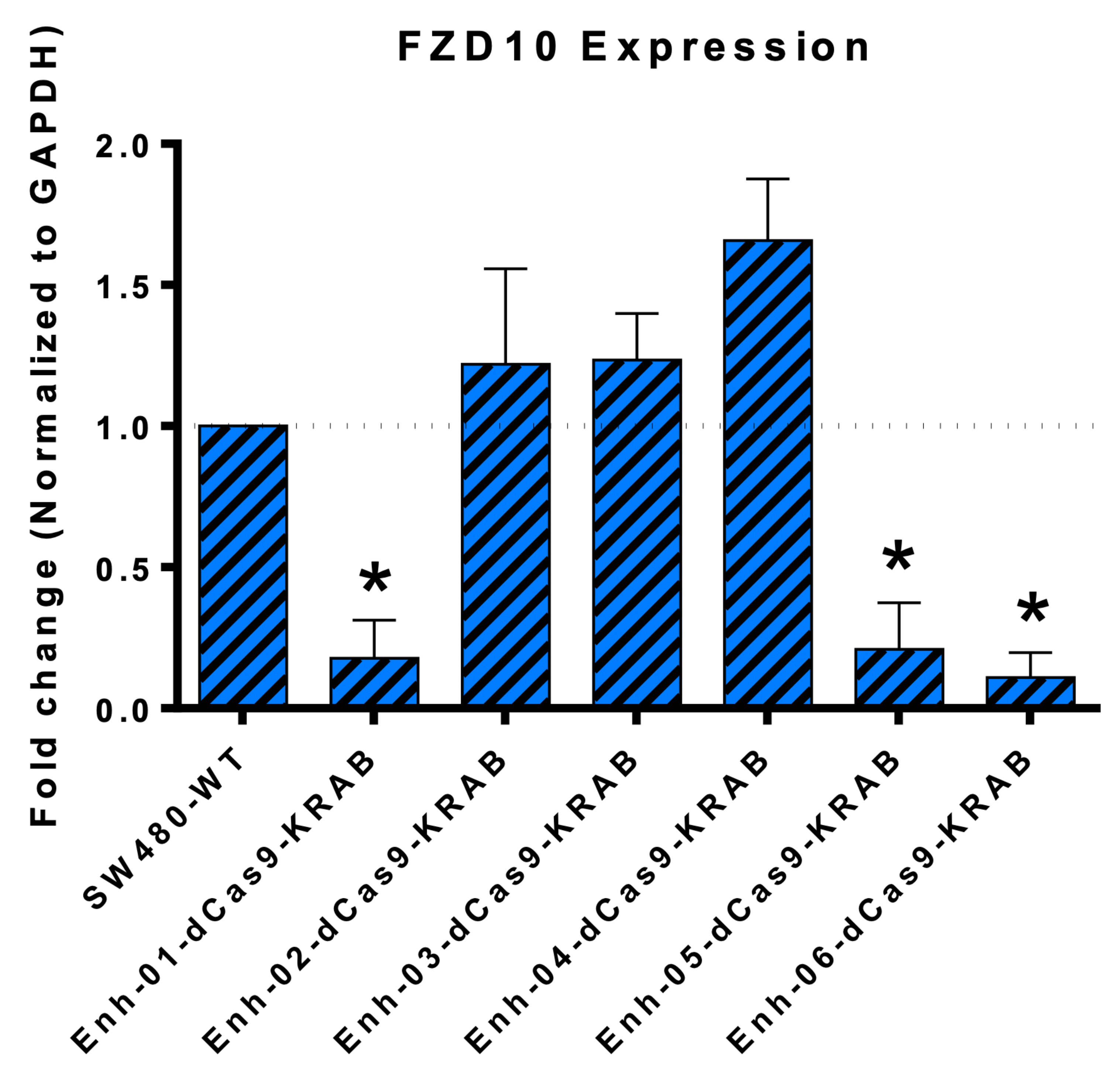


Figure S5

A<smiles>COc1cc(OC)c2c(=O)[nH]c(-c3cc(C)c(OCCO)c(C)c3)nc2c1</smiles><smiles>CCN(C)CCC(C)C(=O)OCCOc1c(C)cc(-c2nc3cc(OC)cc(OC)c3c(=O)[nH]2)cc1C</smiles>
$\mathrm{O} \underset{\mathrm{NH}_{2}}{ }$<smiles>CC(=O)Nc1ccc(COC(=O)COC(C)=O)cc1</smiles><smiles>CNCCCC(C)NC(=O)[C@H](NC(=O)CCCCCN1C(=O)C=CC1=O)C(C)C</smiles><smiles>CNCCN(C)C(=O)OCCOc1c(C)cc(-c2nc3cc(OC)cc(OC)c3c(=O)[nH]2)cc1C</smiles>

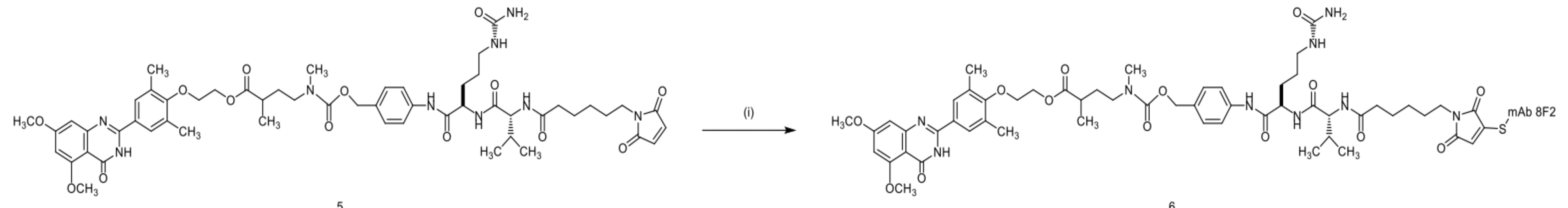

B

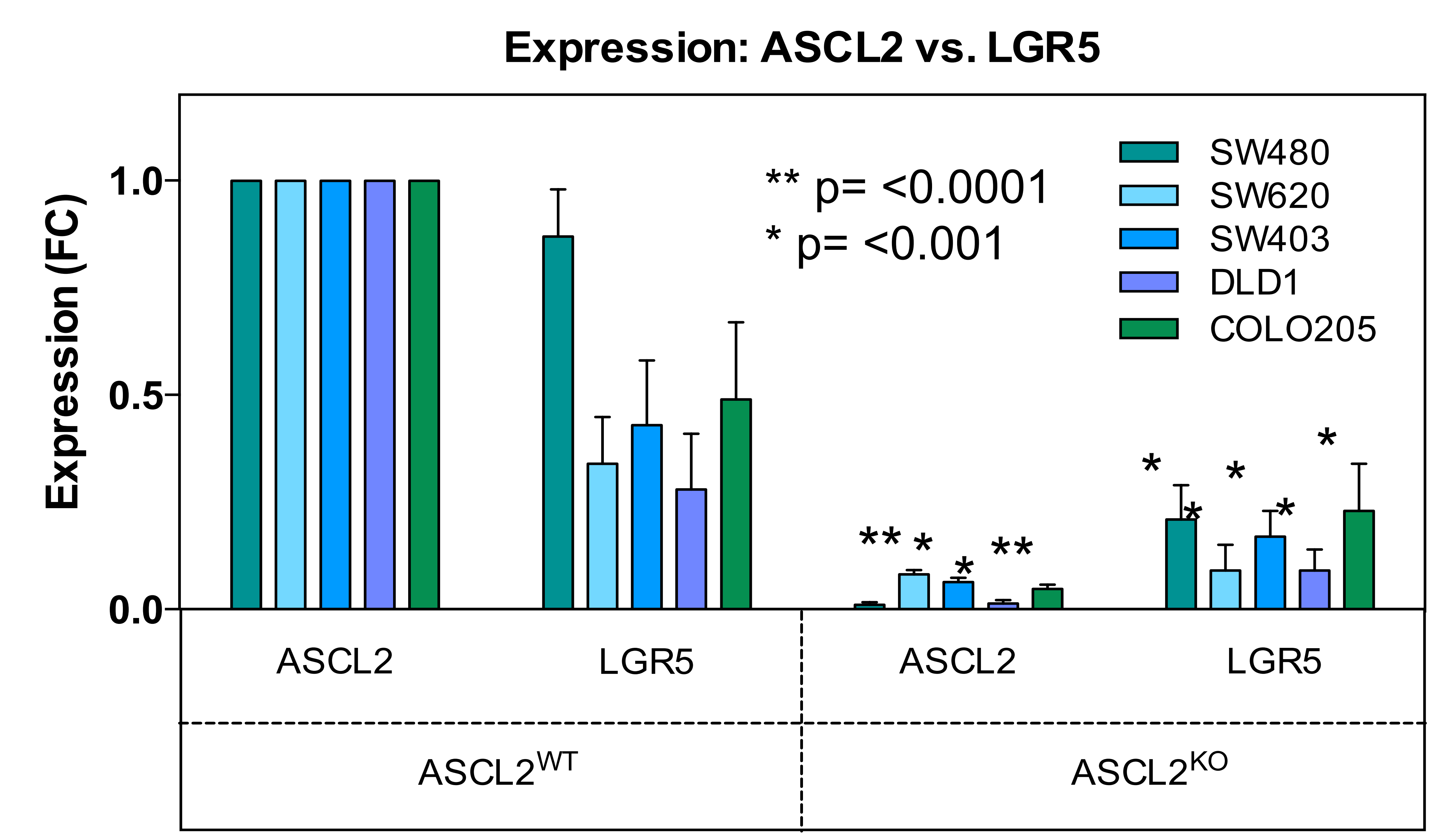

D

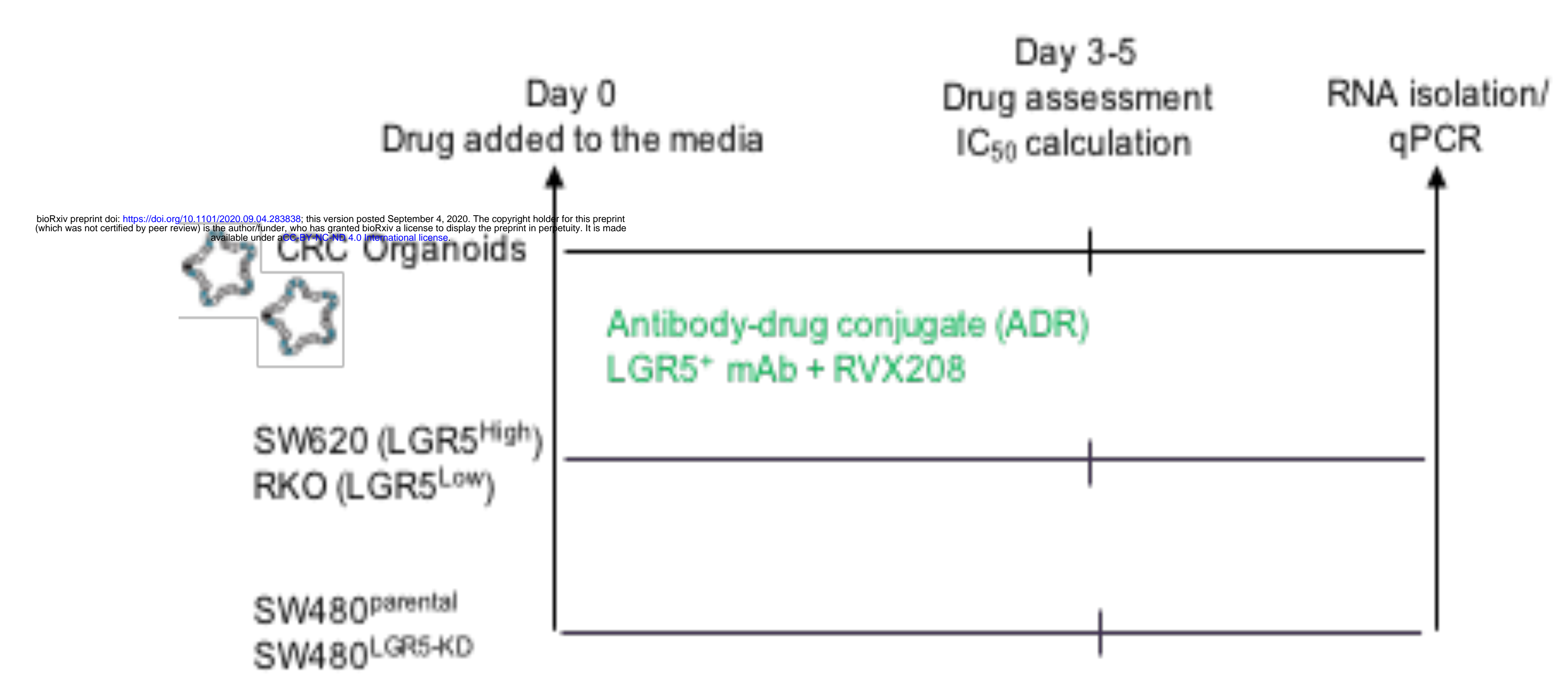

\section{C}

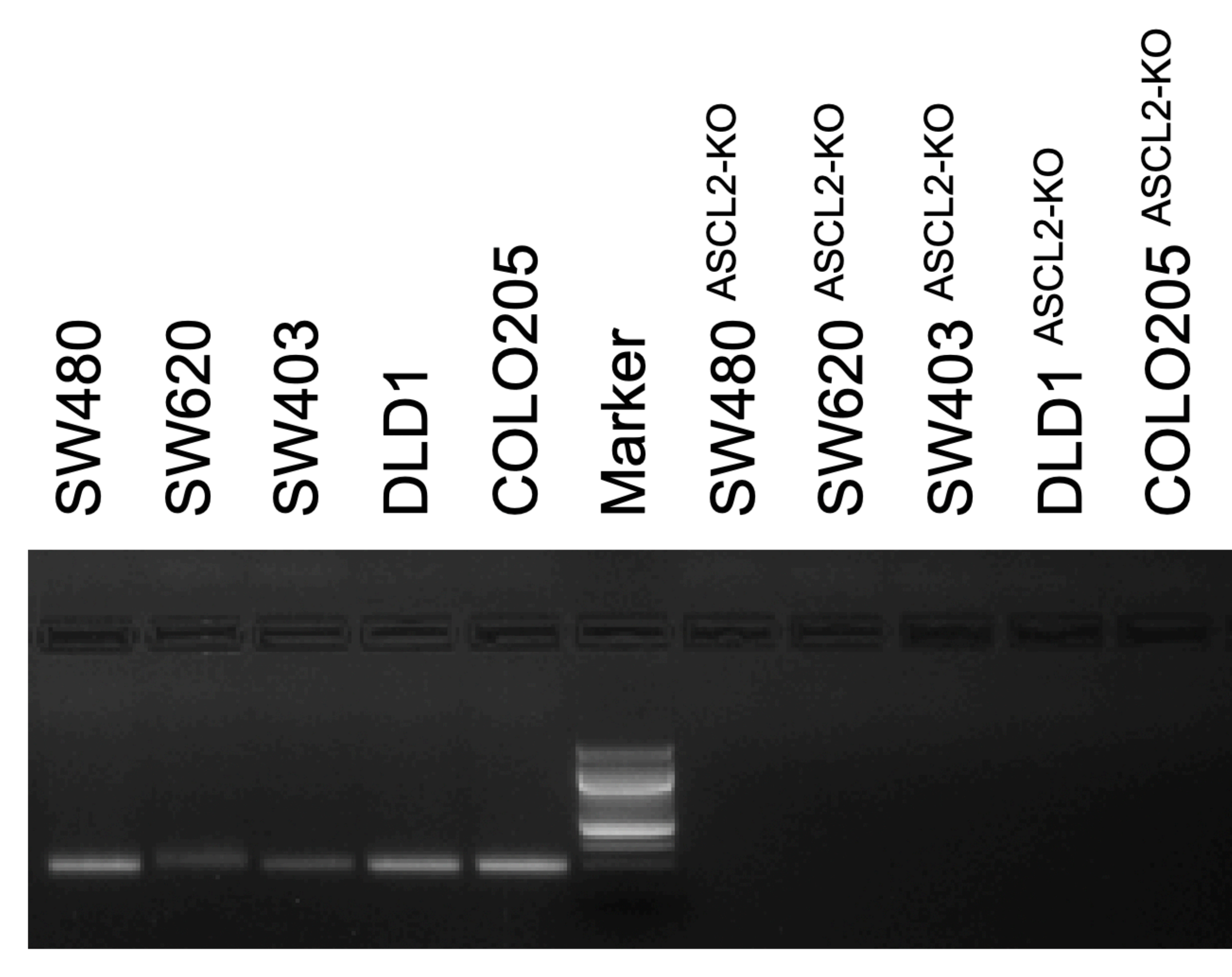

E

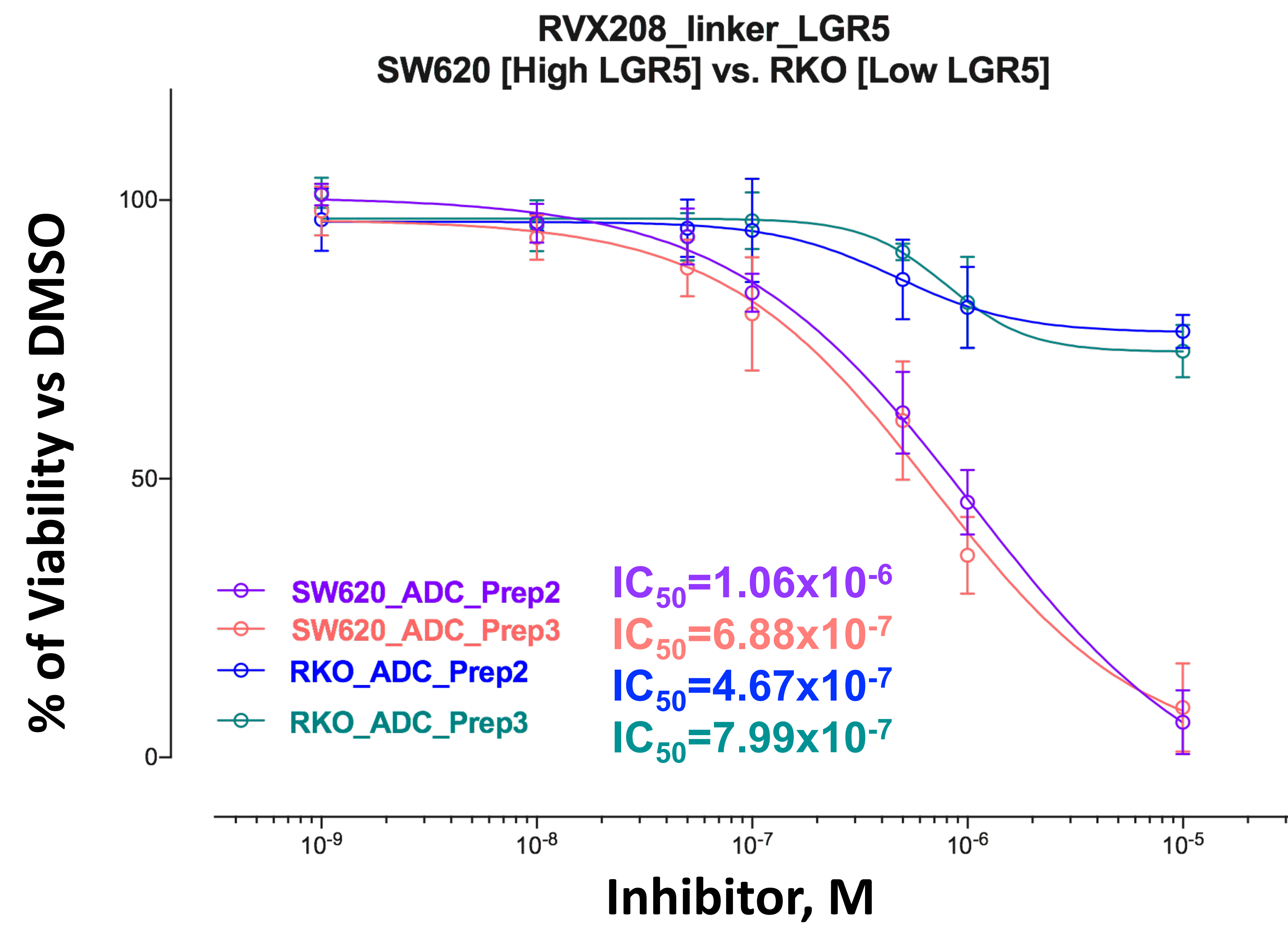


Figure S6

A

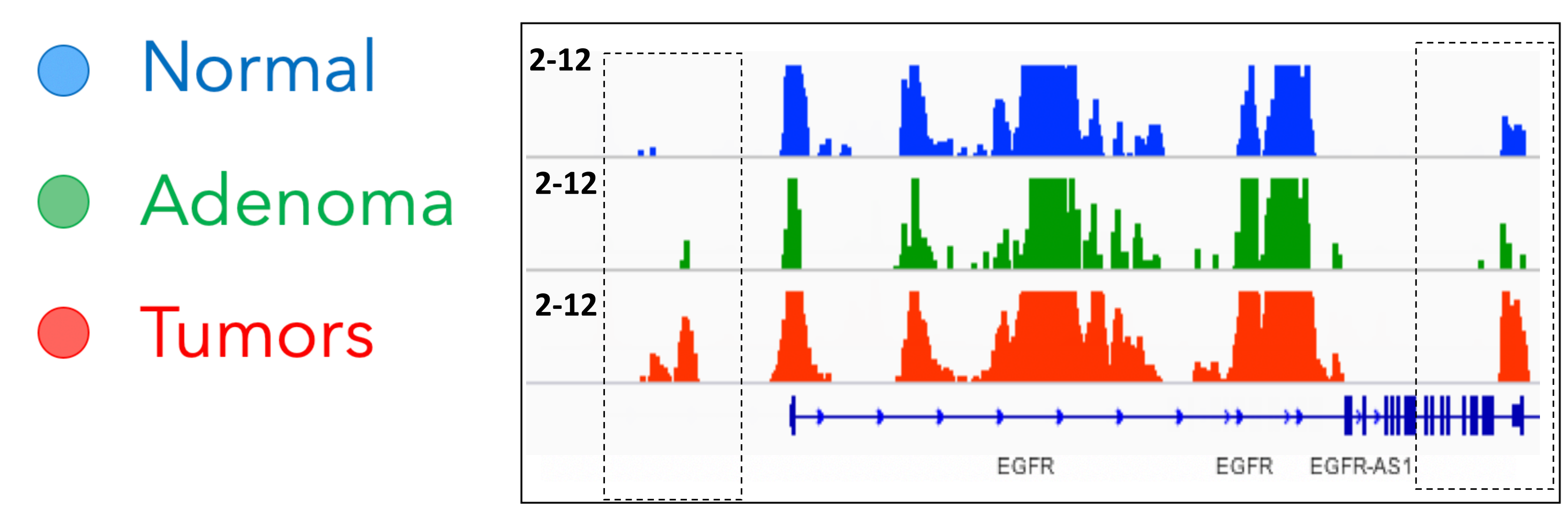

EGFR

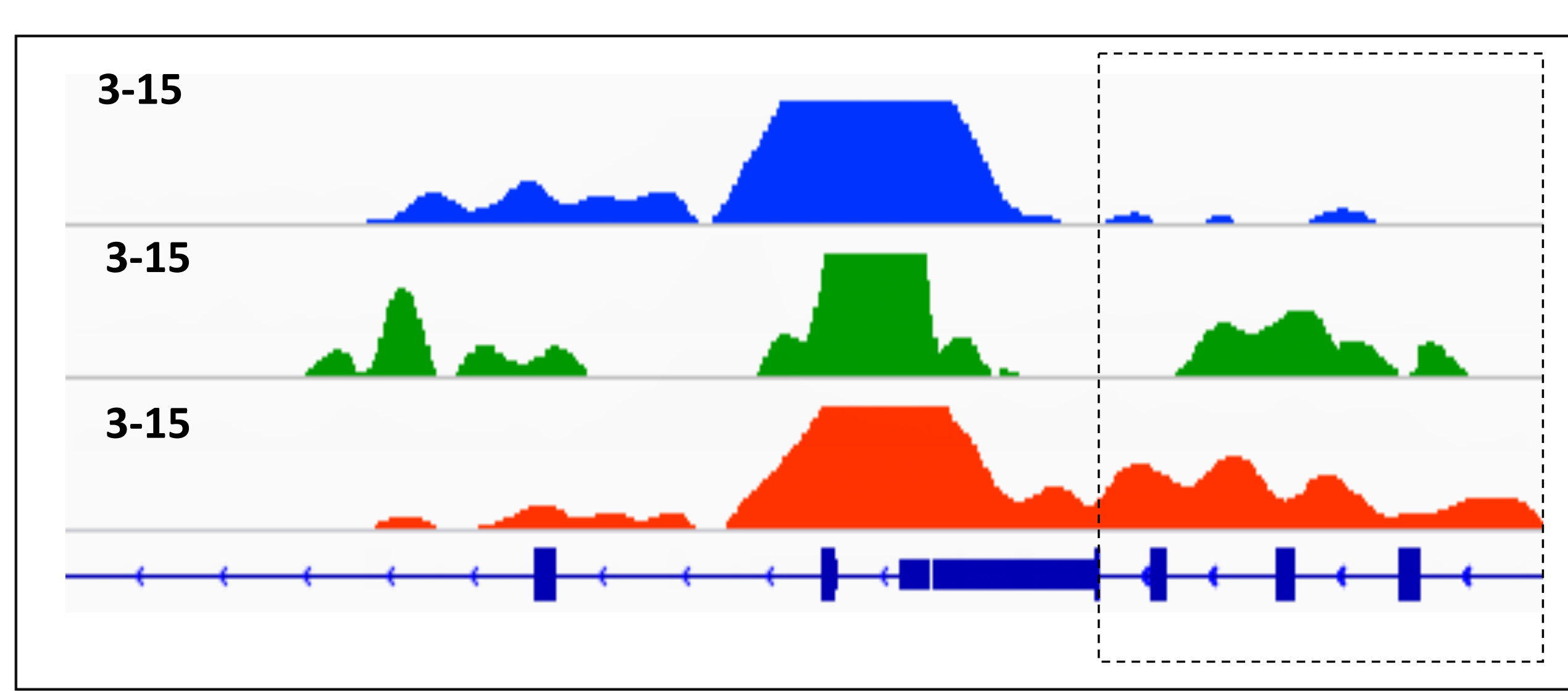

NRAS

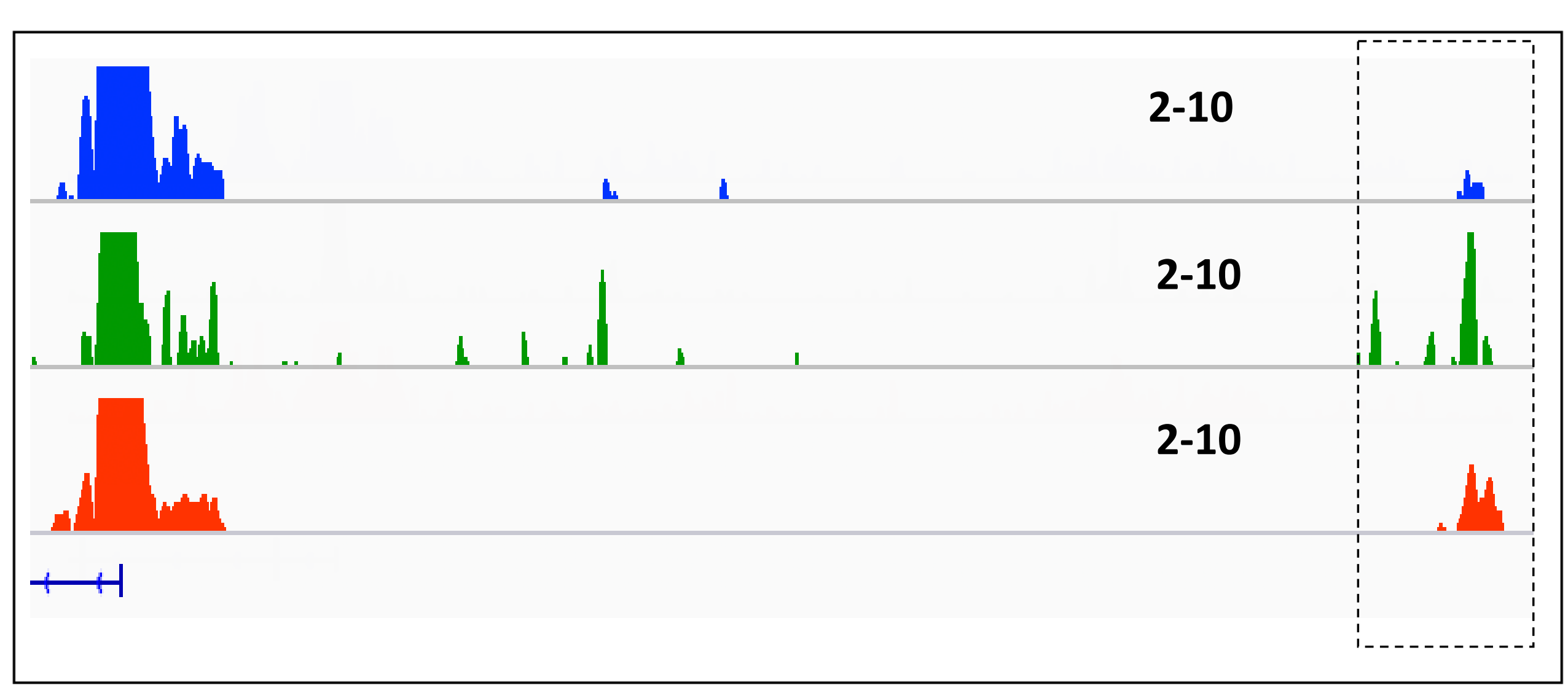

KRAS

- Normal

- Adenoma

- Tumors

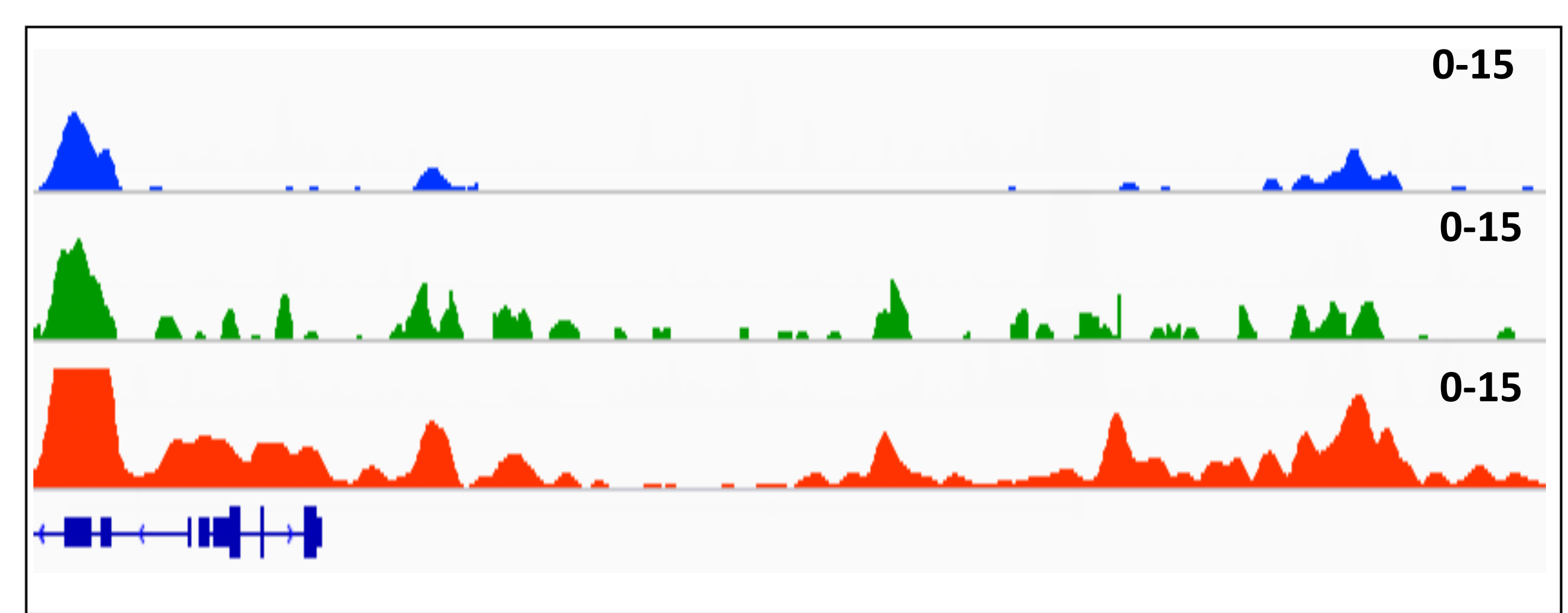

FGF21

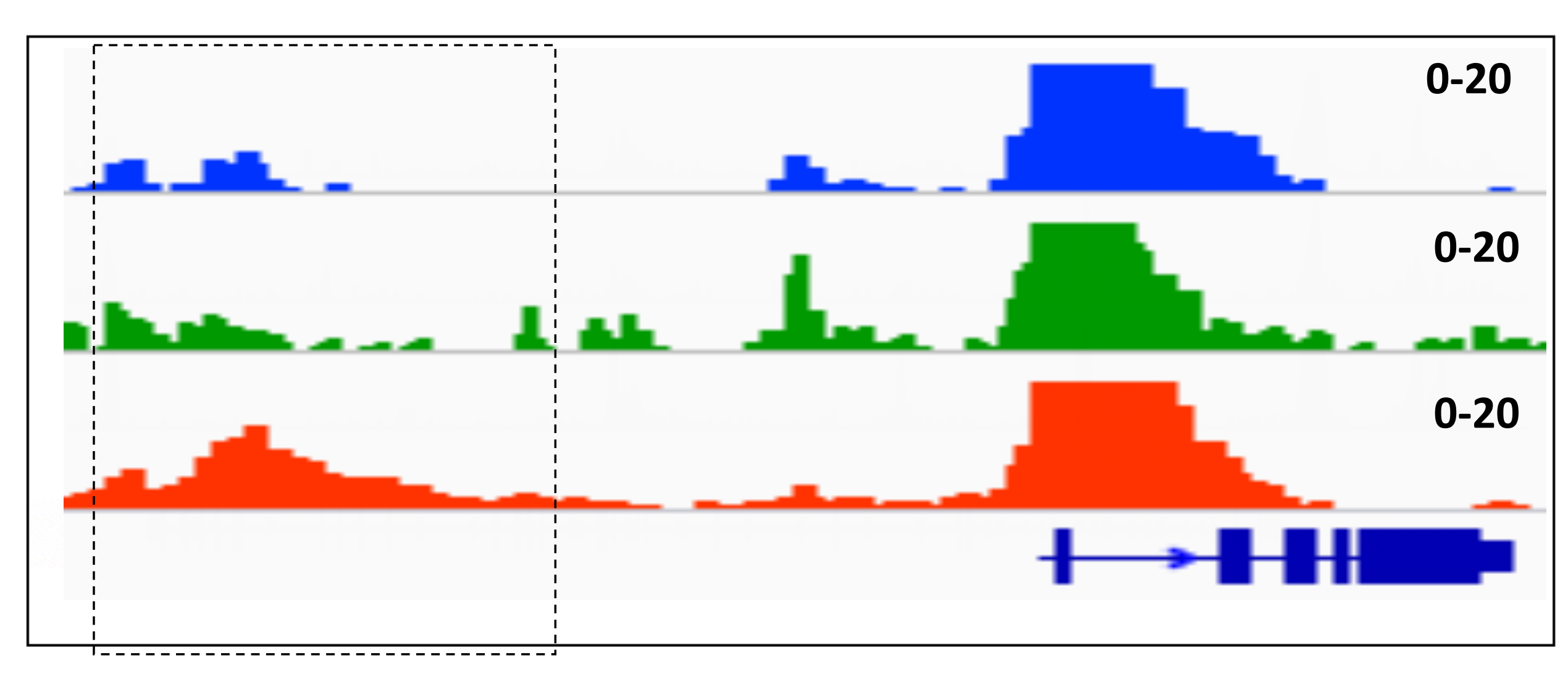

FGFR3

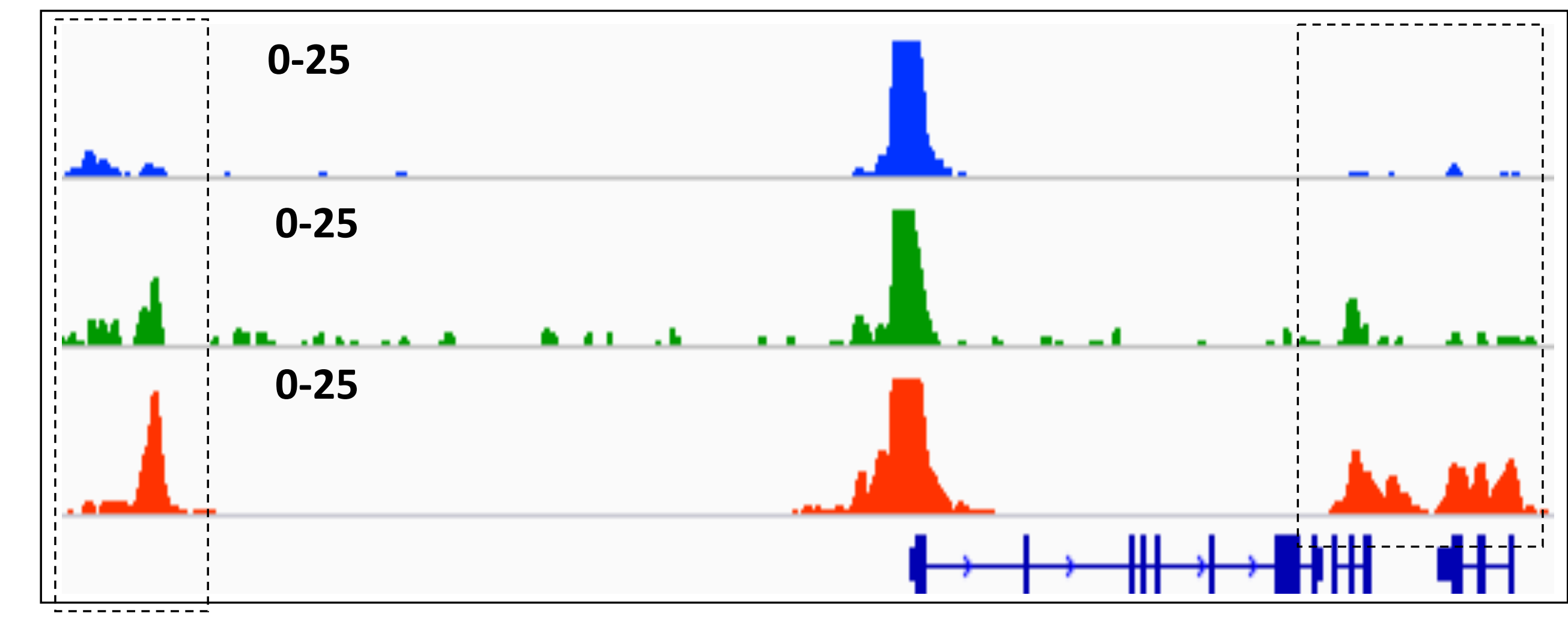

MAP3K10

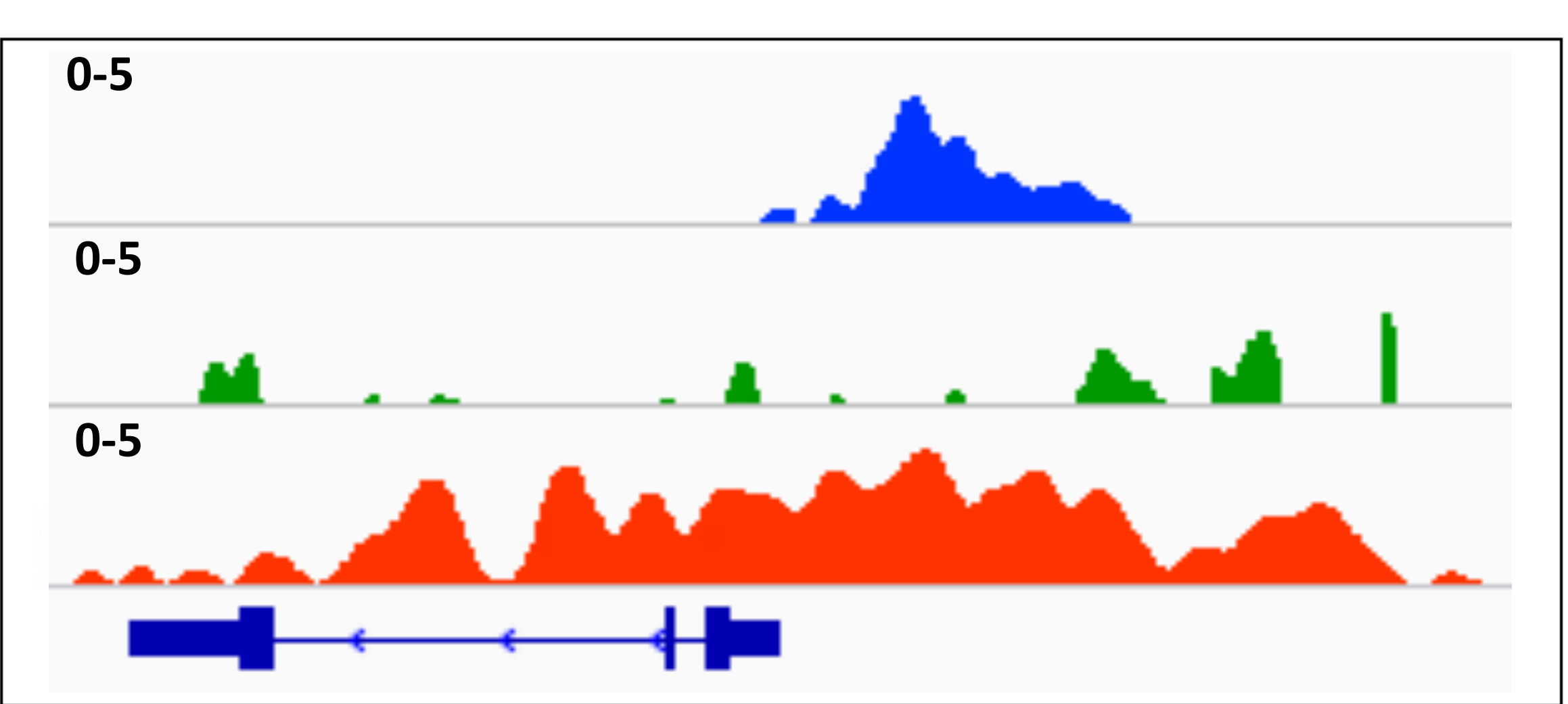

FGF19

B

\begin{tabular}{|c|c|c|c|}
\hline Model & analyzed & $p$ value & $p$ value summary \\
\hline \multirow[t]{5}{*}{ C1138 } & Ctrl vs Tram & $<0.0001$ & $* * * *$ \\
\hline & Ctrl vs iBET & 0.0571 & $\mathrm{~ns}$ \\
\hline & Ctrl vs Combo & $<0.0001$ & $* * * *$ \\
\hline & Tram VS Combo & 0.1413 & $\mathrm{~ns}$ \\
\hline & iBET VS Combo & 0.0004 & $* * *$ \\
\hline \multirow[t]{5}{*}{ C1138 } & Ctrl vs Bini & $<0.0001$ & $* * * *$ \\
\hline & Ctrl vs iBET & 0.0571 & $\mathrm{~ns}$ \\
\hline & Ctrl vs Combo & $<0.0001$ & $* * * *$ \\
\hline & Bini VS Combo & 0.0804 & $\mathrm{~ns}$ \\
\hline & iBET VS Combo & 0.0034 & ** \\
\hline \multirow[t]{5}{*}{ F3053 } & Ctrl vs Tram & 0.0119 & $*$ \\
\hline & Ctrl vs iBET & 0.2103 & $\mathrm{~ns}$ \\
\hline & Ctrl vs Combo & 0.0003 & $* * *$ \\
\hline & Tram VS Combo & $<0.0001$ & $* * * *$ \\
\hline & iBET VS Combo & 0.0003 & $* * *$ \\
\hline \multirow[t]{5}{*}{ F3053 } & Ctrl vs Bini & 0.0105 & $*$ \\
\hline & Ctrl vs iBET & 0.2103 & $\mathrm{~ns}$ \\
\hline & Ctrl vs Combo & 0.0034 & ** \\
\hline & Bini VS Combo & 0.0124 & $*$ \\
\hline & iBET VS Combo & 0.0056 & $* *$ \\
\hline \multirow[t]{5}{*}{ F3008 } & Ctrl vs Tram & 0.0143 & ${ }^{*}$ \\
\hline & Ctrl vs iBET & 0.5908 & $\mathrm{~ns}$ \\
\hline & Ctrl vs Combo & 0.0051 & $* *$ \\
\hline & Tram VS Combo & 0.0113 & $*$ \\
\hline & iBET VS Combo & 0.0239 & $*$ \\
\hline \multirow[t]{4}{*}{ F3008 } & Ctrl vs Bini & 0.0072 & $* *$ \\
\hline & Ctrl vs iBET & 0.5908 & ns \\
\hline & Ctrl vs Combo & 0.0002 & $* * *$ \\
\hline & Bini VS Combo & 0.0131 & $*$ \\
\hline \multirow{6}{*}{\begin{tabular}{|l} 
B8131 \\
\end{tabular}} & TBEFF'V Combo & 0.004 & $* *$ \\
\hline & Ctrl vs Tram & $<0.0001$ & $* * * *$ \\
\hline & Ctrl vs iBET & $<0.0001$ & $* * * *$ \\
\hline & Ctrl vs Combo & $<0.0001$ & $* * * *$ \\
\hline & Tram VS Combo & 0.0082 & $* *$ \\
\hline & iBET VS Combo & 0.0003 & $* * *$ \\
\hline \multirow[t]{5}{*}{ B8131 } & Ctrl vs Bini & 0.0074 & ** \\
\hline & Ctrl vs iBET & 0.02 & $*$ \\
\hline & Ctrl vs Combo & 0.0019 & $* *$ \\
\hline & Bini VS Combo & 0.0013 & $* *$ \\
\hline & iBET VS Combo & 0.0001 & ** \\
\hline
\end{tabular}




\section{Figure S7}

A
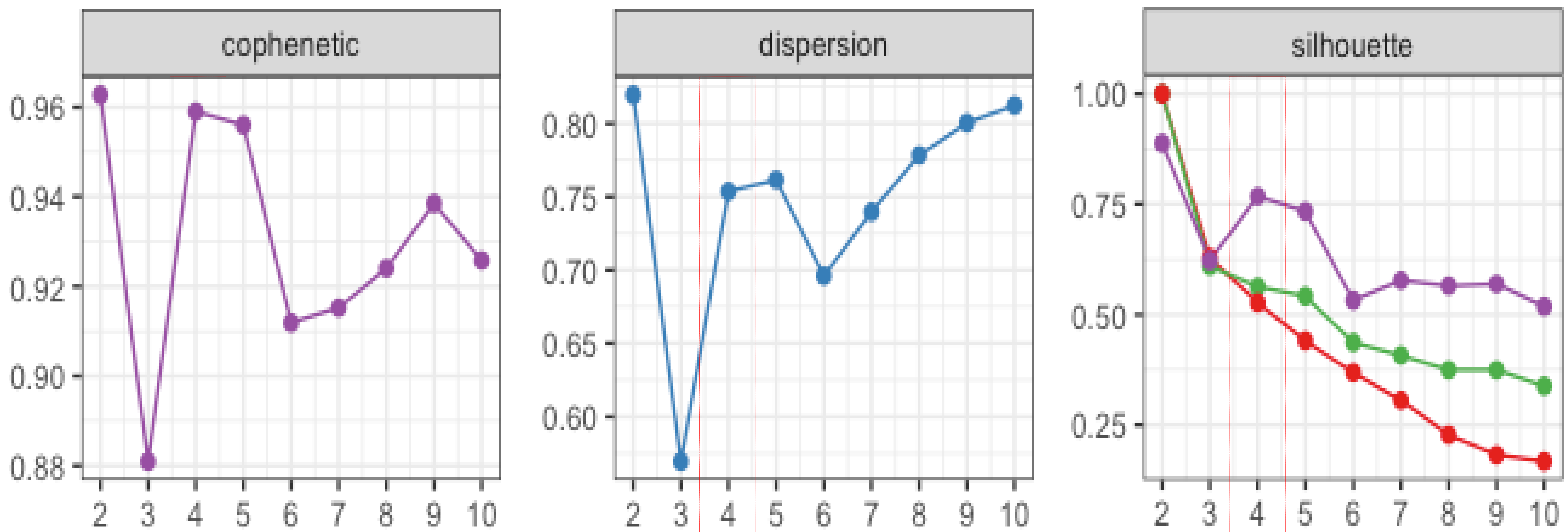

Measure type

- Basis

- - Best fit

- Coefficients

- Consensus

B
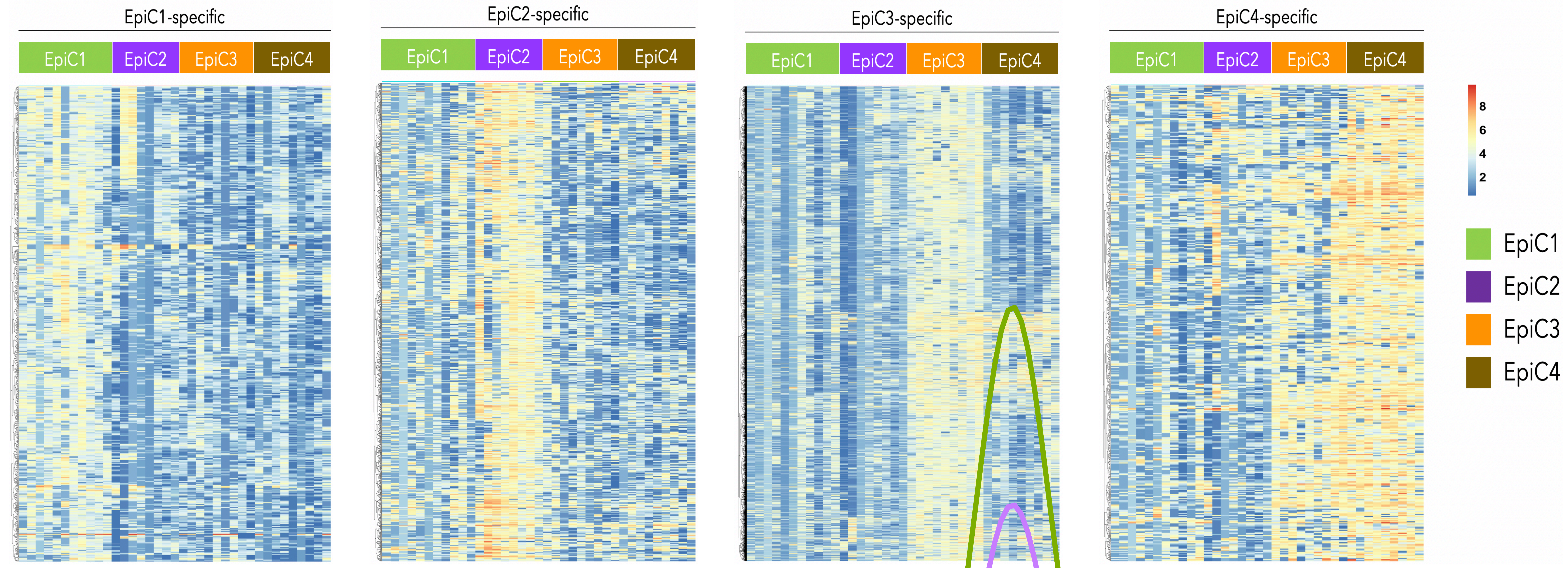

C

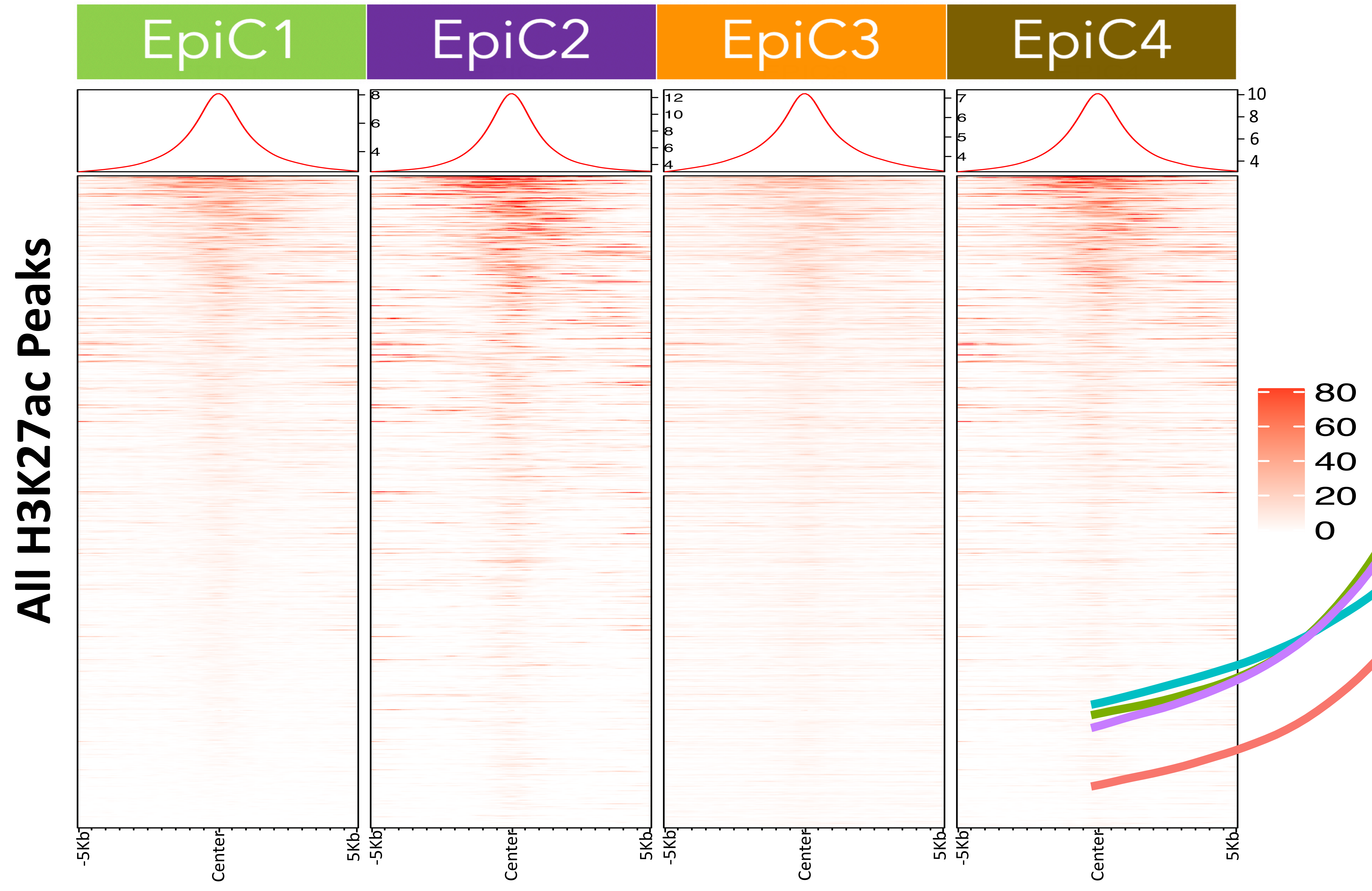

D

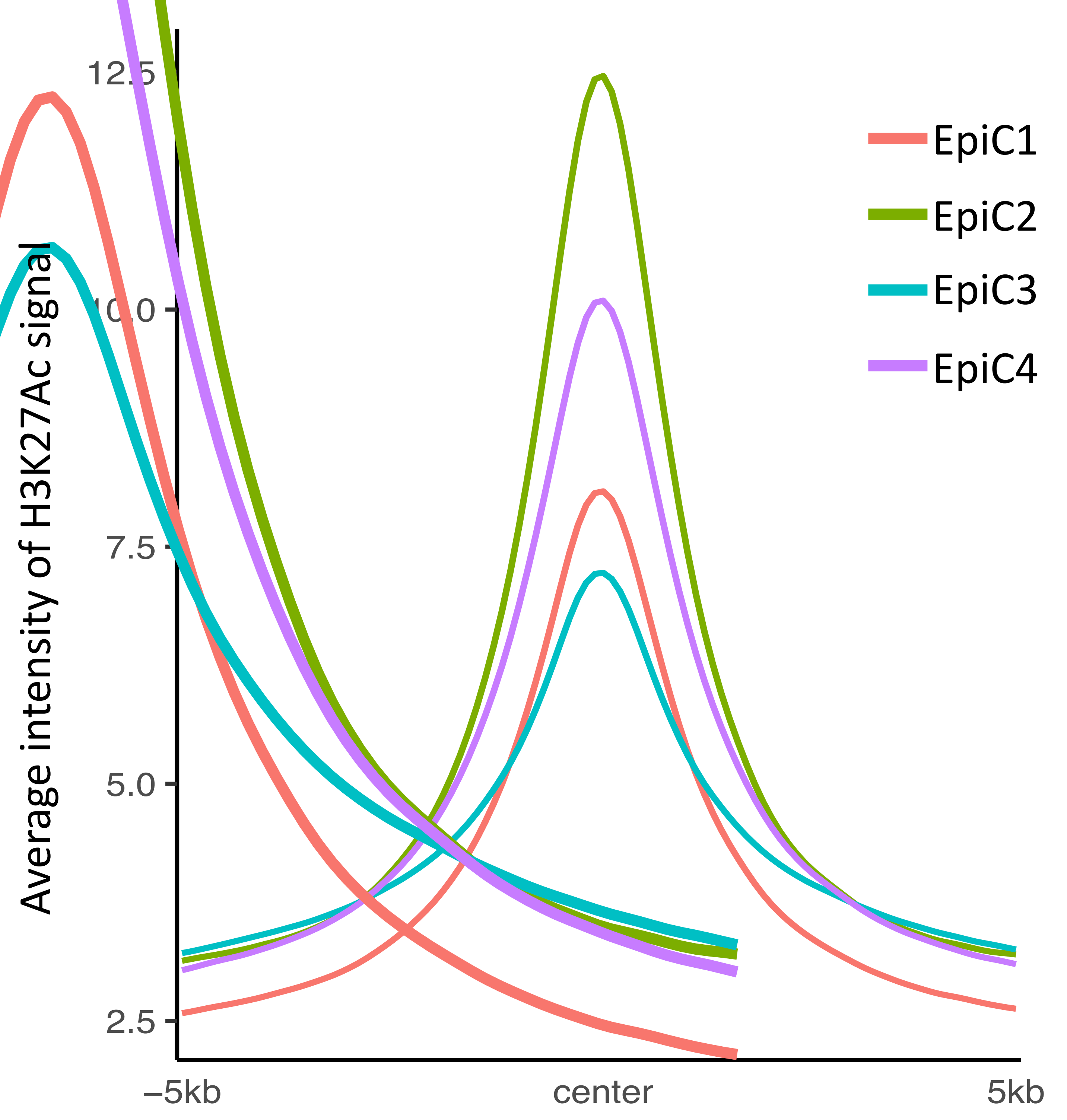

E

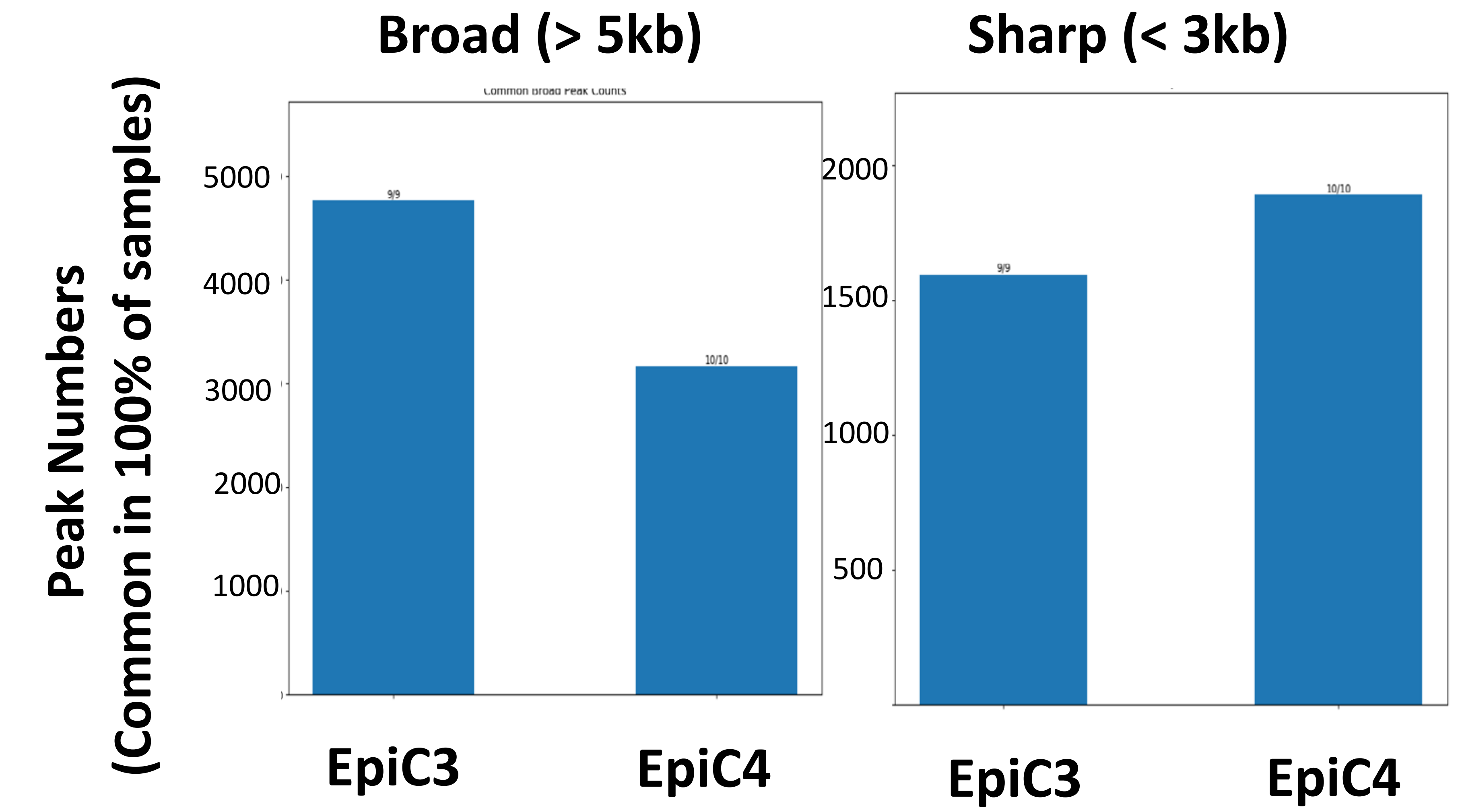



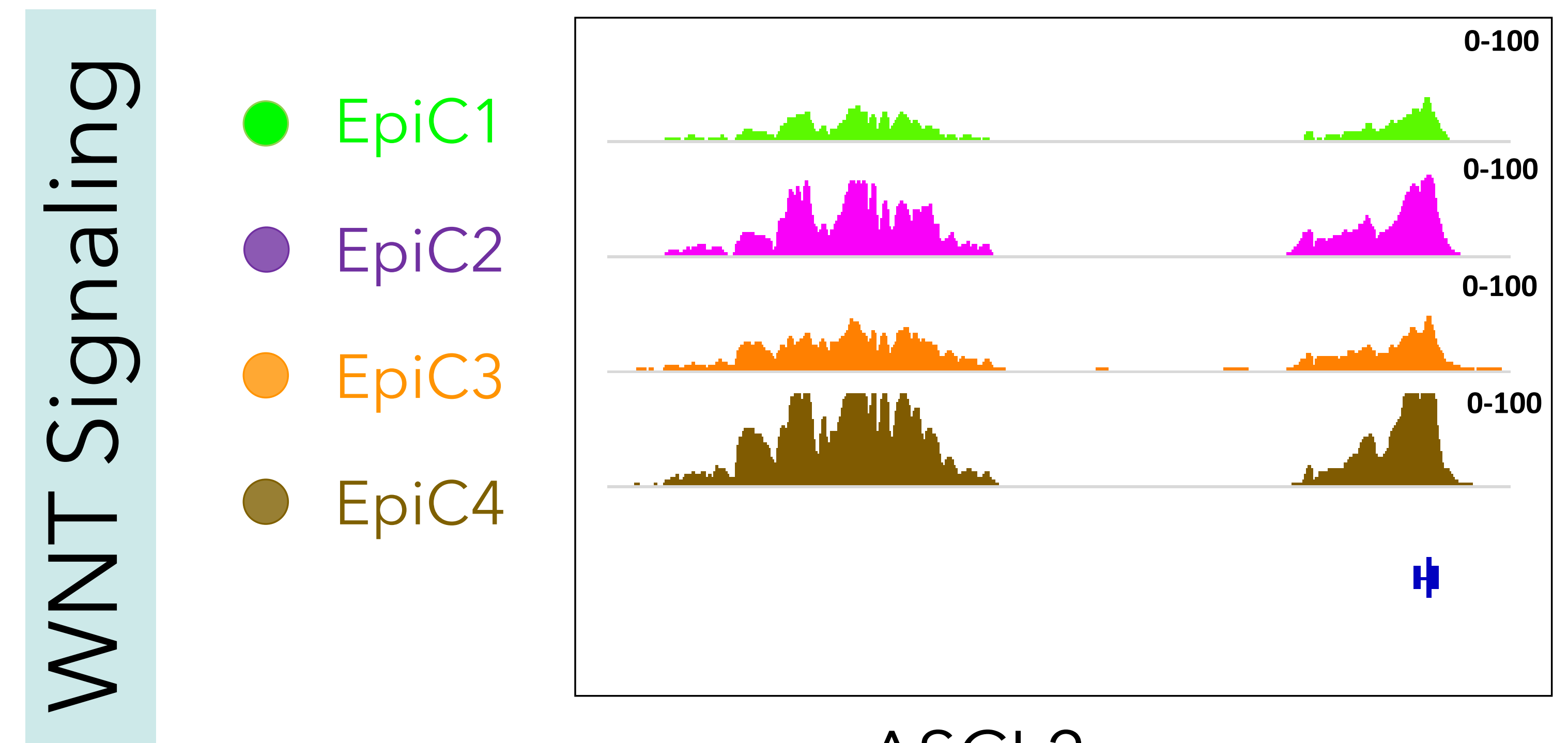

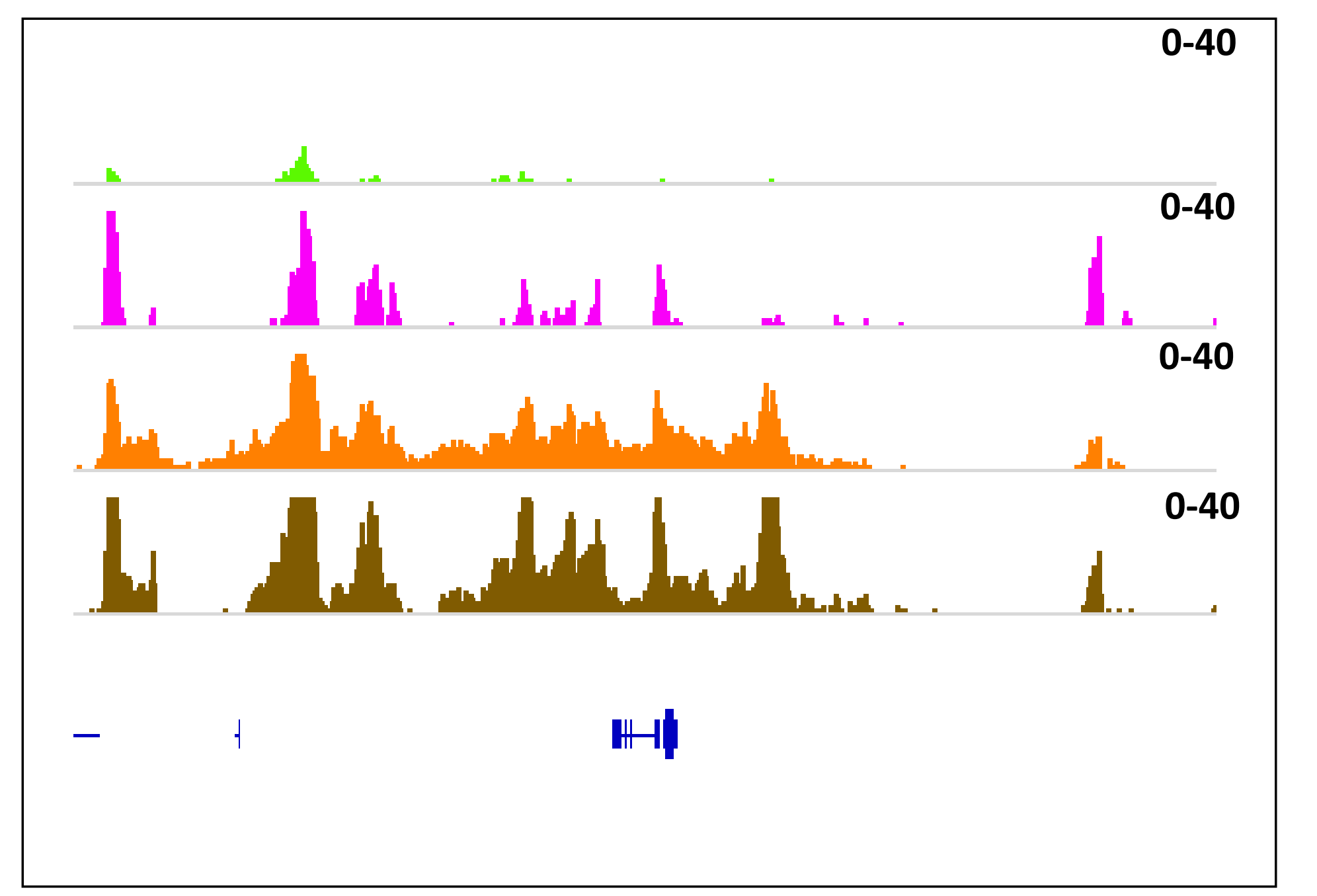

FZD10

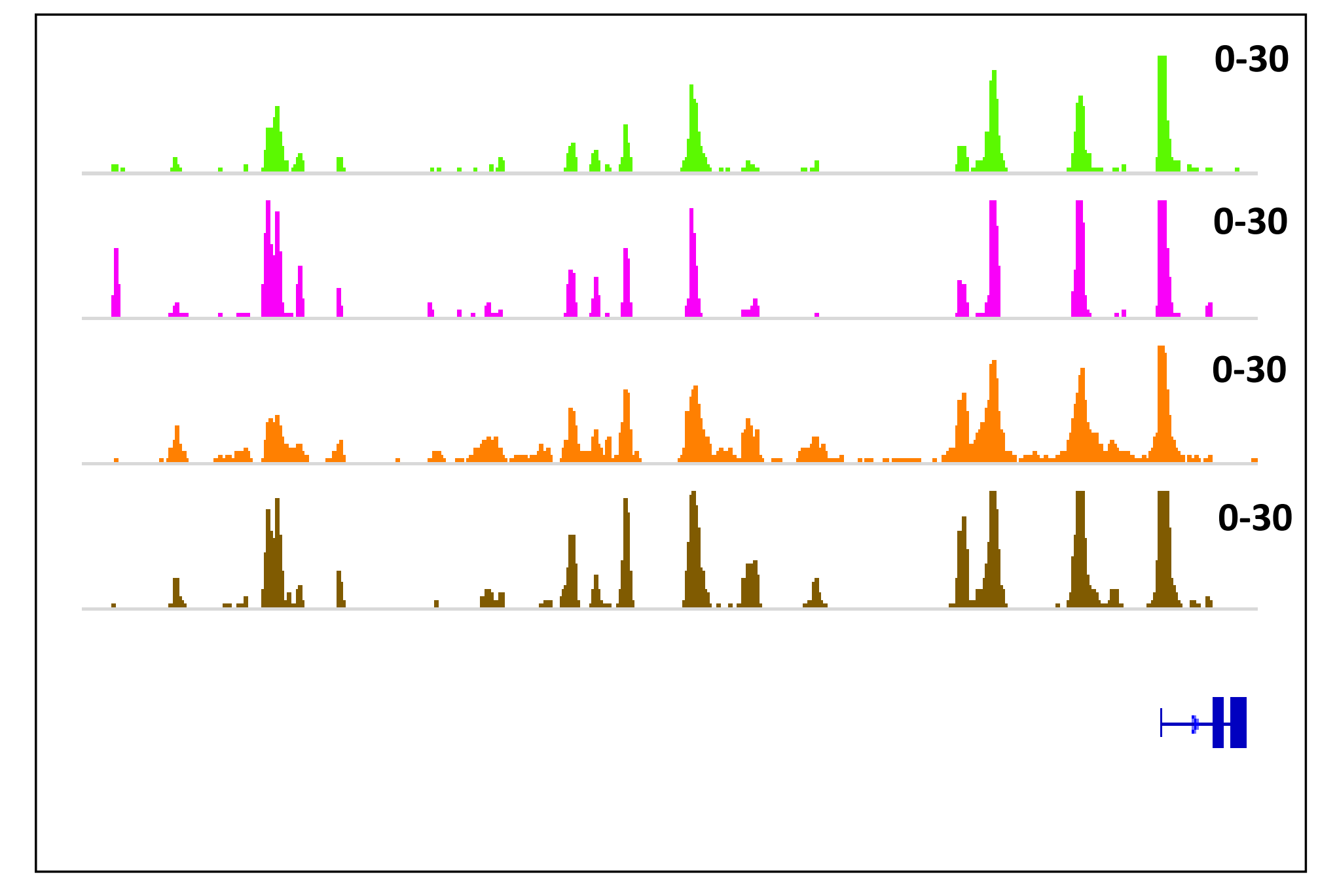

CTNN $\beta 1$
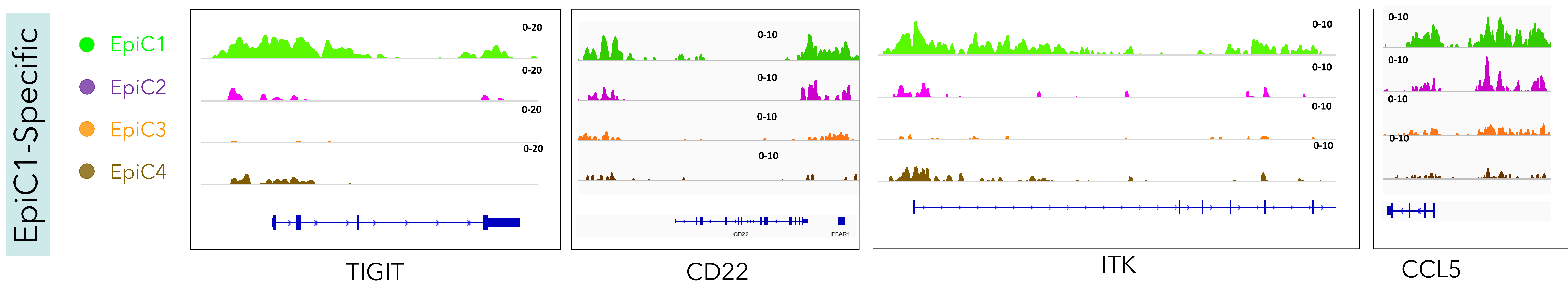

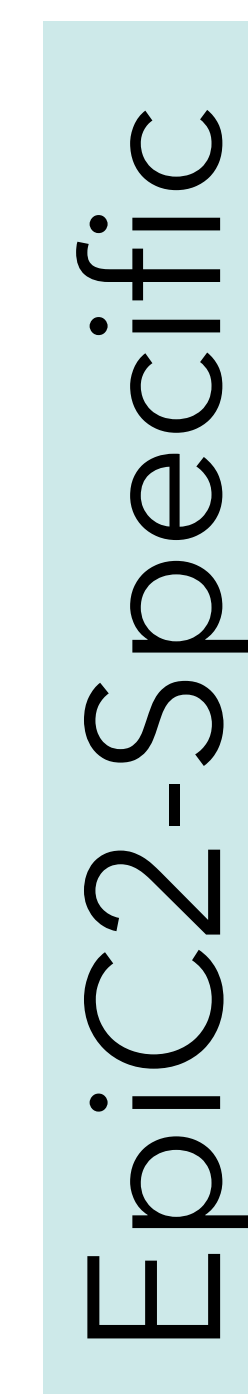

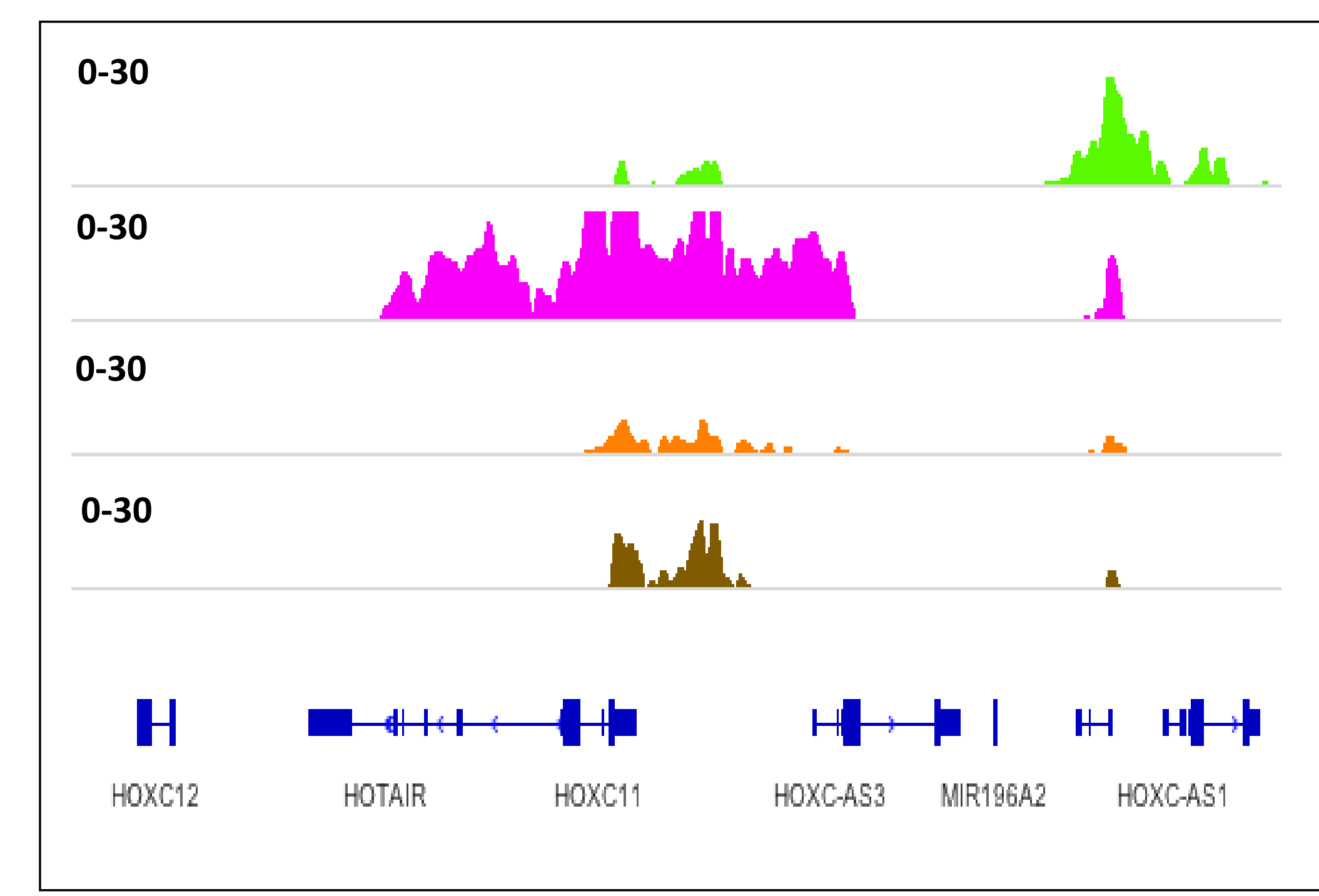

HOTAIR

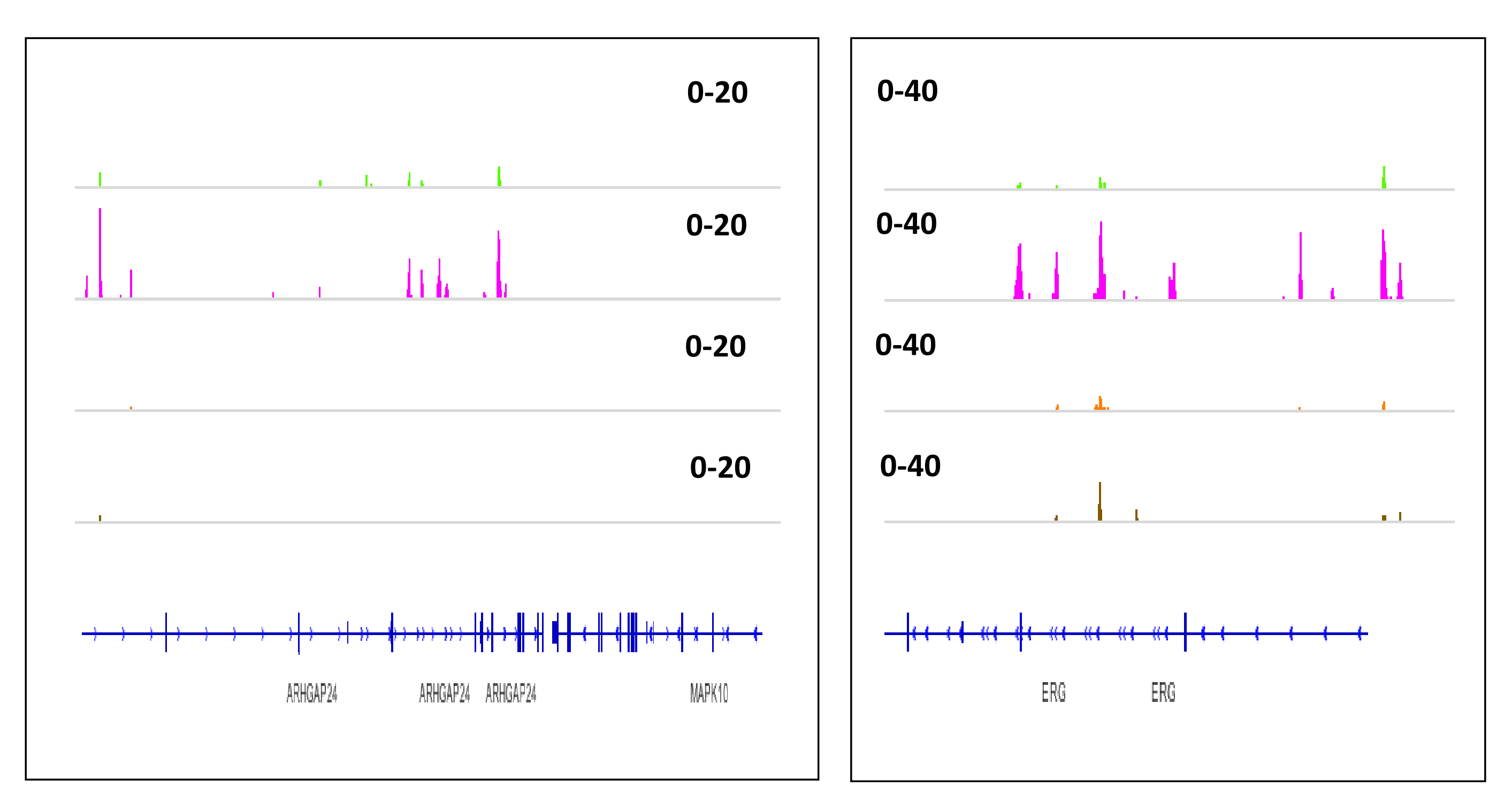

MAPK10

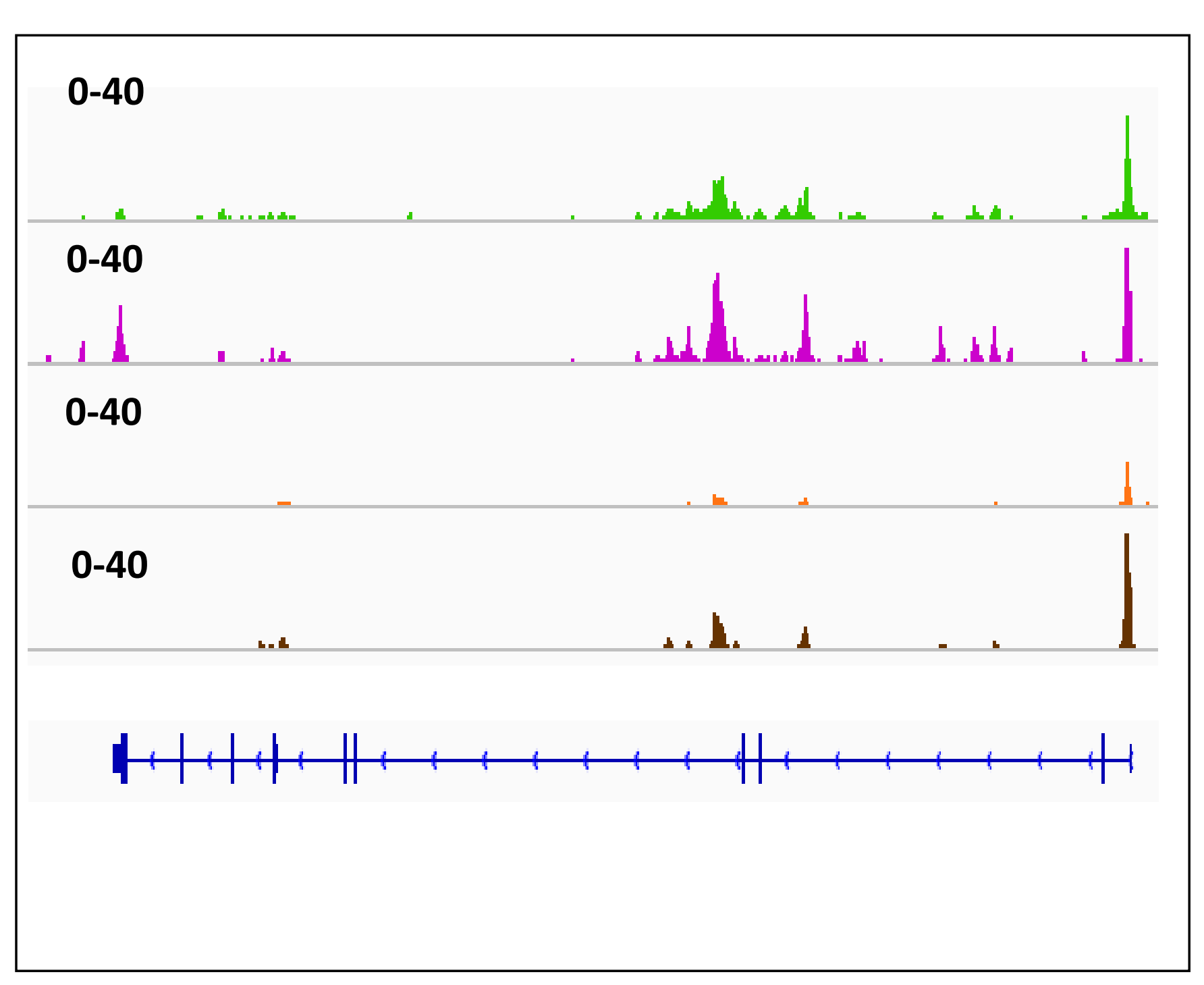

GLIS3
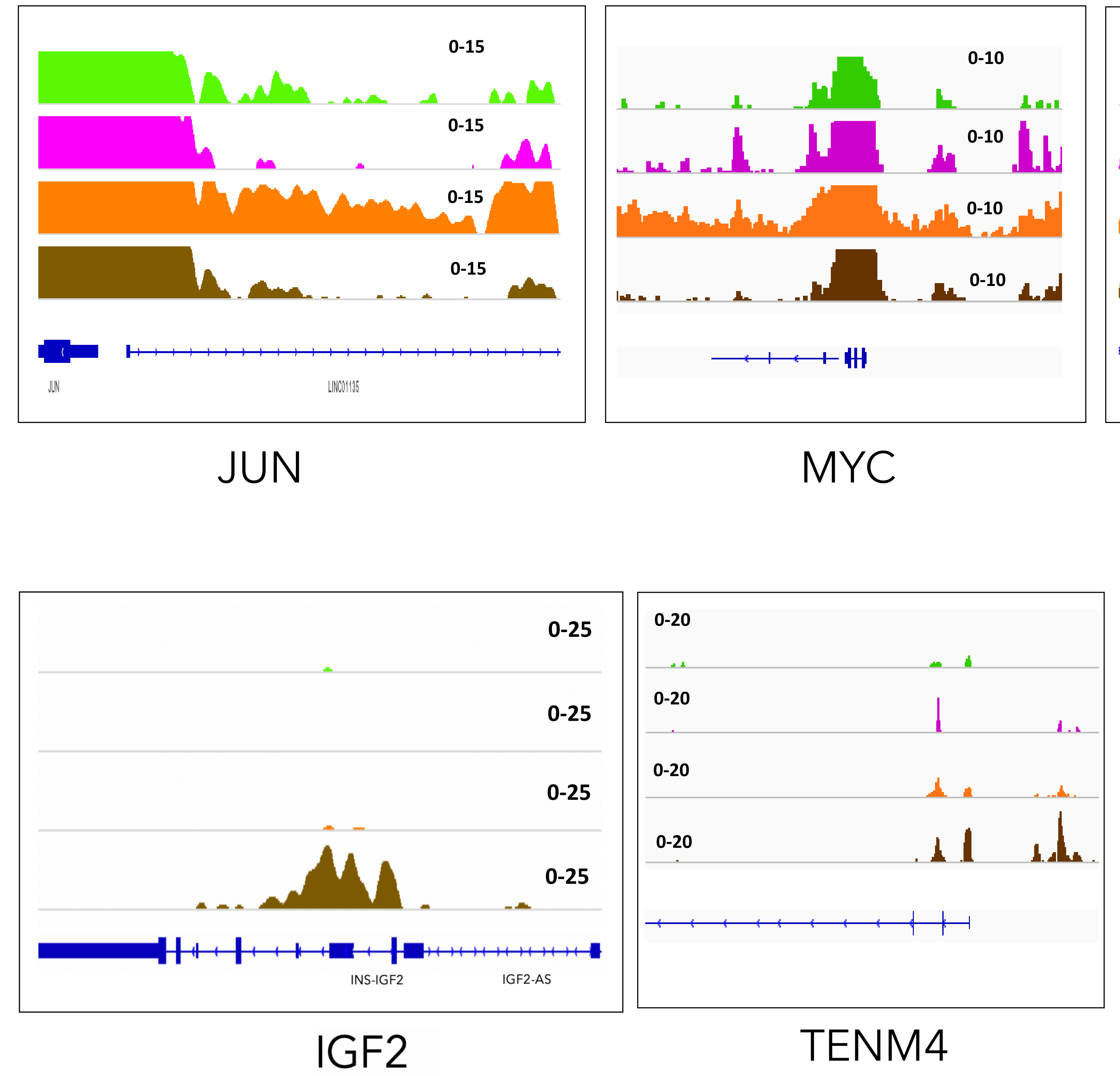

TENM4
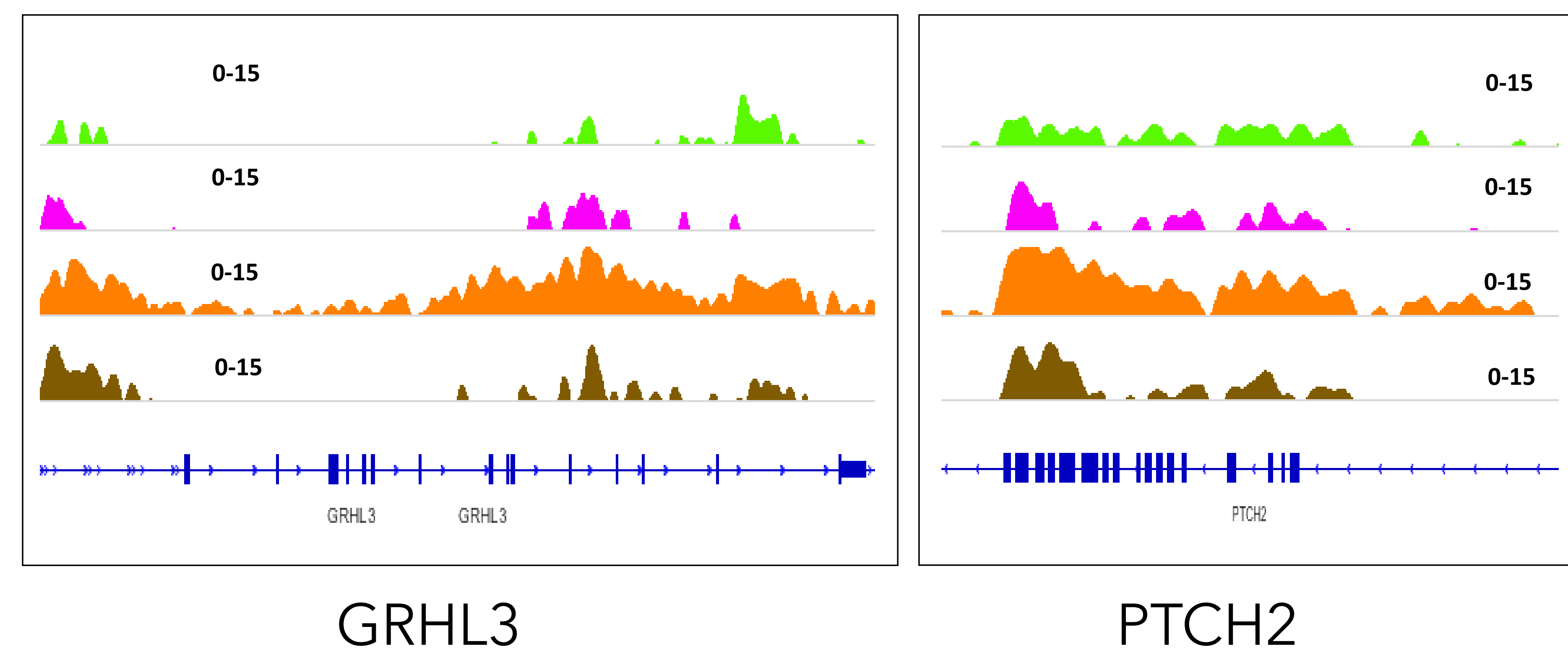

PTCH2
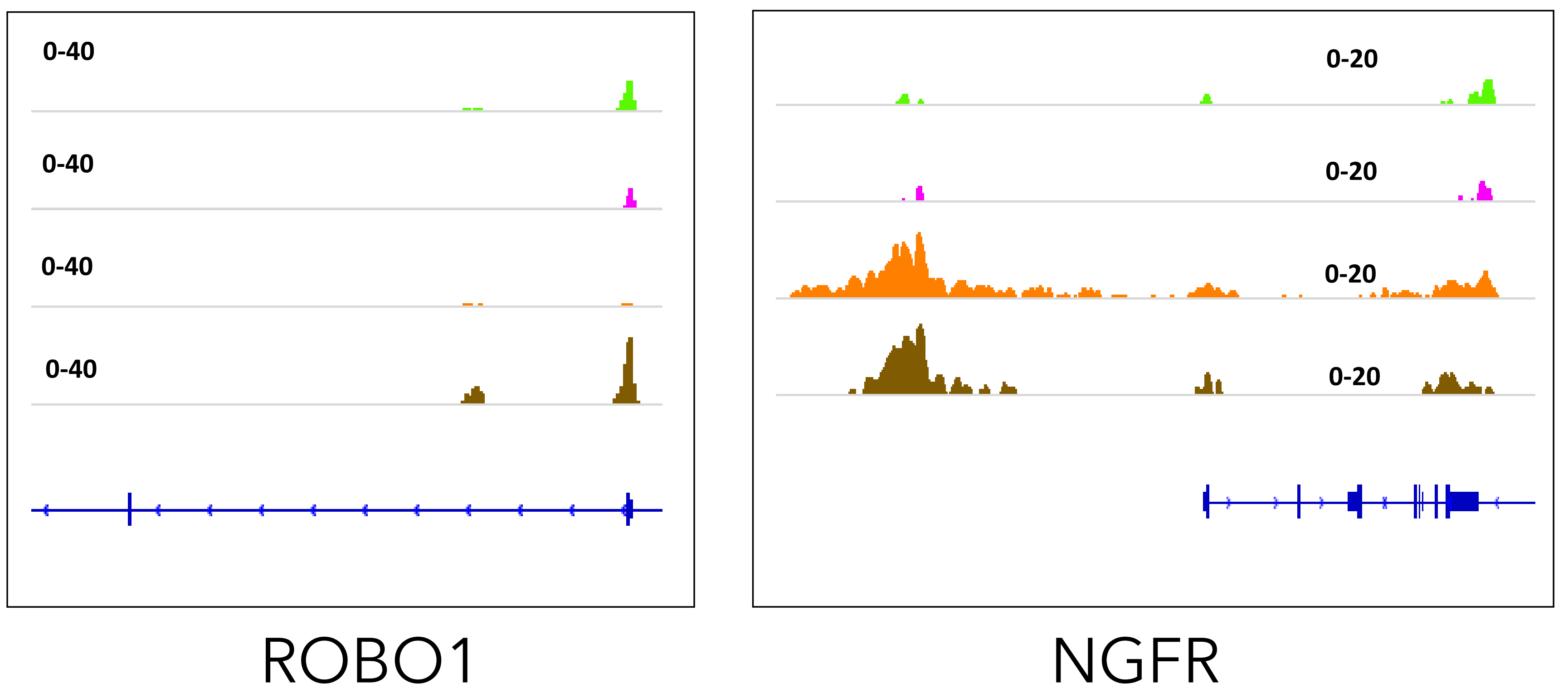

NGFR
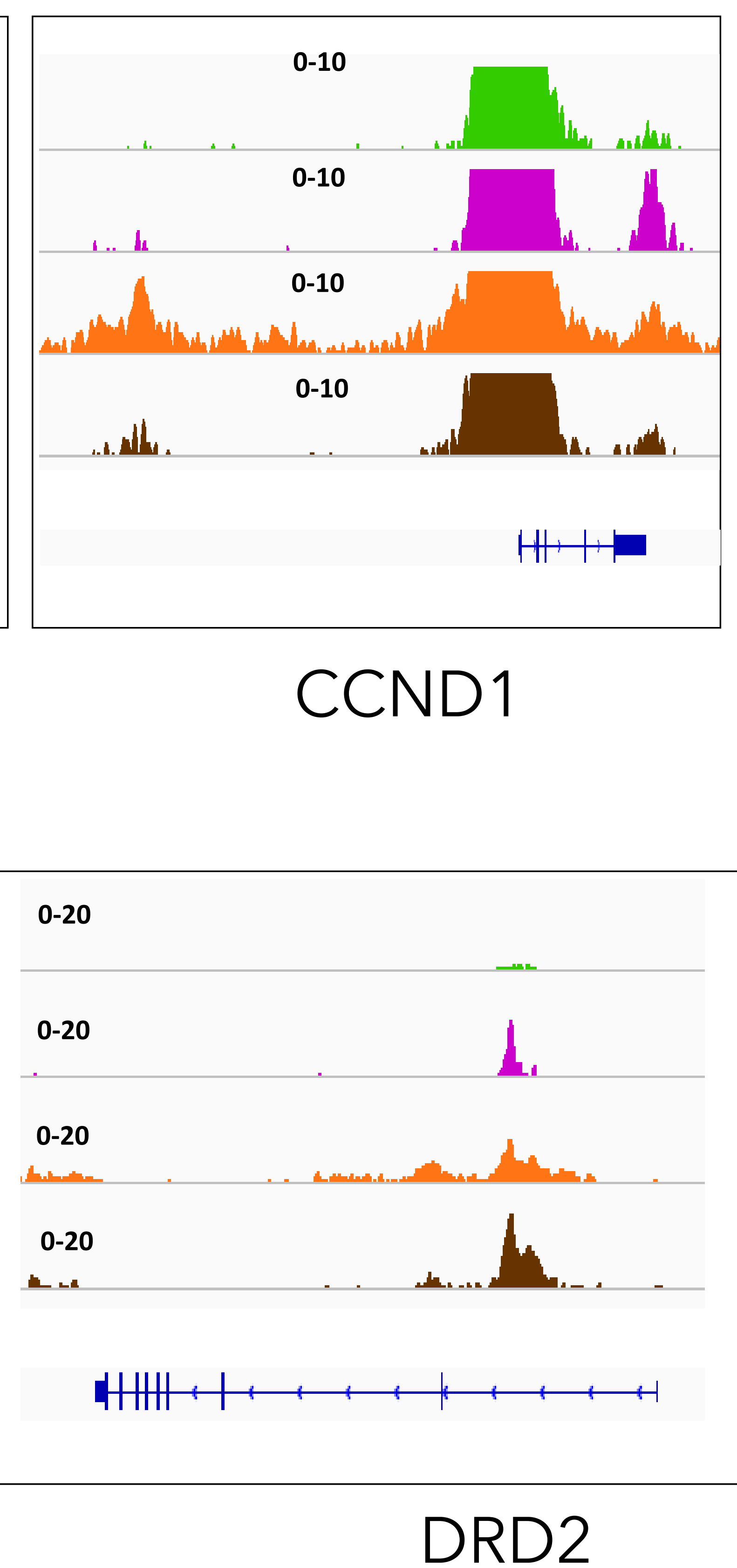


\section{Figure S9}

A

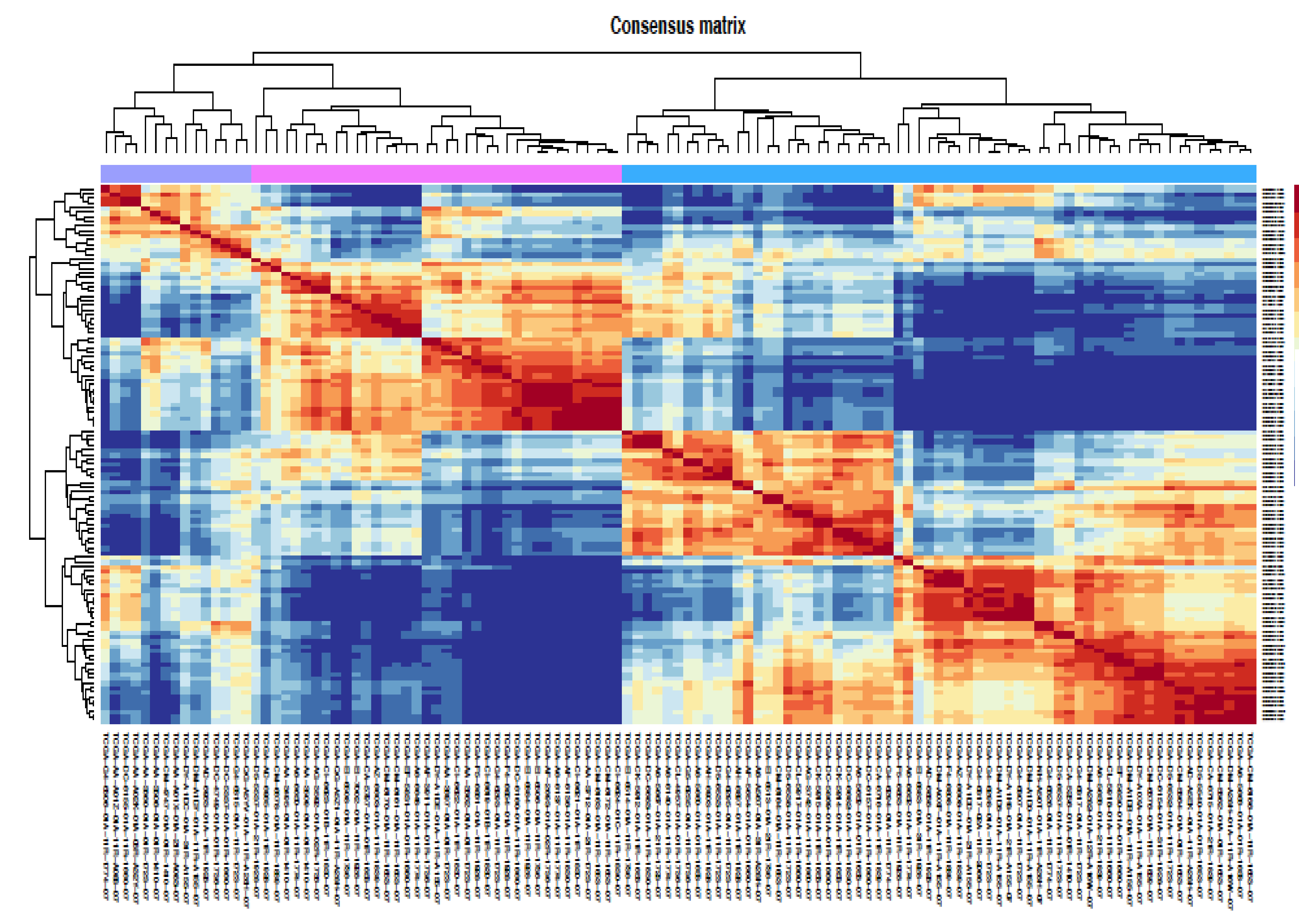

TCGA CMS2 Samples

C

EpiC3 Unique Genes overlap with Cluster 2 Genes

$\begin{aligned} \text { Pathway } & \text { FDR } \\ \text { Extracellular matrix organization } & 1.26 \mathrm{E}-04 \\ \text { Immune System } & 1.85 \mathrm{E}-04 \\ \text { GPCRs } & 3.01 \mathrm{E}-04 \\ \text { Renin-angiotensin system } & 4.35 \mathrm{E}-04 \\ \text { Keratan sulfate biosynthesis } & 7.87 \mathrm{E}-04\end{aligned}$

Cluster 1 vs Cluster 2 Survival

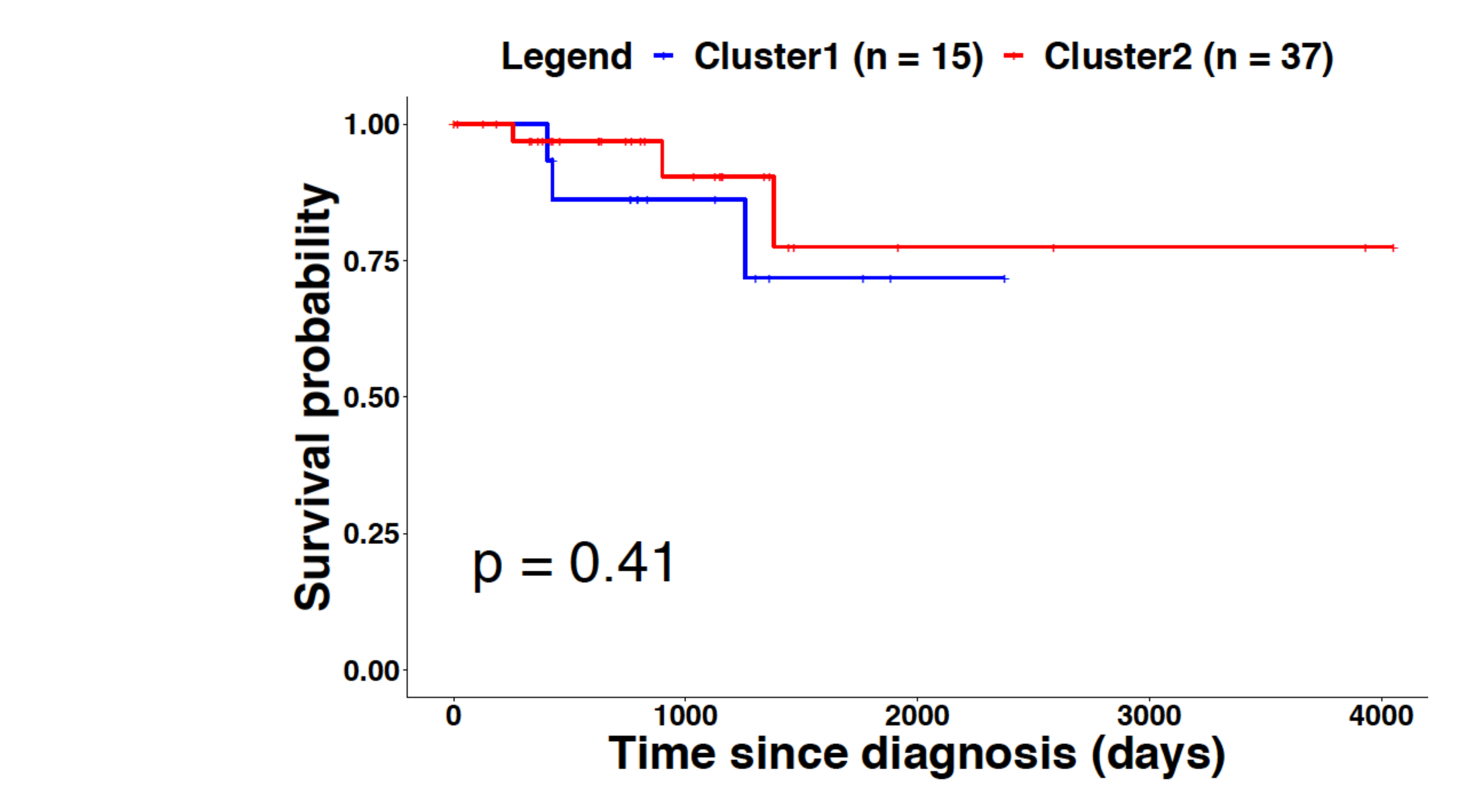

E

$\mathbf{F}$

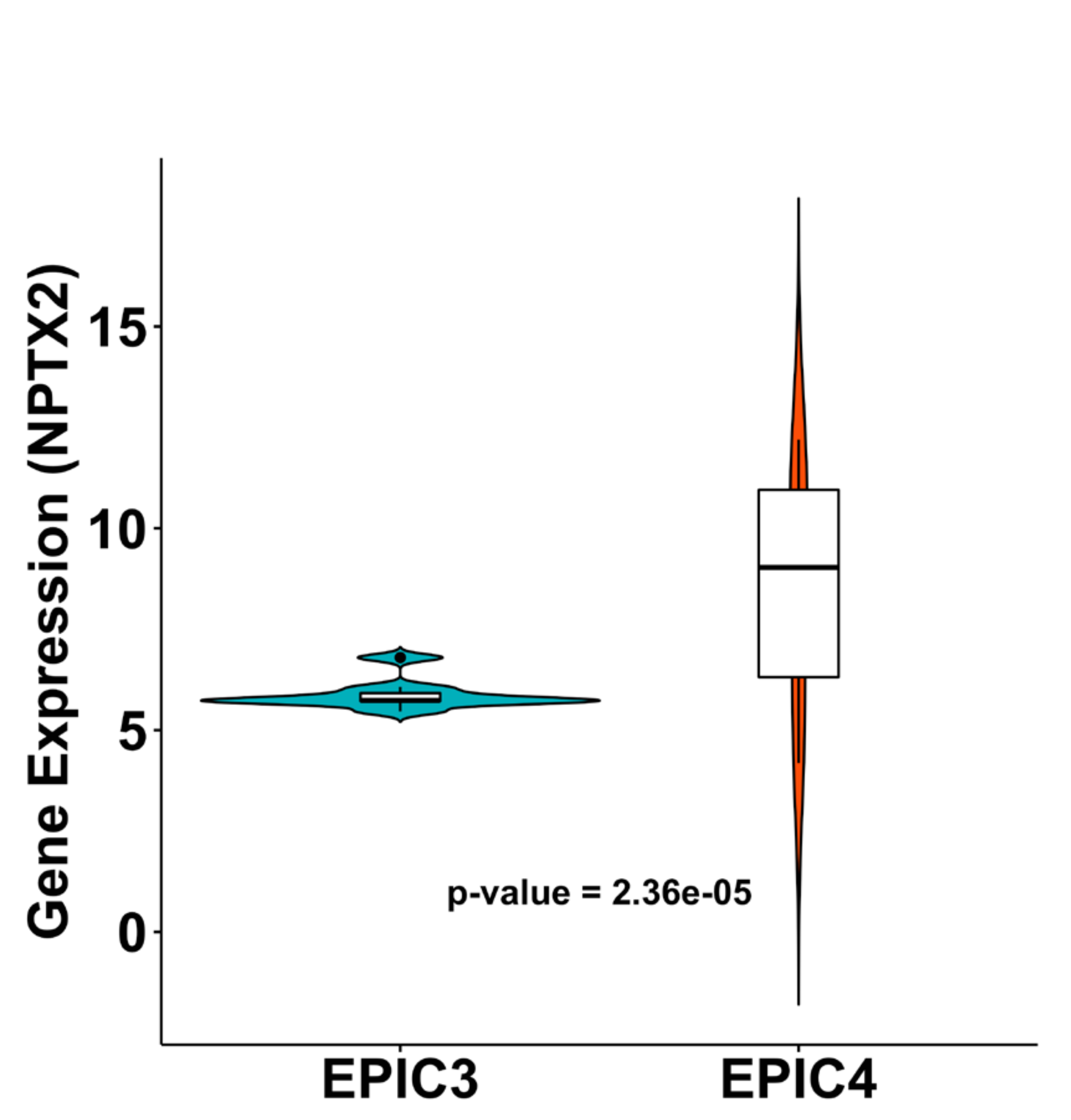

D
B

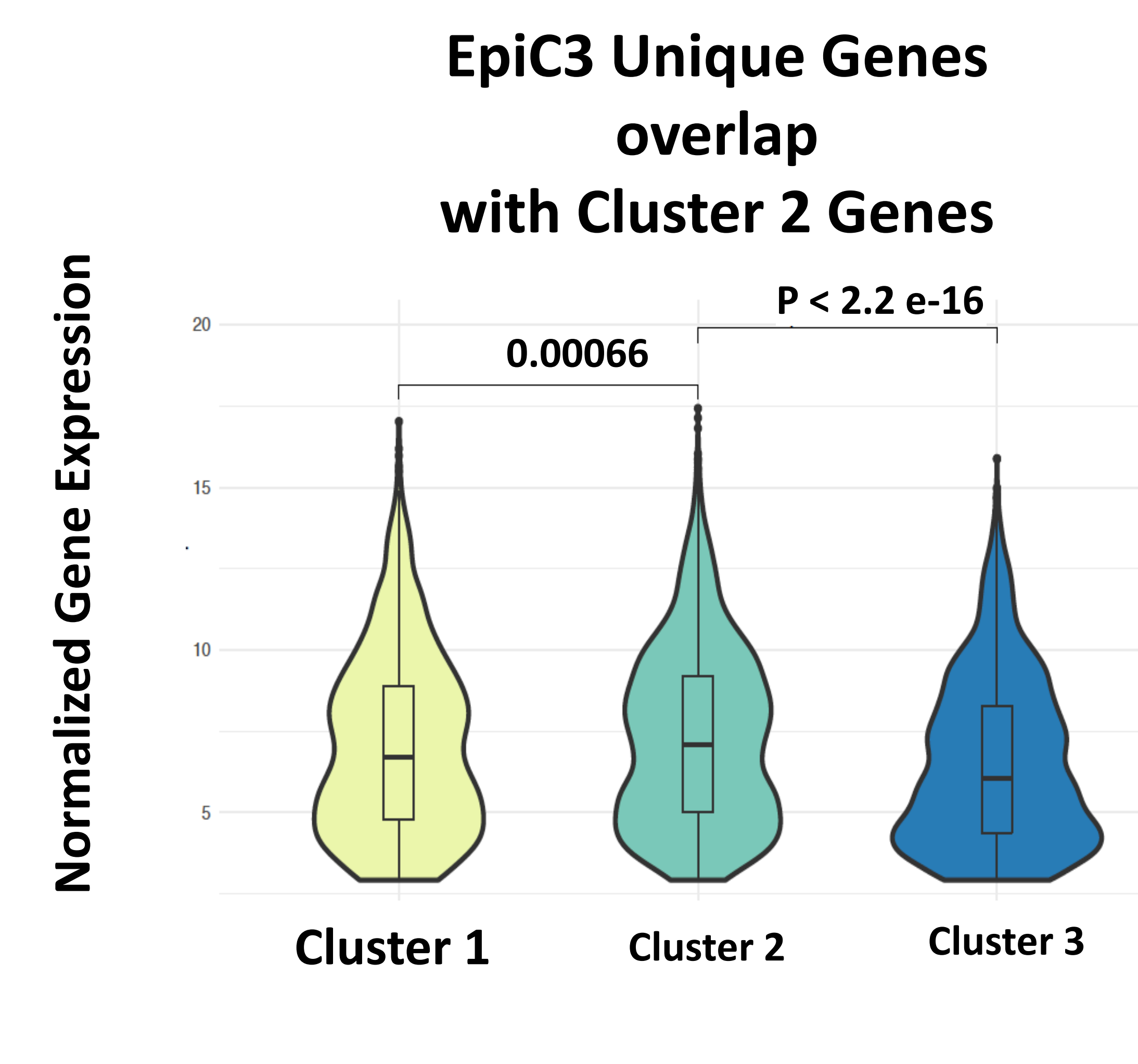
EpiC4 Unique Genes
overlap
with Cluster 2 Genes

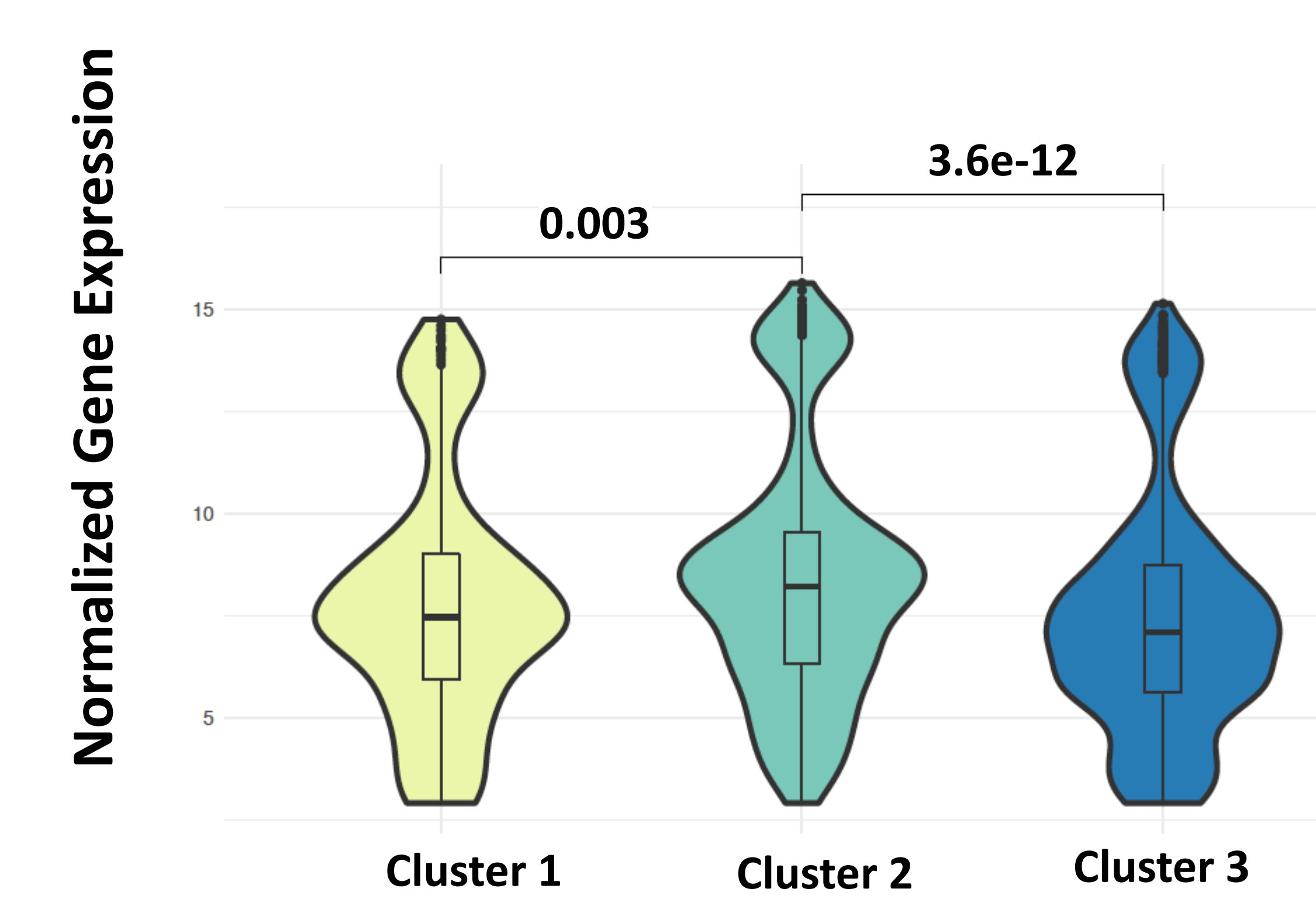

EpiC4 Unique Genes overlap with Cluster 3 Genes

\begin{tabular}{cc} 
Pathway & FDR \\
\hline Rap1 signaling pathway & $6.58 \mathrm{E}-03$ \\
Clsuter1 Specific genes &
\end{tabular}

\begin{tabular}{cr} 
Pathway & \multicolumn{1}{c}{ FDR } \\
Antigen processing & $2.34 \mathrm{E}-05$ \\
Class I MHC mediated antigen processing \& presentation & $7.02 \mathrm{E}-05$ \\
Ubiquitin mediated proteolysis & $9.56 \mathrm{E}-05$ \\
RIG-I-like Receptor Signaling & 0.0005179 \\
il-2 receptor beta chain in t cell activation & 0.000666397
\end{tabular}

Cluster 1 vs Cluster 3 Survival

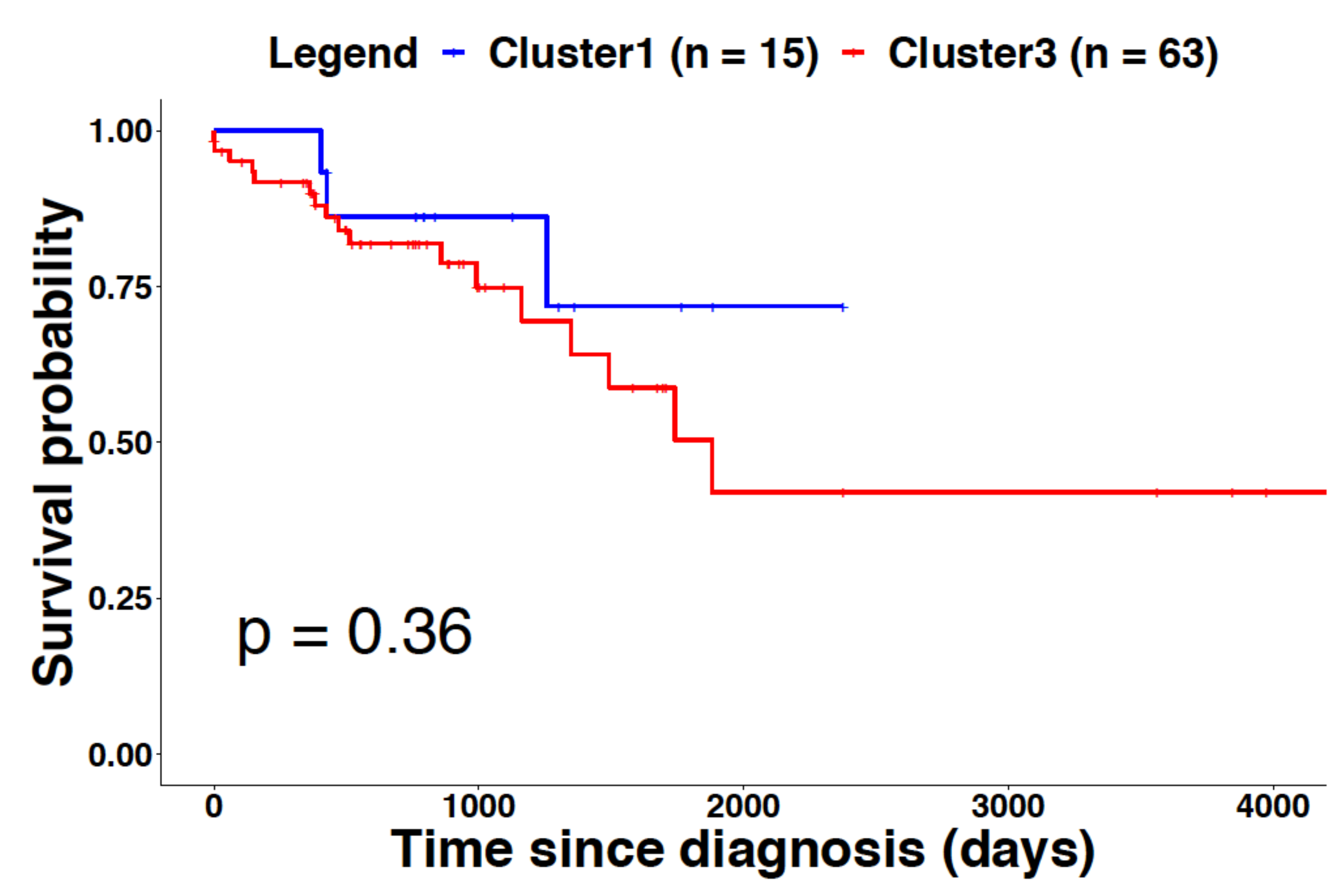

EpiC3 Unique Genes

overlap

with Cluster 3 Genes

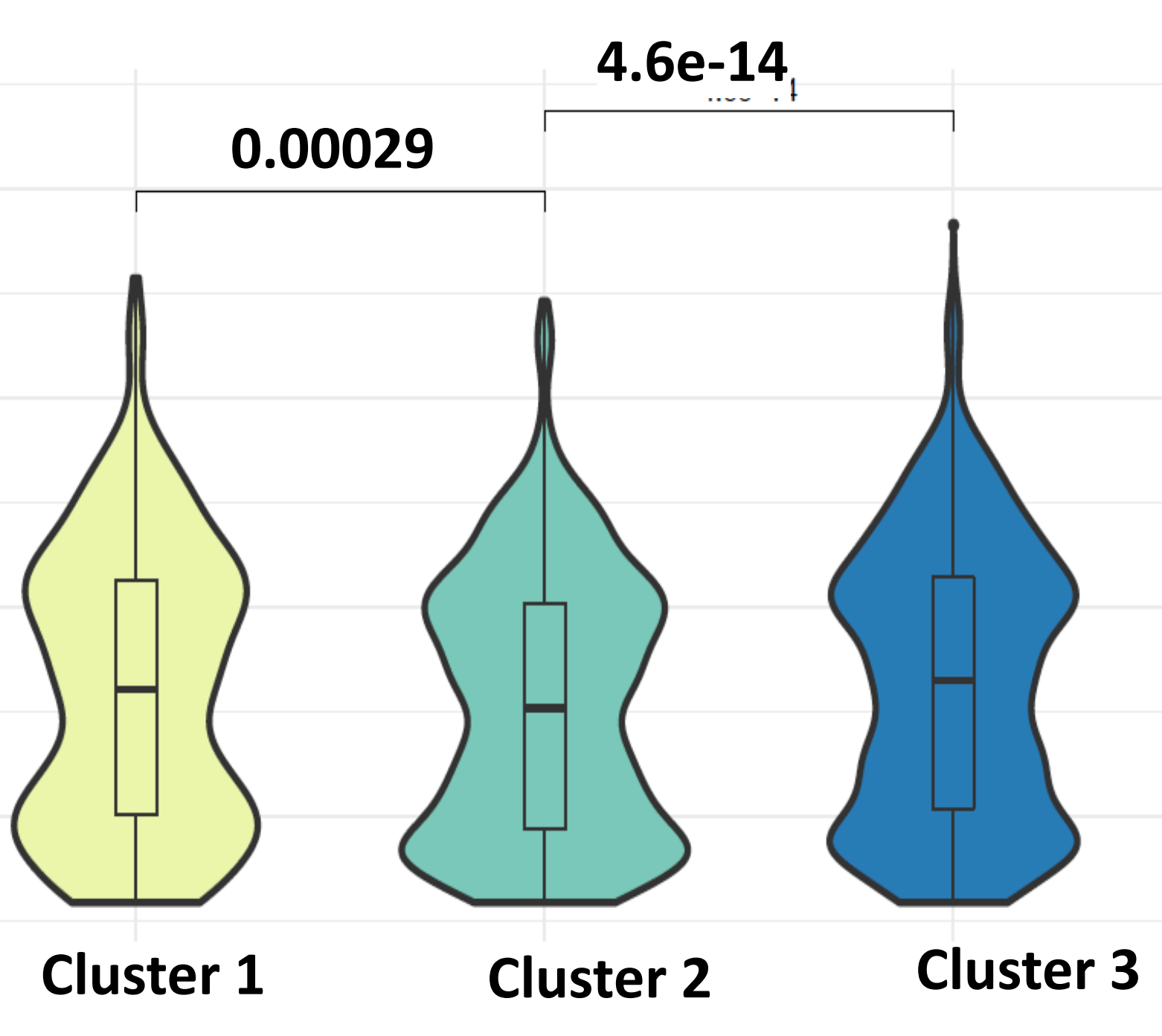

EpiC4 Unique Genes

overlap

with Cluster 3 Genes

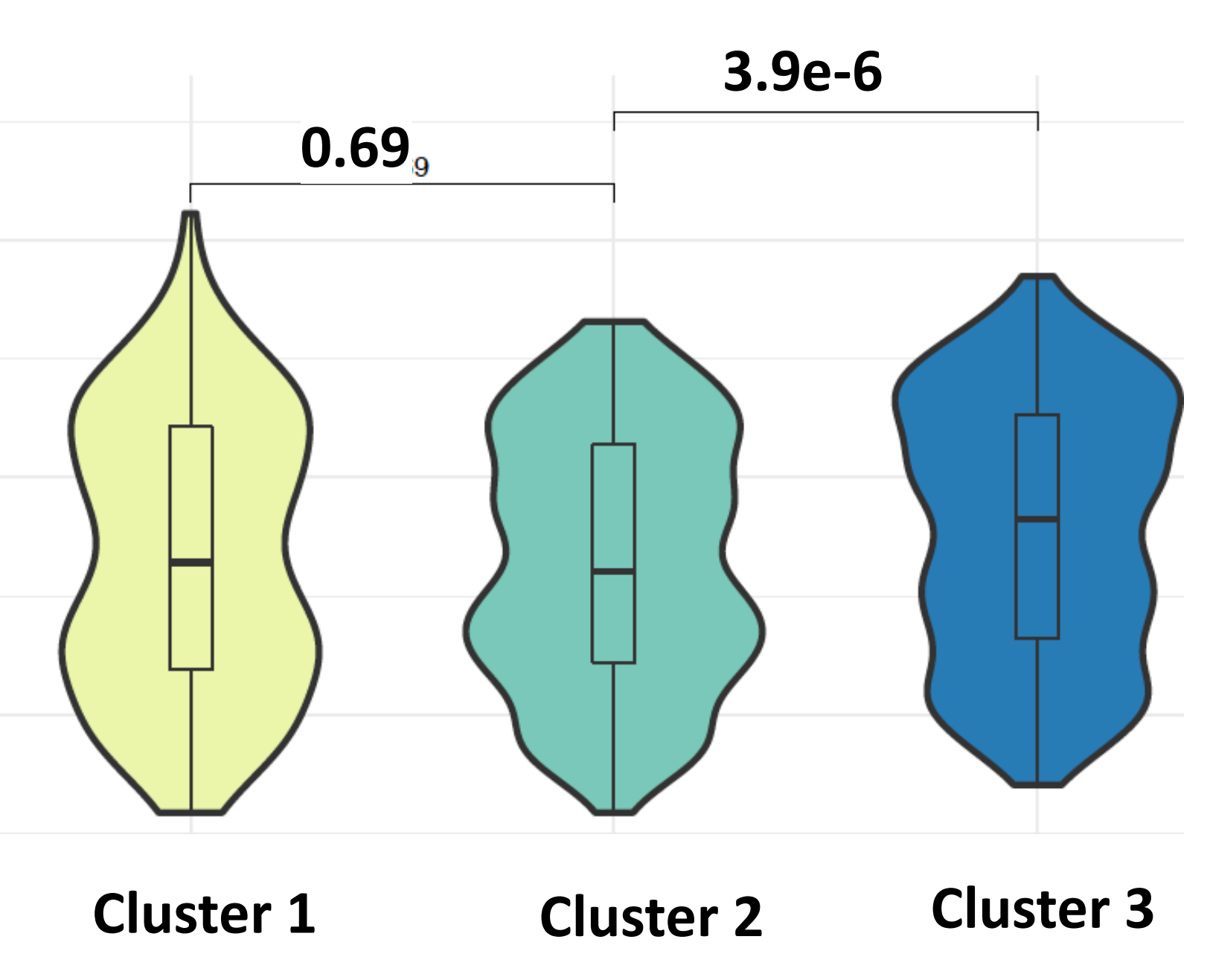

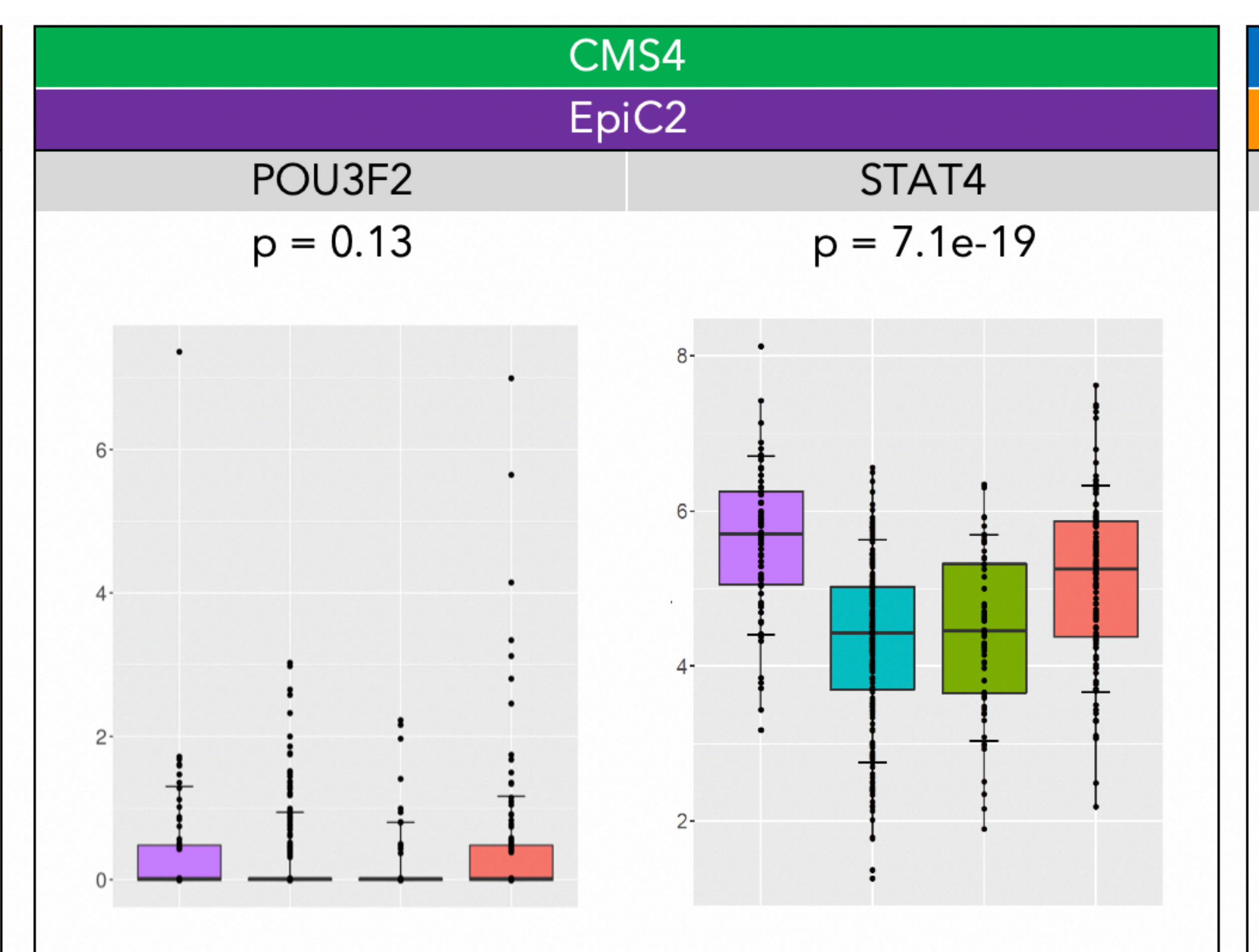

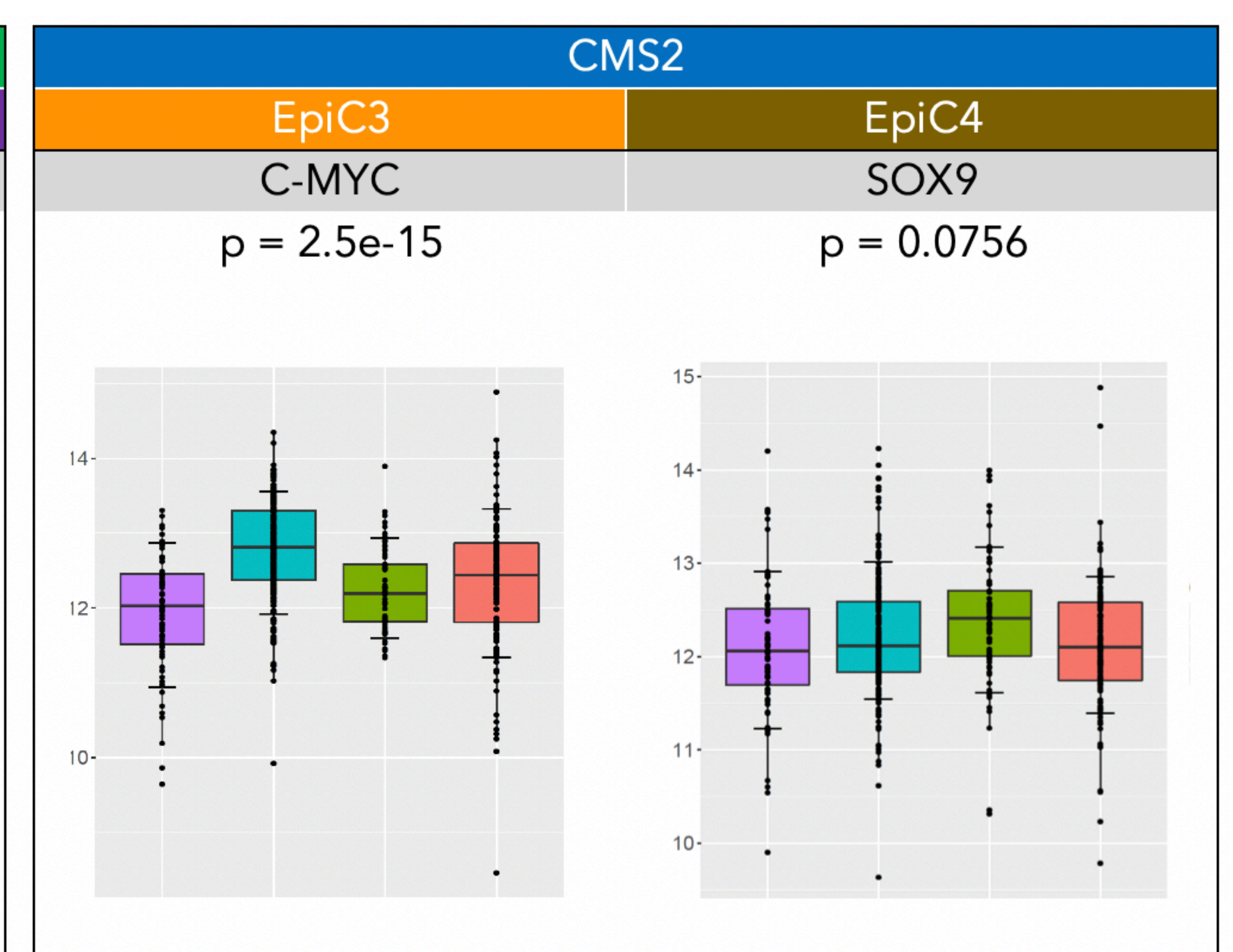


A

Bromodomain and CBP Inhibitors

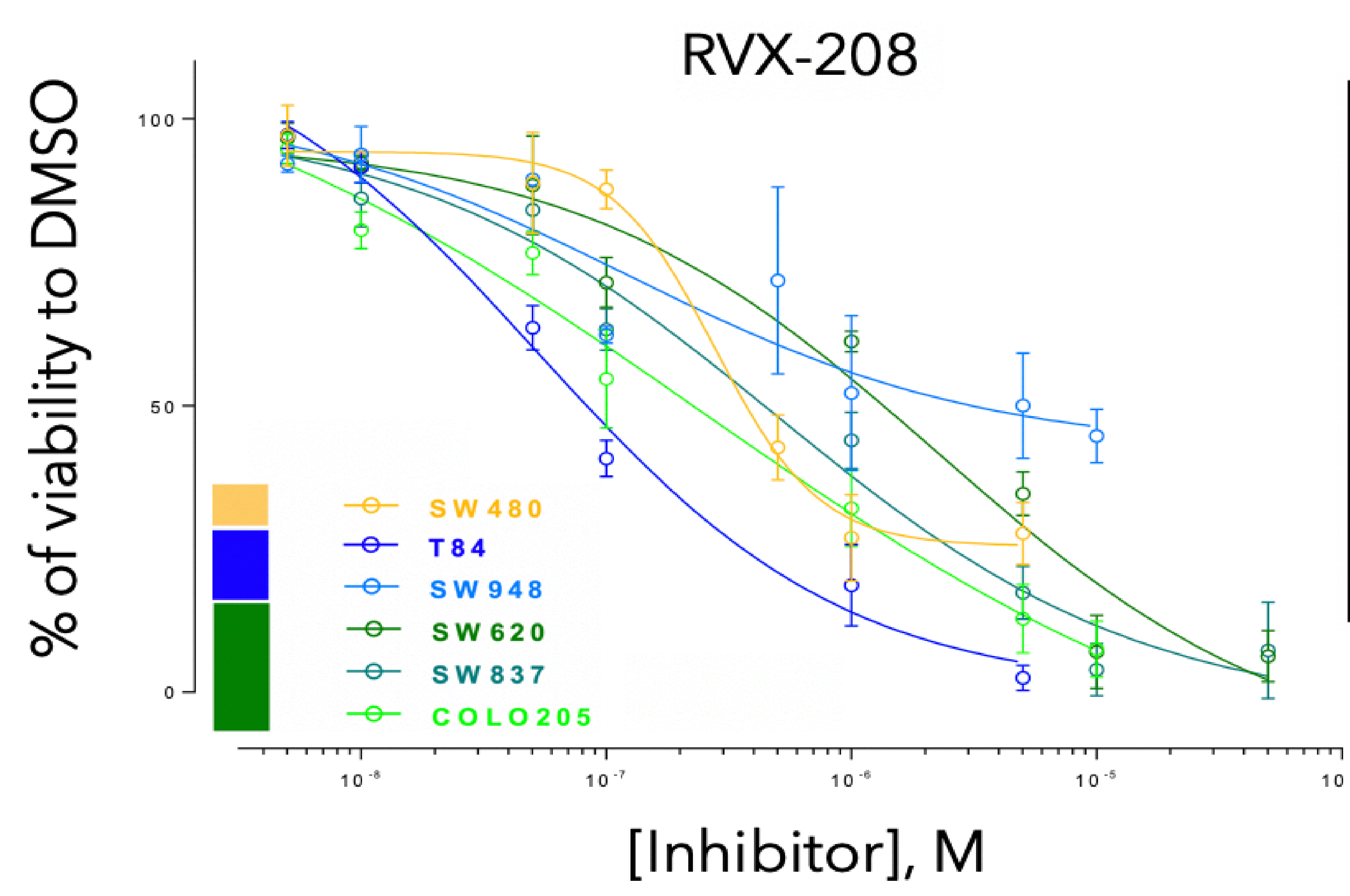

\begin{tabular}{|c|c|}
\hline & RVX208 \\
\hline SW480 & $1.61 \mathrm{E}-04$ \\
\hline SW948 & $5.07 \mathrm{E}-04$ \\
\hline T84 & $7.53 \mathrm{E}-05$ \\
\hline SW620 & $6.30 \mathrm{E}-04$ \\
\hline SW837 & $4.55 \mathrm{E}-04$ \\
\hline COLO205 & $1.87 \mathrm{E}-04$ \\
\hline
\end{tabular}

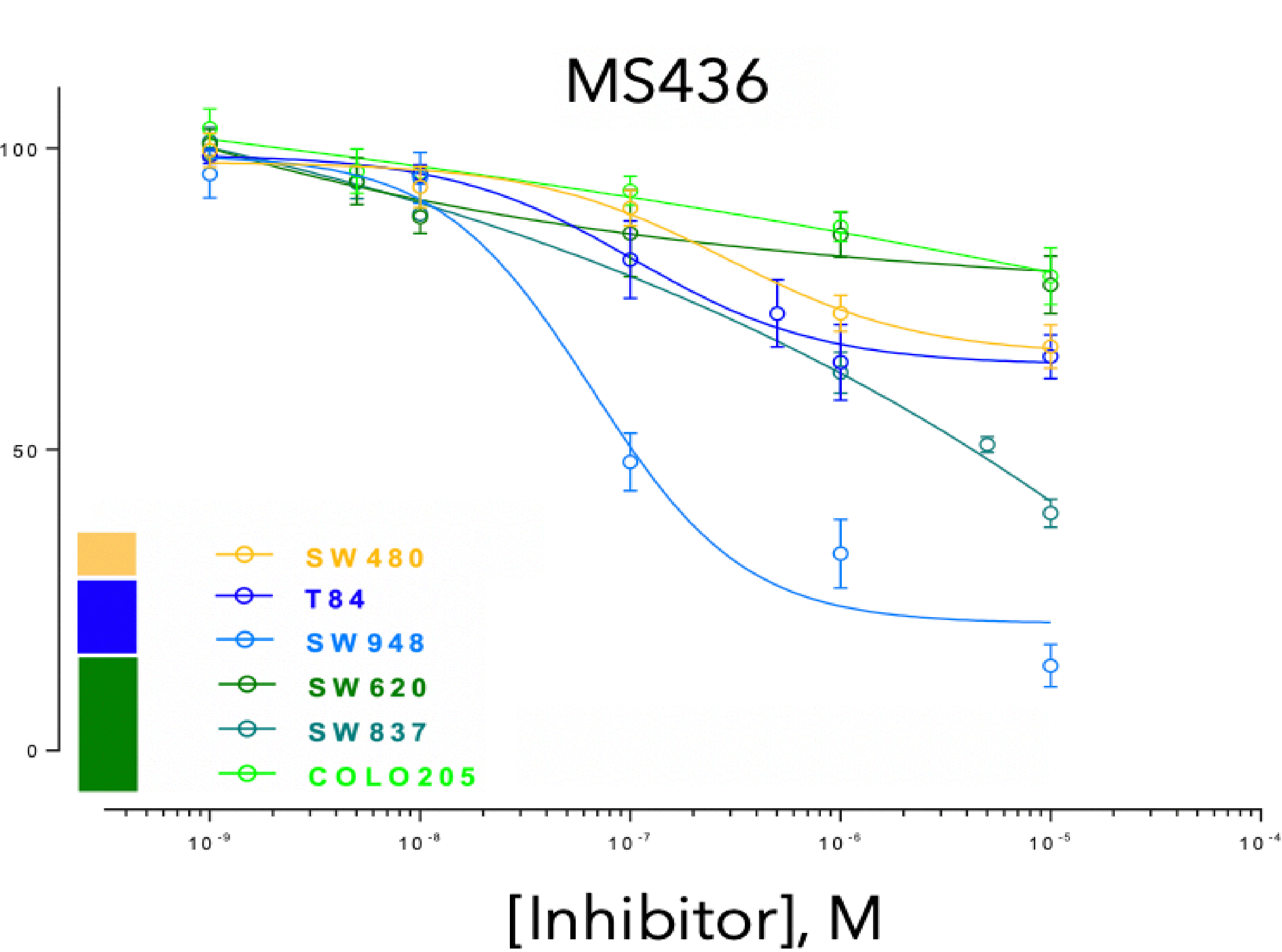

\begin{tabular}{|c|c|}
\hline & $M S 436$ \\
\hline SW480 & $7.11 \mathrm{E}-04$ \\
\hline SW948 & $2.54 \mathrm{E}-04$ \\
\hline T84 & $6.58 \mathrm{E}-04$ \\
\hline SW620 & $8.19 \mathrm{E}-04$ \\
\hline SW837 & $5.29 \mathrm{E}-04$ \\
\hline COLO205 & $8.36 \mathrm{E}-04$ \\
\hline
\end{tabular}

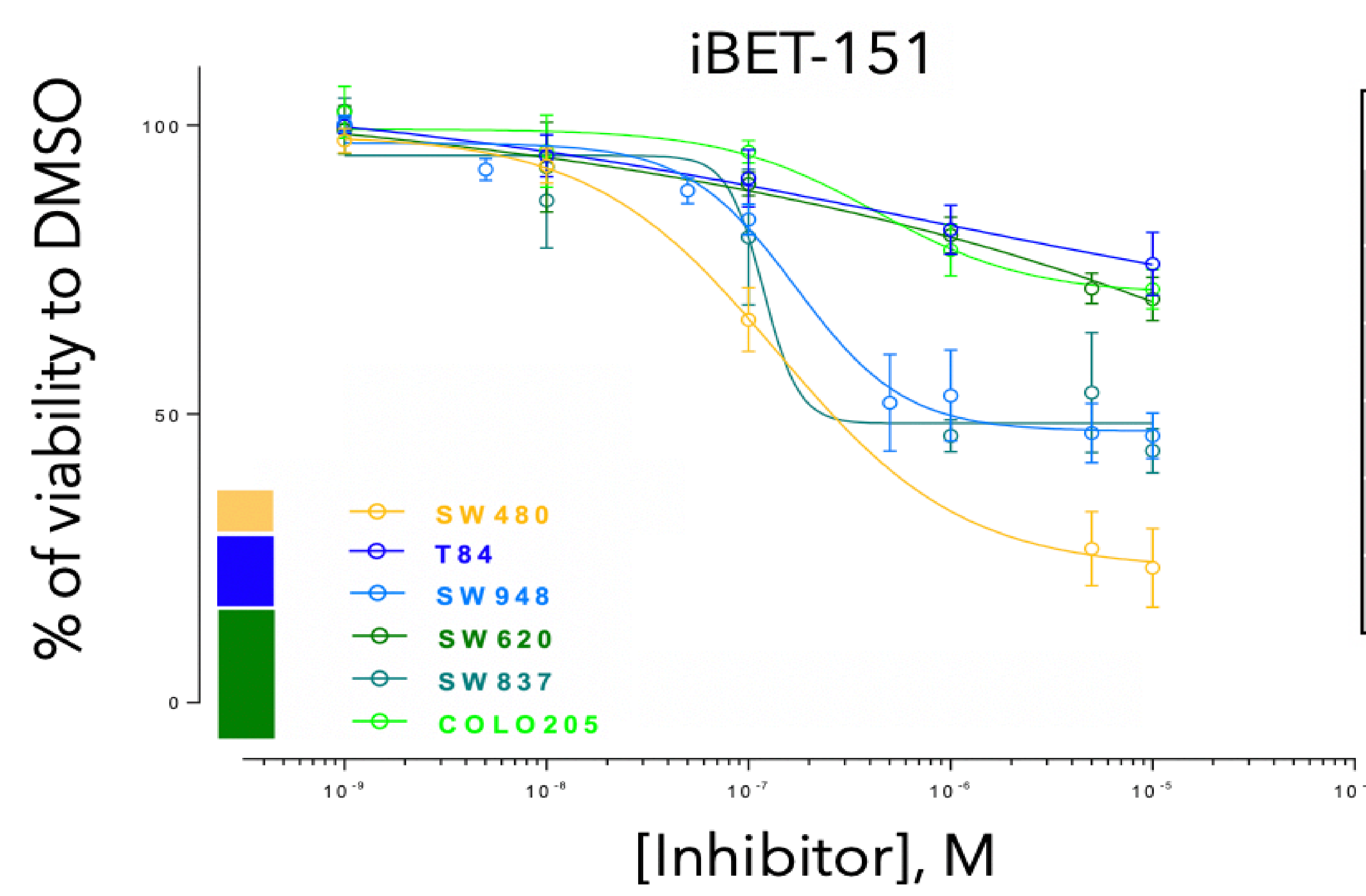

\begin{tabular}{|c|c|}
\hline & i-BET151 \\
\hline SW480 & $3.61 \mathrm{E}-04$ \\
SW948 & $4.94 \mathrm{E}-04$ \\
\hline T84 & $7.98 \mathrm{E}-04$ \\
SW620 & $7.46 \mathrm{E}-04$ \\
\hline SW837 & $5.09 \mathrm{E}-04$ \\
COLO205 & $7.63 \mathrm{E}-04$ \\
\hline
\end{tabular}

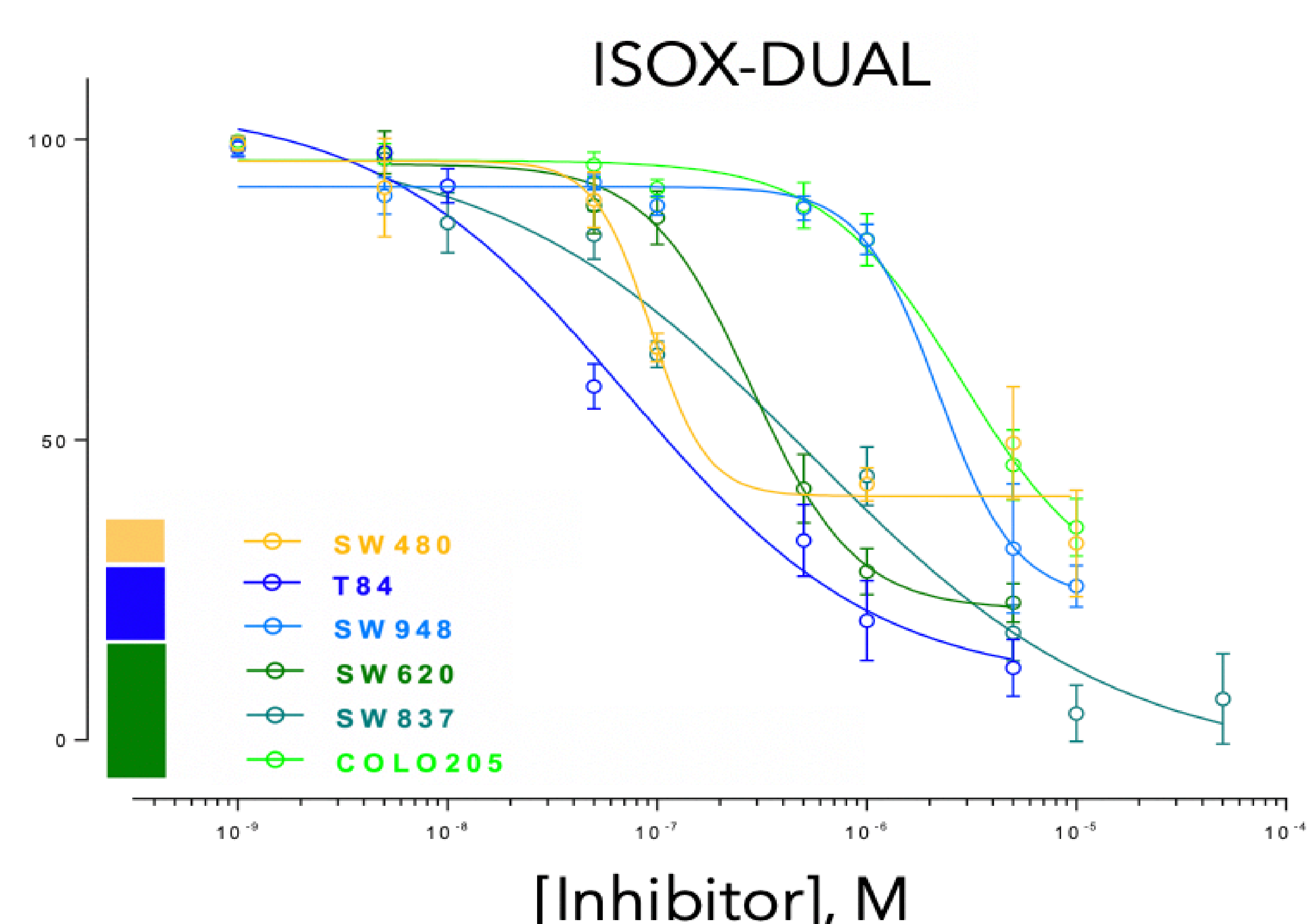

\begin{tabular}{|c|c|}
\hline & ISOX DUAL \\
\hline SW480 & $4.47 \mathrm{E}-04$ \\
\hline SW948 & $4.62 \mathrm{E}-04$ \\
\hline T84 & $1.02 \mathrm{E}-04$ \\
SW620 & $1.54 \mathrm{E}-04$ \\
\hline SW837 & $4.62 \mathrm{E}-04$ \\
\hline COLO205 & $5.50 \mathrm{E}-04$ \\
\hline
\end{tabular}
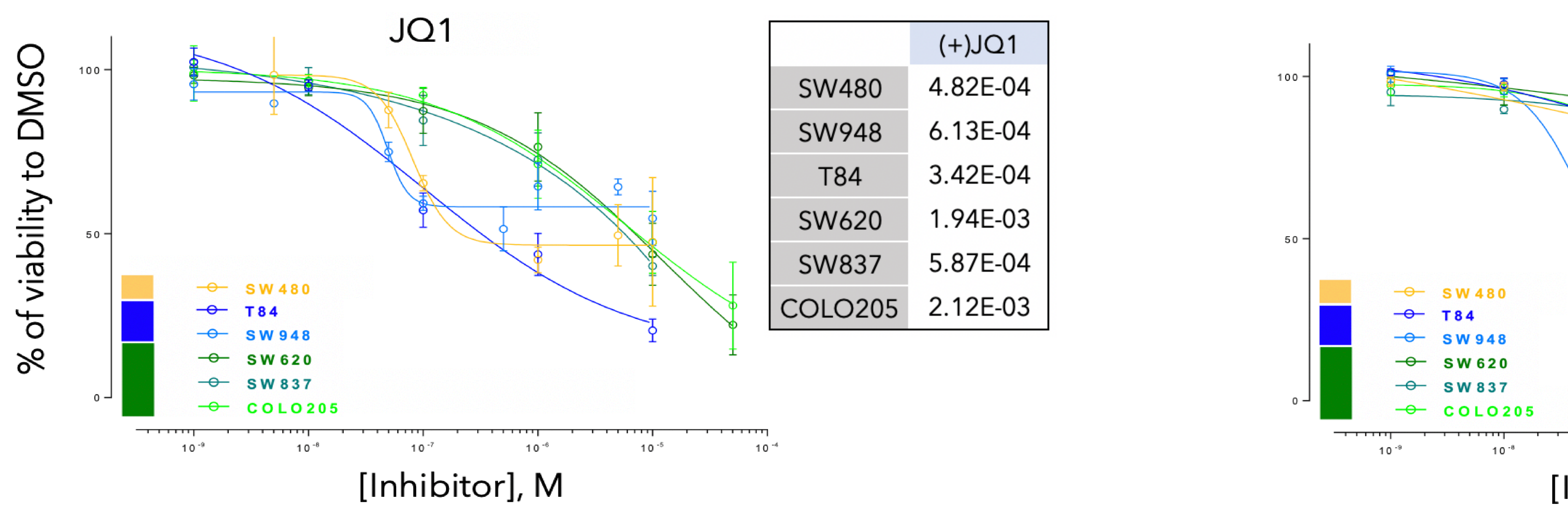

BI9564

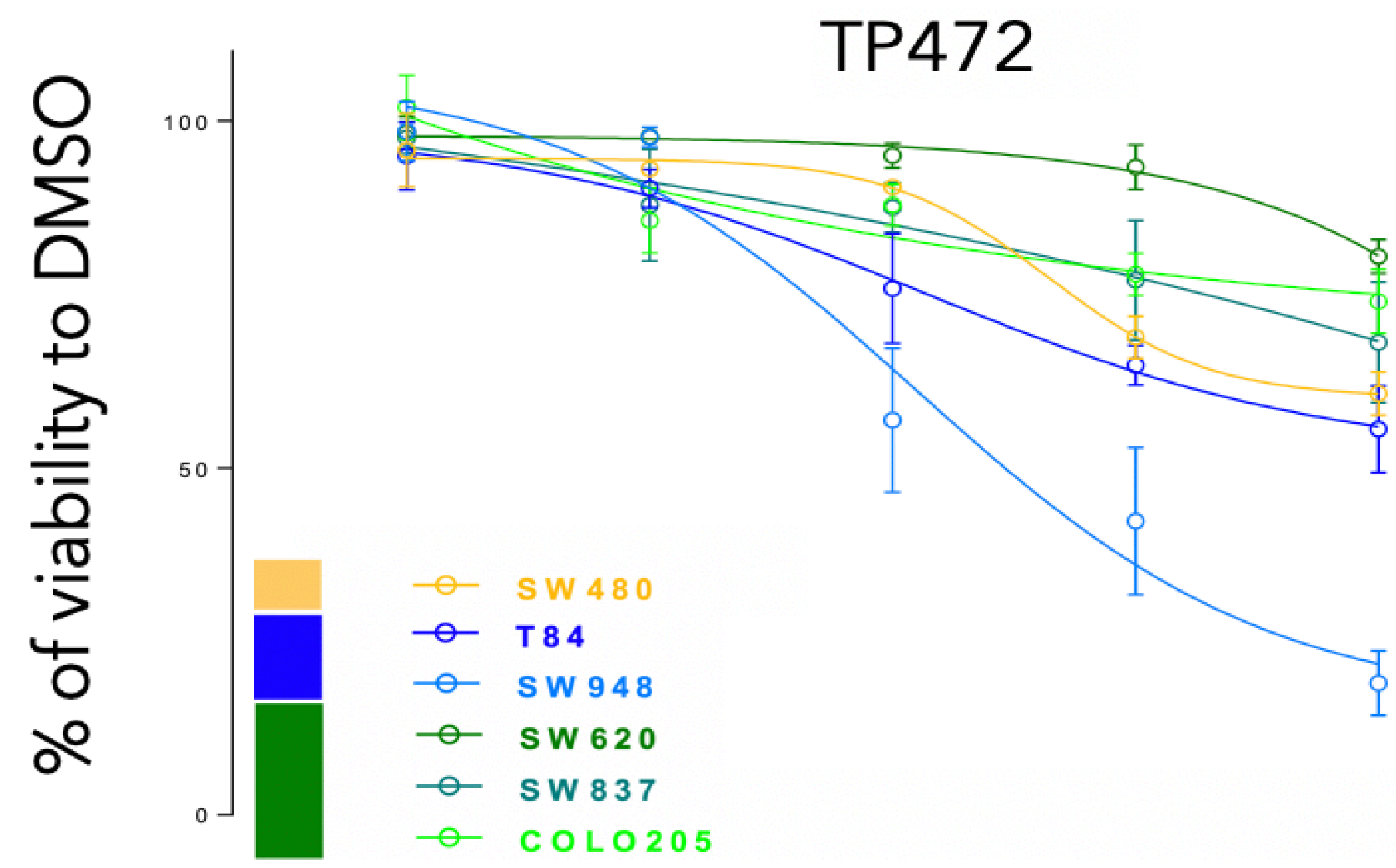

\begin{tabular}{|c|c|}
\hline & TP472 \\
\hline SW480 & $6.64 \mathrm{E}-04$ \\
\hline SW948 & $3.29 \mathrm{E}-04$ \\
\hline T84 & $6.13 \mathrm{E}-04$ \\
\hline SW620 & $8.76 \mathrm{E}-04$ \\
\hline SW837 & $7.36 \mathrm{E}-04$ \\
\hline COLO205 & $7.67 \mathrm{E}-04$ \\
\hline
\end{tabular}

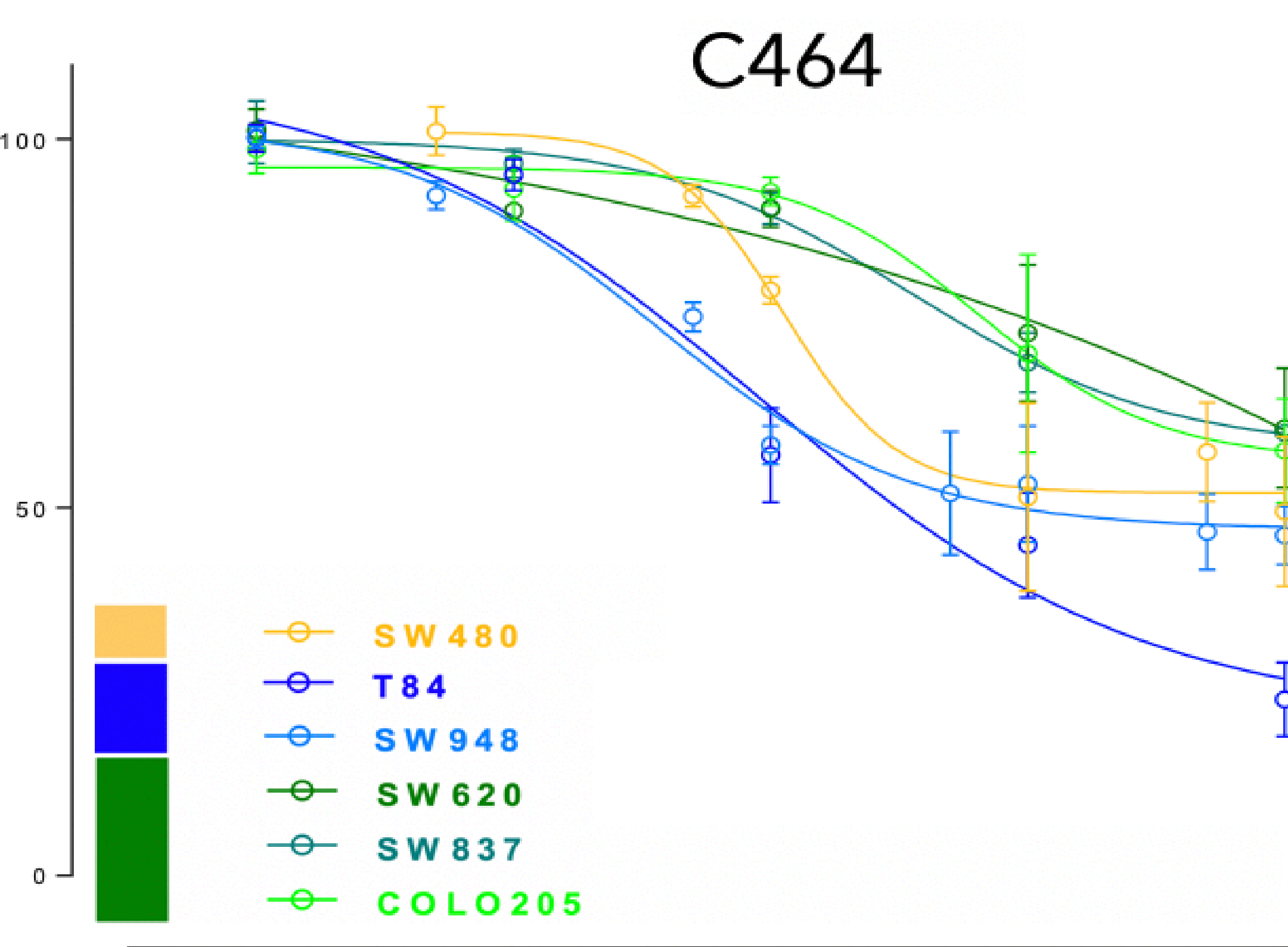

\begin{tabular}{|c|c|}
\hline & C464 \\
\hline SW480 & $5.53 E-04$ \\
\hline SW948 & $4.88 E-04$ \\
\hline T84 & $3.64 \mathrm{E}-04$ \\
SW620 & $6.88 \mathrm{E}-04$ \\
\hline SW837 & $6.66 \mathrm{E}-04$ \\
\hline COLO205 & $6.62 \mathrm{E}-04$ \\
\hline
\end{tabular}

[Inhibitor], M

[Inhibitor], M

B

AUC

\begin{tabular}{|c|c|c|c|c|c|c|c|c|c|c|}
\hline EpiC & CMS & & RVX208 & ISOX DUAL & MS436 & TP472 & BI-9564 & i-BET151 & (+)JO1 & C464 \\
\hline 1 & 1 & SW480 & 4 & 4.47E-04 & $7.11 \mathrm{E}-04$ & 6.64E-04 & 7.38E-04 & 3.61E-04 & 4.82E-04 & $=-04$ \\
\hline \multirow{2}{*}{$3 / 4$} & & 8 & 4 & 04 & 04 & 3.29E-04 & E-04 & -04 & & I \\
\hline & & T84 & 7.5 & $1.02 \mathrm{E}-04$ & 04 & $6.13 \mathrm{E}-04$ & 7.39E-04 & -04 & 3.4 & 3.64 \\
\hline \multirow{3}{*}{2} & \multirow{3}{*}{4} & & 04 & & & 8. & & -04 & 3 & $6.88 \mathrm{E}-04$ \\
\hline & & SW & $4.55 \mathrm{E}-04$ & $4.62 \mathrm{E}-04$ & 5.29E-04 & 7.36E-04 & 6.90E-04 & 5.09E-04 & 5.87E-04 & 6.66E-04 \\
\hline & & COLO205 & 1.87E-04 & 5.50E-04 & $8.36 \mathrm{E}-04$ & 7.67E-04 & 7.70E-04 & 7.63E-04 & 2.12E-03 & $6.62 \mathrm{E}-04$ \\
\hline
\end{tabular}

IC50

\begin{tabular}{|c|c|c|c|c|c|c|c|c|c|c|}
\hline EpiC & CMS & & RVX208 & ISOX DUAL & MS436 & TP472 & BI-9564 & i-BET151 & $(+) J Q 1$ & C464 \\
\hline 1 & 1 & SW480 & 6.55 & 7.03 & 6.54 & 6.35 & NA & 6.85 & 7.08 & 6.95 \\
\hline \multirow{2}{*}{$3 / 4$} & \multirow{2}{*}{2} & SW948 & 6.91 & 5.65 & 7.18 & 6.90 & 7.25 & 6.76 & 7.30 & 7.44 \\
\hline & & T84 & 7.26 & 7.14 & 6.98 & 6.86 & 6.83 & 6.08 & 6.98 & 7.04 \\
\hline \multirow{3}{*}{2} & \multirow{3}{*}{4} & SW620 & 6.60 & 6.55 & NA & NA & 7.03 & 6.36 & 5.23 & 6.19 \\
\hline & & SW837 & 5.65 & 6.28 & NA & NA & NA & NA & 4.91 & NA \\
\hline & & COLO205 & 6.30 & 5.55 & NA & NA & 4.87 & NA & NA & 6.44 \\
\hline
\end{tabular}



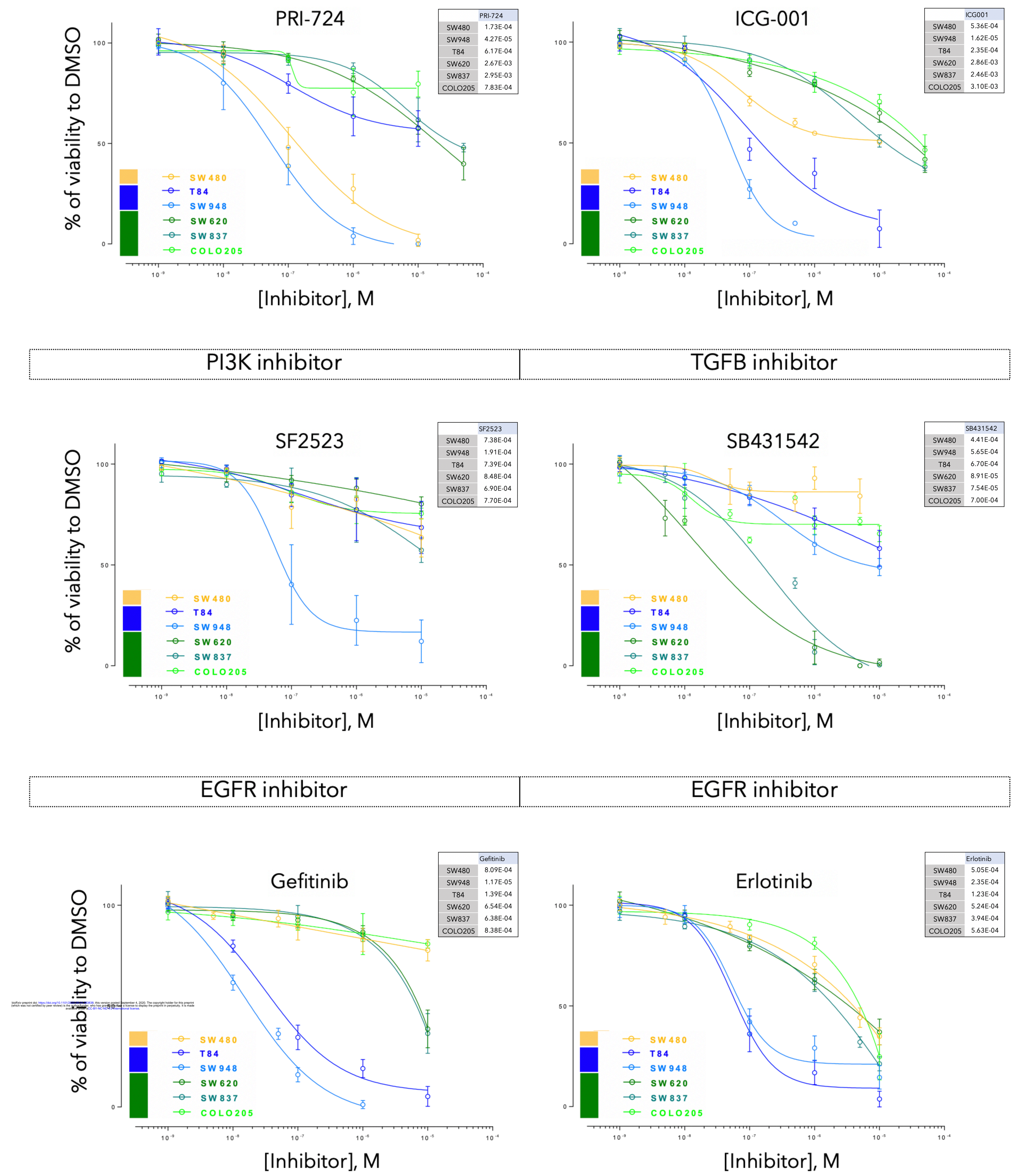


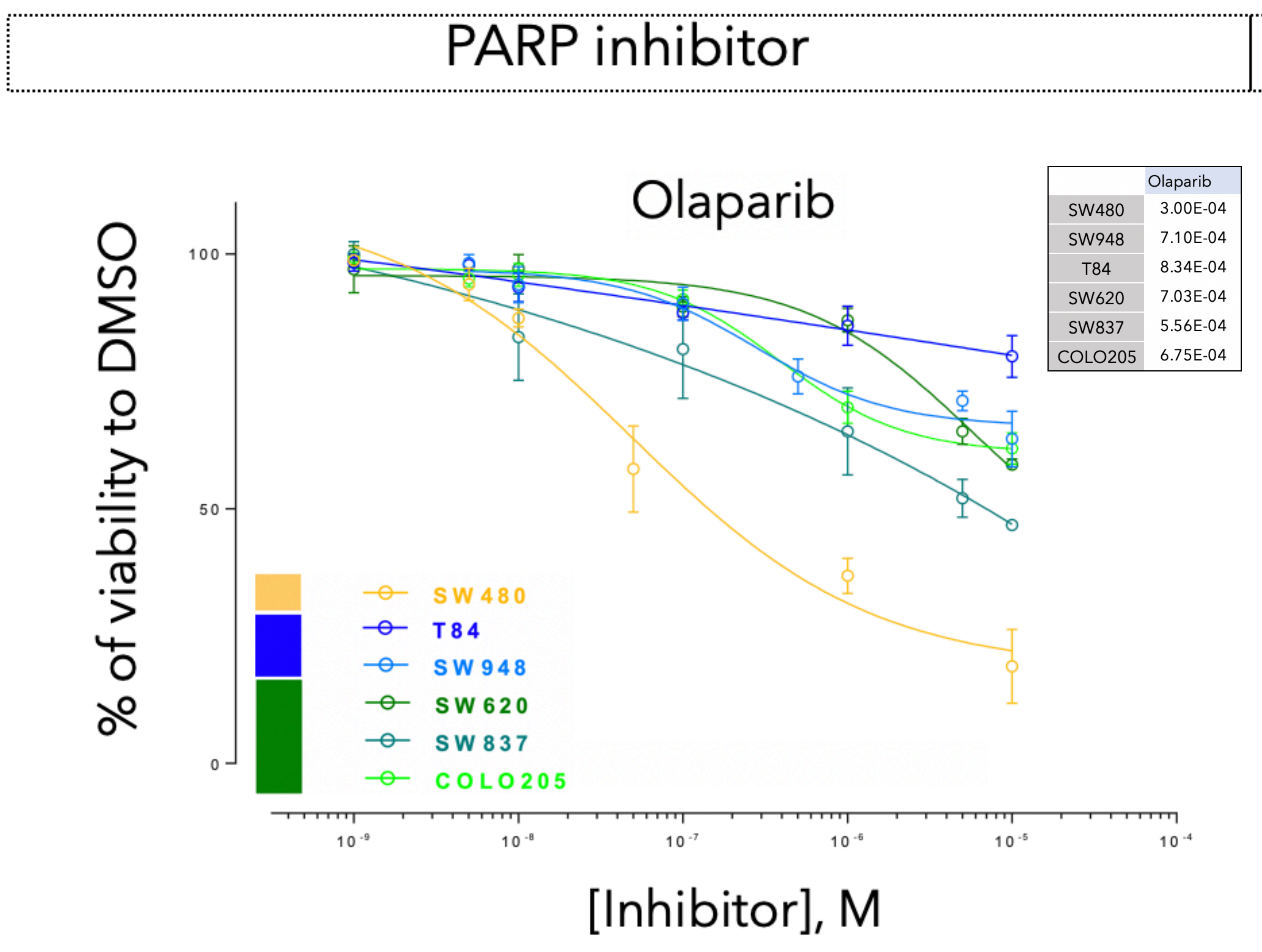

HER2 inhibitor

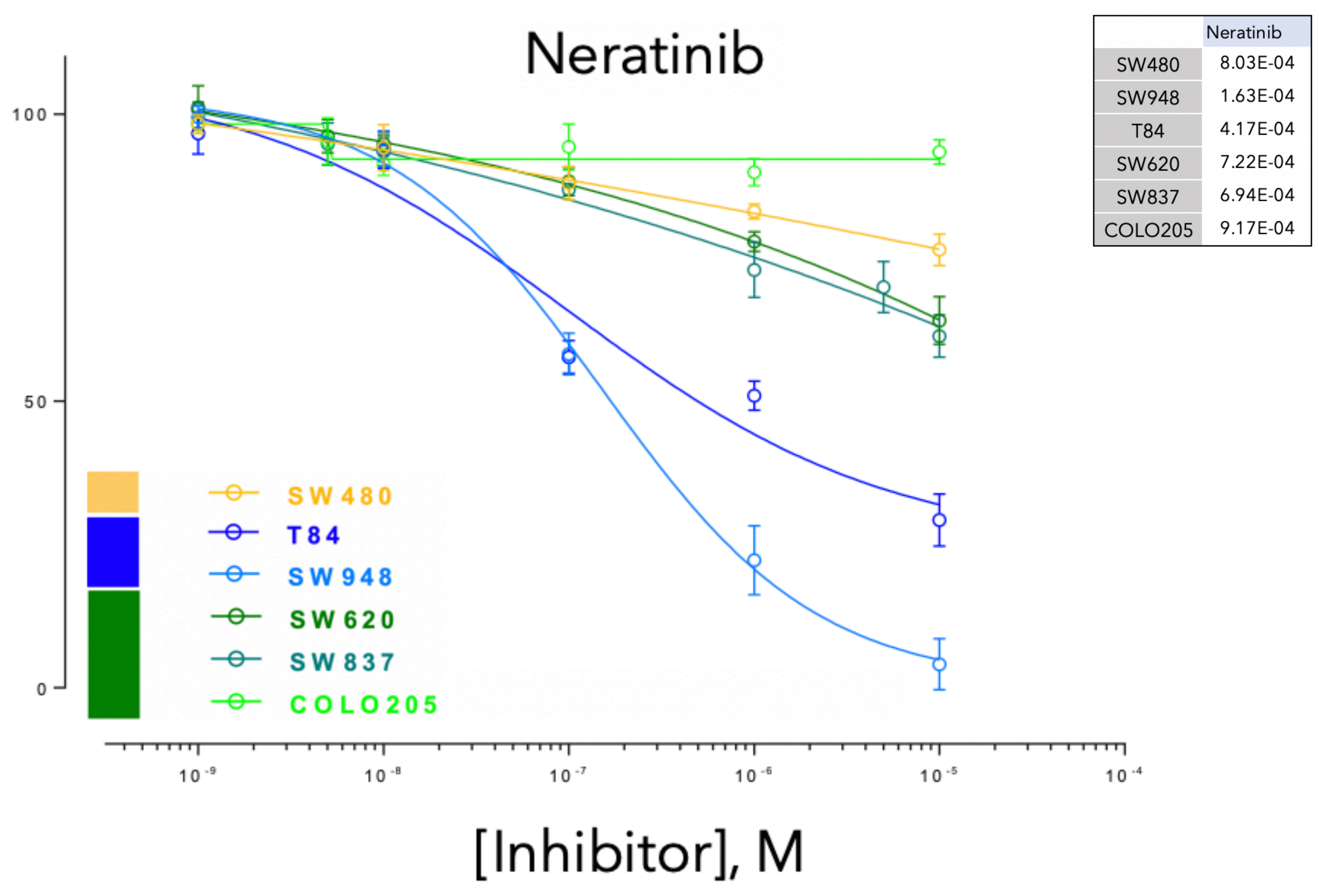

HSP90 inhibitor

HER2 inhibitor
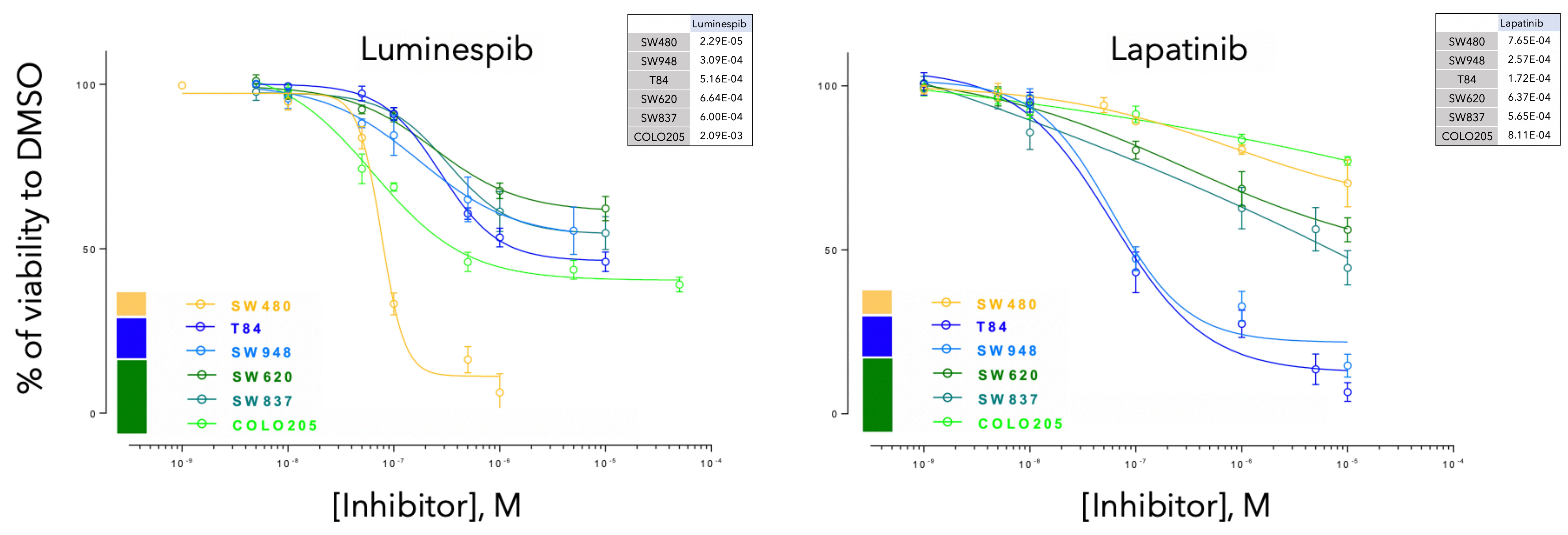

\section{PI3K inhibitor}

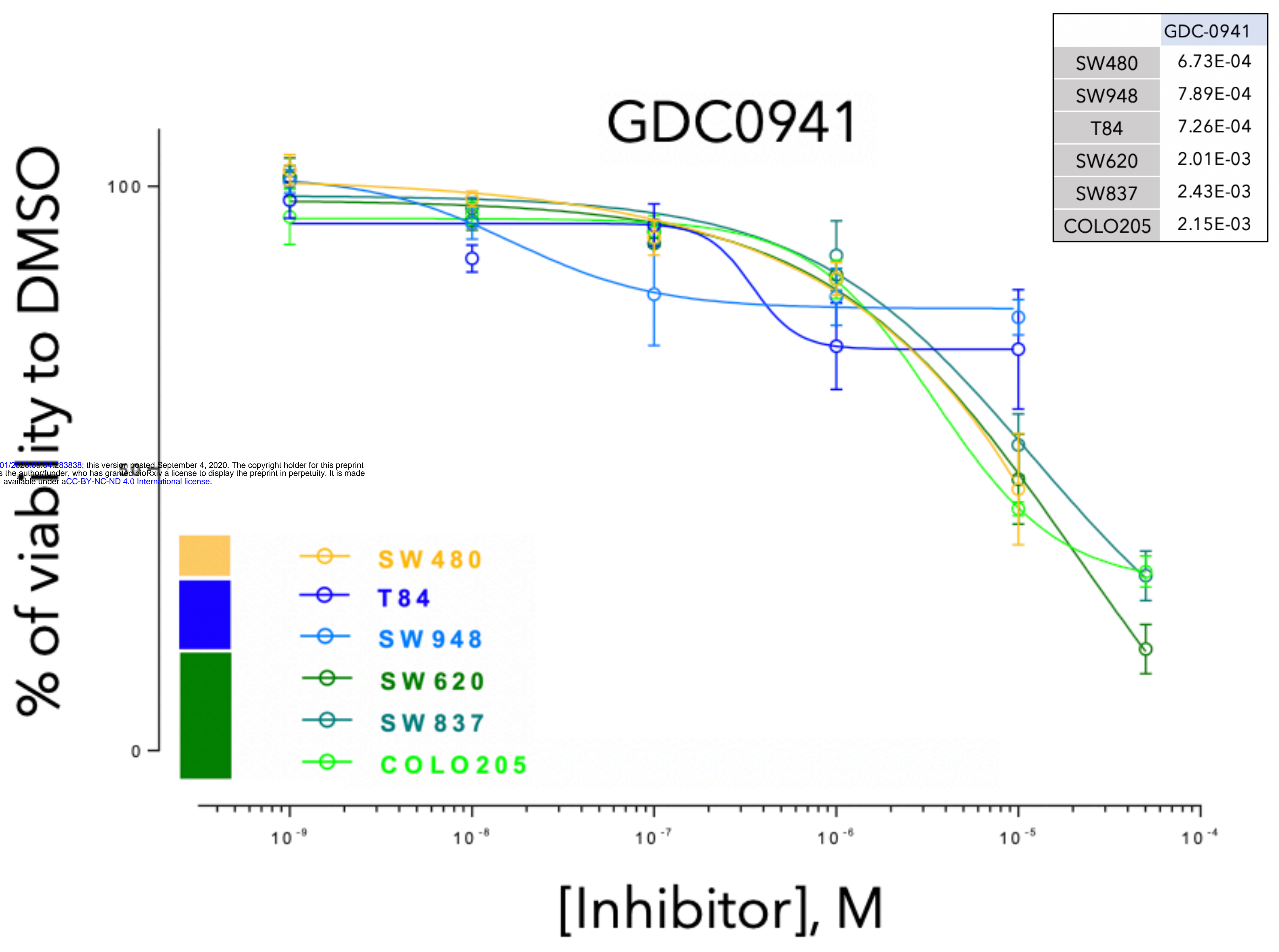




\section{Figure $\mathbf{S 1 3}$}

A

\begin{tabular}{|c|c|c|c|c|c|c|c|c|c|c|c|c|c|}
\hline EpiC & CMS & & GDC-0941 & SF2523 & ICG001 & PRI-724 & SB431542 & Erlotinib & Gefitinib & Neratinib & Lapatinib & Luminespib & Olaparib \\
\hline 1 & 1 & SW480 & $6.73 \mathrm{E}-04$ & $7.38 \mathrm{E}-04$ & $5.36 \mathrm{E}-04$ & 1.73E-04 & $4.41 \mathrm{E}-04$ & $5.05 \mathrm{E}-04$ & 8.09E-04 & $8.03 E-04$ & $7.65 \mathrm{E}-04$ & 2.29E-05 & $3.00 \mathrm{E}-04$ \\
\hline \multirow{2}{*}{$3 / 4$} & \multirow{2}{*}{2} & SW948 & 7.89E-04 & $1.91 \mathrm{E}-04$ & $1.62 \mathrm{E}-05$ & 4.27E-05 & $5.65 \mathrm{E}-04$ & $2.35 \mathrm{E}-04$ & 1.17E-05 & $1.63 \mathrm{E}-04$ & $2.57 \mathrm{E}-04$ & 3.09E-04 & $7.10 \mathrm{E}-04$ \\
\hline & & T84 & $7.26 \mathrm{E}-04$ & 7.39E-04 & $2.35 \mathrm{E}-04$ & 6.17E-04 & $6.70 \mathrm{E}-04$ & 1.23E-04 & $1.39 \mathrm{E}-04$ & 4.17E-04 & $1.72 \mathrm{E}-04$ & $5.16 \mathrm{E}-04$ & $8.34 \mathrm{E}-04$ \\
\hline \multirow{3}{*}{2} & \multirow{3}{*}{4} & SW620 & $2.01 \mathrm{E}-03$ & $8.48 \mathrm{E}-04$ & $2.86 \mathrm{E}-03$ & 2.67E-03 & 8.91E-05 & $5.24 \mathrm{E}-04$ & $6.54 \mathrm{E}-04$ & $7.22 \mathrm{E}-04$ & $6.37 \mathrm{E}-04$ & $6.64 \mathrm{E}-04$ & 7.03E-04 \\
\hline & & SW837 & 2.43E-03 & $6.90 \mathrm{E}-04$ & $2.46 \mathrm{E}-03$ & $2.95 \mathrm{E}-03$ & 7.54E-05 & $3.94 \mathrm{E}-04$ & $6.38 \mathrm{E}-04$ & $6.94 \mathrm{E}-04$ & $5.65 \mathrm{E}-04$ & $6.00 \mathrm{E}-04$ & $5.56 \mathrm{E}-04$ \\
\hline & & COLO205 & $2.15 \mathrm{E}-03$ & 7.70E-04 & $3.10 \mathrm{E}-03$ & 7.83E-04 & 7.00E-04 & $5.63 \mathrm{E}-04$ & $8.38 \mathrm{E}-04$ & $9.17 \mathrm{E}-04$ & $8.11 \mathrm{E}-04$ & $2.09 \mathrm{E}-03$ & $6.75 \mathrm{E}-04$ \\
\hline
\end{tabular}

\begin{tabular}{|c|c|c|c|c|c|c|c|c|c|c|c|c|c|}
\hline EpiC & CMS & & GDC-0941 & SF2523 & ICG001 & PRI-724 & SB431542 & Erlotinib & Gefitinib & Neratinib & Lapatinib & Luminespib & Olaparib \\
\hline 1 & 1 & SW480 & NA & NA & 7.11 & 6.95 & 7.59 & NA & NA & NA & 6.09 & 7.11 & 7.31 \\
\hline \multirow{2}{*}{$3 / 4$} & \multirow{2}{*}{2} & SW948 & 7.77 & 7.25 & 7.31 & 7.25 & 6.49 & 7.27 & 7.82 & 6.81 & 7.22 & 6.75 & 6.55 \\
\hline & & T84 & 6.46 & 6.83 & 7.04 & 6.95 & NA & 7.26 & 7.50 & 6.99 & 7.22 & 6.56 & NA \\
\hline \multirow{3}{*}{2} & \multirow{3}{*}{4} & SW620 & 5.45 & 7.03 & NA & NA & 7.89 & NA & 5.21 & NA & NA & 7.19 & 6.41 \\
\hline & & SW837 & 4.68 & NA & NA & 4.64 & 7.85 & NA & NA & NA & 6.54 & 6.61 & 5.24 \\
\hline & & COLO205 & 4.92 & 4.87 & 5.38 & 5.20 & 6.71 & 4.56 & NA & NA & NA & 6.49 & NA \\
\hline
\end{tabular}

B
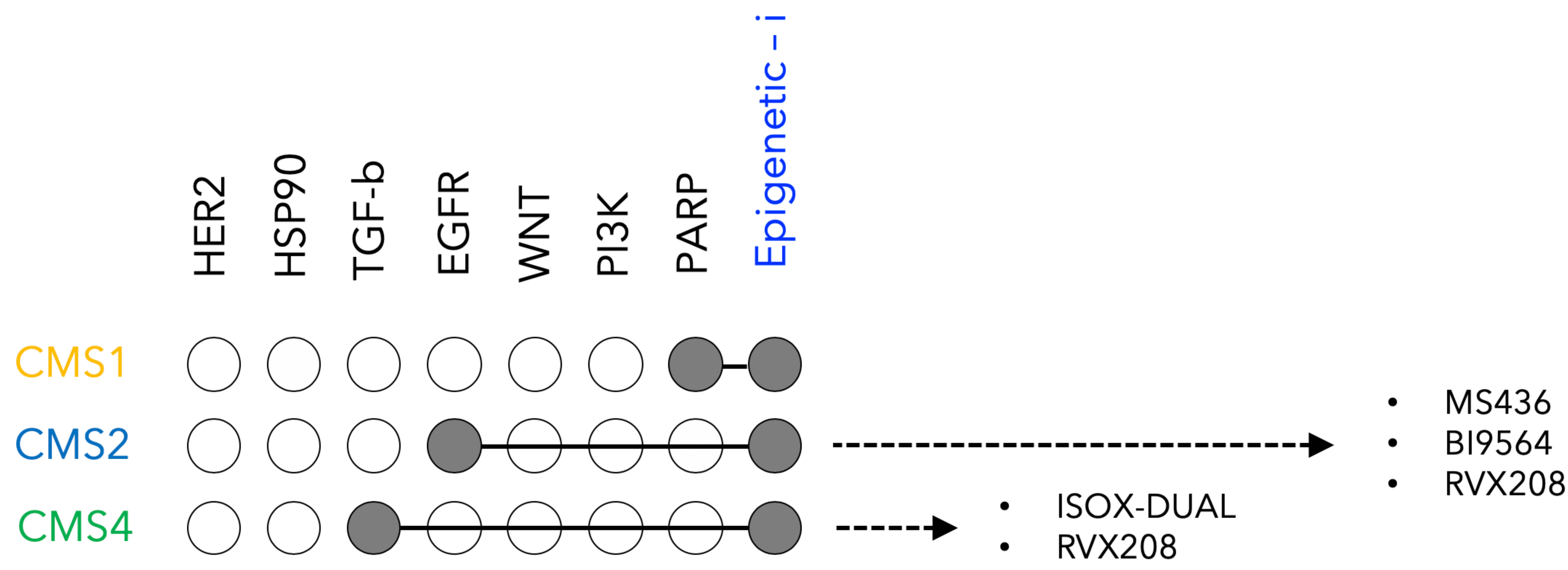
A

CMS1

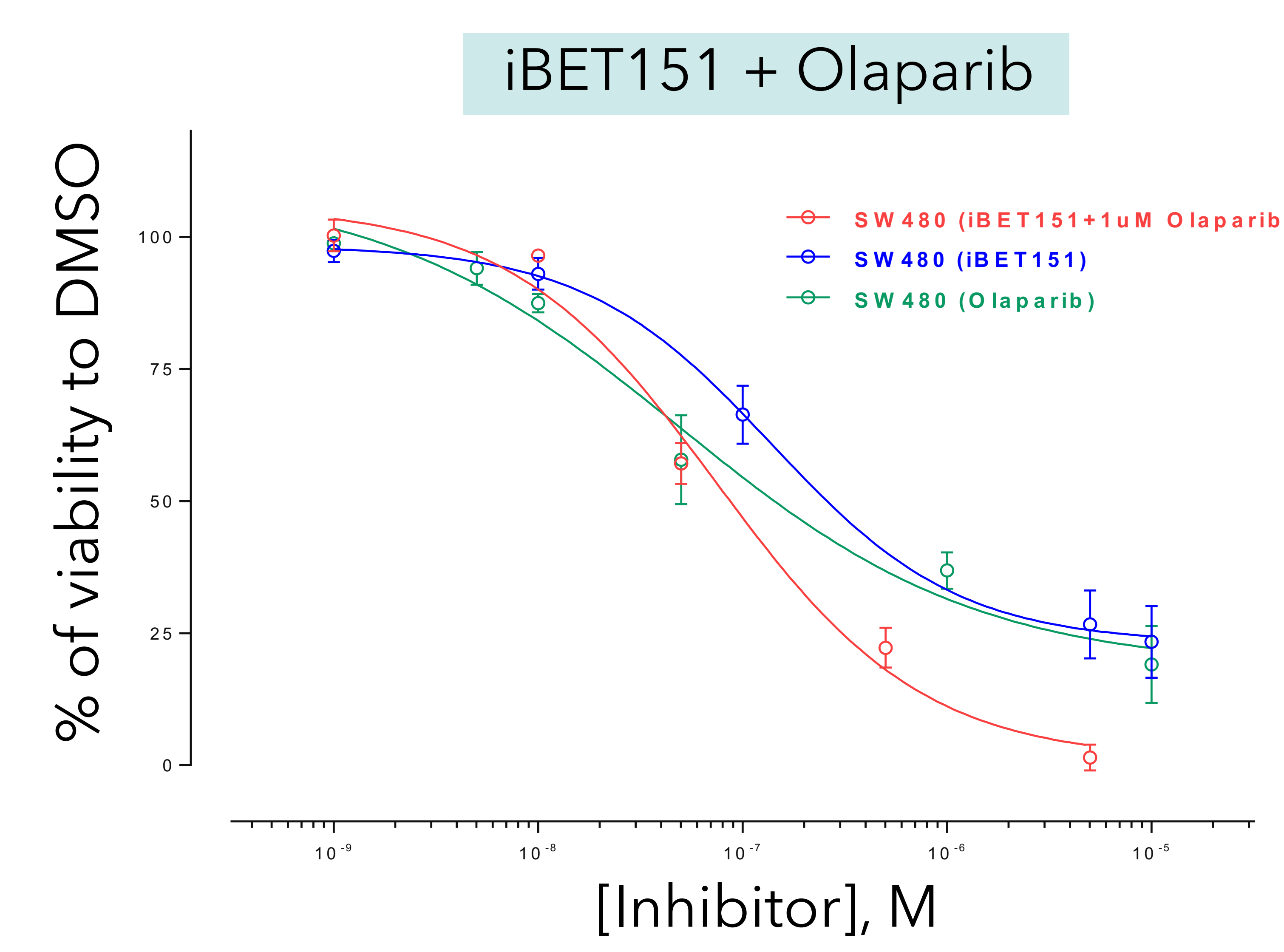

B

\section{EpiC1/CMS1}

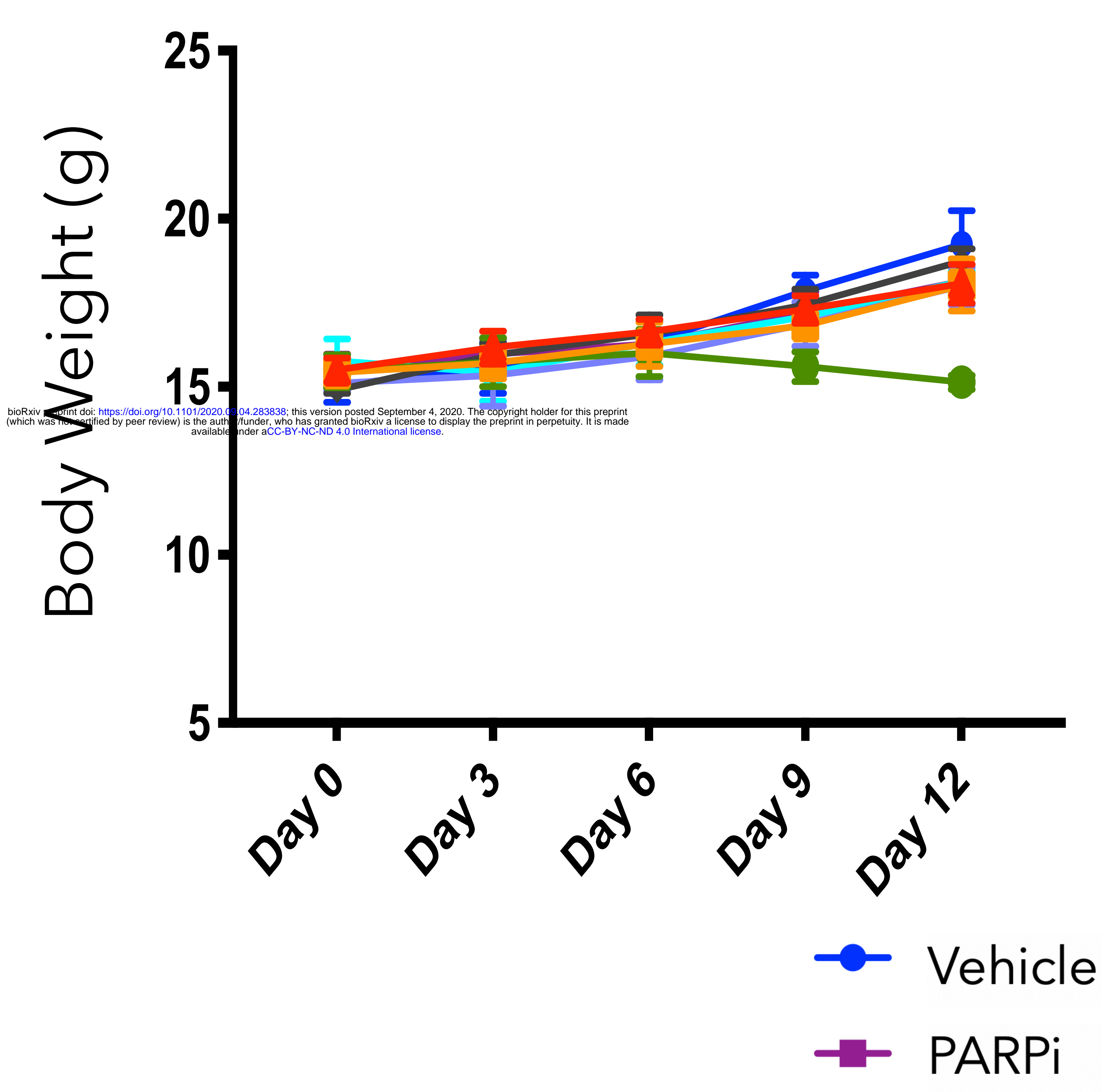

Combinatorial Treatments

\section{CMS2}

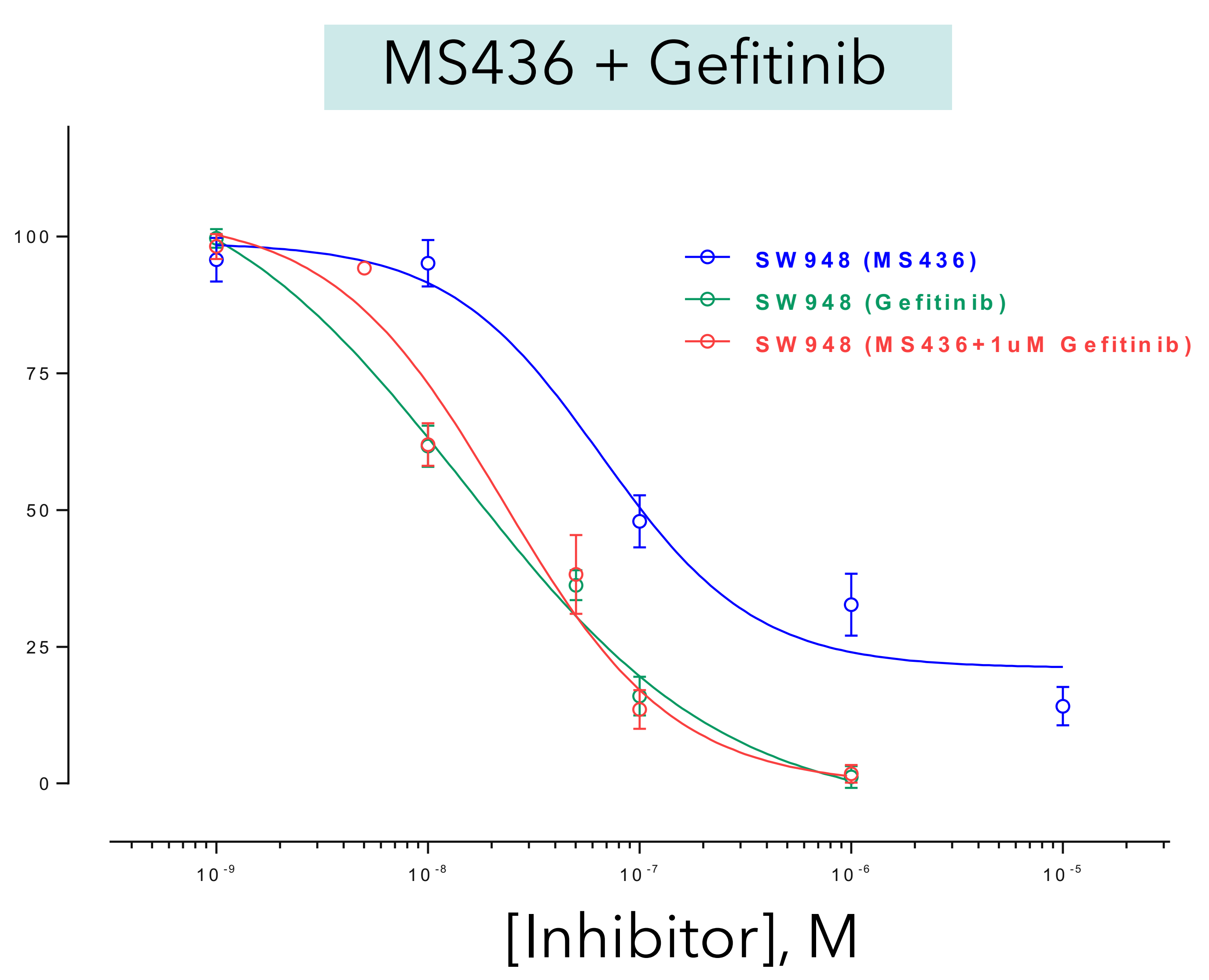

B19564 + Gefitinib

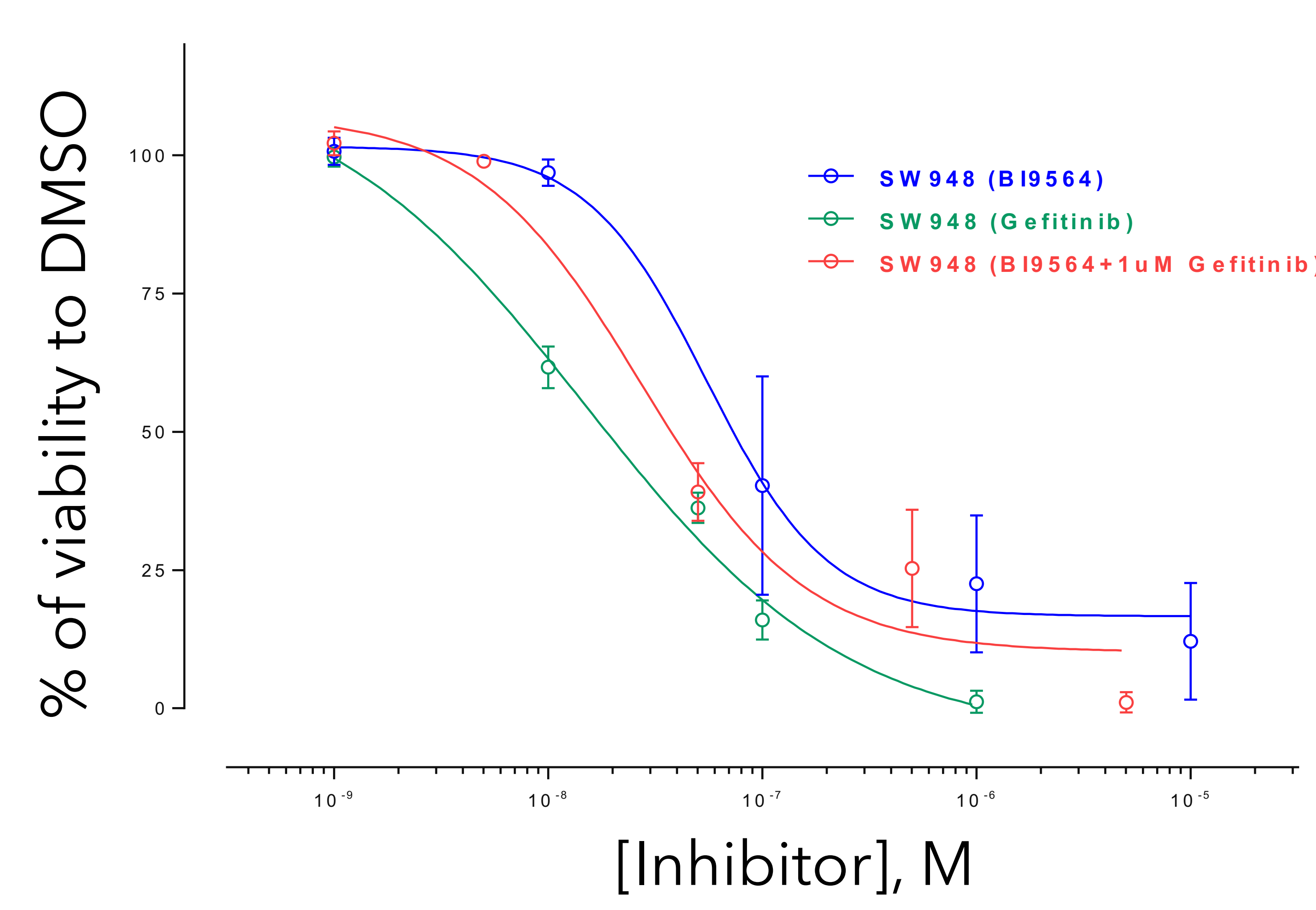

RVX208+ Gefitinib

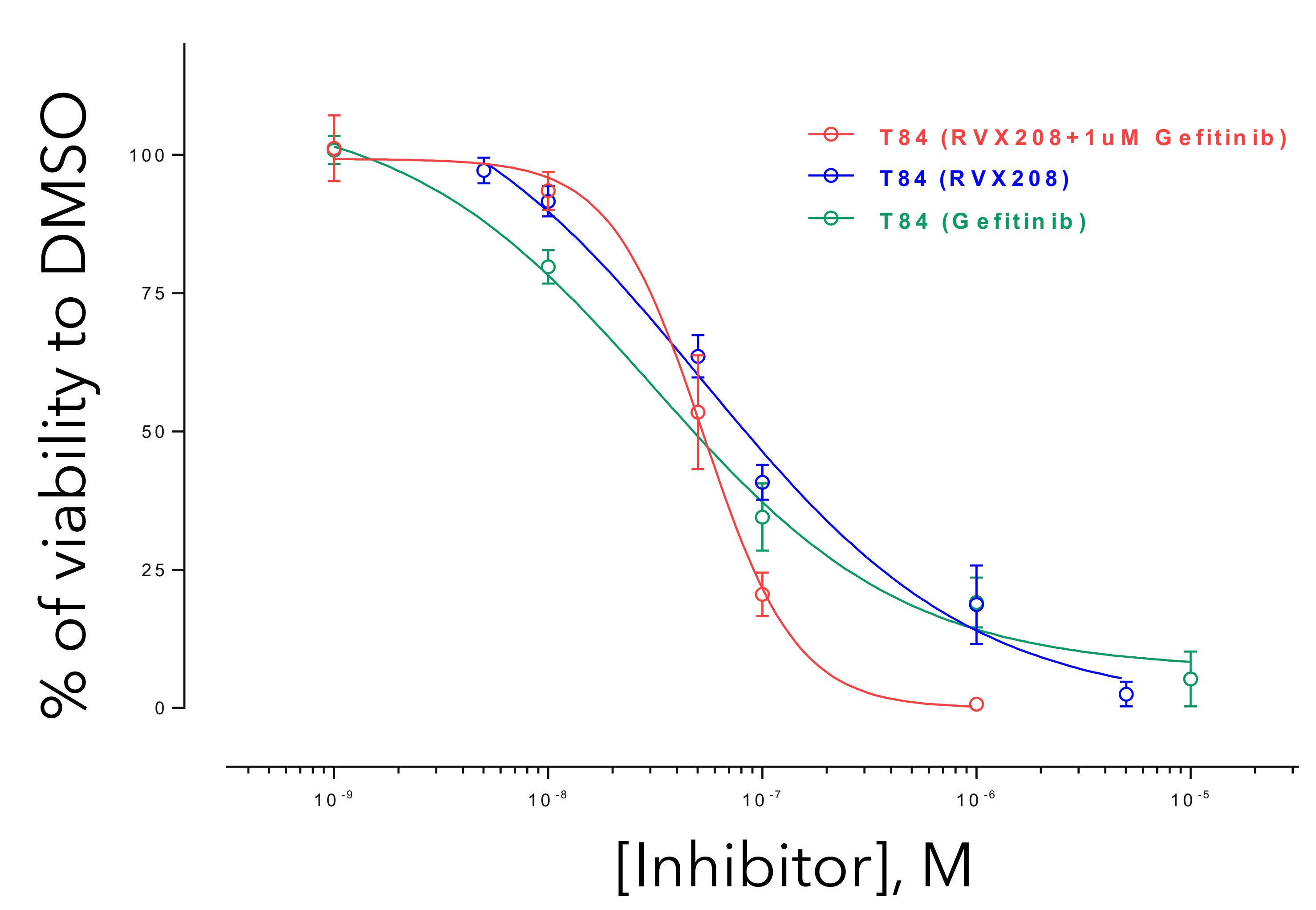

EpiC2/CMS4

\section{EpiC3-4/CMS2}

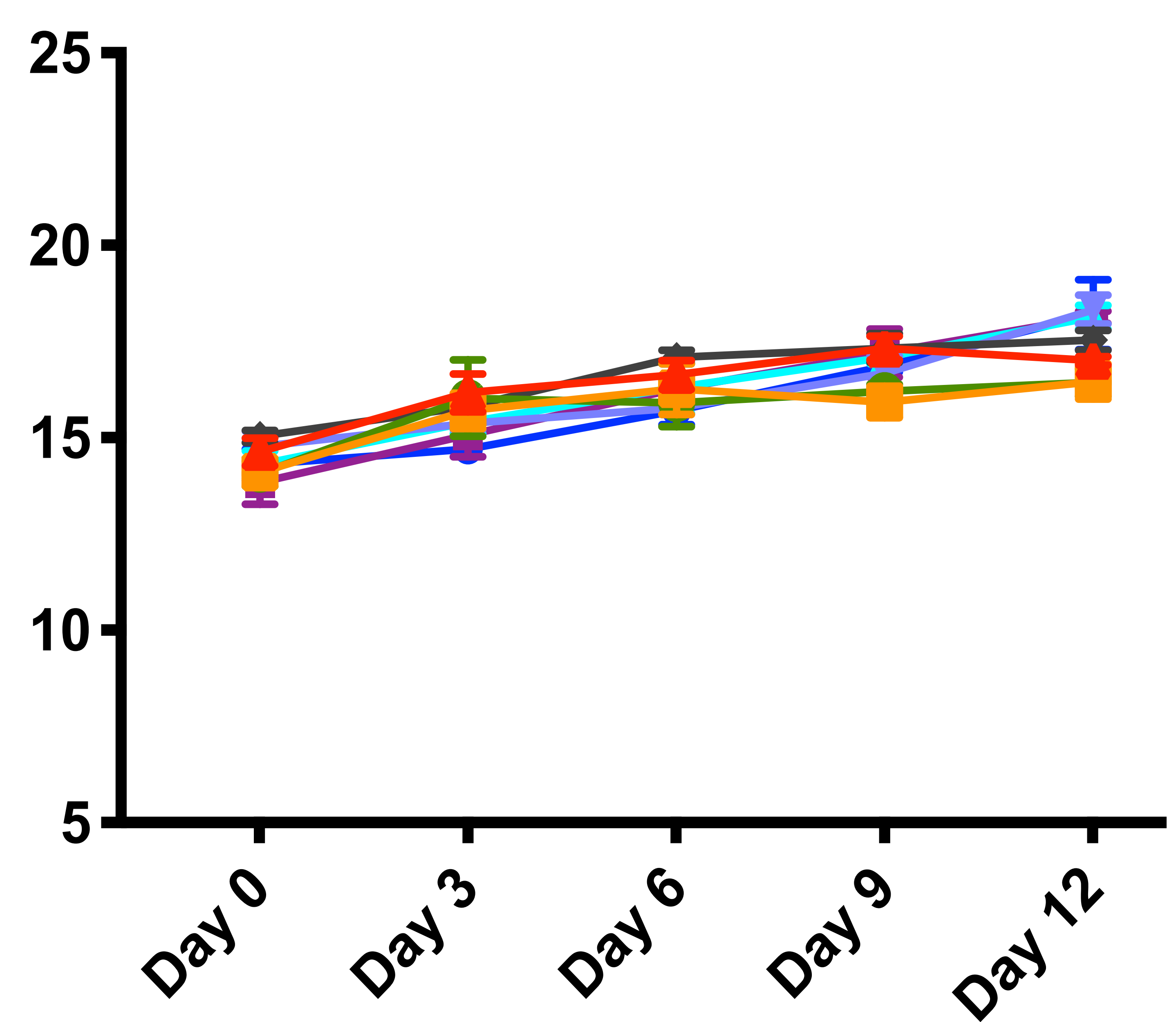

\section{CMS4}

ISO-DUAL + SB431542

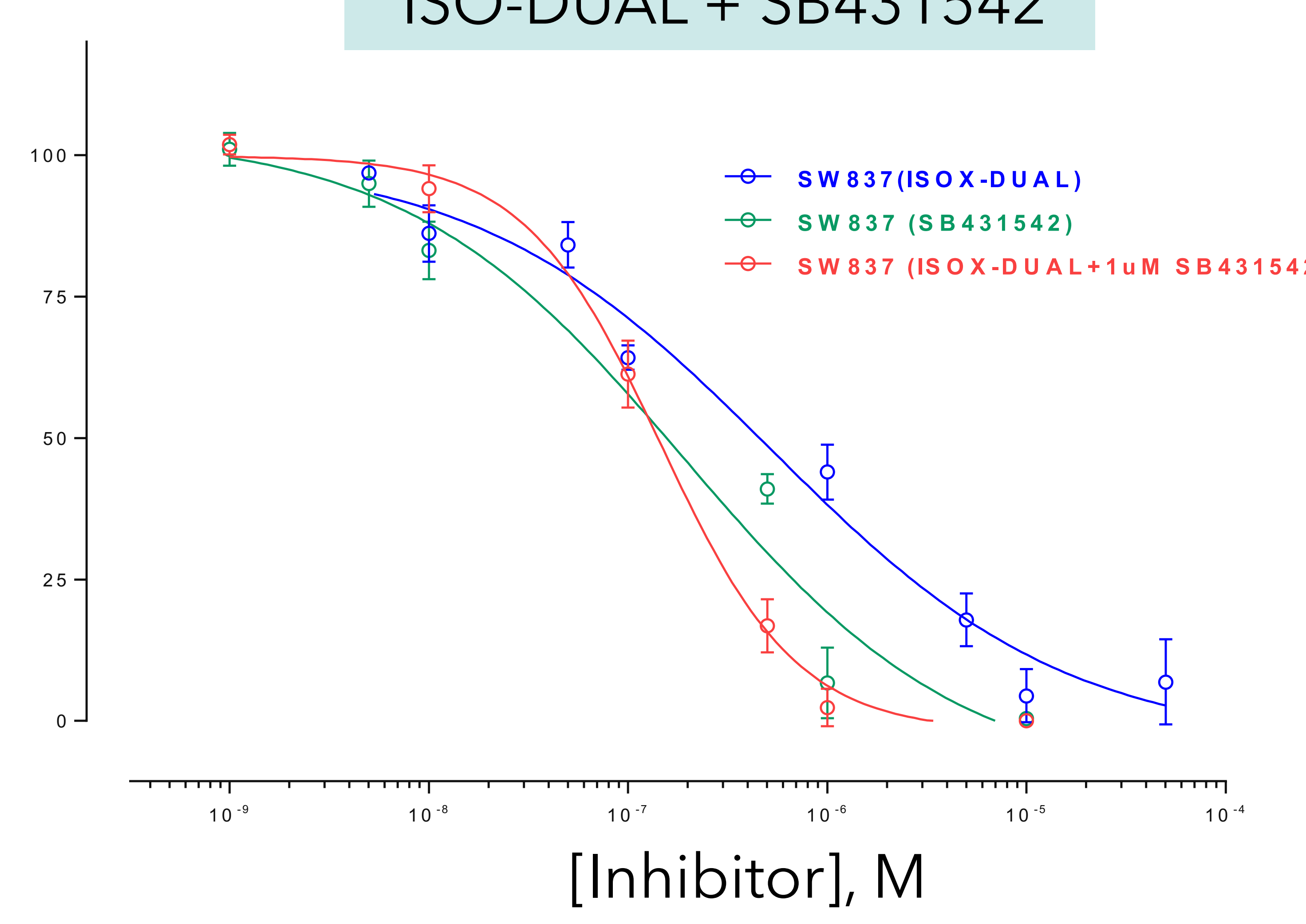

ISO-DUAL + SB431542

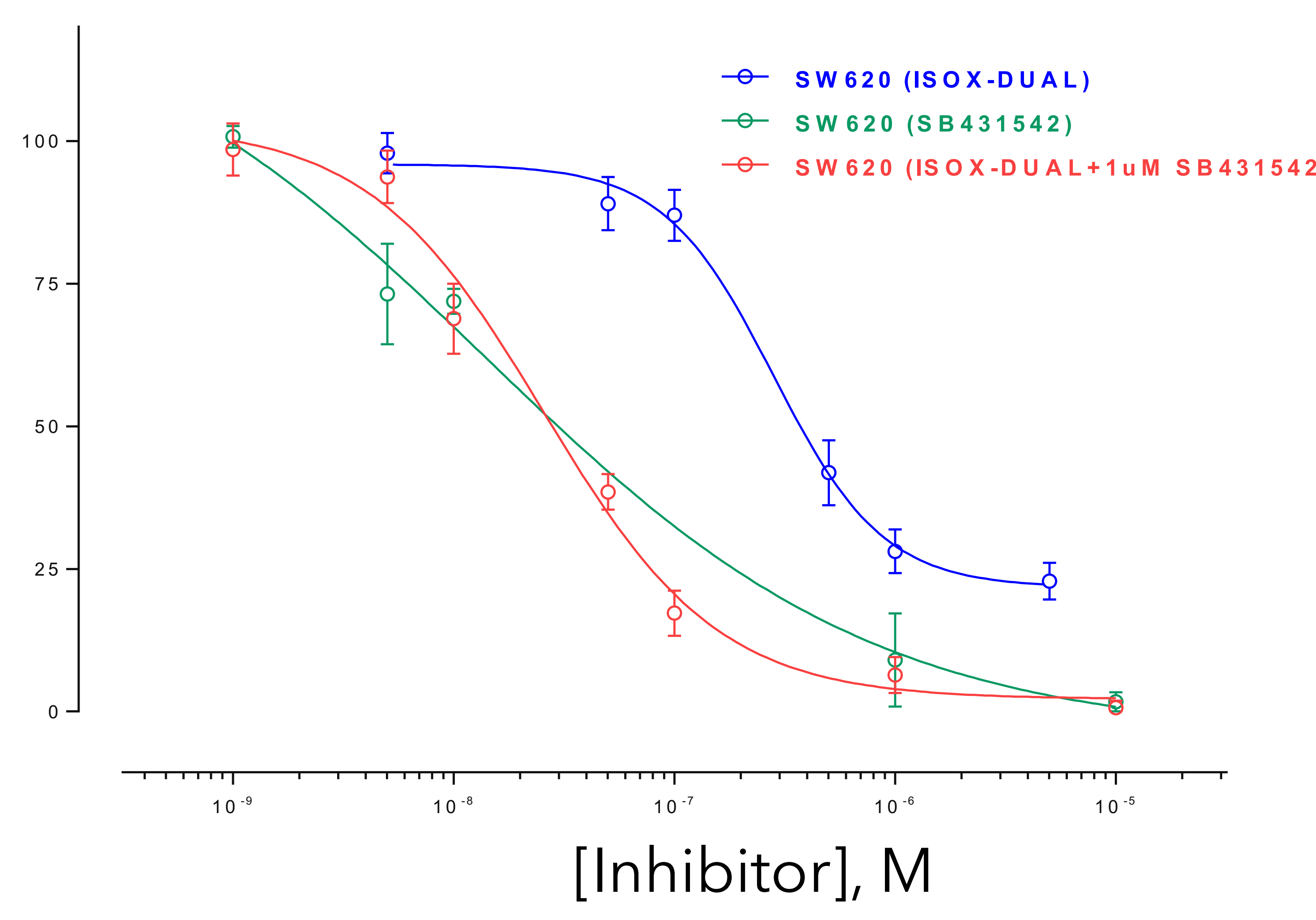

RVX208 + SB431542

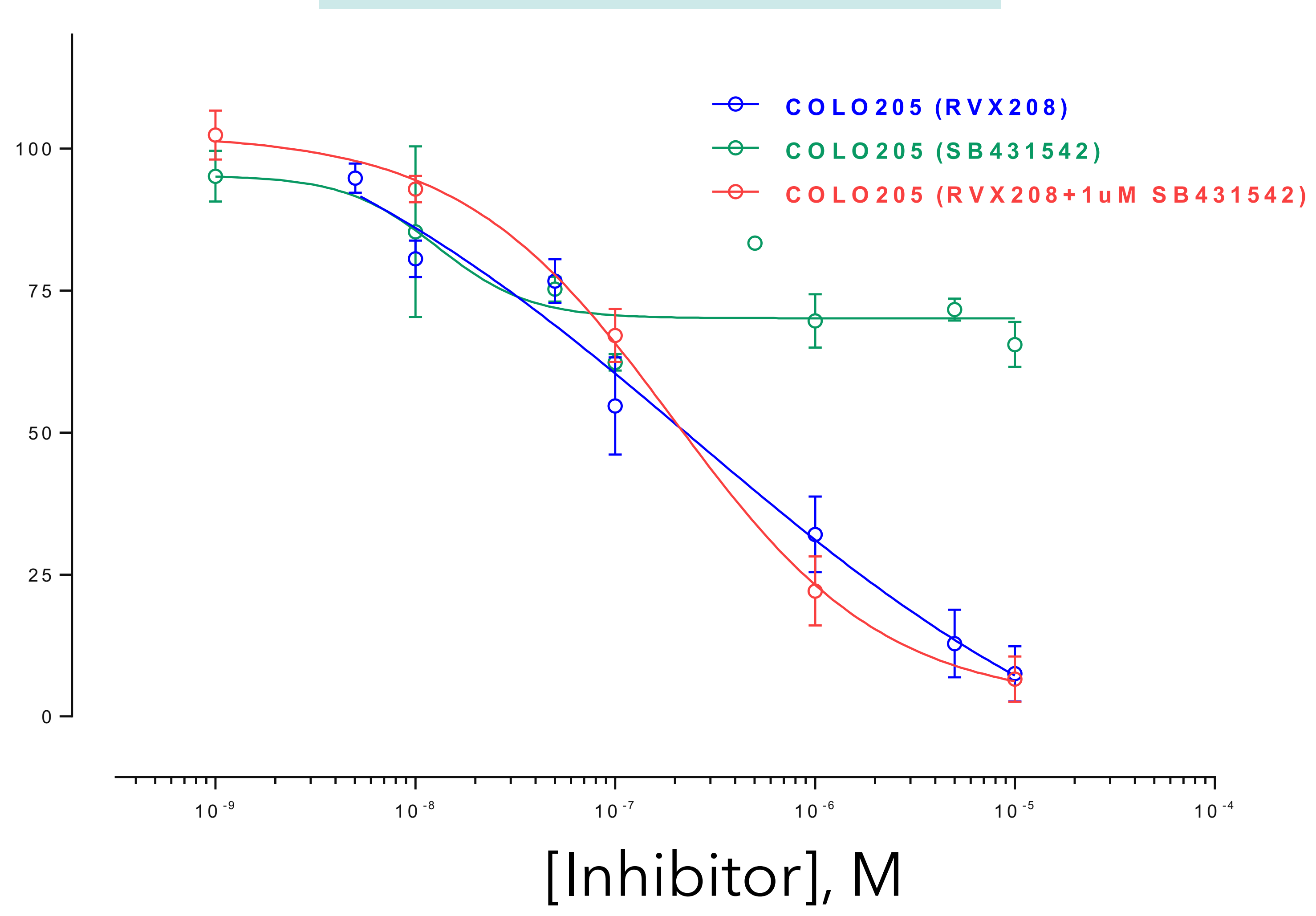

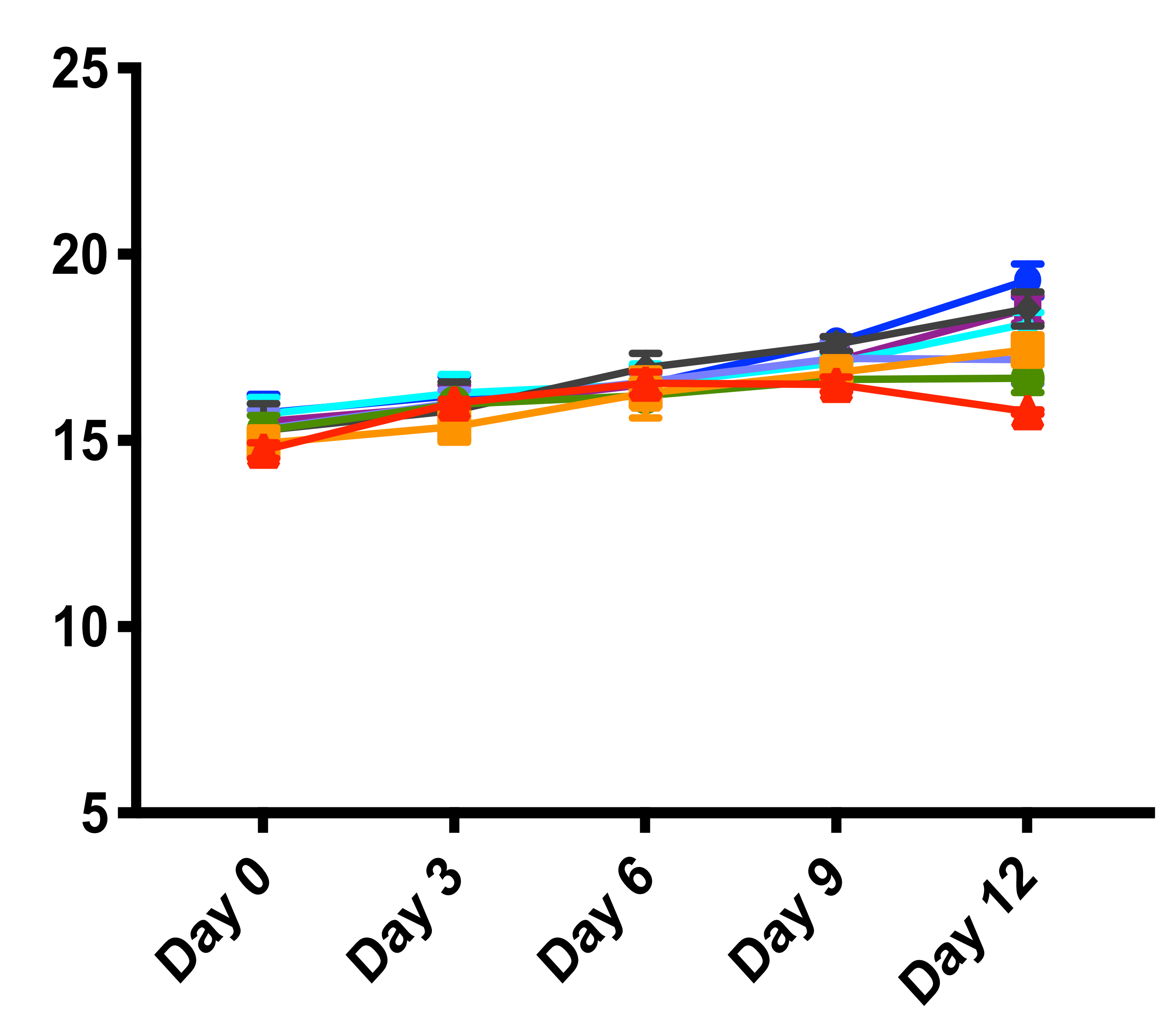

$$
\begin{array}{ll}
\leftarrow \text { EGFRi } & \rightarrow \text { BRDi } \\
\rightarrow \mathrm{TGFBi} & \rightarrow \mathrm{PARPi}+\mathrm{BRDi} \leftarrow \mathrm{EGFRi}+\mathrm{BRDi}
\end{array}
$$

